\title{
Building Industry Reporting and Design for Sustainability (BIRDS) Commercial Database Technical Manual: Update
}

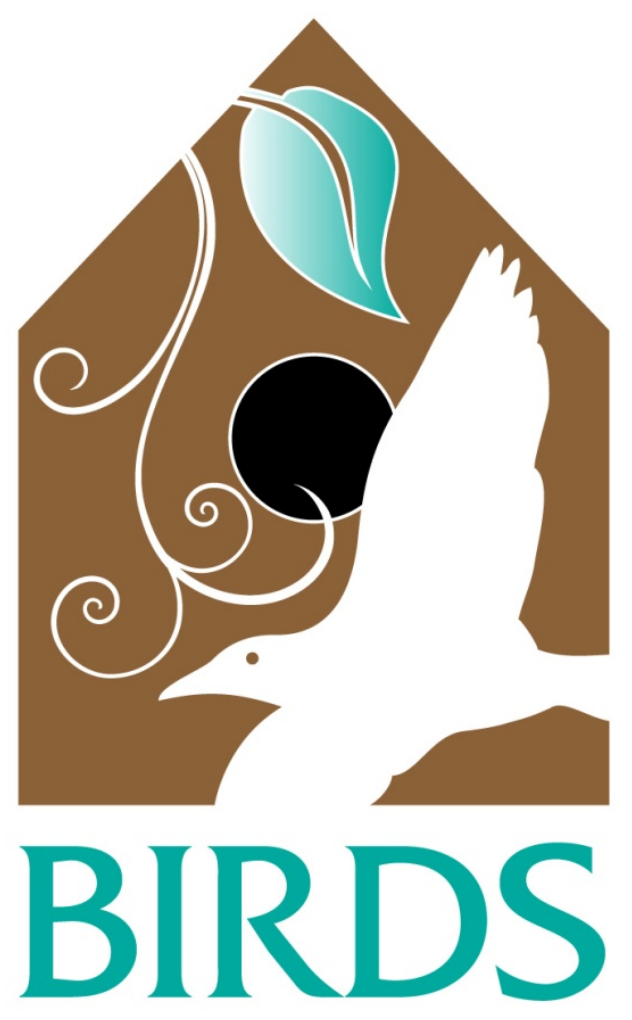

Joshua Kneifel Eric O’Rear Priya Lavappa Shannon Grubb Anne Landfield Greig Sangwon Suh

This publication is available free of charge from: https://doi.org/10.6028/NIST.TN.1975r2

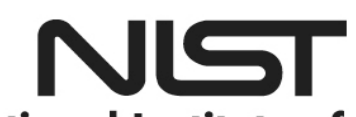

National Institute of Standards and Technology U.S. Department of Commerce 



\title{
Building Industry Reporting and Design for Sustainability (BIRDS) Commercial Database Technical Manual: Update
}

\author{
Joshua Kneifel \\ Eric O’Rear \\ Priya Lavappa \\ Shannon Grubb \\ Applied Economics Office \\ Engineering Laboratory \\ Anne Landfield Greig \\ Four Elements Consulting, LLC \\ Sangwon Suh \\ Industrial Ecology Research Services, LLC
}

This publication is available free of charge from:

https://doi.org/10.6028/NIST.TN.1975r2

September 2019

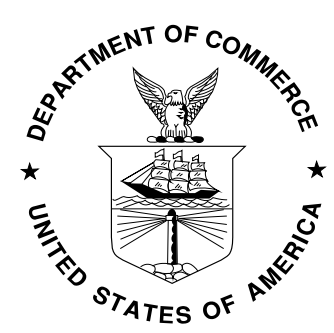

U.S. Department of Commerce Wilbur L. Ross, Jr., Secretary

National Institute of Standards and Technology Walter Copan, NIST Director and Under Secretary of Commerce for Standards and Technology 
Certain commercial entities, equipment, or materials may be identified in this document in order to describe an experimental procedure or concept adequately. Such identification is not intended to imply recommendation or endorsement by the National Institute of Standards and Technology, nor is it intended to imply that the entities, materials, or equipment are necessarily the best available for the purpose.

National Institute of Standards and Technology Technical Note 1975r2 Natl. Inst. Stand. Technol. Tech. Note 1975r2, 152 pages (September 2019) CODEN: NTNOEF

This publication is available free of charge from: https://doi.org/10.6028/NIST.TN.1975r2 


\begin{abstract}
Building stakeholders need practical metrics, data, and tools to support decisions related to sustainable building designs, technologies, standards, and codes. The Engineering Laboratory of the National Institute of Standards and Technology (NIST) has addressed this high priority national need by extending its metrics and tools for sustainable building products, known as Building for Environmental and Economic Sustainability (BEES), to whole-buildings. Wholebuilding sustainability metrics have been developed based on innovative extensions to life-cycle assessment (LCA) and life-cycle costing (LCC) approaches involving whole-building energy simulations. The measurement system evaluates the sustainability of both the materials and the energy used by a building over time. It assesses the "carbon footprint" of buildings as well as 11 other environmental performance metrics and integrates economic performance metrics to yield science-based measures of the business case for investment choices in high-performance, green buildings.

Building Industry Reporting and Design for Sustainability (BIRDS) applies the sustainability measurement system to an extensive whole-building performance database NIST has compiled for this purpose. In addition to the database developed for previous versions of BIRDS, the updated BIRDS Commercial database now includes energy, environmental, and cost measurements for 17100 new commercial and non-low rise residential buildings. These buildings cover the 15 building prototypes based on the PNNL Commercial Prototype Building Models in 228 cities across all U.S. states for study period lengths ranging from 1 year to 40 years. The sustainability performance of buildings designed to meet current state energy codes can be compared to their performance when meeting four alternative building energy standard editions to determine the impact of energy efficiency on sustainability performance. The impact of the building location and the investor's time horizon on sustainability performance can also be measured.
\end{abstract}

\title{
Keywords
}

Building economics; economic analysis; life-cycle costing; life-cycle assessment; energy efficiency; commercial buildings. 


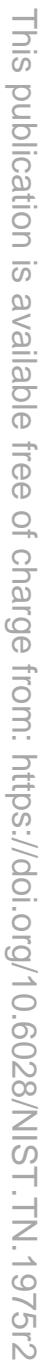




\section{Preface}

This documentation was developed by the Applied Economics Office (AEO) in the Engineering Laboratory (EL) at the National Institute of Standards and Technology (NIST). The document explains how the BIRDS commercial database was updated, including the assumptions and data sources for the energy, environmental, and cost estimate calculations. The intended audience is BIRDS v4.0 users, researchers and decision makers in the commercial building sector, and others interested in building sustainability.

\section{Disclaimers}

The policy of the National Institute of Standards and Technology is to use metric units in all its published materials. Because this report is intended for the U.S. construction industry which uses U.S. customary units, it is more practical and less confusing to include U.S. customary units as well as metric units. Measurement values in this report are therefore stated in metric units first, followed by the corresponding values in U.S. customary units within parentheses.

Certain commercial entities, equipment, or materials may be identified in this document to describe an experimental procedure or concept adequately. Such identification is not intended to imply recommendation or endorsement by the National Institute of Standards and Technology, nor is it intended to imply that the entities, materials, or equipment are necessarily the best available for the purpose. 


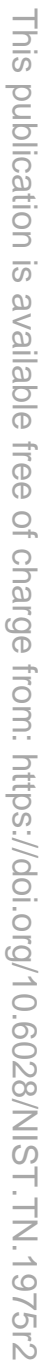




\section{Acknowledgements}

The authors wish to thank all those who contributed ideas and suggestions for this report. They include Dr. Cheyney O’Fallon and Dr. David Butry of EL’s Applied Economics Office, Mr. William S. Dols and Brian Dougherty of EL's Energy and Environment Division, and Dr. Nicos S. Martys of EL's Materials and Structural Systems Division. A special thanks to the Industrial Ecology Research Services team of Ms. Shivira Tomar, Ms. Christine Chen, and Mr. Matthew Leighton for their superb technical support in developing whole-building environmental life-cycle assessments for BIRDS. Thanks goes to our industry contacts that were instrumental in advising on the assumptions used to develop the product-level life-cycle impact assessments. Finally, the many Beta testers of BIRDS deserve special thanks for contributing suggestions leading to substantial improvements in the tool.

\section{Author Information}

Joshua Kneifel

Economist

National Institute of Standards and Technology

Engineering Laboratory

100 Bureau Drive, Mailstop 8603

Gaithersburg, MD 208998603

Tel.: 301-975-6857

Email: joshua.kneifel@nist.gov

Eric O’Rear

Economist

National Institute of Standards and Technology

Engineering Laboratory

100 Bureau Drive, Mailstop 8603

Gaithersburg, MD 208998603

Tel.: 301-975-4570

Email: eric.orear@nist.gov

Priya Lavappa

Computer Specialist

National Institute of Standards and Technology

Engineering Laboratory

100 Bureau Drive, Mailstop 8603

Gaithersburg, MD 208998603

Tel.: 301-975-4522

Email: priya.lavappa@nist.gov 
Shannon Grubb

Computer Specialist

National Institute of Standards and Technology

Engineering Laboratory

100 Bureau Drive, Mailstop 8603

Gaithersburg, MD 208998603

Tel.: 301-975-6857

Email: shannon.grubb@nist.gov

Anne Landfield Greig

Principal

Four Elements Consulting, LLC

Seattle, WA

Tel: 206-935-4600

Email: anne@fourelementsllc.com

Sangwon Suh

Director and Founder

Industrial Ecology Research Services (IERS), LLC

5951 Encina Rd, Suite 206

Goleta, CA 93117

Tel: 805-324-4674

Email: suh@iersweb.com 


\section{Contents}

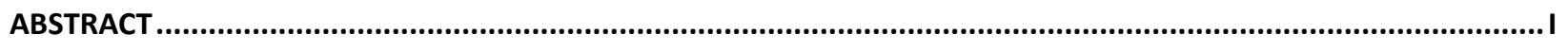

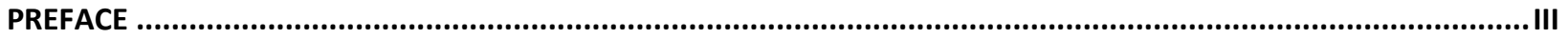

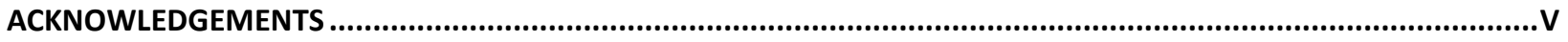

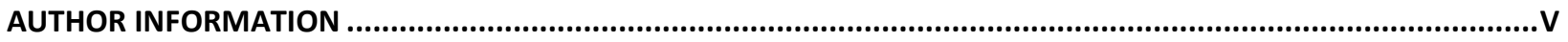

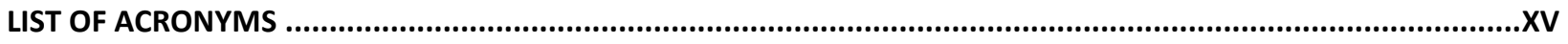

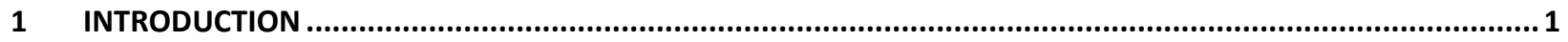

1.1 PURPOSE

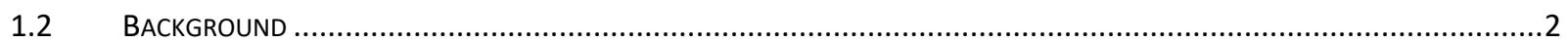

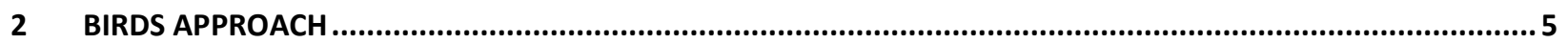

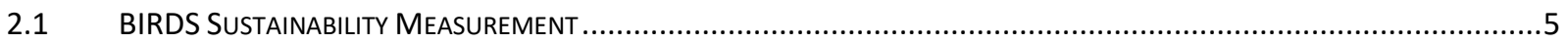

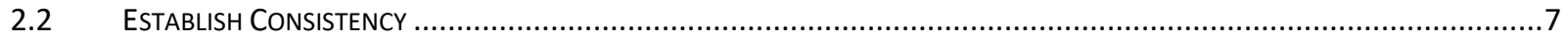

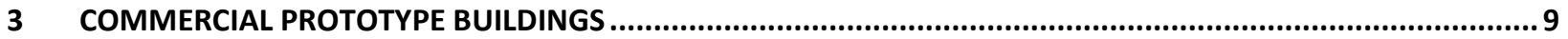

$3.1 \quad$ BUILDING FORM

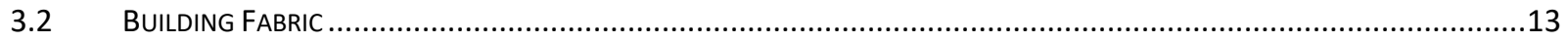

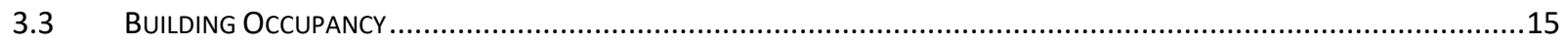

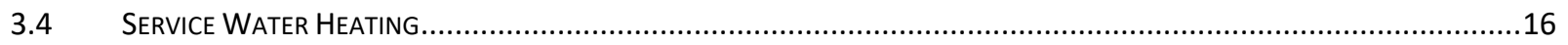

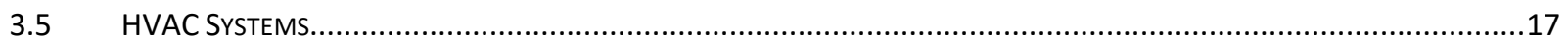

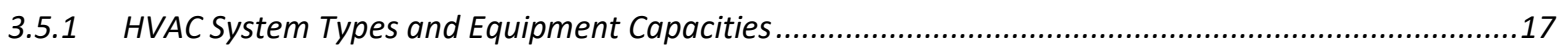

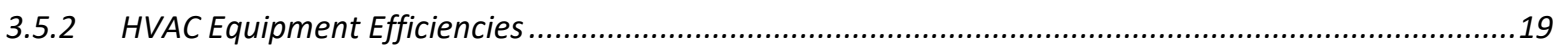

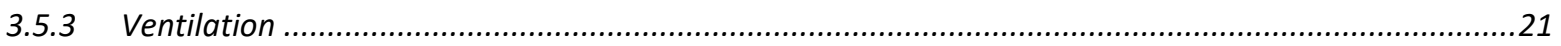

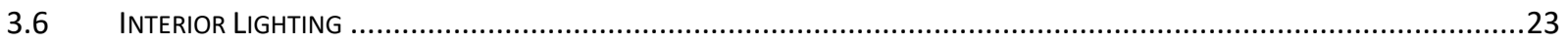

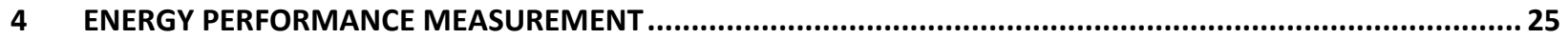

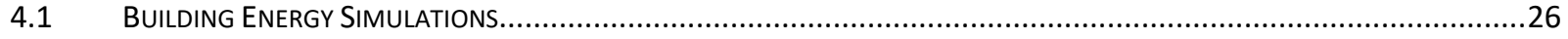

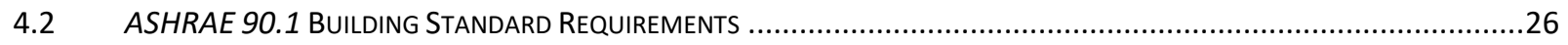

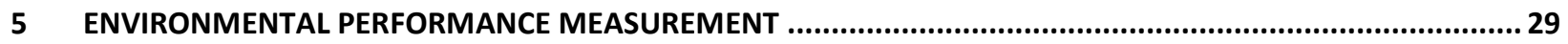

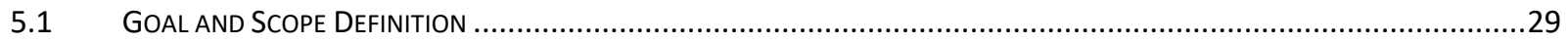

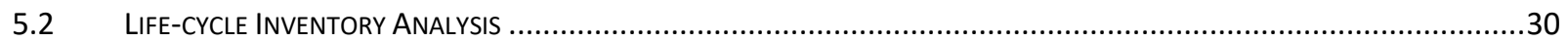

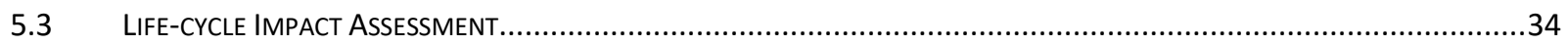

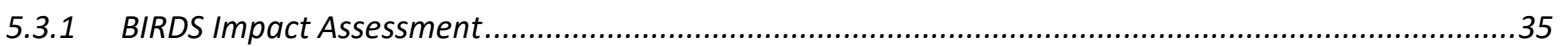

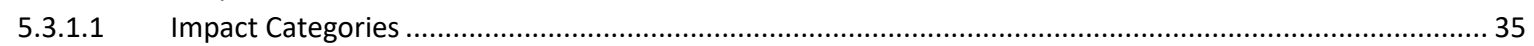

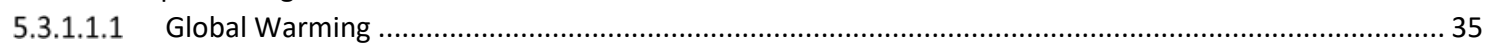

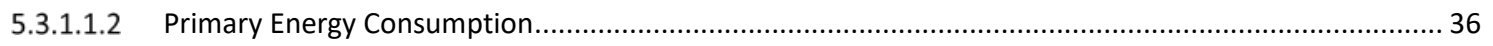

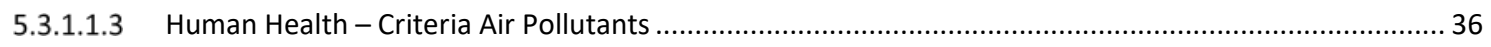

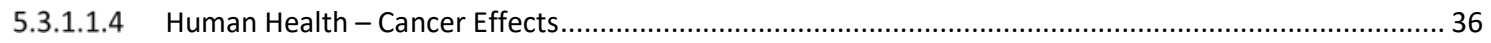

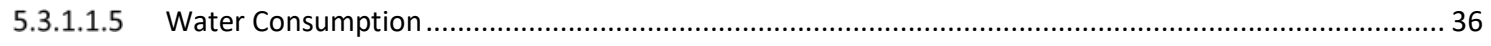

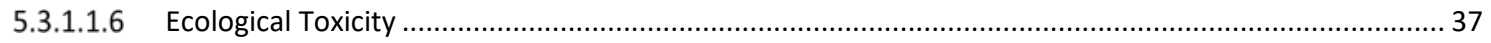

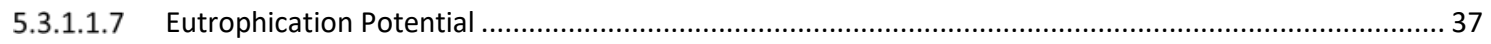

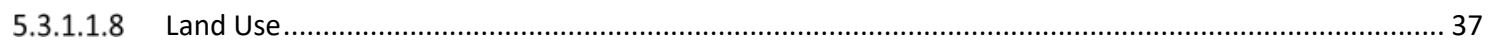

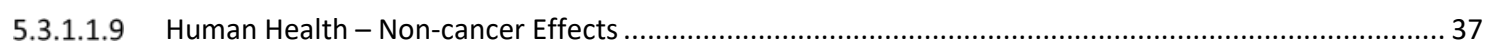

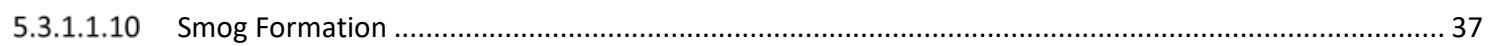

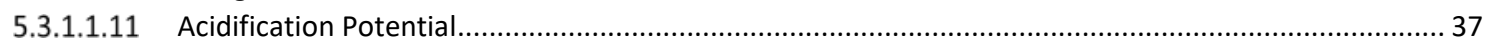

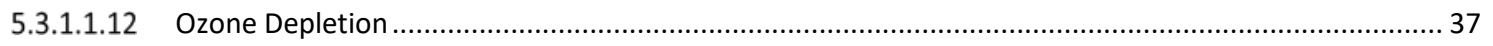




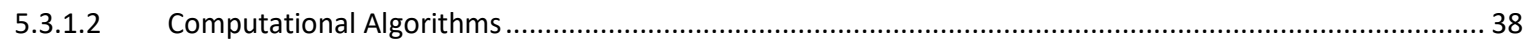

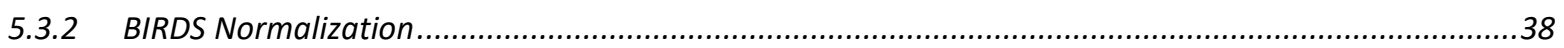

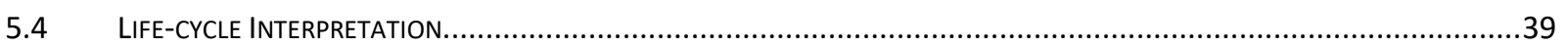

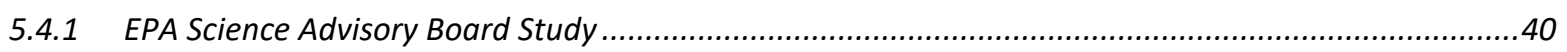

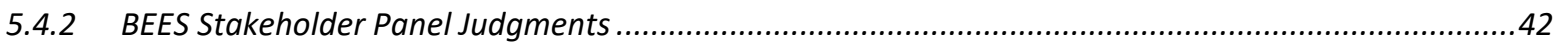

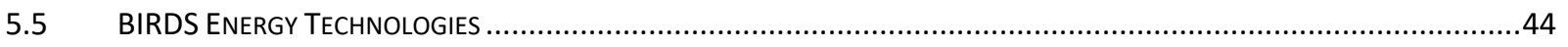

5.5.1 General Information Regarding the Energy Technology LCIs.................................................45

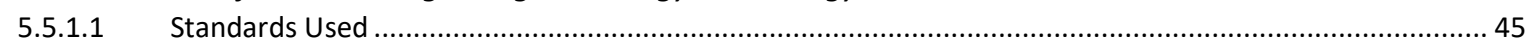

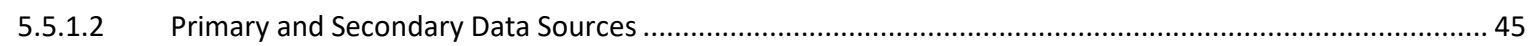

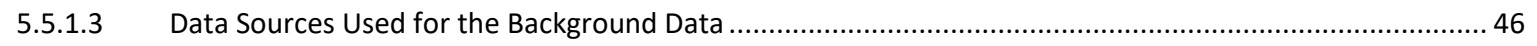

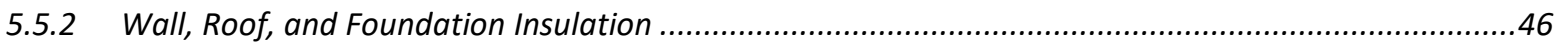

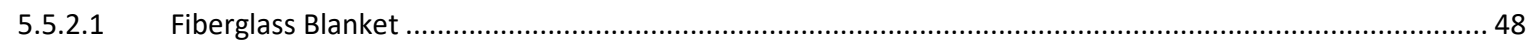

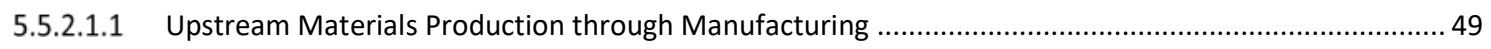

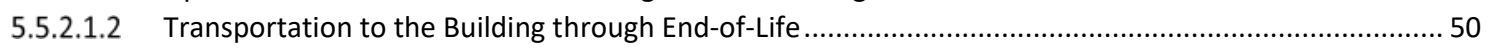

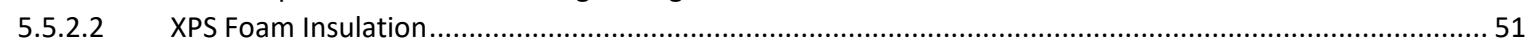

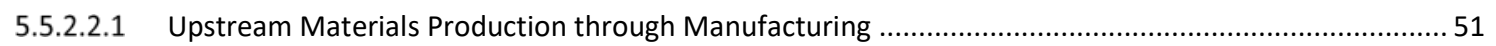

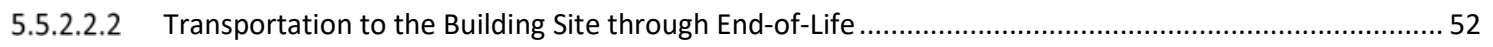

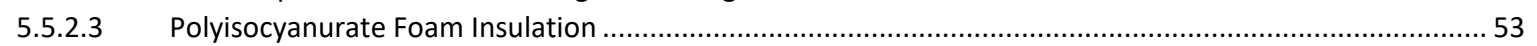

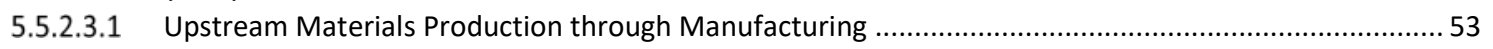

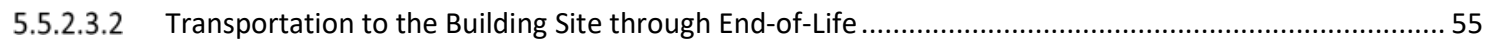

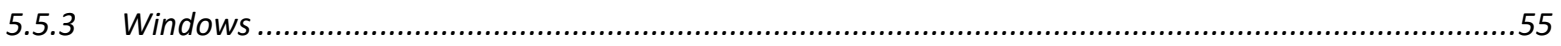

5.5.3.1 Window Upstream Materials Production through Manufacturing .........................................................5 58

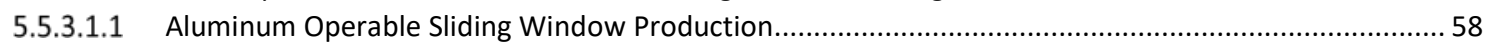

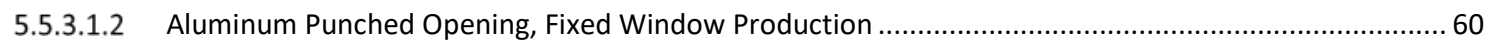

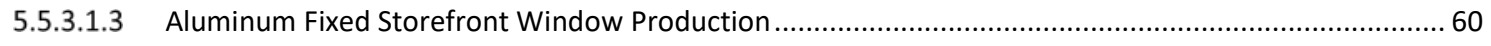

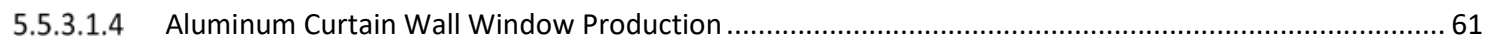

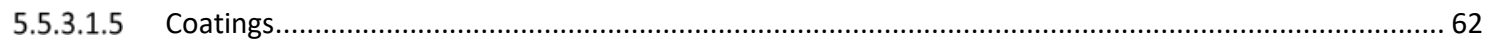

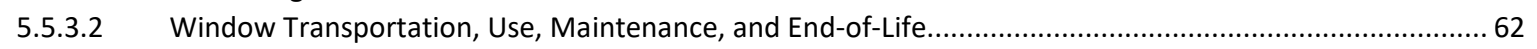

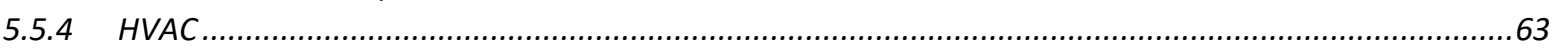

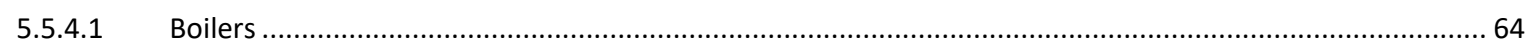

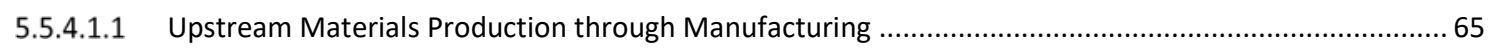

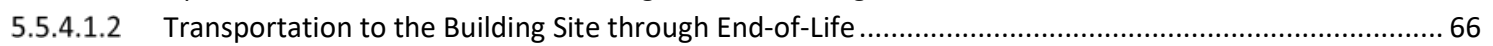

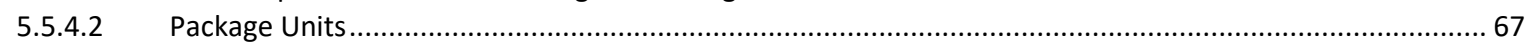

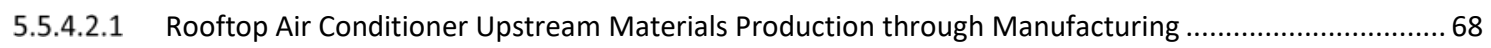

5.5.4.2.2 Other Package Units Upstream Materials Production through Manufacturing .....................................69

5.5.4.2.3 Air Cooled Condensing Unit - Split System Upstream Materials Production through Manufacturing ..... 70

5.5.4.2.4 Packaged Units Transportation to the Building Site through End-of-Life............................................ 71

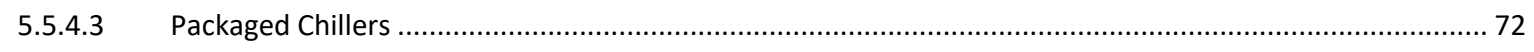

5.5.4.3.1 Air-Cooled Chillers - Upstream Materials Production .................................................................. 72

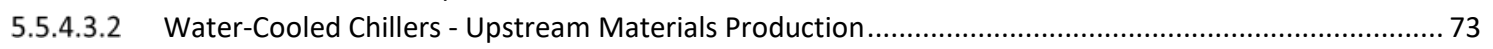

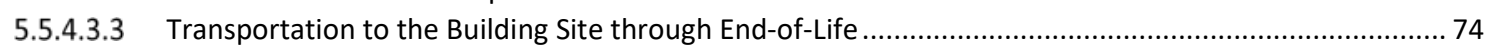

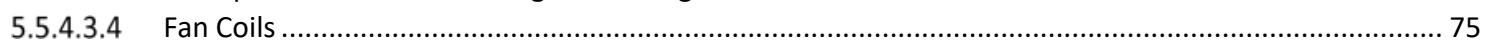

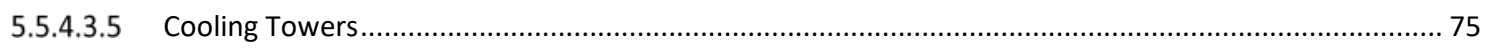

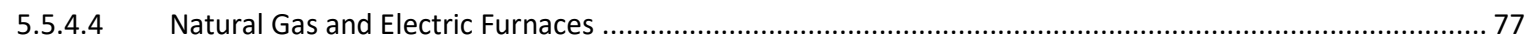

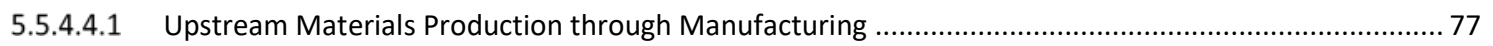

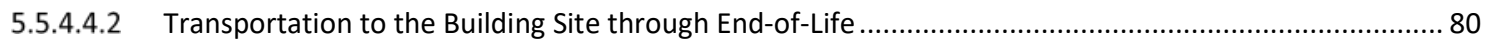

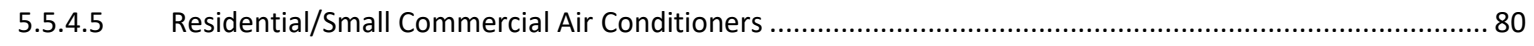

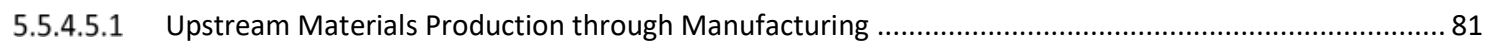

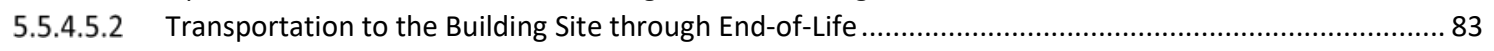

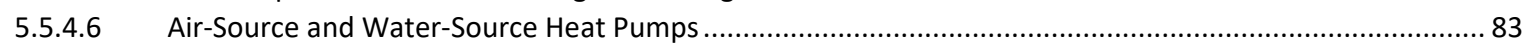

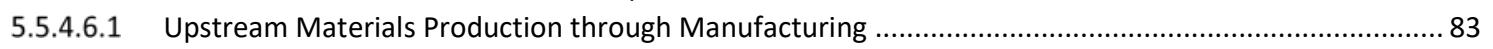

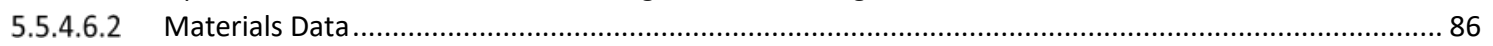

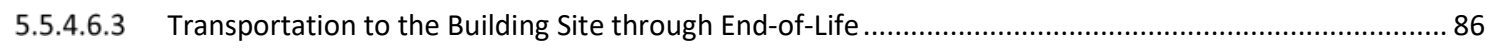




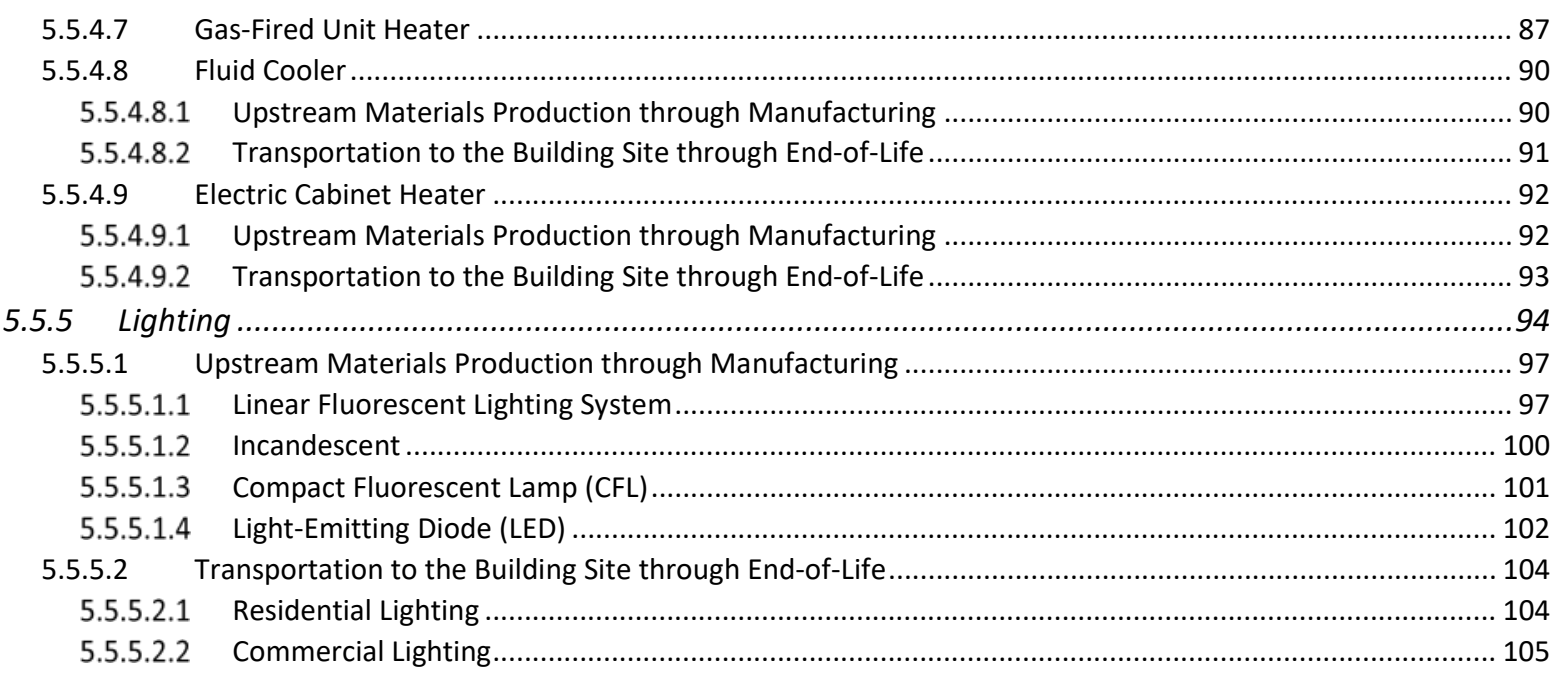

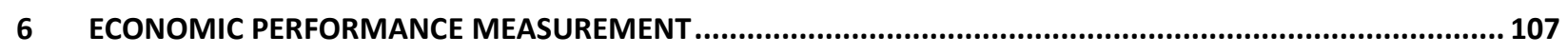

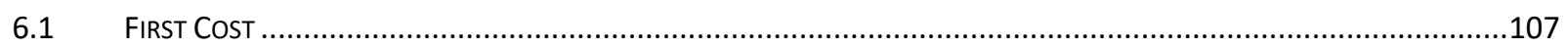

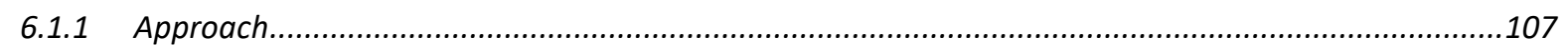

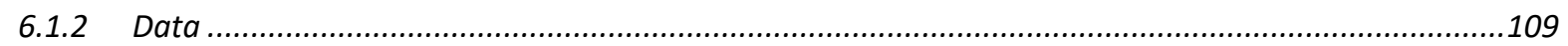

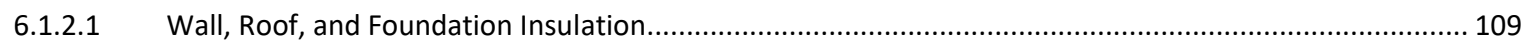

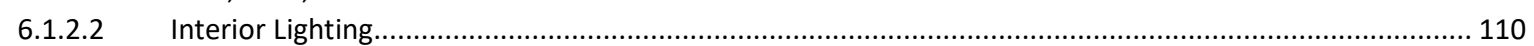

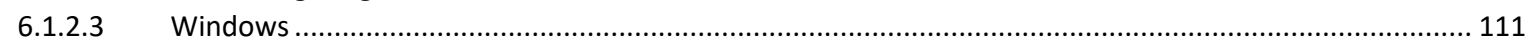

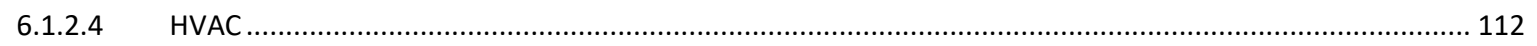

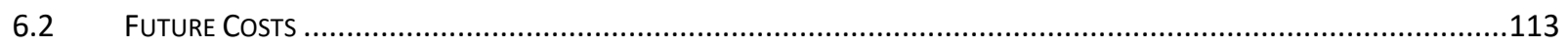

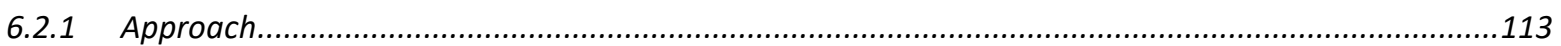

6.2.2 Data-Maintenance, Repair, and Replacement ................................................................114

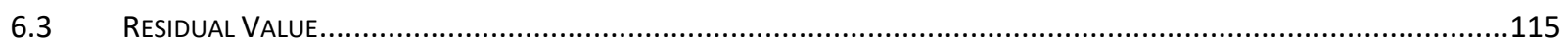

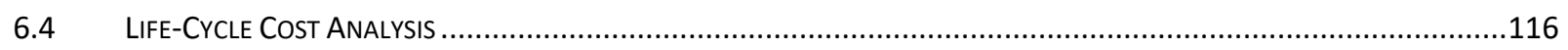

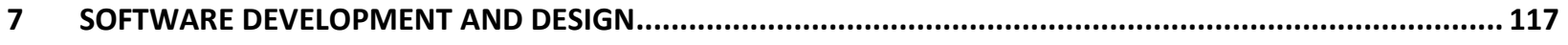

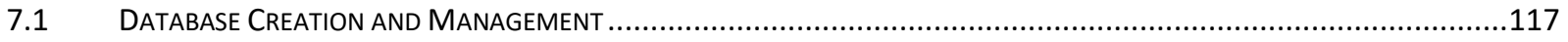

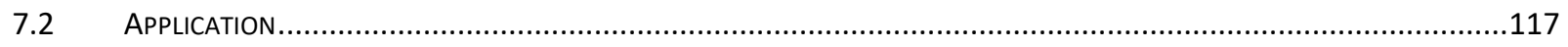

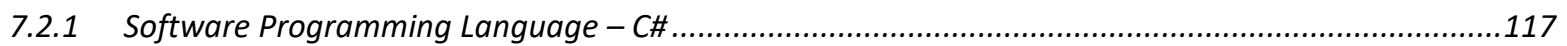

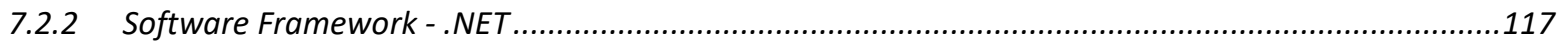

7.2.3 Web Development Technologies - HTML, CSS, JavaScript, JQuery, jqChart ..................................118

7.2.4 Application Design - Visual Studio....................................................................................118

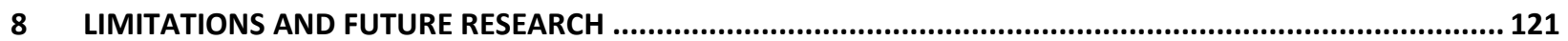

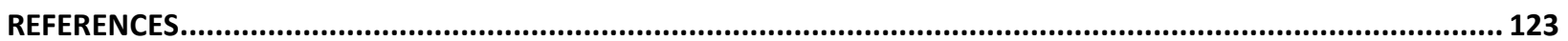




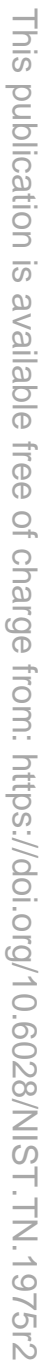




\section{List of Figures}

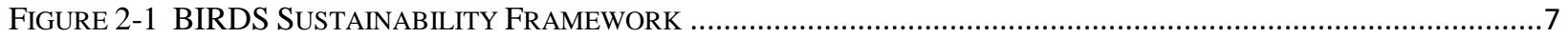

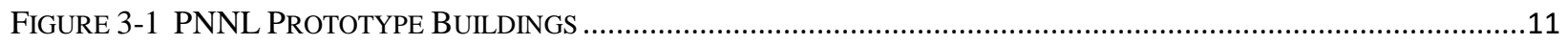

FIGURE 5-1 COMPILING LCA INVENTORIES OF ENVIRONMENTAL INPUTS AND OUTPUTS .......................................31

FIGURE 5-2 ILLUSTRATION OF SUPPLY CHAIN CONTRIBUTIONS TO U.S. CONSTRUCTION INDUSTRY .........................33

FIGURE 5-3 BEES STAKEHOLDER PANEL IMPORTANCE WEIGHTS SYNTHESIZED ACROSS VOTING INTEREST AND TIME

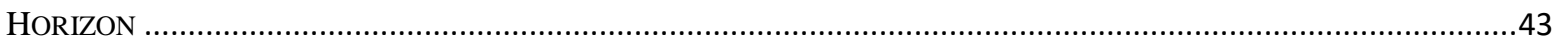

FIGURE 5-4 BEES STAKEHOLDER PANEL IMPORTANCE WEIGHTS BY STAKEHOLDER VOTING INTEREST ..................44

FIGURE 5-5 BEES STAKEHOLDER PANEL IMPORTANCE WEIGHTS BY TIME HORIZON ..........................................44

FIGURE 5-6 INSULATION SYSTEM BOUNDARIES - FIBERGLASS BLANKET EXAMPLE ............................................48

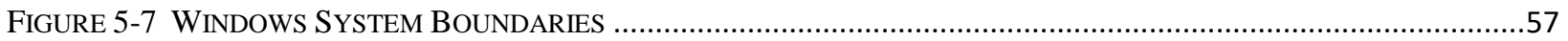

FIGURE 5-8 HVAC SYSTEM BOUNDARIES - BOILER EXAMPLE ...................................................................64

FIGURE 5-9 LIGHTING SYSTEM BOUNDARIES - CFL EXAMPLE ................................................................95

FIGURE 5-10 LINEAR FLUORESCENT LIGHTING SYSTEM BOUNDARIES.......................................................96

FIGURE 6-1 RSMEANS ONLINE SQUARE FOOT COST ESTIMATOR (SFCE) ...................................................108

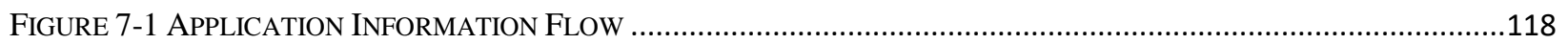




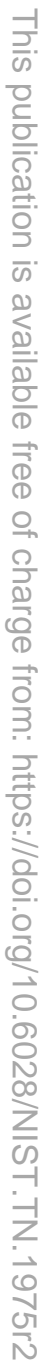




\section{List of Tables}

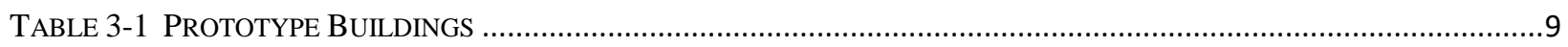

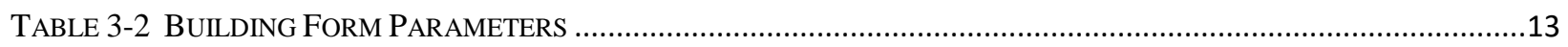

TABLE 3-3 BUILDING FABRIC SPECIFICATIONS: ROOF, EXTERIOR WALLS, AND FOUNDATION ................................14

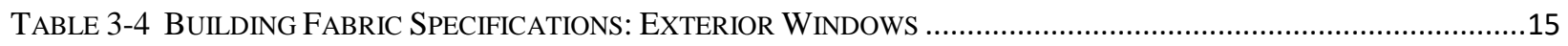

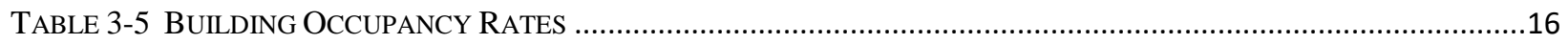

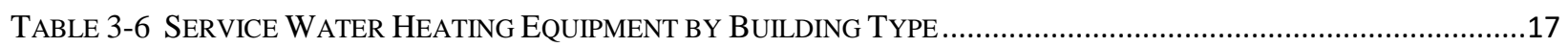

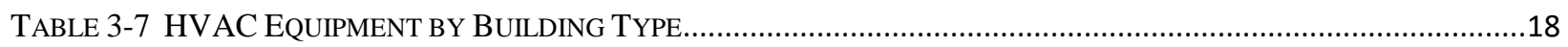

TABLE 3-8 HVAC EQUIPMENT MiNIMUM EFFICIENCIES - HEATING ........................................................................20

TABLE 3-9 HVAC EQUIPMENT MINIMUM EFFICIENCIES - COOLING.........................................................................2

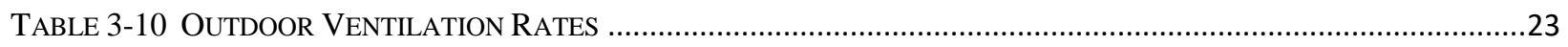

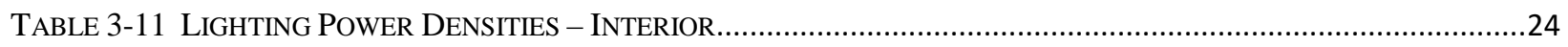

TABLE 4-1 ENERGY EFFICIENCY COMPONENT REQUIREMENTS FOR ALTERNATIVE BUILDING STANDARD EDITIONS

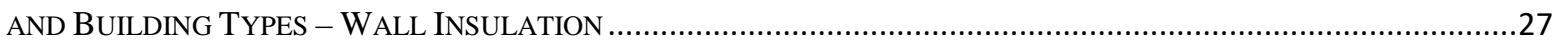

TABLE 4-2 ENERGY EFFICIENCY COMPONENT REQUIREMENTS FOR ALTERNATIVE BUILDING STANDARD EDITIONS

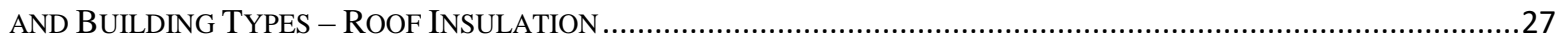

TABle 4-3 ENERgy EFFICIENCY COMPONENT REQUIREMENTS FOR ALTERNATIVE BUILDING STANDARD EDITIONS

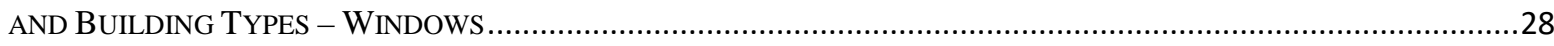

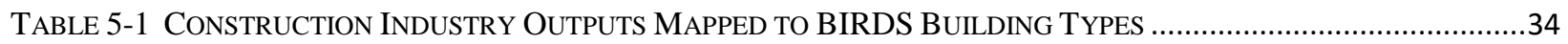

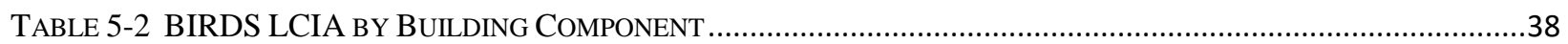

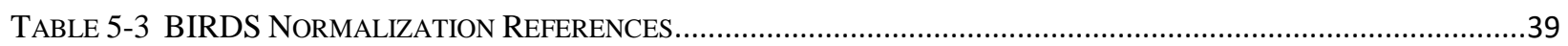

TABLE 5-4 PAIRWISE COMPARISON VALUES FOR DERIVING IMPACT CATEGORY IMPORTANCE WEIGHTS ...................41

TABLE 5-5 RELATIVE IMPORTANCE WEIGHTS BASED ON SCIENCE ADVISORY BOARD STUDY ..................................41

TABLE 5-6 RELATIVE IMPORTANCE WEIGHTS BASED ON BEES STAKEHOLDER PANEL JUDGMENTS ............................43

TABLE 5-7 SPECIFIED InSUlation TyPES AND R-VALUES - WALL APPLICATION …………....................................47

TABLE 5-8 SPECIFIED INSULATION TYPES AND R-VALUES - RoOF APPLICATION ….....................................................

TABLE 5-9 SPECIFIED INSUlation TYPES AND R-VALUES - FoundATION APPLICATION..........................................48

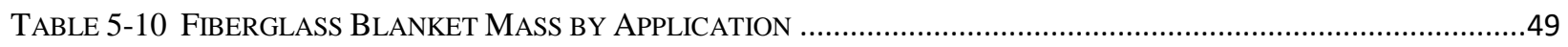

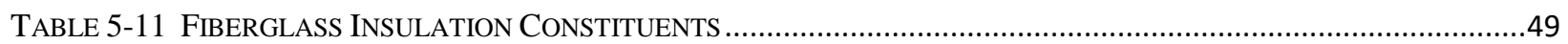

TABLE 5-12 ENERGY REQUIREMENTS FOR FIBERGLASS INSULATION MANUFACTURING ….....................................50

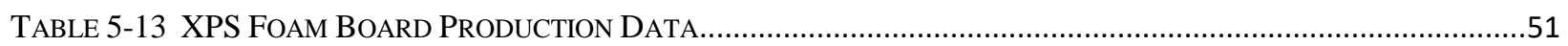

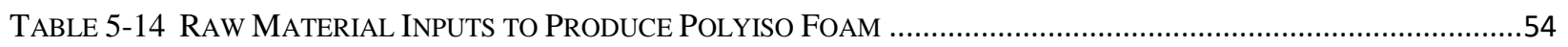

TABLE 5-15 ENERGY INPUTS AND PROCESS OUTPUTS FOR 1 BOARD-FOOT POLYISO FOAM .....................................55

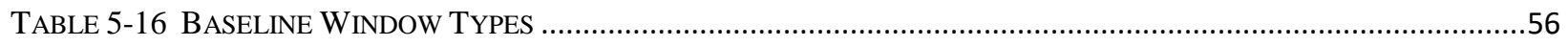

TABLE 5-17 CHARACTERISTICS AND COMPONENTS OF THE ALUMINUM OPERABLE WindOW .................................58

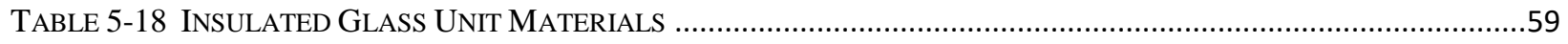

TABLE 5-19 CHARACTERISTICS AND COMPONENTS OF THE ALUMINUM FIXED WINDOW..........................................60

TABLE 5-20 CHARACTERISTICS AND COMPONENTS OF THE ALUMINUM STOREFRONT WINDOW ..................................61

TABLE 5-21 CHARACTERISTICS AND COMPONENTS OF THE ALUMINUM STOREFRONT WindOW ................................61

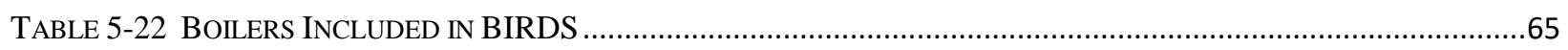

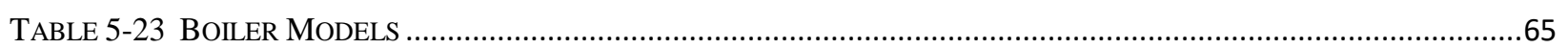

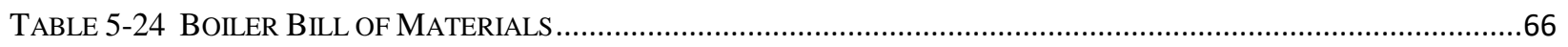

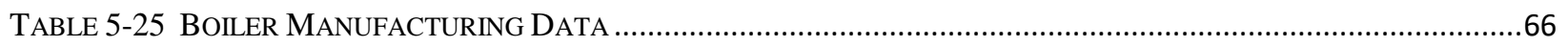

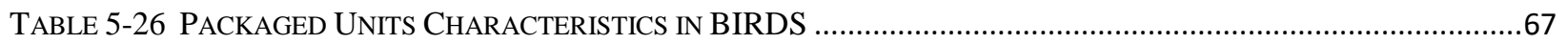

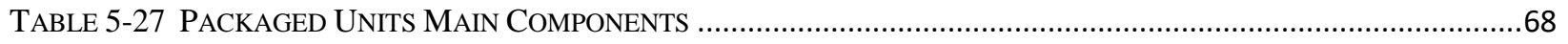

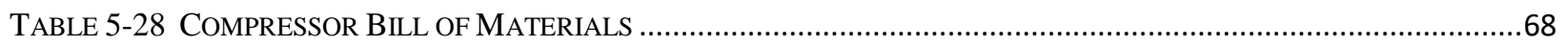

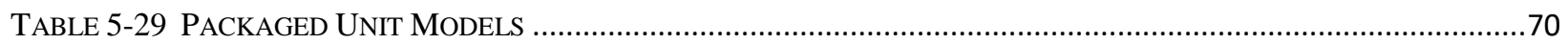

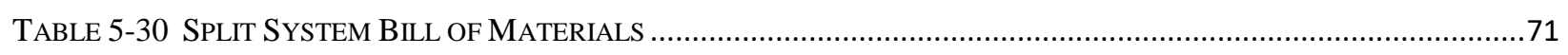




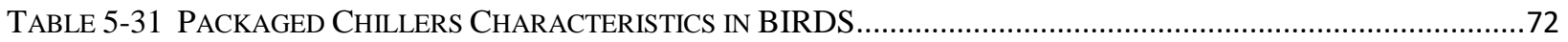

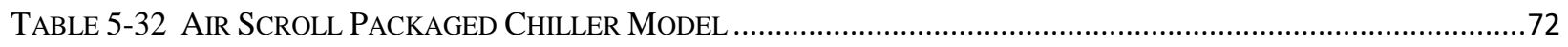

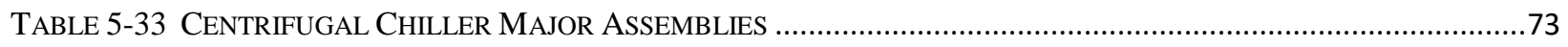

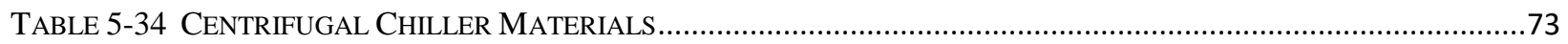



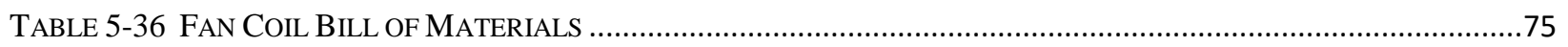

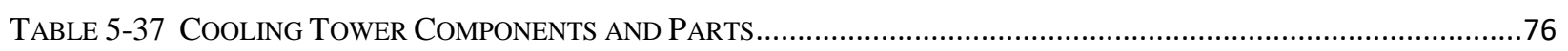

TABLE 5-38 COOLING TOWER BILL OF MATERIALS ……................................................................................

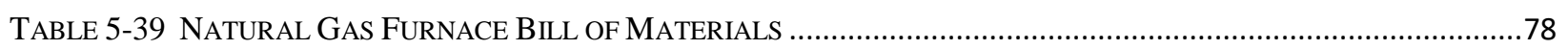

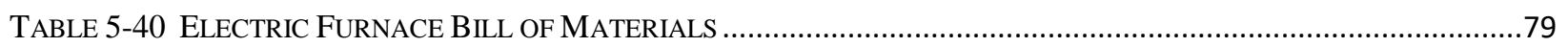

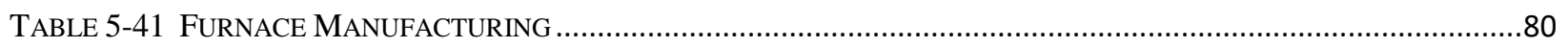

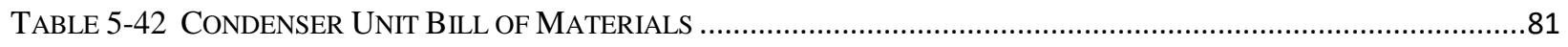

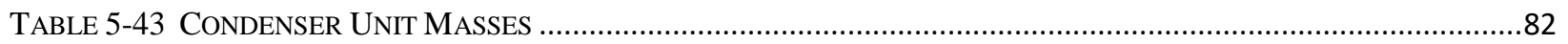

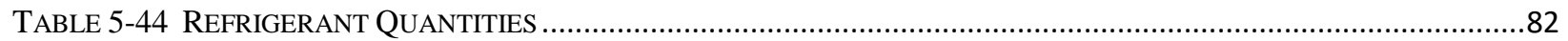

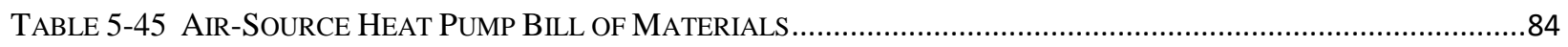

TABLE 5-46 WATER-SOURCE Heat PuMP Bill OF MaterialS...............................................................................8

TABLE 5-47 Water-Source Heat PumP PiPING Bill Of MAtERIALS ...............................................................8

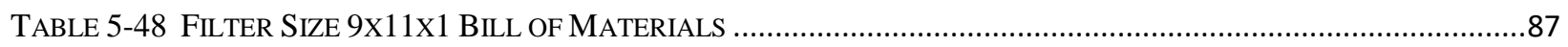

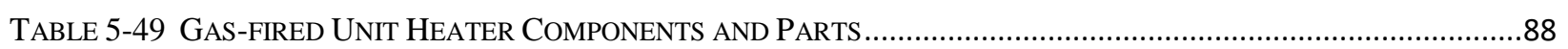



TABLE 5-51 AIR-COOLED FLUID COOLER COMPONENTS AND PARTS..................................................................90

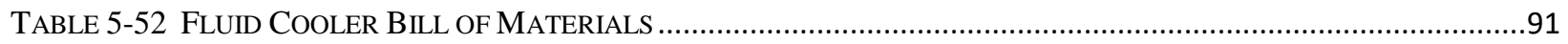

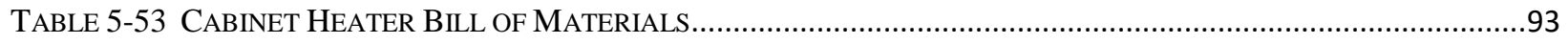

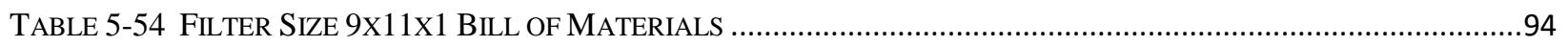

TABLE 5-55 PERFORMANCE OF LIGHTING TECHNOLOGIES IN BIRDS ................................................................95

TABLE 5-56 LINEAR FLUORESCENT LIGHT BULB CHARACTERISTICS ...................................................................96

TABLE 5-57 BuILDING Lighting USAGE AND REPLACEMENT RATES .....................................................................97

TABLE 5-58 FLUORESCENT BULB (TUBE) BILL OF MATERIALS ........................................................................98

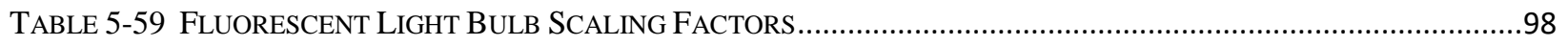

TABLE 5-60 ELECTRONIC BALLASt BILl OF MATERIALS AND PRODUCTION DATA .................................................99

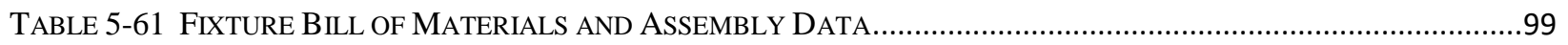

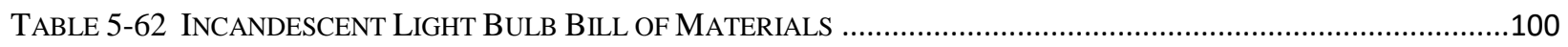

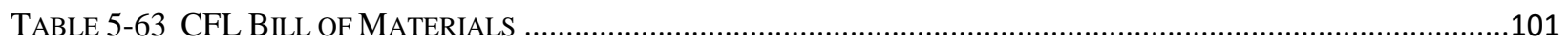

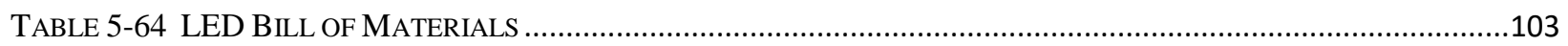

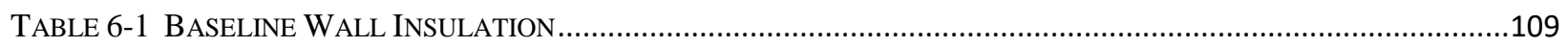

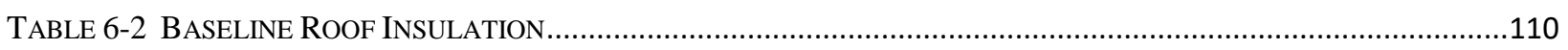

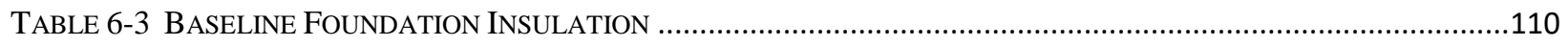

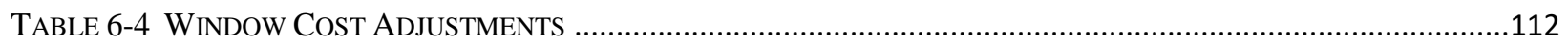

TABLE 6-5 2016 SPV DisCount FACTORS FOR Future NON-FuEL COSTS, 8 \% AND 3 \% REAL DisCount RATE...113 


\section{List of Acronyms}

\section{Acronym Definition}

ABS acrylontrile-butadiene-styrene

$\mathrm{ACH} \quad$ air changes per hour

AEO Applied Economics Office

AFUE Annual Fuel Utilization Efficiency

AHP Analytical Hierarchy Process

AHRI Air Conditioning, Heating, and Refrigeration Institute

AHS American Housing Survey

ASHRAE American Society of Heating, Refrigerating and Air-Conditioning Engineers

BARB Building America Research Benchmark

BEA Bureau of Economic Analysis

BEES Building for Environmental and Economic Sustainability

BIRDS Building Industry Reporting and Design for Sustainability

C\&D construction and demolition

CFA conditioned floor area

CFC-11 trichlorofluoromethane

CFL compact fluorescent lamp

CFM cubic feet per minute

$\mathrm{CO} 2$ carbon dioxide

CO2e carbon dioxide equivalent

COP coefficient of performance

E+ EnergyPlus

EER Energy Efficiency Ratio

EERE Office of Energy Efficiency \& Renewable Energy

eGDP environmental gross domestic product

EIA Energy Information Administration

EL Engineering Laboratory

ELA effective leakage area

EPA Environmental Protection Agency

EPD environmental product declaration

EPDM ethylene propylene diene monomer 


$\begin{array}{ll}\text { Acronym } & \text { Definition } \\ \text { GDP } & \text { gross domestic product } \\ \text { HBCD } & \text { hexabromocyclododecane } \\ \text { HCFC } & \text { hydrochlorofluorocarbon } \\ \text { HDPE } & \text { high density polyethylene } \\ \text { HFC } & \text { hydrochlorofluorocarbon } \\ \text { HSPF } & \text { heating seasonal performance factor } \\ \text { HVAC } & \text { heating, ventilation, and air-conditioning } \\ \text { IECC } & \text { International Energy Conservation Code } \\ \text { IGU } & \text { insulated glass unit } \\ \text { I-O } & \text { input-output } \\ \text { IPCC } & \text { Intergovernmental Panel on Climate Change } \\ \text { ISO } & \text { International Organization for Standardization } \\ \text { LBNL } & \text { Lawrence Berkeley National Laboratory } \\ \text { LCA } & \text { life-cycle assessment } \\ \text { LCC } & \text { life-cycle cost } \\ \text { LCI } & \text { life-cycle inventory } \\ \text { LCIA } & \text { life-cycle impact assessment } \\ \text { LED } & \text { light-emitting diode } \\ \text { Low-E } & \text { low-emissivity } \\ \text { M\&R } & \text { maintenance and repair } \\ \text { MBH } & \text { million Btu per hour } \\ \text { MDI } & \text { methylene diphenyl diisocyanate } \\ \text { MRR } & \text { maintenance, repair, and replacement } \\ \text { MSDS } & \text { material safety data sheet } \\ \text { NAHB } & \text { National Association of Home Builders } \\ \text { NIST } & \text { National Institute of Standards and Technology } \\ \text { NOx } & \text { Nirtous Oxide } \\ \text { NS } & \text { net savings } \\ \text { Net-Zero Energy Residential Test Facility } \\ \text { polybrominated diphenyl ethers } \\ \text { product category rules } \\ \text { PBE }\end{array}$




\section{Acronym Definition}

PIB polyisobutylene

PIMA Polyisocyanurate Insulation Manufacturers Association

PM10 particulate matter less than 10 micrometers in diameter

pMDI polymeric methylene diphenyl diisocyanate

PNS Net LCC savings as a percentage of base case LCC

PP propylene

PUR polyurethane

PV present value

PVC polyvinyl chloride

SAB Science Advisory Board

SEER seasonal energy efficiency ratio

SHGC solar heat gain coefficient

SPF spray polyurethane foam

SPFA Spray Poluurethane Foam Association

SPV single present value

SWH service water heating

TCPP tris(2-chloroisopropyl)phosphate

TRACI Tool for the Reduction and Assessment of Chemical and other environmental Impact

UPV* modified uniform present value discount factor

VOC volatile organic compound

VT visible transmittance

XPS extruded polystyrene

XPSA extruded polystyrene foam association 


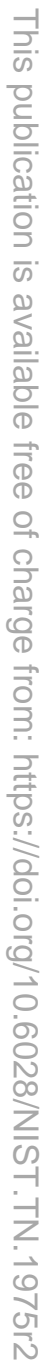




\section{Introduction}

\subsection{Purpose}

Building stakeholders need practical metrics, data, and tools to support decisions related to sustainable building designs, technologies, standards, and codes. The Engineering Laboratory (EL) of the National Institute of Standards and Technology (NIST) has addressed this high priority national need by extending its metrics and tools for sustainable building products, known as Building for Environmental and Economic Sustainability (BEES), to whole-buildings. Wholebuilding sustainability metrics have been developed based on innovative extensions to environmental life-cycle assessment (LCA) and life-cycle costing (LCC) approaches involving whole-building energy simulations. The measurement system evaluates the sustainability of both the materials and energy used by a building over time. It assesses the "carbon footprint" of buildings as well as 11 other environmental performance metrics and integrates economic performance metrics to yield science-based measures of the business case for investment choices in high-performance green buildings.

The approach previously developed for BEES has now been applied at the whole-building level to address building sustainability measurement in a holistic, integrated manner that considers complex interactions among building materials, energy technologies, and systems across dimensions of performance, scale, and time. Building Industry Reporting and Design for Sustainability (BIRDS) applies the sustainability measurement system to an extensive wholebuilding performance database NIST has compiled for this purpose. The energy, environment, and cost data in BIRDS provide measures of building operating energy use based on detailed energy simulations, building materials use through innovative life-cycle material inventories, and building costs over time. BIRDS v1.0 included energy, environmental, and cost measurements for 12540 commercial and non-low rise residential buildings, covering 11 building prototypes in 228 cities across all U.S. states for 9 study period lengths. See Lippiatt et al. (2013) for additional details. BIRDS v2.0 included both a commercial and residential database which incorporated the energy, environmental, and cost measurements for 9120 residential buildings, covering 10 single family dwellings ( 5 one-story and 5 two-story of various conditioned floor area) in 228 cities for study period lengths ranging from 1 year to 40 years.

BIRDS v3.0 incorporated the low-energy residential database with energy, environmental, and cost measurements. However, instead of considering locations across the country with minimal building design options, BIRDS v3.0 allowed for detailed incremental energy efficiency measure analysis for a single location, 240000 variations in residential building designs based on the NIST Net-Zero Energy Residential Test Facility (NZERTF) specifications and varying requirements across International Energy Conservation Code (IECC) editions. Again, study period lengths from 1 year to 40 years are included in the low-energy residential database. The sustainability performance of buildings designed to meet current energy codes can be compared to numerous alternative building designs to determine the impacts of improving building energy efficiency as well as varying the investor time horizon and other assumptions affecting overall sustainability performance. BIRDS v3.1 expanded the low-energy residential database including 
indoor environmental quality metrics based on occupant thermal comfort and indoor air quality (IAQ), as well as an alternative option for exterior wall finish that increased the number of residential building design variations by 480000 .

The latest version of BIRDS, v4.0, includes an update to all three databases (standards-based commercial database, code-based residential database, and incremental residential database). The updated database includes measurements for 17100 building designs, covering 15 building prototypes (13 commercial and 2 non-low rise residential) in 228 cities, for time horizons ranging from 1 year to 40 years. The commercial prototype buildings are based on models developed by the Pacific Northwest National Laboratory (PNNL), referred to as Commercial Prototype Building Models, and are representative of roughly $80 \%$ of newly constructed floor area in the United States. The previous residential database included costs for building construction, operation, maintenance, repair, and replacement in year 2014 dollars (\$2014). The updated database includes costs for similar buildings-related cost components, but in year 2017 dollars (\$2017). The updated low-energy residential database, now renamed the BIRDS LowEnergy Residential Incremental Energy Efficiency Improvements Database, has expanded the building component combinations to 960000 by adding options for natural gas-fired space and water heating equipment.

\subsection{Background}

A wave of interest in sustainability gathered momentum in 1992 with the Rio Earth Summit, during which the international community agreed upon a definition of sustainability in the Bruntland report: "meeting the needs of the present generation without compromising the ability of future generations to meet their own needs" (Brundtland Commission 1987). In the context of sustainable development, needs can be thought to include the often-conflicting goals of environmental quality, economic well-being, and social justice. While the intent of the 1992 summit was to initiate environmental and social progress, it seemed to have instead brought about greater debate over the inherent conflict between sustainability and economic development (Meakin 1992) that remains a topic of discussion to this day.

This conflict is particularly apparent within the construction industry. Demand for "green" and "sustainable" products and services have grown exponentially over the last decade, leading to 2.5 million "Green Goods and Services" private sector jobs and 886000 public sector jobs for a total of 3.4 million across the United States. Of these, a million are in the manufacturing and construction industries (Bureau of Labor Statistics 2013). There are nearly 600 green building product certifications, including nearly 100 used in the U.S. (National Institute of Building Sciences 2017), all of which use their own set of criteria for evaluating "green/sustainable" products. Also, the green building segment of the US market has grown $1700 \%$ from market share of $2 \%$ in 2005 to $38 \%$ in 2011 (Green America 2013), and was $67 \%$ of all projects in 2015 (McGraw-Hill Construction 2017). Projections of green construction spending growth show a rise from $\$ 151$ billion in 2015 to $\$ 224$ billion by 2018, leading to total impacts on US GDP of \$284 billion (U.S. Green Building Council 2015). Similar trends are occurring internationally as well, with over 100000 USGBC LEED projects (completed or in progress) and 200000 LEED professionals in 162 countries (U.S. Green Building Council 2017). 
Well-intentioned green product purchasing, green building design selection, and green development plans may not be economically competitive, and economic development plans may fail to materialize over concerns for the environment and public health. Thus, an integrated approach to sustainable construction - one that simultaneously considers both environmental and economic performance - lies at the heart of reconciling this conflict.

Interest in increasing energy efficiency across the U.S. building stock has been revived in the past decade as fluctuations in fossil fuel prices have increased and an increasing awareness and concern over potential climate change impacts has driven the public away from traditional energy sources. Buildings account for $40 \%$ of all energy consumed in the U.S. Given the costeffectiveness of energy efficiency improvements, buildings have become the target of numerous efforts to reduce domestic energy use. For this reason, the BIRDS approach considers both the environmental and economic dimensions of sustainability with respect to building energy efficiency. BIRDS, however, does not consider the social dimension of sustainability (e.g. livability, resilience) now due to the current lack of applicable rigorous measurement methods. As methods develop and continue to improve over time, elements of social sustainability will be incorporated into BIRDS. 


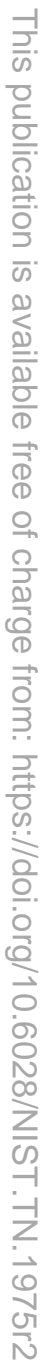




\section{BIRDS Approach}

\subsection{BIRDS Sustainability Measurement}

One standardized and preferred approach for scientifically measuring the environmental performance of industrial products and systems is life-cycle assessment (LCA). LCA is a "cradle-to-grave" systems approach for measuring environmental performance. The approach is based on two principles. First, the belief that all stages in the life of a product generate environmental impacts and must be analyzed, including raw materials acquisition, product manufacture, transportation, installation, operation and maintenance, and ultimately recycling and waste management. An analysis that excludes any of these stages is limited because it ignores the full range of upstream and downstream impacts of stage-specific processes. LCA broadens the environmental discussion by accounting for shifts of environmental problems from one life-cycle stage to another. The second principle is that multiple environmental impacts must be considered over these life-cycle stages to implement a trade-off analysis that achieves a genuine reduction in overall environmental impact, rather than a simple shift of impact. By considering a range of environmental impacts, LCA accounts for impact-shifting from one environmental medium (land, air, water) to another.

The LCA method is typically applied to products, or simple product assemblies, in a "bottom up" manner. The environmental inputs and outputs to all the production processes throughout a product's life-cycle are compiled. These product life-cycle "inventories" quantify hundreds, even thousands, of environmental inputs and outputs. This is a data-intensive, time-consuming, and expensive process that must be repeated for every product.

The bottom-up approach becomes unwieldy and cost prohibitive for complex systems, such as buildings, that involve potentially hundreds of products. Furthermore, a building's sustainability is not limited to the collective sustainability of its products. The way designers integrate these products and systems at the whole-building level has a large influence on another major dimension of its sustainability performance, operating energy use.

The BIRDS model applies a unifying LCA framework developed for the U.S. economy to the U.S. construction sector and its constituent building types. Through this “top-down” LCA approach, a series of baseline sustainability measurements are made for prototypical buildings, yielding a common yardstick for measuring sustainability with roots in well-established national environmental and economic statistics. Using detailed "bottom-up” data compiled through traditional LCA approaches, the baseline measurements for prototypical buildings are then "hybridized" to reflect a range of improvements in building energy efficiency, enabling assessment of their energy, environmental, and economic benefits and costs. The idea is to provide a cohesive database and measurement system based on sound science that can be used to prioritize green building issues and to track progress over time as design and policy solutions are implemented. "Bottom-up” and "top-down" data sources and approaches will be discussed further in Chapter 4. 
The BIRDS hybrid LCA approach combines the advantages of both the bottom-up and top-down approaches-namely the use of higher-resolution, bottom-up data and the use of regularly-updated, top-down statistical data without truncation (Suh, Lenzen et al. 2004, Suh and Huppes 2005). The hybrid approach generally reduces the uncertainty of existing pure bottom-up or pure top-down systems by reducing truncation error in the former and increasing the resolution of the latter (Suh, Lenzen et al. 2004). The hybrid approach will be discussed in further detail in Chapter 4.

Operating energy use - a key input to whole-building LCAs-is assessed in BIRDS using the bottom-up approach. Energy use is highly dependent upon a building's function, size, location, and the efficiency of its energy technologies. Energy efficiency requirements in current energy codes for commercial buildings vary across states, and many states have not yet adopted the newest energy standard editions. As of January 1, 2017, state energy code adoptions range across all editions of the American Society of Heating, Refrigerating, and Air-Conditioning Engineers Energy (ASHRAE) Energy Standard for Buildings except Low-Rise Residential Buildings (ASHRAE 90.1-2004, -2007, -2010, and -2013) and the International Energy Conservation Code (IECC) for Commercial Buildings (2006, 2009, 2012, and 2015). Some states do not have a code requirement for energy efficiency, leaving it up to the locality or jurisdiction to set its own requirement. To address these issues, operating energy use in BIRDS is tailored to commercial and non-low rise residential building types, locations, and energy codes. The BIRDS database includes operating energy use predicted through energy simulation of 4 alternative building designs for 15 building types in 228 U.S. locations, with each design complying with some version of ASHRAE 90.1.

Often, the many dimensions of a building's environmental performance are ultimately balanced against its economic performance. However, studies have shown that energy efficient commercial buildings or those with green attributes realize increased resale value (2 to17 \%), rental rates (5.8 \% to $35 \%$ ), occupancy rates ( $0.9 \%$ to $18 \%$ ), net operating income $(5.9 \%)$, and productivity (4.8\%) while lowering operating expenses (30\%) and capitalization rates (50 to 55 basis points) (Institute for Building Efficiency 2012). ${ }^{1}$ Similarly, residential building owners have some willingness to pay for energy efficiency and green certified homes. A 2006 poll by the American Institute of Architects showed that $90 \%$ of U.S. consumers would be willing to pay more to reduce their home's environmental impact, but only an additional $\$ 4000$ to $\$ 5000$, or about $2 \%$, more. ${ }^{2}$ More recent studies have shown that U.S. home buyers are willing to pay more for sustainable building designs or homes with green attributes like solar photovoltaic (PV) (Griffin, Kaufman et al. 2009, Hoen 2011, Pfleger, Perry et al. 2011, Aroul and Hansz 2012, Dastrup, Zivin et al. 2012, Kok and Kahn 2012, Kahn and Kok 2014, Adomatis 2015). There are significant variations across locations in the value placed on green-rated homes, which may be driven by consumer preferences or knowledge. To satisfy stakeholders, the green building

\footnotetext{
${ }^{1}$ Meta-analysis of Eicholtz et al. (2009), Eicholtz et al. (2010), Fuerst and McAllister (2009), Fuerst and McAllister (2009), Kats et al. (2003), Kok and Jennen (2011), Loftness et al. (2003), Miller et al. (2008), Miller et al. (2009), Pivo and Fisher (2009), and Wiley et al. (2010).

${ }^{2}$ January 2006 survey cited in Green Buildings in the Washington Post (Cohen 2006).
} 
community needs to promote and design buildings with an attractive balance of environmental and economic performance. These considerations require innovative means to address sustainability performance for buildings (Eichholtz, Kok et al. 2010).

Like operating energy use, a building's economic performance is dependent upon a building's function, location, and the efficiency of its energy technologies. Construction material and labor costs vary by building type and location, as do maintenance, repair, and replacement costs over time. Energy technologies for compliance with a given ASHRAE energy standard edition vary across U.S. climate zones, as do their costs. Finally, a building's operating energy costs vary per the quantity and price of energy use, which depend upon the building's location and fluctuate over time. All these variables are accounted for in the BIRDS database, as shown in Figure 2-1.

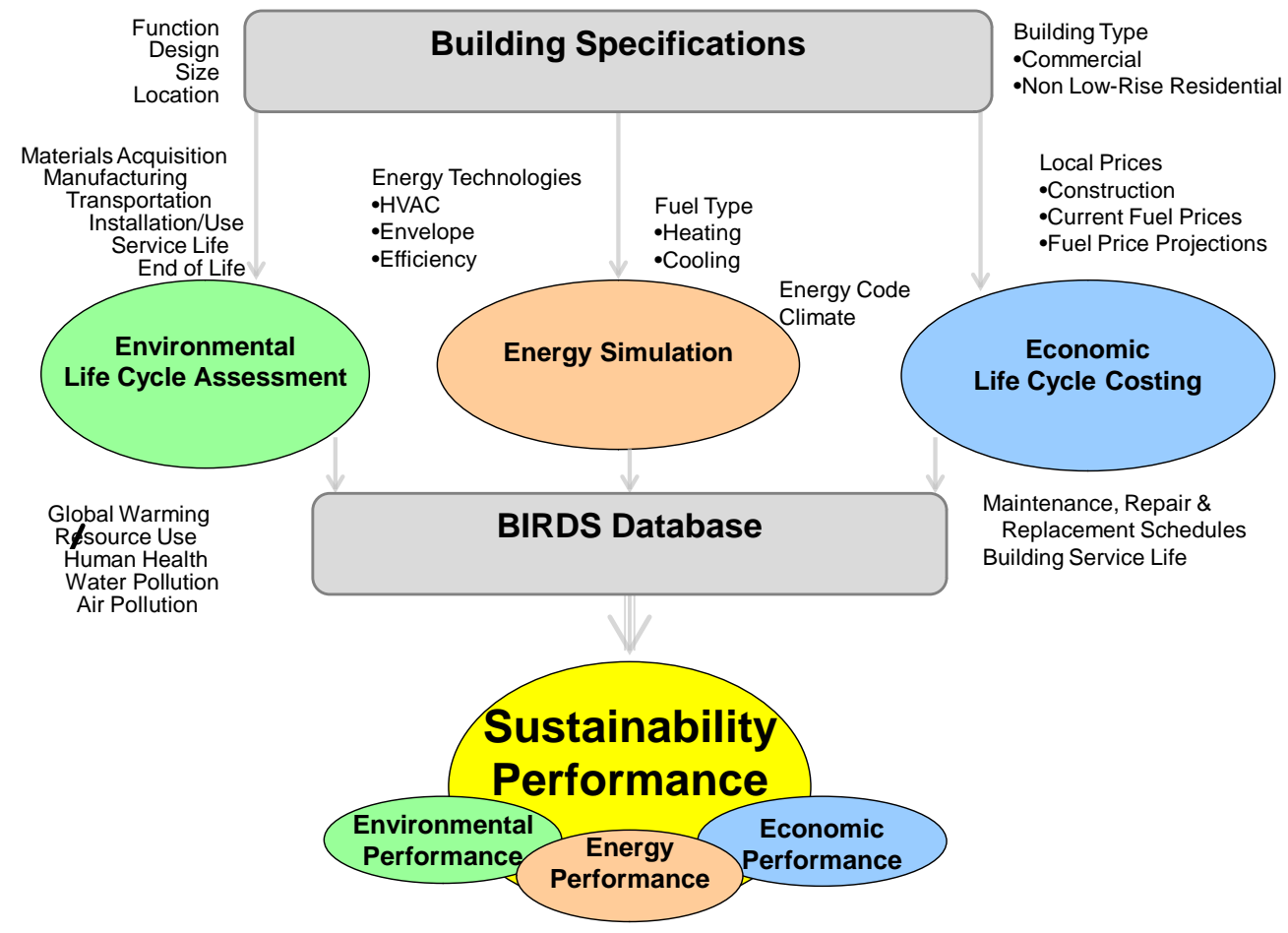

Figure 2-1 BIRDS Sustainability Framework

\subsection{Establish Consistency}

Measuring building sustainability performance in BIRDS requires that special attention be paid to establishing consistency among its many dimensions. While BIRDS develops separate performance metrics for building energy, environmental, and economic performance, they are all developed using the same parameters and assumptions. For each of the 13680 building designs included in the updated BIRDS commercial database, consistent design specifications are used to estimate its operating energy use, environmental life-cycle impacts, and life-cycle costs. The building energy simulation, for example, specifies the same building envelope and heating, 
ventilation, and air-conditioning (HVAC) technologies as do the bottom-up energy technology LCAs and cost estimates.

One of the most important dimensions requiring BIRDS modeling consistency is the study period. The study period is the number of years of building operation over which energy, environmental, and economic performance are assessed. In economic terms, the study period represents the investor's time horizon. Over what timeframe are investors or policymakers interested in the environmental and economic costs and benefits related to the capital investment decision? Since different stakeholders have different time perspectives, there is no one correct study period for developing a business case for sustainability. For this reason, 40 different study period lengths are offered in BIRDS, ranging from 1 year to 40 years.

Forty study period lengths are chosen to represent the wide cross section of potential investment time horizons. A 1-year study period is representative of a developer that intends to sell a property soon after it is constructed. A 5-year to 15-year study period best represents a building owner's time horizon because few owners are concerned about costs realized beyond a decade into the future. The 20-year to 40-year study periods better represents institutions, such as colleges or government agencies, because these entities will own or lease buildings for 20 or more years. BIRDS sets the maximum study period at 40 years for consistency with requirements for federal building life-cycle cost analysis (U.S. Congress 2007). Beyond 40 years, technological obsolescence becomes an issue, data become too uncertain, and the present value cost implications become less important.

Once the BIRDS user sets the length of the study period, the energy, environmental, and economic data are all normalized to that study period. This involves adjustments to a building's operating, maintenance, repair, and replacement data as well as to its residual value at the end of the study period. This assures consistency and comparability among the three metrics and is one of the strengths of the BIRDS approach.

The next four chapters present more detail regarding the modeling of the prototype buildings, energy, environmental, and economic performance measures within the updated BIRDS commercial building database. 


\section{Commercial Prototype Buildings}

Sixteen prototype commercial buildings were developed by the Pacific Northwest National Laboratory (PNNL) that are largely based on the commercial reference building models developed by the U.S. Department of Energy (DOE) Building Technologies program. These models served as starting points for research in building energy efficiency as they reflected the most common, newly constructed commercial buildings in the United States. The prototype models were originally developed in EnergyPlus (E+), and more recently OpenStudio (OS) includes measures to generate these building models. Unlike the original DOE Commercial Reference Buildings, which represented $70 \%$ of new commercial construction, the 16 PNNL building models collectively represent $80 \%$ of new commercial building stock. The PNNL collection of models also switch out the supermarket model for a new building prototype depicting a typical high-rise apartment building. Table 3-1 lists the 15 PNNL prototype buildings included in BIRDS. ${ }^{3}$ For additional information on the PNNL prototype buildings, please refer to (Thornton, Rosenberg et al. 2011). Building service lifetimes are assumed to be 41 years for all commercial prototype buildings and 65 years for all residential prototype buildings (Lufkin, Abate et al. 2010), which ensures that each building prototype lasts through the longest potential study period (40 years).

\section{Table 3-1 Prototype Buildings}

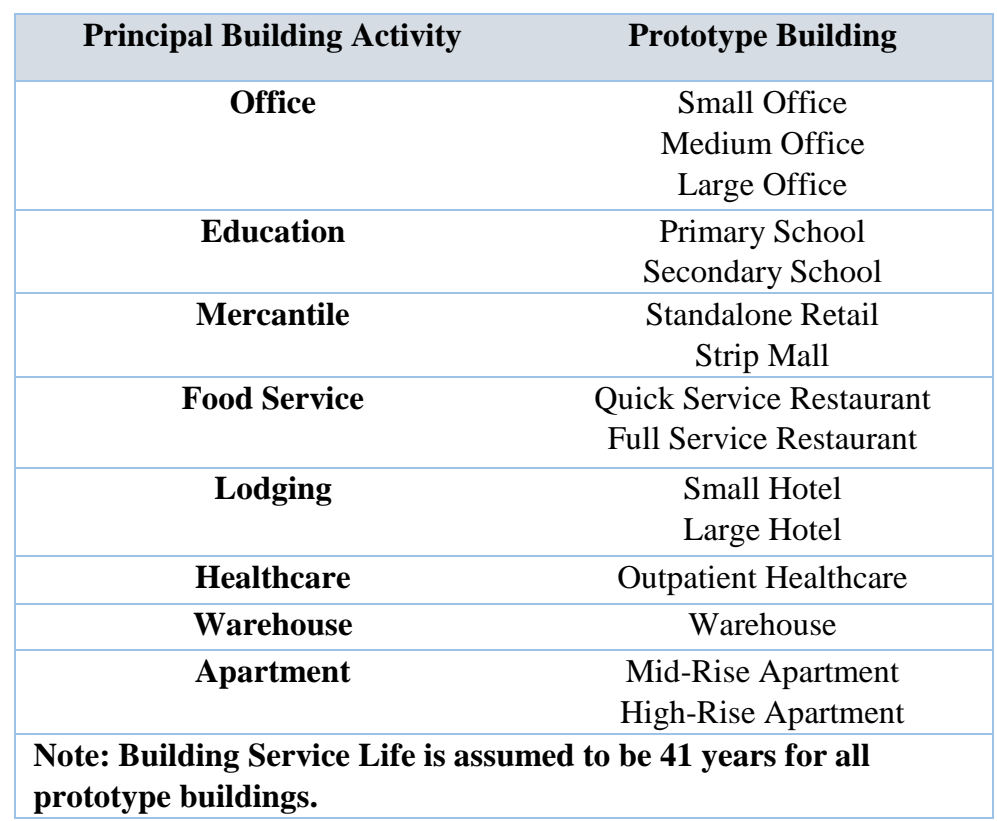

Given the complexity of its building systems, the new commercial database excludes the hospital prototype building and focuses only on the remaining 15 (Figure 3-1). The sections that follow

\footnotetext{
${ }^{3}$ The Hospital is not included in BIRDS given the complexities of its systems and lack of an OpenStudio model for the prototype building.
} 
provide background on the form, fabric, occupancy specifications, service water heating, HVAC systems, and lighting for each of the 15 prototype buildings included in BIRDS v4.0. ${ }^{4}$

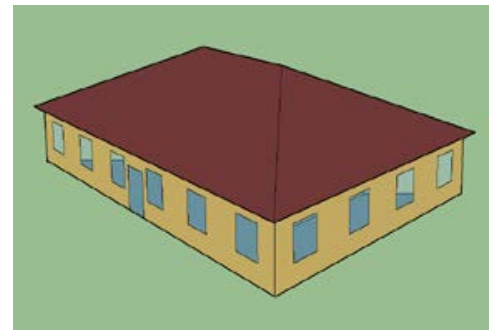

Small Office

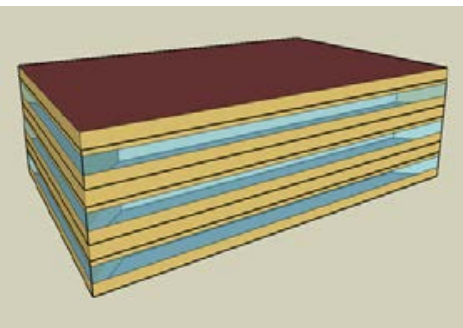

Medium Office

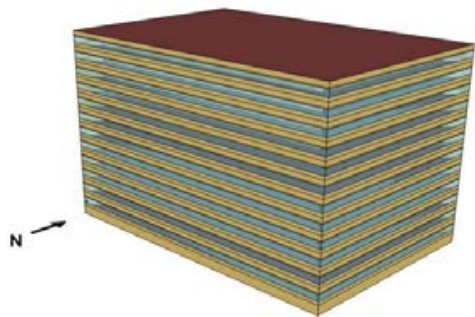

Large Office

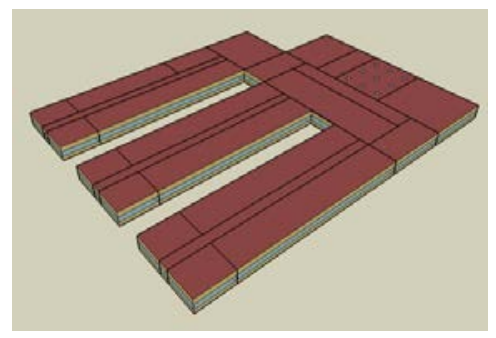

Primary School

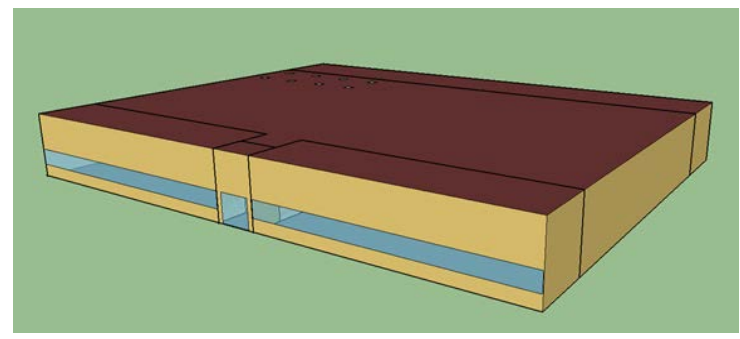

Standalone Retail

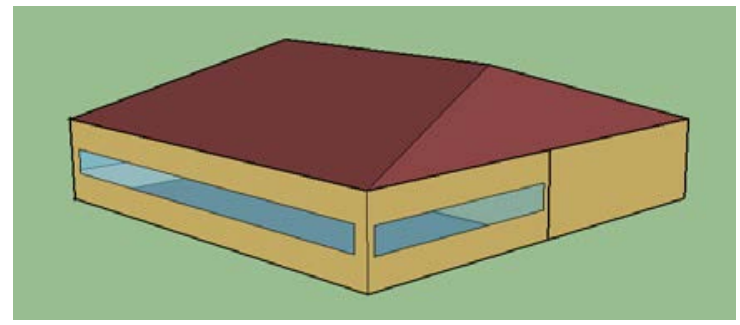

Fast Food Restaurant

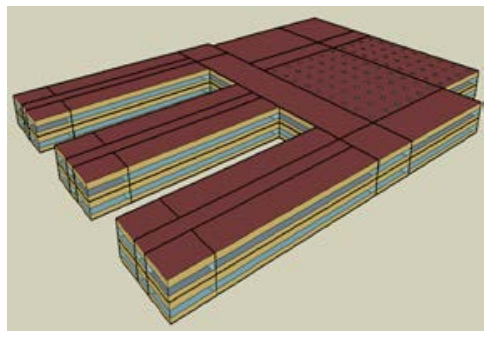

Secondary School

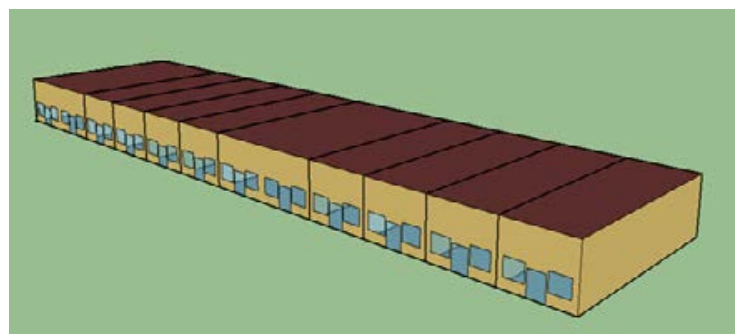

Strip Mall

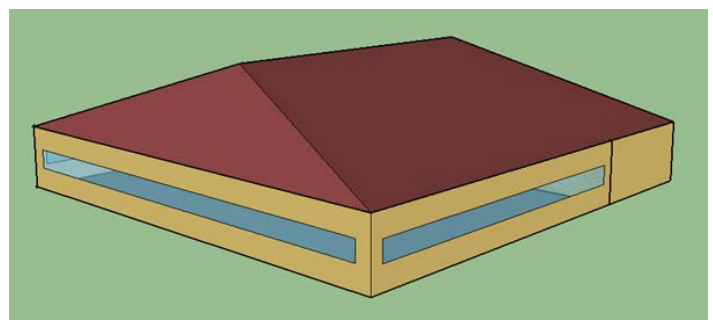

Sit Down Restaurant

${ }^{4}$ Roofing surfaces are shaded in brown. Exterior wall surfaces are shaded in yellow, while all fenestration surfaces are shaded in light blue. 


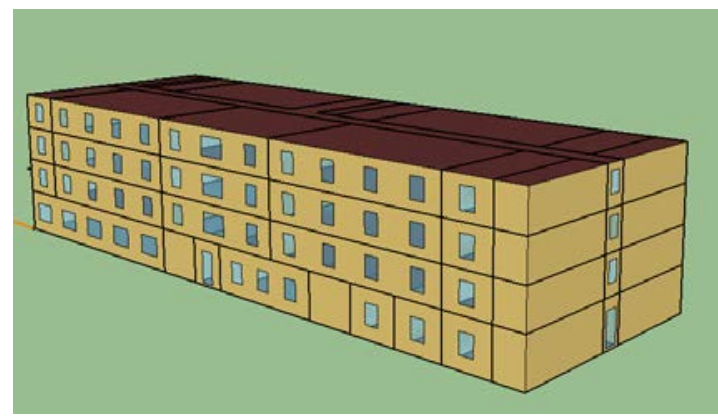

Small Hotel

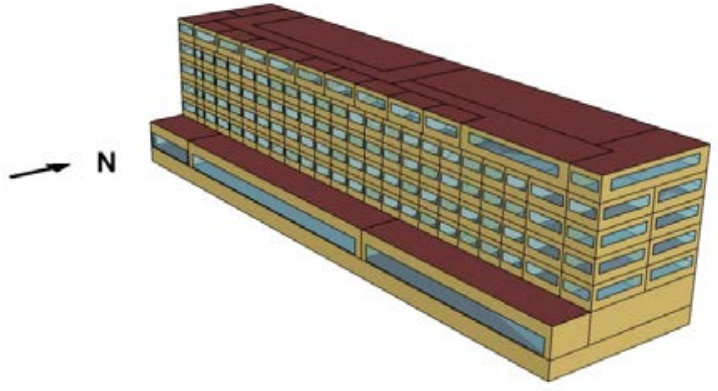

Large Hotel

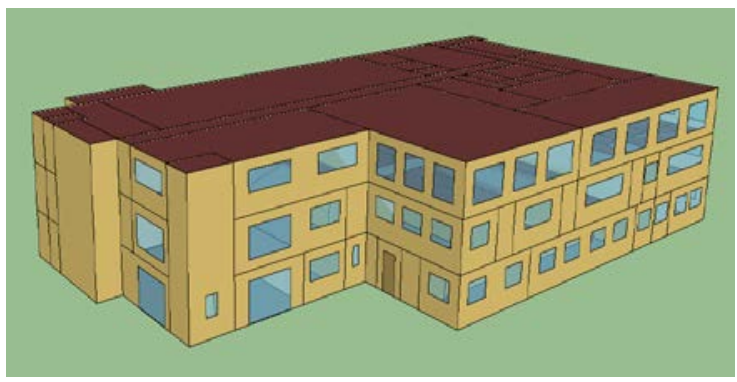

Outpatient Healthcare

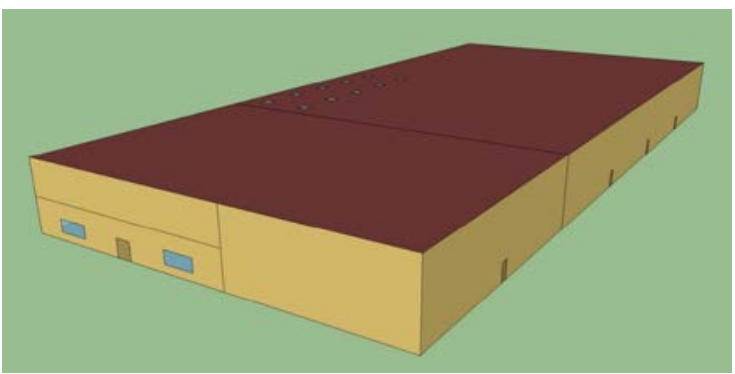

Warehouse

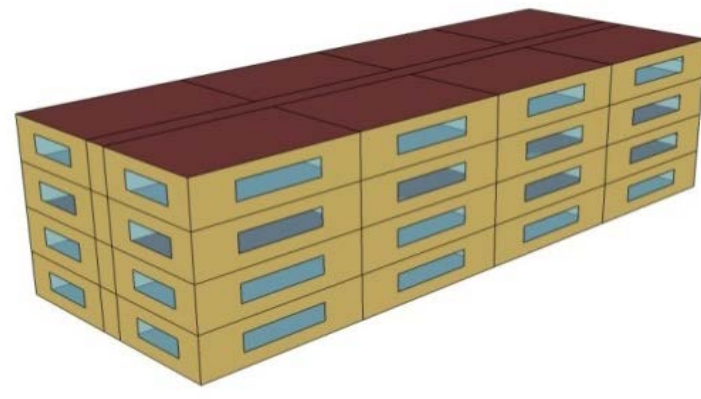

Mid-rise Apartment

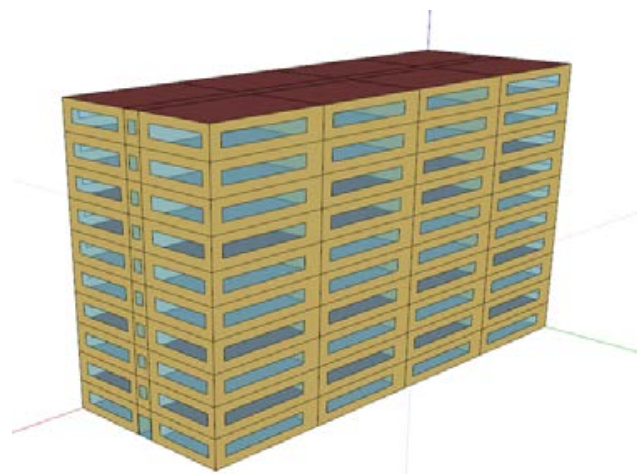

High-rise Apartment

Figure 3-1 PNNL Prototype Buildings 


\subsection{Building Form}

Table 3-2 lists the primary building form parameters for each prototype building. The building form parameters for each prototype were selected to best represent the typical construction of that building. The size (total area) of the prototype buildings range from $232 \mathrm{~m}^{2}\left(2500 \mathrm{ft}^{2}\right)$ to $46325 \mathrm{~m}^{2}$ (498 $640 \mathrm{ft}^{2}$ ), with the number of building stories ranging from 1 to 12 (excluding basements). The aspect ratio is defined as the total length of the building from east-to-west divided by the total length of the building from north-to-south. Given the building geometry of the primary and secondary schools, their aspect ratios are described as E-shaped. The aspect ratio of the outpatient healthcare prototype building is not defined (NA) given its complex geometry. The large hotel is the only prototype building with two aspect ratios: 3.8 and 5.1. This is due to differences in the dimensions of the building's first floor and its remaining five floors. The window-to-wall glazing fractions range from $0.7 \%$ to $40.0 \%$. For additional information on the building form specifications for each prototype building, refer to Thornton, Rosenberg et al. (2011) or the downloadable PNNL prototype scorecards (US Department of Energy (DOE) n.d.). 
Table 3-2 Building Form Parameters

\begin{tabular}{|c|c|c|c|c|c|c|}
\hline $\begin{array}{l}\text { Prototype } \\
\text { Building }\end{array}$ & $\begin{array}{c}\text { Floor } \\
\text { Area m² } \\
\left(\mathrm{ft}^{2}\right)\end{array}$ & $\begin{array}{l}\text { Number } \\
\text { of Floors }\end{array}$ & $\begin{array}{l}\text { Aspect } \\
\text { Ratio }\end{array}$ & $\begin{array}{l}\text { Floor-to-Ceiling } \\
\left.\text { Height } \mathrm{m}^{2} \text { ( } \mathrm{ft}^{2}\right)\end{array}$ & $\begin{array}{l}\text { Floor-to-Floor } \\
\text { Height } \mathrm{m}^{2}\left(\mathrm{ft}^{2}\right)\end{array}$ & $\begin{array}{l}\text { Glazing } \\
\text { Fraction }\end{array}$ \\
\hline Small Office & $\begin{array}{c}511 \\
(5500)\end{array}$ & 1 & 1.5 & 3.05 (10) & 3.05 (10) & $21 \%$ \\
\hline $\begin{array}{l}\text { Medium } \\
\text { Office }\end{array}$ & $\begin{array}{c}4982 \\
(53630)\end{array}$ & 3 & 1.5 & $2.74(9)$ & 3.96 (13) & $33 \%$ \\
\hline Large Office & $\begin{array}{c}46325 \\
(498640)\end{array}$ & $12^{(\mathrm{a})}$ & 1.5 & $2.74(9)$ & 3.96 (13) & $40 \%$ \\
\hline $\begin{array}{l}\text { Primary } \\
\text { School }\end{array}$ & $\begin{array}{c}6872 \\
(73970)\end{array}$ & 1 & E-Shape & 3.96 (13) & 3.96 (13) & $35 \%$ \\
\hline $\begin{array}{l}\text { Secondary } \\
\text { School }\end{array}$ & $\begin{array}{c}19594 \\
(210910)\end{array}$ & 2 & E-Shape & 3.96 (13) & $3.96(13)$ & $35 \%$ \\
\hline $\begin{array}{l}\text { Standalone } \\
\text { Retail }\end{array}$ & $\begin{array}{c}2294 \\
(24690)\end{array}$ & 1 & 1.3 & $6.10(20)$ & $6.10(20)$ & $7 \%$ \\
\hline Strip Mall & $\begin{array}{c}2090 \\
(22500)\end{array}$ & 1 & 4.0 & $5.18(17)$ & $5.18(17)$ & $11 \%$ \\
\hline $\begin{array}{l}\text { Quick-Service } \\
\text { Restaurant }\end{array}$ & $\begin{array}{c}232 \\
(2500)\end{array}$ & 1 & 1.0 & $3.05(10)$ & $3.05(10)$ & $14 \%$ \\
\hline $\begin{array}{l}\text { Full-Service } \\
\text { Restaurant }\end{array}$ & $\begin{array}{c}511 \\
(5500)\end{array}$ & 1 & 1.0 & $3.05(10)$ & $3.05(10)$ & $17 \%$ \\
\hline Small Hotel & $\begin{array}{c}4014 \\
(43210)\end{array}$ & 4 & 3.0 & $\begin{array}{c}3.35^{(\mathrm{a})} / 2.74 \\
(11 / 9)\end{array}$ & $\begin{array}{c}3.35^{(\mathrm{a})} / 2.74 \\
\left(11^{(\mathrm{c})} / 9\right)\end{array}$ & $12 \%$ \\
\hline Large Hotel & $\begin{array}{c}11345 \\
(122120)\end{array}$ & $6^{(\mathrm{b})}$ & $3.8^{(\mathrm{c})} / 5.1$ & $\begin{array}{c}3.96^{(\mathrm{a})} / 3.05 \\
(13 / 10)\end{array}$ & $\begin{array}{c}3.96^{(\mathrm{a})} / 3.05 \\
(13 / 10)\end{array}$ & $30 \%$ \\
\hline $\begin{array}{l}\text { Outpatient } \\
\text { Healthcare }\end{array}$ & $\begin{array}{c}3804 \\
(40950)\end{array}$ & 3 & NA & $3.05(10)$ & $3.05(10)$ & $20 \%$ \\
\hline Warehouse & $\begin{array}{c}4836 \\
(52050)\end{array}$ & 1 & 2.2 & 8.53 (28) & 8.53 (28) & $0.71 \%$ \\
\hline $\begin{array}{l}\text { Mid-Rise } \\
\text { Apartment }\end{array}$ & $\begin{array}{c}3135 \\
(33740)\end{array}$ & 4 & 2.7 & $3.05(10)$ & $3.05(10)$ & $20 \%$ \\
\hline $\begin{array}{l}\text { High-Rise } \\
\text { Apartment }\end{array}$ & $\begin{array}{c}7837 \\
(84360)\end{array}$ & 10 & 2.8 & $3.05(10)$ & $3.05(10)$ & $30 \%$ \\
\hline
\end{tabular}

\subsection{Building Fabric}

Table 3-3 lists the areas and construction types for the primary components of the building's envelope or fabric: roof, walls, foundation, and windows. Roof area ranges from $259 \mathrm{~m}^{2}$ (2786 $\mathrm{ft}^{2}$ ) to $11903 \mathrm{~m}^{2}\left(128120 \mathrm{ft}^{2}\right)$. Each prototype building adopts one of three roof types. The first type, Insulation Entirely Above Deck (IEAD), assumes continuous insulation above the roof deck. The second type, Attic \& Other, assumes that insulation is installed between the roof joists. In the case of the third and final type (Metal Building) the insulation is located between structural members.

Insulated wall area ranges from $153 \mathrm{~m}^{2}\left(1643 \mathrm{ft}^{2}\right)$ to $6954 \mathrm{~m}^{2}\left(78849 \mathrm{ft}^{2}\right)$. Each prototype building adopts one of four wall construction types as defined by ASHRAE 90.1-2004. The Wood Framed and Other Wall and Steel Framed Wall types are characterized as having framed walls with different thermal layers. The Mass Wall type uses continuous insulation, 
while the Metal Building Wall type has insulation compressed between structural components. Twelve of the prototype buildings are modeled with $10.2 \mathrm{~cm}$ (4.0 in) heavyweight concrete slabon-grade foundations. The warehouse also has a slab-on-grade foundation but modeled with a thickness of $20.3 \mathrm{~cm}$ (8.0 in). The large hotel and large office are modeled with basements. For additional information on the fabric specifications for each prototype, refer to Thornton, Rosenberg et al. (2011) or the downloadable PNNL prototype scorecards (US Department of Energy (DOE) n.d.).

Table 3-3 Building Fabric Specifications: Roof, Exterior Walls, and Foundation

\begin{tabular}{|c|c|c|c|c|c|c|}
\hline $\begin{array}{l}\text { Prototype } \\
\text { Building }\end{array}$ & $\begin{array}{c}\text { Roof Area } \\
\mathrm{m}^{2}\left(\mathrm{ft}^{2}\right)\end{array}$ & $\begin{array}{c}\text { Roof } \\
\text { Construction }\end{array}$ & $\begin{array}{l}\text { Wall Area } \\
\mathrm{m}^{2}\left(\mathrm{ft}^{2}\right)^{*}\end{array}$ & $\begin{array}{l}\text { Wall } \\
\text { Const. }\end{array}$ & $\begin{array}{l}\text { Foundation Floor } \\
\text { Area } \mathrm{m}^{2}\left(\mathrm{ft}^{2}\right)\end{array}$ & $\begin{array}{l}\text { Foundation } \\
\text { Floor Type }\end{array}$ \\
\hline Small Office & $\begin{array}{c}599 \\
(6443)\end{array}$ & Attic \& Other & $222(2388)$ & $\begin{array}{l}\text { Wood } \\
\text { Framed }\end{array}$ & $511(5500)$ & Slab \\
\hline $\begin{array}{l}\text { Medium } \\
\text { Office }\end{array}$ & $\begin{array}{c}1660 \\
(17867)\end{array}$ & IEAD & 1325 (14 263) & $\begin{array}{c}\text { Steel } \\
\text { Framed }\end{array}$ & 1661 (17 876) & Slab \\
\hline Large Office & $\begin{array}{c}3563 \\
(38354)\end{array}$ & IEAD & 6954 (74 849) & Mass & 3860 (41 553) & Basement \\
\hline $\begin{array}{l}\text { Primary } \\
\text { School }\end{array}$ & $\begin{array}{c}6875 \\
(73970)\end{array}$ & IEAD & 1633 (17 575) & $\begin{array}{c}\text { Steel } \\
\text { Framed }\end{array}$ & $6872(73$ 970) & Slab \\
\hline $\begin{array}{l}\text { Secondary } \\
\text { School }\end{array}$ & $\begin{array}{c}11903 \\
(128120)\end{array}$ & IEAD & 3879 (41 755) & $\begin{array}{c}\text { Steel } \\
\text { Framed }\end{array}$ & 9797 (105 455) & Slab \\
\hline $\begin{array}{l}\text { Standalone } \\
\text { Retail }\end{array}$ & $\begin{array}{c}2294 \\
(24690)\end{array}$ & IEAD & 1093 (11 766) & Mass & 2294 (24 690) & Slab \\
\hline Strip Mall & $\begin{array}{c}2090 \\
(22500)\end{array}$ & IEAD & 1060 (11 408) & $\begin{array}{c}\text { Steel } \\
\text { Framed }\end{array}$ & $2090(22500)$ & Slab \\
\hline $\begin{array}{l}\text { Quick-Service } \\
\text { Restaurant }\end{array}$ & $\begin{array}{c}259 \\
(2786)\end{array}$ & Attic \& Other & 153 (1643) & $\begin{array}{l}\text { Wood } \\
\text { Framed }\end{array}$ & $232(2500)$ & Slab \\
\hline $\begin{array}{l}\text { Full-Service } \\
\text { Restaurant }\end{array}$ & $\begin{array}{c}569 \\
(6130)\end{array}$ & Attic \& Other & $229(2460)$ & $\begin{array}{c}\text { Steel } \\
\text { Framed }\end{array}$ & $511(5500)$ & Slab \\
\hline Small Hotel & $\begin{array}{c}1003 \\
(10802)\end{array}$ & IEAD & 1510 (16 258) & $\begin{array}{c}\text { Steel } \\
\text { Framed }\end{array}$ & 1004 (10 802) & Slab \\
\hline Large Hotel & $\begin{array}{c}1979 \\
(21300)\end{array}$ & IEAD & 2812 (30 265) & Mass & 1979 (21 300) & Basement \\
\hline $\begin{array}{l}\text { Outpatient } \\
\text { Healthcare }\end{array}$ & $\begin{array}{c}1373 \\
(14782)\end{array}$ & IEAD & 1245 (13 402) & $\begin{array}{c}\text { Steel } \\
\text { Frame }\end{array}$ & $1268(13650)$ & Slab \\
\hline Warehouse & $\begin{array}{c}4598 \\
(49495)\end{array}$ & $\begin{array}{c}\text { Metal } \\
\text { Building Roof }\end{array}$ & 1510 (16 258) & $\begin{array}{c}\text { Metal } \\
\text { Building }\end{array}$ & 4836 (52 050) & Slab \\
\hline $\begin{array}{l}\text { Mid-Rise } \\
\text { Apartment }\end{array}$ & $\begin{array}{c}784 \\
(8435)\end{array}$ & IEAD & 1235 (13 295) & $\begin{array}{l}\text { Steel } \\
\text { Frame }\end{array}$ & 784 (8435) & Slab \\
\hline $\begin{array}{l}\text { High-Rise } \\
\text { Apartment }\end{array}$ & $\begin{array}{c}784 \\
(8435)\end{array}$ & IEAD & 2705 (29 112) & $\begin{array}{l}\text { Steel } \\
\text { Frame }\end{array}$ & 784 (8435) & Slab \\
\hline
\end{tabular}

ASHRAE standards provide fenestration requirements based on fenestration type. In the case of windows, ASHRAE 90.1-2004 defines two types of windows: fixed and operable. Later editions of the standard more explicitly define window types, classifying them as either nonmetal, metal curtain wall/storefront, metal entrance door and all other windows. Table 3-4 describes the types of exterior windows associated with each building type based on weighting factors (\%). For example, in the case of the small office, Standard 90.1-2004 requires $95 \%$ of its windows to be fixed and $5 \%$ operable. ASHRAE 90.1-2007, -2010, and -2013 requires more than half of its windows to be of "Metal Curtain Wall/Storefront" type. The information in Table 3-4 can be 
found by referring to Thornton, Rosenberg et al. (2011) or the downloadable PNNL prototype scorecards (US Department of Energy (DOE) n.d.).

Table 3-4 Building Fabric Specifications: Exterior Windows

\begin{tabular}{|lccccc|}
\hline \multicolumn{1}{|c}{ Prototype Building } & Fixed & Operable & Nonmetal & $\begin{array}{c}\text { Metal Curtain } \\
\text { Wall/Storefront }\end{array}$ & $\begin{array}{c}\text { Metal, All Other } \\
\text { Small Office }\end{array}$ \\
\hline Medium Office & $95 \%$ & $5 \%$ & $2 \%$ & $62 \%$ & $36 \%$ \\
\hline Large Office & $95 \%$ & $5 \%$ & $2 \%$ & $62 \%$ & $36 \%$ \\
\hline Primary School & $65 \%$ & $0 \%$ & $2 \%$ & $62 \%$ & $36 \%$ \\
\hline Secondary School & $65 \%$ & $35 \%$ & $22 \%$ & $45 \%$ & $34 \%$ \\
\hline Standalone Retail & $98 \%$ & $2 \%$ & $10 \%$ & $45 \%$ & $34 \%$ \\
\hline Strip Mall & $98 \%$ & $2 \%$ & $10 \%$ & $62 \%$ & $28 \%$ \\
\hline $\begin{array}{l}\text { Quick-Service } \\
\text { Restaurant }\end{array}$ & $98 \%$ & $2 \%$ & $10 \%$ & $62 \%$ & $28 \%$ \\
\hline Full-Service Restaurant & $98 \%$ & $2 \%$ & $10 \%$ & $62 \%$ & $28 \%$ \\
\hline Small Hotel & $78 \%$ & $22 \%$ & $11 \%$ & $21 \%$ & $68 \%$ \\
\hline Large Hotel & $78 \%$ & $22 \%$ & $11 \%$ & $21 \%$ & $68 \%$ \\
\hline Outpatient Healthcare & $88 \%$ & $12 \%$ & $9 \%$ & $60 \%$ & $31 \%$ \\
\hline Warehouse & $95 \%$ & $5 \%$ & $2 \%$ & $10 \%$ & $88 \%$ \\
\hline Mid-Rise Apartment & $42 \%$ & $58 \%$ & $32 \%$ & $5 \%$ & $63 \%$ \\
\hline High-Rise Apartment & $42 \%$ & $58 \%$ & $32 \%$ & $5 \%$ & $63 \%$ \\
\hline
\end{tabular}

\subsection{Building Occupancy}

Information on the total number of assumed occupants assumed for each prototype are listed in Table 3-5. Occupancy densities provide some insight into the individual contributions of each occupant to building energy loads. Occupancy rates are consistent with DOE reference buildings and are based on ASHRAE Advanced Energy Design Guide (AEDG) studies and default rates given by ASHRAE 62.1-2004 (ANSI/ASHRAE 2004, ASHRAE/AIA/IESNA 2009). The maximum number of occupants varies between 5 occupants and 6096 occupants, with average $\mathrm{m}^{2}\left(\mathrm{ft}^{2}\right)$ per occupant ranging from $2.32 \mathrm{~m}^{2}\left(25 \mathrm{ft}^{2}\right)$ to $33.29 \mathrm{~m}^{2}\left(358.36 \mathrm{ft}^{2}\right)$. The information in Table 3-5 can be found by referring to Thornton, Rosenberg et al. (2011) or the downloadable PNNL prototype scorecards (US Department of Energy (DOE) n.d.). 
Table 3-5 Building Occupancy Rates

\begin{tabular}{|ccc|}
\hline Prototype Building & $\begin{array}{c}\text { Occupant Densities } \\
\text { (\# of people) }\end{array}$ & $\begin{array}{c}\text { Average } \mathbf{~ m}^{\mathbf{2}} \mathbf{( f t}^{\mathbf{2}} \text { ) Per } \\
\text { Occupant }\end{array}$ \\
\hline Small Office & 31 & $16.63(179)$ \\
\hline Medium Office & 268 & $18.58(200)$ \\
\hline Large Office & 2493 & $18.10(194.7)$ \\
\hline Primary School & 1477 & $3.96(42.62)$ \\
\hline Secondary School & 6096 & $2.98(32.09)$ \\
\hline Standalone Retail & 371 & $6.19(66.6)$ \\
\hline Strip Mall & 180 & $11.61(125)$ \\
\hline Quick-Service Restaurant & 94 & $9.92(106.8)$ \\
\hline Full-Service Restaurant & 287 & $6.02(64.8)$ \\
\hline Small Hotel & 259 & $16.13(173.61)$ \\
\hline Large Hotel & 1494 & $31.19(335.69)$ \\
\hline Outpatient Healthcare & 419 & $5.52(59.43)$ \\
\hline Warehouse & 5 & $2.32(25)$ \\
\hline Mid-Rise Apartment & 79 & $33.29(358.36)$ \\
\hline High-Rise Apartment & 199 & $32.40(348.73)$ \\
\hline
\end{tabular}

\subsection{Service Water Heating}

Table 3-6 provides the characteristics of the modeled service water heating (SWH) systems included in each of the prototype buildings. The SWH system provides the hot water necessary to satisfy numerous building activities (e.g. cooking, laundry). Some of the prototype models (i.e. Primary School and Large Hotel) use both natural gas and electricity to heat water above initial temperatures. The primary and secondary school models include a main natural gas water heater for the buildings' primary hot water needs, and a second electric water heater for the dishwasher booster. The large hotel uses natural gas water heaters (2) for primary hot water demands (2271.3 liters) and the laundry (1135.6 liters), while an electric water heater is used for the dishwasher booster (22.7 liters).

Like the primary school, secondary school and large hotel prototype buildings, the full-service restaurant, strip mall, small hotel, and mid-rise apartments are modeled using more than one hot water tank. The full-service restaurant includes two natural gas water heaters: one serving as the primary system and the other serving as a dishwasher booster. The strip mall prototype includes electric water heaters for 7 of the 10 store units. The small hotel prototype model includes a natural gas water heater tank serving as the primary system, and another gas tank for the laundry. The mid-rise apartment includes an electric hot water heating tank for each of its 23 apartment units. The service water heating efficiency requirements - indicated by thermal efficiency ratio - remains constant across ASHRAE 90.1 editions. Additional information on the SWH equipment for each of the prototype models can be found by referring to Thornton, Rosenberg et al. (2011) or the downloadable PNNL prototype scorecards (US Department of Energy (DOE) n.d.). 
Table 3-6 Service Water Heating Equipment by Building Type

\begin{tabular}{|c|c|c|c|}
\hline Prototype Building & Energy Source $^{(a)}$ & $\begin{array}{c}\text { Number of Systems } \\
\text { (Modeled) }\end{array}$ & $\begin{array}{l}\text { Storage Capacity } \\
\text { liters (gallons) }^{(\mathbf{b})}\end{array}$ \\
\hline Small Office & Electric & 1 & $151.4(40)$ \\
\hline Medium Office & Natural Gas & 1 & $387.5(100)$ \\
\hline Large Office & Natural Gas & 1 & $1135.6(300)$ \\
\hline Primary School & Natural Gas/Electric & 2 & $757.1(200) / 22.7(6)$ \\
\hline Secondary School & Natural Gas/Electric & 2 & $2271.3(600) / 22.7(6)$ \\
\hline Standalone Retail & Natural Gas & 1 & $151.4(40)$ \\
\hline Strip Mall & Electric & 7 & $151.4(40)$ \\
\hline $\begin{array}{c}\text { Quick-Service } \\
\text { Restaurant }\end{array}$ & Natural Gas & 1 & $387.5(100)$ \\
\hline $\begin{array}{l}\text { Full-Service } \\
\text { Restaurant }\end{array}$ & Natural Gas & 2 & $757.1(200) / 22.7(6)$ \\
\hline Small Hotel & Natural Gas & 2 & $1135.6(300) / 757.1(200)$ \\
\hline Large Hotel & Natural Gas/Electric & 3 & $2271.3(600) / 1135.6(300) / 22.7(6)$ \\
\hline $\begin{array}{l}\text { Outpatient } \\
\text { Healthcare }\end{array}$ & Natural Gas & 1 & $757.1(200)$ \\
\hline Warehouse & Electric & 1 & 75.7 (20) \\
\hline Mid-Rise Apartment & Electric & 31 & $189.3(50)$ \\
\hline $\begin{array}{l}\text { High-Rise } \\
\text { Apartment }\end{array}$ & Natural Gas & 1 & $2271.3(600)$ \\
\hline
\end{tabular}

(a) The first energy source is used to heat the water of the primary or main system(s). The secondary energy source heats serves as the secondary energy source.

(b) The first value is the capacity for the primary SHW storage tank (s). The second value is the capacity of the second tank. Only in the case of the Large Hotel is there a third value for a third tank.

\subsection{HVAC Systems}

HVAC system requirements outlined by ASHRAE Standard 90.1 are dependent on and vary based on the type of system(s) included in the building. Standard requirements for other HVACrelated characteristics such as equipment efficiency are also dependent on system types. The following sections discuss the HVAC system(s) associated with each prototype building, efficiency requirements for individual system components, as well as requirements for outdoor air ventilation.

\subsubsection{HVAC System Types and Equipment Capacities}

Table 3-7 lists the heating and cooling equipment of both the primary and secondary HVAC systems associated with the PNNL prototype buildings. The primary systems are generally responsible for conditioning thermal zones with similar operating characteristics, whereas secondary systems satisfy heating and cooling loads for thermal zones that have operating needs differing from zones conditioned by the primary system. Majority of the prototype buildings' primary HVAC systems are constant air volume (CAV) unitary packaged systems, while some others use boilers, chillers, and/or fluid coolers and air distribution systems to condition zones. Given their large size (floor area) and high occupant density, the large office, primary school, secondary school and large hotel each include equipment for a secondary HVAC system. 
Table 3-7 HVAC Equipment by Building Type

\begin{tabular}{|c|c|c|c|c|}
\hline $\begin{array}{l}\text { Prototype } \\
\text { Building }\end{array}$ & Heating & Cooling & Primary System & $\begin{array}{l}\text { Secondary } \\
\text { System }\end{array}$ \\
\hline Small Office & Air-Source Heat Pump & Air-Source Heat Pump & Packaged CAV ${ }^{(a)}$ & No \\
\hline Medium Office & Gas Furnace & Unitary $\mathrm{DX}^{(\mathrm{b})}$ & $\begin{array}{l}\text { Packaged VAV } \\
\text { w/Reheat }\end{array}$ & No \\
\hline Large Office & Boiler & $\begin{array}{c}\text { Centrifugal Chiller/Cooling } \\
\text { Tower }\end{array}$ & VAV w/Reheat & WSHP \\
\hline $\begin{array}{c}\text { Primary } \\
\text { School }\end{array}$ & Gas Furnace/Boiler & Unitary DX & Packaged CAV & $\begin{array}{c}\text { VAV } \\
\text { w/Reheat }\end{array}$ \\
\hline $\begin{array}{l}\text { Secondary } \\
\text { School }\end{array}$ & Gas Furnace/Gas Boiler & Unitary DX/Air-cooled Chiller & Packaged CAV & $\begin{array}{c}\text { VAV } \\
\text { w/Reheat }\end{array}$ \\
\hline $\begin{array}{l}\text { Standalone } \\
\text { Retail }\end{array}$ & $\begin{array}{c}\text { Gas Furnace/ } \\
\text { Standalone Furnace }\end{array}$ & Unitary DX & Packaged CAV & No \\
\hline Strip Mall & Gas Furnace & Unitary DX & Packaged CAV & No \\
\hline $\begin{array}{l}\text { Quick-Service } \\
\text { Restaurant }\end{array}$ & Gas Furnace & Unitary DX & Packaged CAV & No \\
\hline $\begin{array}{c}\text { Full-Service } \\
\text { Restaurant }\end{array}$ & Gas Furnace & Unitary DX & Packaged CAV & No \\
\hline Small Hotel & $\begin{array}{c}\text { Electricity/Gas/Cabinet } \\
\text { Heater }\end{array}$ & DX & PTAC & Split System \\
\hline Large Hotel & Boiler & Air-cooled Chiller & Fan-Coil Units & $\begin{array}{c}\text { VAV } \\
\text { w/Reheat }\end{array}$ \\
\hline $\begin{array}{l}\text { Outpatient } \\
\text { Healthcare }\end{array}$ & Boiler & Unitary DX & $\begin{array}{l}\text { Packaged VAV } \\
\text { w/Reheat }\end{array}$ & No \\
\hline Warehouse & $\begin{array}{c}\text { Gas Furnace/Gas Unit } \\
\text { Heater }\end{array}$ & Unitary DX & Packaged CAV & No \\
\hline $\begin{array}{l}\text { Mid-Rise } \\
\text { Apartment }\end{array}$ & Gas & DX & Split System & No \\
\hline $\begin{array}{l}\text { High-Rise } \\
\text { Apartment }\end{array}$ & Boiler & Fluid Cooler & $\begin{array}{c}\text { Water-Source Heat } \\
\text { Pump }\end{array}$ & No \\
\hline \multicolumn{5}{|c|}{$\begin{array}{l}\text { (a) CAV - Constant Air Volume } \\
\text { (a) DX - Direct Expansion } \\
\text { (a) VAV - Variable Air Volume }\end{array}$} \\
\hline
\end{tabular}

Although Standard 90.1 provides recommendations on system types for different primary building activities, they are often inconsistent with typical systems found in existing buildings. Prototype HVAC system types are consistent with those included in the DOE Reference Buildings, which are largely based on an analysis of the 2003 CBECS dataset by Winiarski et al. (2006).

External climate conditions, ventilation rates, and internal loads are all factors impacting the HVAC equipment capacities for each prototype building. Given that these factors vary with ASHRAE Standard edition, equipment capacities are also likely to vary across editions. Equipment capacities are generally determined using a "autosizing" approach, where HVAC equipment is properly sized such that indoor comfort is maintained and HVAC operational energy use is minimized. Building energy simulation models such as EnergyPlus $\left(\mathrm{E}^{+}\right)$ incorporate autosizing methods to help approximate the appropriate HVAC equipment capacities 
for a given building design. The autosizing of HVAC equipment for the prototype buildings will later be discussed in Chapter 4 .

\subsubsection{HVAC Equipment Efficiencies}

The minimum efficiency requirements for HVAC equipment included in the prototype building models are listed in Table 3-8 (heating-related equipment) and Table 3-9 (cooling-related equipment). The values listed in both tables come directly from the prototype buildings modeled in OpenStudio. Efficiencies for each HVAC system component are assigned using methodologies described in ASHRAE 90.1, where minimum efficiencies are set based on system capacity. They are defined in terms of a coefficient of performance (COP), energy efficiency ratio (EER), seasonal energy efficiency ratio (SEER), water flow rating at rating conditions divided by the motor nameplate power (gpm/hp), or a boiler and furnace efficiency. The minimum efficiencies for both the heating- and cooling-related system equipment either increases or remains constant in later editions. In the case of heating-related equipment, system efficiencies change for boilers and heat pumps between 90.1-2004 and 90.1-2007 while the other system efficiencies remain constant. Cooling-related equipment efficiency realizes more incremental increases across editions, particularly between 90.1-2007 and 90.1-2010. For more information on the efficiencies assigned to the PNNL prototype building model HVAC equipment, please refer to Thornton, Rosenberg et al. (2011) or the downloadable PNNL prototype scorecards (US Department of Energy (DOE) n.d.). 
Table 3-8 HVAC Equipment Minimum Efficiencies - Heating

\begin{tabular}{|c|c|c|c|c|c|c|}
\hline $\begin{array}{l}\text { Prototype } \\
\text { Building }\end{array}$ & Heating & 90.1-2004 & 90.1-2007 & 90.1-2010 & 90.1-2013 & $90.1-2016$ \\
\hline Small Office & $\begin{array}{l}\text { Air-Source Heat } \\
\text { Pump }\end{array}$ & 6.6 $\mathrm{HSPF}^{(\mathrm{a})}$ & 7.7 HSPF & 7.7 HSPF & 7.7 HSPF & 7.7 HSPF \\
\hline Medium Office & Gas Furnace & $0.80 \mathrm{Eff}^{(\mathrm{b})}$ & $0.80 \mathrm{Eff}$ & $0.80 \mathrm{Eff}$ & $0.80 \mathrm{Eff}$ & 0.80 Eff \\
\hline Large Office & Boiler & $0.75 \mathrm{E}_{\mathrm{t}}^{(\mathrm{c})}$ & $0.80 \mathrm{E}_{\mathrm{t}}$ & $0.80 \mathrm{E}_{\mathrm{t}}$ & $0.80 \mathrm{E}_{\mathrm{t}}$ & $0.80 \mathrm{E}_{\mathrm{t}}$ \\
\hline $\begin{array}{c}\text { Primary } \\
\text { School }\end{array}$ & Gas Furnace/Boiler & $\begin{array}{c}0.80 \\
\text { Eff/0.75 } \mathrm{E}_{\mathrm{t}}\end{array}$ & $\begin{array}{c}0.80 \\
\text { Eff/0.80 } \mathrm{E}_{\mathrm{t}}\end{array}$ & $\begin{array}{c}0.80 \\
\text { Eff/0.80 } \mathrm{E}_{\mathrm{t}}\end{array}$ & $\begin{array}{c}0.80 \\
\text { Eff/0.80 } \mathrm{E}_{\mathrm{t}}\end{array}$ & $\begin{array}{c}0.80 \\
\text { Eff/0.80 } E_{t}\end{array}$ \\
\hline $\begin{array}{l}\text { Secondary } \\
\text { School }\end{array}$ & $\begin{array}{c}\text { Gas Furnace/Gas } \\
\text { Boiler }\end{array}$ & $\begin{array}{c}0.80 \\
\text { Eff/0.75 } \mathrm{E}_{\mathrm{t}}\end{array}$ & $\begin{array}{c}0.80 \\
\text { Eff/0.80 } \mathrm{E}_{\mathrm{t}}\end{array}$ & $\begin{array}{c}0.80 \\
\text { Eff/0.80 } \mathrm{E}_{\mathrm{t}}\end{array}$ & $\begin{array}{c}0.80 \\
\text { Eff/0.80 } \mathrm{E}_{\mathrm{t}}\end{array}$ & $\begin{array}{c}0.80 \\
\text { Eff/0.80 } E_{t}\end{array}$ \\
\hline $\begin{array}{l}\text { Standalone } \\
\text { Retail }\end{array}$ & $\begin{array}{c}\text { Gas Furnace/ } \\
\text { Standalone Furnace }\end{array}$ & 0.80 Eff & $0.80 \mathrm{Eff}$ & $0.80 \mathrm{Eff}$ & $0.80 \mathrm{Eff}$ & $0.80 \mathrm{Eff}$ \\
\hline Strip Mall & Gas Furnace & $0.80 \mathrm{Eff}$ & 0.80 Eff & $0.80 \mathrm{Eff}$ & 0.80 Eff & 0.80 Eff \\
\hline $\begin{array}{l}\text { Quick-Service } \\
\text { Restaurant }\end{array}$ & Gas Furn & 0.8 & 0.80 & 0.8 & 0.8 & 0.80 Eff \\
\hline $\begin{array}{l}\text { Full-Service } \\
\text { Restaurant }\end{array}$ & Gas Furnace & $0.80 \mathrm{Eff}$ & $0.80 \mathrm{Eff}$ & $0.80 \mathrm{Eff}$ & $0.80 \mathrm{Eff}$ & 0.80 Eff \\
\hline Small Hotel & $\begin{array}{l}\text { Electricity/ Gas/ } \\
\text { Cabinet Heater }\end{array}$ & $\begin{array}{l}1.0 \text { Eff/0.8 } \\
\text { Eff* }\end{array}$ & $\begin{array}{c}1.0 \mathrm{Eff} / 0.8 \\
\mathrm{Eff}^{*}\end{array}$ & $\begin{array}{c}1.0 \mathrm{Eff} / 0.8 \\
\mathrm{Eff}^{*}\end{array}$ & $\begin{array}{c}1.0 \mathrm{Eff} / 0.8 \\
\mathrm{Eff}^{*}\end{array}$ & $\begin{array}{l}1.0 \mathrm{Eff} / 0.8 \\
\text { Eff* }\end{array}$ \\
\hline Large Hotel & Boiler & $0.75 E_{t}$ & $0.80 \mathrm{E}_{\mathrm{t}}$ & $0.80 \mathrm{E}_{\mathrm{t}}$ & $0.80 \mathrm{E}_{\mathrm{t}}$ & $0.80 \mathrm{E}_{\mathrm{t}}$ \\
\hline $\begin{array}{l}\text { Outpatient } \\
\text { Healthcare }\end{array}$ & & $\begin{array}{c}0.80 \\
\text { AFUE }^{(\mathrm{d})}\end{array}$ & 0.80 AFUE & 0.80 AFUE & 0.80 AFUE & $0.80 \mathrm{AFUE}$ \\
\hline Warehouse & $\begin{array}{c}\text { Gas Furnace/Gas } \\
\text { Unit Heater }\end{array}$ & $0.80 \mathrm{Eff}$ & $0.80 \mathrm{Eff}$ & $0.80 \mathrm{Eff}$ & $0.80 \mathrm{Eff}$ & 0.80 Eff \\
\hline $\begin{array}{l}\text { Mid-Rise } \\
\text { Apartment }\end{array}$ & Gas & fff & 0.80 Eff & $0.80 \mathrm{Eff}$ & $0.80 \mathrm{Eff}$ & $0.80 \mathrm{Eff}$ \\
\hline $\begin{array}{l}\text { High-Rise } \\
\text { Apartment }\end{array}$ & Boiler & 0.80 AFUE & 0.80 AFUE & 0.80 AFUE & 0.80 AFUE & 0.80 AFUE \\
\hline \multicolumn{7}{|c|}{$\begin{array}{l}\text { (a) HSPF - Heating Seasonal Performance Factor } \\
\text { (b) Eff - Efficiency } \\
\text { (c) Et - Thermal Efficiency } \\
\text { (d) AFUE - Annual Fuel Utilization Efficiency } \\
\text { * AFUE - The } 1.0 \text { efficiency corresponds to the heating efficiency of both the PTAC and } \\
\text { cabinet heater }\end{array}$} \\
\hline
\end{tabular}


Table 3-9 HVAC Equipment Minimum Efficiencies - Cooling

\begin{tabular}{|c|c|c|c|c|c|c|}
\hline $\begin{array}{c}\text { Prototype } \\
\text { Building }\end{array}$ & Cooling & 90.1-2004 & 90.1-2007 & 90.1-2010 & 90.1-2013 & 90.1-2016 \\
\hline Small Office & $\begin{array}{c}\text { Air-Source Heat } \\
\text { Pump }\end{array}$ & 9.7 SEER ${ }^{(a)}$ & $\begin{array}{l}13.0 \\
\text { SEER }\end{array}$ & $\begin{array}{l}13.0 \\
\text { SEER }\end{array}$ & $\begin{array}{c}13.0 \\
\text { SEER }\end{array}$ & $\begin{array}{l}13.0 \\
\text { SEER }\end{array}$ \\
\hline $\begin{array}{c}\text { Medium } \\
\text { Office }\end{array}$ & Unitary DX & $9.3 \mathrm{EER}^{(\mathrm{b})}$ & 9.3 EER & 9.8 EER & 9.8 EER & 9.8 EER \\
\hline Large Office & $\begin{array}{c}\text { Centrifugal } \\
\text { Chiller/Cooling } \\
\text { Tower }\end{array}$ & $\begin{array}{l}6.1 \mathrm{COP}^{(\mathrm{c})} / \\
38.2 \\
\mathrm{gpm} / \mathrm{hp}^{(\mathrm{d})}\end{array}$ & $\begin{array}{l}\text { 6.2 COP/ } \\
38.2 \\
\mathrm{gpm} / \mathrm{hp}\end{array}$ & $\begin{array}{c}6.2 \mathrm{COP} / \\
14.0 \\
\mathrm{gpm} / \mathrm{hp}\end{array}$ & $\begin{array}{c}6.3 \mathrm{COP} / \\
14.0 \\
\mathrm{gpm} / \mathrm{hp}\end{array}$ & $\begin{array}{c}\text { 6.3 COP/ } \\
14.0 \\
\mathrm{gpm} / \mathrm{hp}\end{array}$ \\
\hline $\begin{array}{c}\text { Primary } \\
\text { School }\end{array}$ & Unitary DX & 10.1 EER & 10.1 EER & 11.0 EER & 11.0 EER & 11.0 EER \\
\hline $\begin{array}{l}\text { Secondary } \\
\text { School }\end{array}$ & $\begin{array}{l}\text { Unitary DX/Air- } \\
\text { cooled Chiller }\end{array}$ & $\begin{array}{l}\text { 10.1 EER/ } \\
2.8 \mathrm{COP}\end{array}$ & $\begin{array}{c}10.1 \\
\text { EER/ } \\
2.8 \mathrm{COP}\end{array}$ & $\begin{array}{c}11.0 \\
\text { EER/ } \\
2.8 \mathrm{COP}\end{array}$ & $\begin{array}{c}11.0 \\
\text { EER/ } \\
3.0 \mathrm{COP}\end{array}$ & $\begin{array}{c}11.0 \\
\text { EER/ } \\
3.0 \mathrm{COP}\end{array}$ \\
\hline $\begin{array}{c}\text { Standalone } \\
\text { Retail }\end{array}$ & Unitary DX & 10.1 EER & 10.1 EER & 11.0 EER & 11.0 EER & 11.0 EER \\
\hline Strip Mall & Unitary DX & 10.1 EER & 10.1 EER & 11.0 EER & 11.0 EER & 11.0 EER \\
\hline $\begin{array}{c}\text { Quick-Service } \\
\text { Restaurant }\end{array}$ & Unitary DX & $\begin{array}{l}\text { 9.5 EER/ } \\
\text { 9.7 EER }\end{array}$ & $\begin{array}{l}\text { 9.5 EER/ } \\
10.1 \mathrm{EER}\end{array}$ & $\begin{array}{c}10.8 \\
\text { EER/ } \\
11.0 \mathrm{EER}\end{array}$ & $\begin{array}{c}10.8 \\
\text { EER/ } \\
11.0 \mathrm{EER}\end{array}$ & $\begin{array}{c}10.8 \\
\text { EER/ } \\
11.0 \text { EER }\end{array}$ \\
\hline $\begin{array}{l}\text { Full-Service } \\
\text { Restaurant }\end{array}$ & Unitary DX & $\begin{array}{l}\text { 9.3 EER/ } \\
\text { 9.5 EER }\end{array}$ & $\begin{array}{l}\text { 9.3 EER/ } \\
10.1 \mathrm{EER}\end{array}$ & $\begin{array}{l}\text { 9.8 EER/ } \\
11.0 \mathrm{EER}\end{array}$ & $\begin{array}{l}\text { 9.8 EER/ } \\
11.0 \mathrm{EER}\end{array}$ & $\begin{array}{l}\text { 9.8 EER/ } \\
11.0 \mathrm{EER}\end{array}$ \\
\hline Small Hotel & $\mathrm{DX}$ & $\begin{array}{l}9.3-11.0 \\
\text { EER/ } \\
9.7-10.1 \\
\text { SEER }\end{array}$ & $\begin{array}{l}9.3-11.0 \\
\text { EER/ } \\
10.1-13.0 \\
\text { SEER }\end{array}$ & $\begin{array}{l}9.3-11.7 \\
\text { EER/ } \\
11.0-13.0 \\
\text { SEER }\end{array}$ & $\begin{array}{l}9.5-11.9 \\
\text { EER/ } \\
14.0 \\
\text { SEER }\end{array}$ & $\begin{array}{l}\text { 9.5-11.9 } \\
\text { EER/ } \\
14.0 \\
\text { SEER }\end{array}$ \\
\hline Large Hotel & Air-cooled Chiller & $2.8 \mathrm{COP}$ & $2.8 \mathrm{COP}$ & $2.8 \mathrm{COP}$ & $3.0 \mathrm{COP}$ & $3.0 \mathrm{COP}$ \\
\hline $\begin{array}{l}\text { Outpatient } \\
\text { Healthcare }\end{array}$ & Unitary DX & $\begin{array}{l}9.5 \mathrm{EER} / \\
9.0 \mathrm{EER}\end{array}$ & $\begin{array}{l}\text { 9.5 EER/ } \\
\text { 9.3 EER }\end{array}$ & $\begin{array}{c}10.0 \\
\text { EER/ } \\
9.8 \mathrm{EER}\end{array}$ & $\begin{array}{c}10.0 \\
\mathrm{EER} / \\
9.8 \mathrm{EER}\end{array}$ & $\begin{array}{c}10.0 \\
\text { EER/ } \\
9.8 \mathrm{EER}\end{array}$ \\
\hline Warehouse & Unitary DX & $\begin{array}{l}\text { 9.7 SEER/ } \\
\text { 9.5 SEER }\end{array}$ & $\begin{array}{l}10.1 \\
\text { SEER/ } \\
9.5 \mathrm{SEER}\end{array}$ & $\begin{array}{l}11.0 \\
\text { SEER/ } \\
10.8 \\
\text { SEER }\end{array}$ & $\begin{array}{l}11.0 \\
\text { SEER/ } \\
10.8 \\
\text { SEER }\end{array}$ & $\begin{array}{l}11.0 \\
\text { SEER/ } \\
10.8 \\
\text { SEER }\end{array}$ \\
\hline $\begin{array}{l}\text { Mid-Rise } \\
\text { Apartment }\end{array}$ & $\mathrm{DX}$ & 9.7 SEER & $\begin{array}{c}13.0 \\
\text { SEER }\end{array}$ & $\begin{array}{c}13.0 \\
\text { SEER }\end{array}$ & $\begin{array}{c}14.0 \\
\text { SEER }\end{array}$ & $\begin{array}{l}14.0 \\
\text { SEER }\end{array}$ \\
\hline $\begin{array}{l}\text { High-Rise } \\
\text { Apartment }\end{array}$ & Fluid Cooler & $\begin{array}{c}38.2 \\
\text { gpm/hp }\end{array}$ & $\begin{array}{c}38.2 \\
\text { gpm/hp }\end{array}$ & $\begin{array}{c}14.0 \\
\text { gpm/hp }\end{array}$ & $\begin{array}{c}14.0 \\
\text { gpm/hp }\end{array}$ & $\begin{array}{c}14.0 \\
\text { gpm/hp }\end{array}$ \\
\hline \multicolumn{7}{|c|}{$\begin{array}{l}\text { (a) SEER - Seasonal Energy Efficiency Ratio } \\
\text { (b) EER - Energy Efficiency Ratio } \\
\text { (c) COP - Coefficient of Performance } \\
\text { (d) gpm/hp - water flow rating divided by the motor nameplate power }\end{array}$} \\
\hline
\end{tabular}

\subsubsection{Ventilation}

The minimum outdoor air (OA) ventilation requirements for each prototype building (expressed in terms of total cubic feet per minute $(\mathrm{cfm})$ and $\left.\mathrm{cfm} / \mathrm{ft}^{2}\right)^{5}$ are listed in Table 3-10. Ventilation requirements are inconsistent across 90.1 standards due to alternative versions of ASHRAE

\footnotetext{
${ }^{5}$ SI unit conversions for $\mathrm{cfm}$ and $\mathrm{cfm} / \mathrm{ft}^{2}$ are liters per second $(\mathrm{L} / \mathrm{s})$ and liters per second $\mathrm{m}^{2}\left((\mathrm{~L} / \mathrm{s}) / \mathrm{m}^{2}\right)$.
} 
Standard 62.1 regulating outdoor air requirements for each standard edition. The oldest standard edition referenced, 90.1-2004, follows ASHRAE 62.1-1999 for its air flow requirements, while standards 90.1-2007, 90.1-2010, 90.1-2013, and 90.1-2016 each adhere to 62.1-2004, 62.1-2007, 62.1-2010, and 62.1-2013 respectively, for their reference ventilation standards. Requirements are identical across the latter three standards given that ASHRAE 62.1-2004, -2007, and -2010 have similar minimum outdoor air rates for each prototype building. Ventilation requirements in Standard 62.1 are expressed based on space use type in one of two ways: (1) per person or (2) per unit of floor area. Requirements listed in Table 3-10 are the total outdoor air ventilation rates and are found by summing across all zone-level ventilation rates for a given prototype building. For more information on how outdoor air ventilation rates are incorporated into each prototype building, as well as how ventilation rates are calculated based on distribution system type, please refer to Thornton, Rosenberg et al. (2011). Additional information on ventilation rates for each prototype model can be found using the downloadable PNNL scorecards (US Department of Energy (DOE) n.d.). 
Table 3-10 Outdoor Ventilation Rates

\begin{tabular}{|c|c|c|c|c|c|c|c|}
\hline & \multirow[t]{2}{*}{$\begin{array}{l}\text { Prototype } \\
\text { Building }\end{array}$} & \multirow{2}{*}{$\begin{array}{c}\text { Floor } \\
\text { Area } \\
\mathbf{m}^{2}\left(\mathbf{f t}^{2}\right)\end{array}$} & \multirow{2}{*}{$\begin{array}{c}\text { Total } \\
\text { Occupants } \\
\text { (based on } \\
\text { 62.1-2004)* }\end{array}$} & \multicolumn{2}{|c|}{$\begin{array}{l}\text { Total OA Ventilation cfm } \\
\left(\mathbf{f t}^{3} / \mathrm{min}\right)^{* *}\end{array}$} & \multicolumn{2}{|c|}{ Total OA Ventilation } \\
\hline & & & & 2004 & $\begin{array}{c}2007,-2010,- \\
2013,-2016\end{array}$ & -2004 & $\begin{array}{c}-2007,-2010,- \\
2013,-2016\end{array}$ \\
\hline & $\begin{array}{l}\text { Small } \\
\text { Office }\end{array}$ & $\begin{array}{c}511 \\
(5500)\end{array}$ & 28 & 550 & 468 & 0.100 & 0.085 \\
\hline & $\begin{array}{l}\text { Medium } \\
\text { Office }\end{array}$ & $\begin{array}{c}4982 \\
(53630)\end{array}$ & 268 & 5363 & 4559 & 0.100 & 0.085 \\
\hline & $\begin{array}{l}\text { Large } \\
\text { Office }\end{array}$ & $\begin{array}{c}46325 \\
(498640)\end{array}$ & 2493 & 49864 & 42384 & 0.100 & 0.085 \\
\hline & $\begin{array}{l}\text { Primary } \\
\text { School }\end{array}$ & $\begin{array}{c}6872 \\
(73970)\end{array}$ & 1433 & 30550 & 25041 & 0.413 & 0.339 \\
\hline & $\begin{array}{l}\text { Secondary } \\
\text { School }\end{array}$ & $\begin{array}{c}19594 \\
(210910)\end{array}$ & 6095 & 110728 & 71740 & 0.525 & 0.340 \\
\hline J & $\begin{array}{l}\text { Standalon } \\
\text { e Retail }\end{array}$ & $\begin{array}{c}2294 \\
(24690)\end{array}$ & 370 & 6795 & 5281 & 0.275 & 0.214 \\
\hline e & Strip Mall & $\begin{array}{c}2090 \\
(22500)\end{array}$ & 337 & 6750 & 5231 & 0.300 & 0.233 \\
\hline 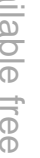 & $\begin{array}{c}\text { Quick- } \\
\text { Service } \\
\text { Restauran } \\
\text { t }\end{array}$ & $\begin{array}{c}232 \\
(2500)\end{array}$ & 94 & 1841 & 1757 & 0.736 & 0.703 \\
\hline$\underline{\underline{O}}$ & $\begin{array}{c}\text { Full- } \\
\text { Service } \\
\text { Restauran } \\
\text { t }\end{array}$ & $\begin{array}{c}511 \\
(5500)\end{array}$ & 288 & 5715 & 3872 & 1.039 & 0.704 \\
\hline $\overrightarrow{5}$ & $\begin{array}{l}\text { Small } \\
\text { Hotel }\end{array}$ & $\begin{array}{c}4014 \\
(43210)\end{array}$ & 239 & 5699 & 3941 & 0.132 & 0.091 \\
\hline 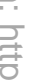 & $\begin{array}{l}\text { Large } \\
\text { Hotel }\end{array}$ & $\begin{array}{c}11345 \\
(122120)\end{array}$ & 1494 & 30247 & 19172 & 0.248 & 0.157 \\
\hline . & $\begin{array}{c}\text { Outpatien } \\
\text { t } \\
\text { Healthcar } \\
\text { e }\end{array}$ & $\begin{array}{c}511 \\
(5500)\end{array}$ & 419 & 9959 & 8389 & 0.243 & 0.207 \\
\hline b & $\begin{array}{c}\text { Warehous } \\
\text { e }\end{array}$ & $\begin{array}{c}4982 \\
(53630)\end{array}$ & 13 & 2730 & 3187 & 0.052 & 0.061 \\
\hline$\frac{\infty}{20}$ & $\begin{array}{c}\text { Mid-Rise } \\
\text { Apartmen } \\
\text { t }\end{array}$ & $\begin{array}{c}46325 \\
(498640)\end{array}$ & 67 & 1967 & 1986 & 0.058 & 0.059 \\
\hline-1 & $\begin{array}{c}\text { High-Rise } \\
\text { Apartmen } \\
\text { t }\end{array}$ & $\begin{array}{c}6872 \\
(73970)\end{array}$ & 163 & 4858 & 4927 & 0.058 & 0.058 \\
\hline जे & & $\begin{array}{l}* \text { Sta } \\
\text { cases } \\
* * 1 \mathbf{f} \\
* * * 1\end{array}$ & $\begin{array}{l}\text { ard } 62.1-2004 \\
\text { lere ventilatic } \\
\min =0.4719 \\
3 / \mathrm{min}) / \mathbf{f t}^{2} 5.0\end{array}$ & $\begin{array}{l}\text { upancy } \\
\text { ates are d } \\
32 \mathrm{~L} / \mathrm{s} \\
/ \mathrm{s}) / \mathrm{m}^{2}\end{array}$ & $\begin{array}{l}\text { ty values ensure c } \\
\text { ident on the numb }\end{array}$ & $\begin{array}{l}\text { ency in } \\
\text { buildin }\end{array}$ & $\begin{array}{l}\text { lcy numbers in } \\
\text { ants. }\end{array}$ \\
\hline
\end{tabular}

\subsection{Interior Lighting}

The average weighted lighting power density (LPD) for each prototype building model are listed in Table 3-11. Expressed in units of $\mathrm{W} / \mathrm{m}^{2}\left(\mathrm{~W} / \mathrm{ft}^{2}\right)$, LPDs for the prototype models vary with editions of Standard 90.1 and are implemented using one of the following methods: (1) the space-by-space method in Standard 90.1; (2) the building area method in Standard 90.1; or (3) a 
method combining Building America Research Benchmark (BARB) definitions and Standard 90.1 LPD specifications for corridors. All LPDs in Table 3-11 are based on zone-level LPDs weighted per fractional zone area relative to total building area, and summed across all zones resulting in an average weighted value. Power densities generally decline from Standard 90.12004 to 90.1-2016. Additional information on the average LPDs for each of the prototype models can be found by referring to Thornton, Rosenberg et al. (2011) or the downloadable PNNL prototype scorecards (US Department of Energy (DOE) n.d.).

\section{Table 3-11 Lighting Power Densities - Interior}

\begin{tabular}{|c|c|c|c|c|c|c|}
\hline \multirow[b]{2}{*}{$\begin{array}{l}\text { Prototype } \\
\text { Building }\end{array}$} & \multirow[b]{2}{*}{ Method } & \multirow[b]{2}{*}{ 90.1-2004 } & \multicolumn{4}{|c|}{ Average LPD - W/m² $\left(W / f t^{2}\right)$} \\
\hline & & & 90.1-2007 & 90.1-2010 & 90.1-2013 & 90.1-2016 \\
\hline Small Office & Building Area & $10.76(1.00)$ & $10.76(1.00)$ & $9.69(0.90)$ & $8.83(0.82)$ & $8.50(0.79)$ \\
\hline $\begin{array}{l}\text { Medium } \\
\text { Office }\end{array}$ & Building Area & $10.76(1.00)$ & $10.76(1.00)$ & $9.69(0.90)$ & $8.83(0.82)$ & $8.50(0.79)$ \\
\hline $\begin{array}{l}\text { Large } \\
\text { Office }\end{array}$ & Building Area & $10.76(1.00)$ & $10.76(1.00)$ & $9.69(0.90)$ & $8.83(0.82)$ & $8.50(0.79)$ \\
\hline $\begin{array}{c}\text { Primary } \\
\text { School }\end{array}$ & Space-by-Space & $12.81(1.19)$ & $12.81(1.19)$ & $11.30(1.05)$ & $11.41(1.06)$ & $9.04(0.84)$ \\
\hline $\begin{array}{l}\text { Secondary } \\
\text { School }\end{array}$ & pace & 1 & (1.14) & $10.33(0.96)$ & $0.23(0.95)$ & $8.27(0.77)$ \\
\hline $\begin{array}{c}\text { Standalone } \\
\text { Retail }\end{array}$ & Building Area & $16.79(1.56)$ & $16.79(1.56)$ & $16.36(1.52)$ & $14.32(1.33)$ & $12.20(1.13)$ \\
\hline Strip Mall & Building Area & $19.59(1.82)$ & 19.27 (1.79) & 18.19 (1.69) & $15.28(1.42)$ & $14.62(1.36)$ \\
\hline $\begin{array}{c}\text { Quick- } \\
\text { Service } \\
\text { Restaurant }\end{array}$ & Building Area & $17.76(1.65)$ & $17.76(1.65)$ & $10.12(0.94)$ & $10.01(0.93)$ & $9.10(0.85)$ \\
\hline $\begin{array}{l}\text { Full-Service } \\
\text { Restaurant }\end{array}$ & Building Area & $20.02(1.86)$ & $20.02(1.86)$ & $9.90(0.92)$ & $10.55(0.98)$ & $8.67(0.81)$ \\
\hline Small Hotel & Space-by-Space & $10.44(0.97)$ & $10.44(0.97)$ & $8.29(0.77)$ & $9.36(0.87)$ & $8.49(0.79)$ \\
\hline Large Hotel & Space-by-Space & $9.69(0.90)$ & $10.76(1.00)$ & $10.44(0.97)$ & $9.36(0.87)$ & $8.47(0.79)$ \\
\hline $\begin{array}{l}\text { Outpatient } \\
\text { Healthcare }\end{array}$ & Building Area & $11.84(1.10)$ & $11.84(1.10)$ & $11.30(1.05)$ & $11.52(1.07)$ & $10.45(0.97)$ \\
\hline Warehouse & Space-by-Space & $8.29(0.77)$ & $8.29(0.77)$ & $7.21(0.67)$ & $7.21(0.67)$ & $5.05(0.47)$ \\
\hline $\begin{array}{c}\text { Mid-Rise } \\
\text { Apartment }\end{array}$ & BARB/ASHRAE 90.1 & $2.91(0.27)$ & $2.91(0.27)$ & $2.91(0.27)$ & $2.91(0.27)$ & $2.91(0.27)$ \\
\hline $\begin{array}{l}\text { High-Rise } \\
\text { Apartment }\end{array}$ & BARB/ASHRAE 90.1 & $2.69(0.25)$ & $2.69(0.25)$ & $2.91(0.27)$ & $2.91(0.27)$ & $2.91(0.27)$ \\
\hline
\end{tabular}

The ASHRAE 90.1 series of building energy standards provide minimum efficiency requirements for several other aspects of a building's thermal envelope and its systems (e.g. external lighting, HVAC system fan power, etc.) which are also accounted for in the prototype building models. For information on these additional requirements not discussed in this document, please refer to Thornton, Rosenberg et al. (2011) or the downloadable PNNL prototype scorecards (US Department of Energy (DOE) n.d.). 


\section{Energy Performance Measurement}

EnergyPlus simulations for each prototype building and 90.1 edition are used to define the operating energy component in the BIRDS commercial database. The operating energy component (i.e. energy consumed during the use of the building by its occupants) was built following the framework developed in Kneifel (2010) and further expanded in Kneifel (2011a) and Kneifel (2011b), and implemented in previous versions of BIRDS (Lippiatt, Kneifel et al. 2013, Kneifel and Lavappa 2015, Kneifel, O’Rear et al. 2016). The updated BIRDS commercial database includes the results of 17100 whole-building energy simulations covering four editions of ASHRAE 90.1 (-2004, -2007, -2010, -2013, and -2016) for 15 of the 16 PNNL prototype commercial buildings, 228 cities across the United States, and 40 study period lengths.

Each building design simulated in E+ is developed by modifying 90.1-2004 compliant prototype building models based on energy efficiency requirements specified by newer editions of ASHRAE 90.1 (-2007, -2010, -2013, -2016). ASHRAE 90.1 provides both performance-based and prescriptive-based minimum requirements for the exterior building envelope R-values, roof insulation R-values, foundation insulation, window specifications, lighting efficiency, exterior lighting requirements, infiltration rates, mechanical ventilation rates, and HVAC equipment efficiencies. Each building model included in the commercial database were developed based on prescriptive requirements.

Although ASHRAE 90.1 specifies the minimum requirements for HVAC equipment efficiency, the system capacity varies across the five building energy standard editions. Changing the thermal characteristics of the building envelope alters the heating and cooling loads of the building, which changes the required capacity to meet those loads independent of the efficiency of the system. The EnergyPlus whole-building energy simulations "auto-size" the HVAC system to determine the appropriate system size to efficiently maintain the thermal comfort and ventilation requirements. The HVAC systems are automatically sized for each location by $\mathrm{E}^{+}$ based on three design day outdoor conditions that are more restrictive than those recommended by the ASHRAE Fundamentals Handbook. The cooling load is based on the Typical Meteorological Year 3 (TMY3) data: $0.4 \%$ design dry-bulb temperature and mean coincident wet-bulb temperature, and $0.4 \%$ design wet-bulb temperature and mean coincident dry bulb temperature. The heating load is based on the $99.6 \%$ dry-bulb design conditions. Both the heating and cooling auto-sizing use a sizing factor of 1.2.

Not specified by ASHRAE 90.1 are plug and process load intensities for each prototype building. External resources were used to model general loads within prototype models to account for typical building energy use and its impact on HVAC sizing and total energy consumption. Plug and process load data included in the prototype buildings is consistent with data included in the DOE commercial reference buildings (Deru, Field et al. 2011), except in the case of the mid- and high-rise apartment buildings. Plug loads for the apartment buildings are based on DOE's Building America Research Benchmark. ${ }^{6}$ Additional detailed information on plug and process

${ }^{6}$ http://www1.eere.energy.gov/buildings/building_america/index.html 
loads for the prototype building can be found in the downloadable PNNL prototype building scorecards (US Department of Energy (DOE) n.d.).

The simulations assume parameter values for the exterior envelope that represent the performance of each surface as a single material. For example, a window is represented as a single layer with parameter values that represent the combined performance characteristics of each layer of the window. The individual components of the window (e.g. panes, coatings, films, gas fill, etc.) are not specified in the simulation, only the overall U-factor, Solar Heat Gain Coefficient (SHGC), and Visual Transmittance (VT) of the window.

Five components - roof insulation, wall insulation, windows, lighting, and HVAC efficiency are changed to make the prototypical designs compliant with the latest four editions of ASHRAE 90.1. The following sections provide a summary of the building energy simulation software used in developing the new commercial database, as well as summaries of the minimum requirement ranges, excluding HVAC efficiency, for each prototype building design.

\subsection{Building Energy Simulations}

Version 8.3 of the E+ software was used to estimate the annual whole-building energy performance for each of the 17100 building designs included in the BIRDS new commercial database. The building simulation software allows users to model building heating, cooling, lighting, ventilation, and plug loads. It operates based on a series of user-defined design specifications and modeling assumptions. One of these assumptions being weather condition for a given location. The TMY3 weather files for each of the 228 locations were used for the simulations to obtain predictions of more representative years' worth of building energy performance (EnergyPlus n.d.).

\subsection{ASHRAE 90.1 Building Standard Requirements}

Again, the building designs included in BIRDS are based on the 15 commercial reference buildings described in the previous section which have been modified based on editions of ASHRAE 90.1. Minimum requirements specified by the code largely vary by edition and climate zone designation. Expressed in terms of thermal resistance ( $\mathrm{R}_{\mathrm{si}}$-value and $\mathrm{R}$-value), Table 4-1 lists the minimum requirement ranges for exterior wall insulation for all four editions of ASHRAE 90.1 considered in the database. For a given standard edition, the ranges cover requirements for Climate Zone 1 through Climate Zone 8. The ranges in insulation levels for the Mass, Metal Building, Steel Framed, and Wood Framed \& Other wall types are NR to $\mathrm{R}_{\mathrm{si}}-4.4 \mathrm{ci}$ (R-25.0ci), $\mathrm{R}_{\mathrm{si}}-2.3$ (R-13.0) to $\mathrm{R}_{\mathrm{si}}-4.4 \mathrm{ci}(\mathrm{R}-25.0 \mathrm{ci}), \mathrm{R}_{\mathrm{si}}-2.3$ (R-13.0) to $\mathrm{R}_{\mathrm{si}}-2.3+3.3 \mathrm{ci}$ (R-13.0+18.8ci), and $\mathrm{R}_{\mathrm{si}}-2.3$ (R-13.0) to $\mathrm{R}_{\mathrm{si}}-2.3+3.3 \mathrm{ci}(\mathrm{R}-13.0+18.8 \mathrm{ci})$, respectively. 
Table 4-1 Energy Efficiency Component Requirements for Alternative Building Standard Editions and Building Types - Wall Insulation

\begin{tabular}{|c|c|c|c|c|c|c|}
\hline $\begin{array}{l}\text { Design } \\
\text { Component }\end{array}$ & Parameter & $\begin{array}{l}\text { ASHRAE } \\
90.1-2004\end{array}$ & $\begin{array}{l}\text { ASHRAE } \\
\text { 90.1-2007 }\end{array}$ & $\begin{array}{l}\text { ASHRAE } \\
90.1-2010\end{array}$ & ASHRAE 90.1-2013 & $\begin{array}{l}\text { ASHRAE } \\
90.1-2016\end{array}$ \\
\hline Mass $^{\circ}$ & R-Value & $\begin{array}{l}\text { NR to } 2.7^{\circ} \\
\text { (NR to } 15.2 \text { ) }\end{array}$ & $\begin{array}{l}\text { NR to } 4.4^{\circ} \\
\text { (NR to } 25.0 \text { ) }\end{array}$ & $\begin{array}{l}\text { NR to } 4.4^{\circ} \\
\text { (NR to } 25.0)\end{array}$ & $\begin{array}{rr}\text { NR to } 3.4^{\circ} & \text { (NR to } \\
19.0) & \end{array}$ & $\begin{array}{l}\text { NR to } 3.4^{\circ} \\
\text { (NR to } 19.0 \text { ) }\end{array}$ \\
\hline $\begin{array}{l}\text { Metal } \\
\text { Building }\end{array}$ & R-Value & $\begin{array}{c}2.3 \text { to } 2.3+2.3 \\
(13.0 \text { to } \\
13.0+13.0)\end{array}$ & $\begin{array}{c}2.3 \text { to } 2.3+2.3 \\
(13.0 \text { to } \\
13.0+13.0)\end{array}$ & $\begin{array}{c}2.8 \text { to } 3.4+1.0^{\circ} \\
(16.0 \text { to } \\
19.0+5.6)\end{array}$ & $\begin{array}{c}0+1.7^{*} \text { to } 0+4.4^{\circ} \\
\left(0+9.8^{*} \text { to } 0+25.0\right)\end{array}$ & $\begin{array}{c}0+1.7^{*} \text { to } \\
0+4.4^{\circ} \\
\left(0+9.8^{*} \text { to }\right. \\
0+25.0)\end{array}$ \\
\hline $\begin{array}{l}\text { Steel } \\
\text { Framed }\end{array}$ & R-Value & $\begin{array}{c}2.3 \text { to } \\
2.3+1.8^{\circ} \\
(13.0 \text { to } \\
13.0+10.0)\end{array}$ & $\begin{array}{c}2.3 \text { to } 2.3+3.3^{\circ} \\
(13.0 \text { to } \\
13.0+18.8)\end{array}$ & $\begin{array}{c}2.3 \text { to } 2.3+3.3^{\circ} \\
(13.0 \text { to } \\
13.0+18.8)\end{array}$ & $\begin{array}{c}2.3 \text { to } 2.3+3.3^{\circ} \\
\text { (13.0 to } 13.0+18.8 \text { ) }\end{array}$ & $\begin{array}{c}2.3 \text { to } \\
2.3+3.3^{\circ} \\
(13.0 \text { to } \\
13.0+18.8)\end{array}$ \\
\hline $\begin{array}{l}\text { Wood } \\
\text { Framed } \\
\text { and Other }\end{array}$ & R-Value & $\begin{array}{c}2.3 \text { to } \\
2.3+1.3^{\circ} \\
(13.0 \text { to } \\
13.0+7.5)\end{array}$ & $\begin{array}{c}2.3 \text { to } 2.3+2.8^{\circ} \\
(13.0 \text { to } \\
13.0+15.6)\end{array}$ & $\begin{array}{c}2.3 \text { to } 2.3+2.8^{\circ} \\
(13.0 \text { to } \\
13.0+15.6)\end{array}$ & $\begin{array}{c}2.3 \text { to } 2.3+3.3^{\circ} \\
\text { (13.0 to } 13.0+18.8)\end{array}$ & $\begin{array}{c}2.3 \text { to } \\
2.3+3.3^{\circ} \\
(13.0 \text { to } \\
13.0+18.8)\end{array}$ \\
\hline \multicolumn{7}{|c|}{$\begin{array}{l}\text { Note: The units for } \mathrm{R} \text {-values are } \mathrm{m}^{2} \cdot \mathrm{K} / \mathrm{W}\left(\mathrm{ft}^{2 \cdot{ }^{\circ}} \mathbf{F} \cdot \mathbf{h} / \mathbf{B t u}\right) \\
{ }^{\circ} \text { Continuous insulation } \\
\mathrm{NR}=\text { No (insulation) requirement }\end{array}$} \\
\hline
\end{tabular}

Minimum roof insulation requirement ranges described in Table 4-2 (expressed in terms of thermal resistance ( $\mathrm{R}_{\mathrm{si}}$-value [R-value]) are categorized based on insulation method and building type. The "IEAD" approach uses continuous insulation across all structural members and ranges from $\mathrm{R}_{\mathrm{si}}-2.6$ (R-15) to $\mathrm{R}_{\mathrm{si}}-3.5$ (R-20). The "Metal Building" approach establishes minimum requirements ranging from $\mathrm{R}_{\mathrm{si}}-3.4$ (R-19) to $\mathrm{R}_{\mathrm{si}}-4.4+2.3+2.3(\mathrm{R}-25+11+11)$. Insulation installed between the roof joists (Attic \& Other) ranges from $\mathrm{R}_{\mathrm{si}}-5.3$ (R-30) to $\mathrm{R}_{\mathrm{si}}-10.6$ (R-60). The Metal Building option is the only one of the three that uses either a liner system roof assembly ${ }^{7}$ or both a liner system and filled cavity roof assemblies.

Table 4-2 Energy Efficiency Component Requirements for Alternative Building Standard Editions and Building Types - Roof Insulation

\begin{tabular}{|c|c|c|c|c|c|c|}
\hline $\begin{array}{l}\text { Design } \\
\text { Component }\end{array}$ & $\begin{array}{l}\text { Building } \\
\text { Type }\end{array}$ & $\begin{array}{l}\text { ASHRAE } \\
\text { 90.1-2004 }\end{array}$ & $\begin{array}{l}\text { ASHRAE } \\
\text { 90.1-2007 }\end{array}$ & $\begin{array}{l}\text { ASHRAE } \\
\text { 90.1-2010 }\end{array}$ & $\begin{array}{l}\text { ASHRAE } \\
\text { 90.1-2013 }\end{array}$ & $\begin{array}{l}\text { ASHRAE } \\
90.1-2016\end{array}$ \\
\hline IEAD $^{\circ}$ & R-Value & $\begin{array}{c}2.6 \text { to } 3.5 \\
(15.0 \text { to } 20.0)\end{array}$ & $\begin{array}{c}2.6 \text { to } 3.5 \\
\text { (15.0 to } 20.0)\end{array}$ & $\begin{array}{c}2.6 \text { to } 3.5 \\
\text { (15.0 to } 20.0)\end{array}$ & $\begin{array}{l}3.5^{\circ} \text { to } 6.2^{\circ} \\
(20.0 \text { to } 35.0)\end{array}$ & $\begin{array}{l}3.5^{\circ} \text { to } 6.2^{\circ} \\
(20.0 \text { to } 35.0)\end{array}$ \\
\hline $\begin{array}{l}\text { Metal } \\
\text { Building }\end{array}$ & R-Value & $\begin{array}{c}3.4 \text { to } 2.3+3.4 \\
(19.0 \text { to } \\
13.0+19.0)\end{array}$ & $\begin{array}{c}3.4 \text { to } 2.3+3.4 \\
(19.0 \text { to } \\
13.0+19.0)\end{array}$ & $\begin{array}{c}3.4 \text { to } 2.3+3.4^{*} \\
(19.0 \text { to } \\
11.0+19.0)\end{array}$ & $\begin{array}{c}1.8+3.4^{* *} \text { to } \\
4.4+2.3+2.3^{*} \\
(10.0+19.0 \text { to } \\
25.0+11.0+11.0)\end{array}$ & $\begin{array}{c}1.8+3.4^{* *} \text { to } \\
4.4+2.3+2.3^{*} \\
(10.0+19.0 \text { to } \\
25.0+11.0+11.0)\end{array}$ \\
\hline $\begin{array}{l}\text { Attic \& } \\
\text { Other }\end{array}$ & R-Value & $\begin{array}{c}5.3 \text { to } 6.7 \\
\text { (30.0 to } 38.0)\end{array}$ & $\begin{array}{c}5.3 \text { to } 8.6 \\
\text { (30.0 to } 49.0)\end{array}$ & $\begin{array}{c}5.3 \text { to } 8.6 \\
\text { (30.0 to } 49.0 \text { ) }\end{array}$ & $\begin{array}{c}6.7 \text { to } 10.6 \\
\text { (38.0 to } 60.0)\end{array}$ & $\begin{array}{c}6.7 \text { to } 10.6 \\
\text { (38.0 to } 60.0 \text { ) }\end{array}$ \\
\hline
\end{tabular}

\footnotetext{
${ }^{7}$ A liner system is a type of roof insulation assembly that uses a continuous flexible vapor retarder membrane installed below by the purlins that is fastened in place by a support structure such steel bands.
} 
Minimum window requirement ranges for the four most recent editions of ASHRAE 90.1 are listed in Table 4-3. Windows are characterized in terms of U-Factor and SHGC. For both nonresidential and residential windows, U-Factors range from $1.42 \mathrm{~W} / \mathrm{m}^{2} \cdot \mathrm{K}\left(0.25 \mathrm{Btu} / \mathrm{h} \cdot \mathrm{ft}^{2} \cdot{ }^{\circ} \mathrm{F}\right)$ to $7.21 \mathrm{~W} / \mathrm{m}^{2} \cdot \mathrm{K}\left(1.27 \mathrm{Btu} / \mathrm{h} \cdot \mathrm{ft}^{2} \cdot{ }^{\circ} \mathrm{F}\right)$. Values from the table indicate that the required window heat insulation performance either improves or remains constant with the introduction of a new series of 90.1 standard requirements. The range of solar heat gain coefficients (SHGC) remain the same across standard editions, ranging from 0.25 to NR, except for 90.-2016 that lowers the most restrictive requirement to 0.22 .

\section{Table 4-3 Energy Efficiency Component Requirements for Alternative Building Standard Editions and Building Types - Windows}

\begin{tabular}{|c|c|c|c|c|c|c|c|}
\hline Building Type & Parameter & Units & $\begin{array}{l}\text { ASHRAE } \\
90.1-2004\end{array}$ & $\begin{array}{l}\text { ASHRAE } \\
\text { 90.1-2007 }\end{array}$ & $\begin{array}{l}\text { ASHRAE } \\
90.1-2010\end{array}$ & $\begin{array}{l}\text { ASHRAE } \\
90.1-2013\end{array}$ & $\begin{array}{l}\text { ASHRAE } \\
90.1-2016\end{array}$ \\
\hline \multirow[t]{2}{*}{$\begin{array}{l}\text { Nonresidential } \\
\text { / Residential }\end{array}$} & U-Factor & $\begin{array}{l}\mathrm{W} /\left(\mathrm{m}^{2} \cdot \mathrm{K}\right) \\
\left(\mathrm{Btu} /\left(\mathrm{h} \cdot \mathrm{ft}^{2}{ }^{\circ} \mathrm{F}\right)\right)\end{array}$ & $\begin{array}{c}1.99 \text { to } 7.21 \\
(0.35 \text { to } \\
1.27)\end{array}$ & $\begin{array}{c}1.99 \text { to } 6.81 \\
(0.35 \text { to } \\
1.20)\end{array}$ & $\begin{array}{c}1.99 \text { to } 6.81 \\
(0.35 \text { to } \\
1.20)\end{array}$ & $\begin{array}{c}1.82 \text { to } 6.25 \\
(0.32 \text { to } \\
1.10)\end{array}$ & $\begin{array}{c}1.42 \text { to } 6.25 \\
(0.25 \text { to } \\
1.10)\end{array}$ \\
\hline & SHGC & Fraction & $\begin{array}{c}0.25 \text { to } \\
\mathrm{NR} \dagger\end{array}$ & $\begin{array}{c}0.25 \text { to } \\
\mathrm{NR} \dagger\end{array}$ & $\begin{array}{c}0.25 \text { to } \\
\mathrm{NR} \dagger\end{array}$ & $\begin{array}{c}0.25 \text { to } \\
\mathrm{NR} \dagger\end{array}$ & 0.22 to 0.45 \\
\hline
\end{tabular}

Unlike the previous three energy efficiency components, requirements for interior lighting efficiency (expressed in terms of LPD) varies only across ASHRAE 90.1 editions and not by climate zone. Interior lighting requirements are defined by ASHRAE standards using one of two methodologies: (1) space-by-space method or (2) building area method. The space-by-space method associates a LPD $\left(\mathrm{W} / \mathrm{ft}^{2}\right)$ with a defined list of possible space types in a building. The building area method associates a LPD $\left(\mathrm{W} / \mathrm{ft}^{2}\right)$ with a list of possible whole-building types. For detailed information on interior lighting efficiency requirements for each of the 90.1 editions considered in BIRDS, refer to ASHRAE Standard 90.1-2004, 90.1-2007, 90.1-2010, 90.1-2013, and 90.1-2016 (ASHRAE/IESNA Standard Project Committee 90.1 1999, ASHRAE/IESNA Standard Project Committee 90.1 2004, ASHRAE/IESNA Standard Project Committee 90.1 2007, ASHRAE/IESNA Standard Project Committee 90.1 2010, ASHRAE/IESNA Standard Project Committee 90.1 2013, ASHRAE/IESNA Standard Project Committee 90.1 2016). 


\section{Environmental Performance Measurement}

BIRDS evaluates the environmental performance of whole-buildings using a life-cycle assessment (LCA) approach, which is based on International Organization for Standardization (ISO) standards. The general LCA methodology involves four steps (International Organization for Standardization (ISO) 2006a, International Organization for Standardization (ISO) 2006b).

1. Goal and scope definition

2. Inventory analysis

3. Impact assessment

4. Interpretation

The goal and scope definition step spells out the purpose of the study and its breadth and depth. The inventory analysis step identifies and quantifies the environmental inputs and outputs associated with a building over its entire life-cycle. Environmental inputs include water, energy, land, and other resources. Outputs include releases to air, land, and water. However, it is not these inputs and outputs, or inventory flows, which are of primary interest. The interest lies in their consequences, or impacts on the environment. Thus, the impact assessment step characterizes these inventory flows in relation to a set of environmental impacts. For example, the impact assessment step might relate carbon dioxide emissions, a flow, to global warming, an impact. Finally, the interpretation step examines the environmental impacts in accordance with the goals of the LCA study.

\subsection{Goal and Scope Definition}

The goal of BIRDS LCAs is to generate environmental performance scores for a range of U.S. building types, each designed to meet four alternative levels of operating energy efficiency. These results will be reported alongside economic performance scores to help designers, investors, and policymakers develop business cases for high-performance green buildings.

The scoping phase of any LCA involves defining the boundaries of the product system, or, in the case of BIRDS, the building under study. In traditional bottom-up LCAs, the setting of the boundary conditions requires expert judgment by the analyst because consideration must be given to the various unit processes related to the construction of the building (e.g. asphalt production for input to the manufacturing of facing for fiberglass batt insulation). Each unit process involves many inventory flows, some of which themselves involve other, subsidiary unit processes. Because including an ever-expanding number of unit processes in LCAs is not feasible, the product system's supply chain links are truncated at some point to include only those judged to make non-negligible contributions to the product system. The analyst typically uses mass, energy, and/or cost contributions as decision criteria. Use of different boundary setting criteria is one of the main reasons LCA results from different studies are often incomparable.

One important advantage of the BIRDS hybrid approach (discussed further in Section 5.2) is that it addresses the bottom-up issue of truncation in supply chain links, thereby improving 
consistency in system boundary selections. Through the hybridization process, truncated supply chain links are connected to the background U.S. economic system represented by the top-down data. These linkages follow the metabolic structure of the U.S. economy, thereby benefitting from a more complete system definition including potentially thousands of supply chain interactions.

Due to their long service lives, buildings are unique when it comes to the end-of-life stage of the life-cycle. For most non-consumable product LCAs, end-of-life waste flows are included in the inventory analysis for full coverage of the life-cycle. If there is an active recycling market that diverts some of the product from the waste stream, that portion of the product's end-of-life flows can be ignored. In BIRDS, however, a commercial building lifetime is greater than the maximum study period length considered in BIRDS (40 years). Therefore, $100 \%$ of each building is considered "recycled" at the end of the study period and there are no end-of-life waste flows allocated to the building at the end of the BIRDS study period. Rather, end-of-life waste flows should be allocated to a different "product," representing use of the building from the end of the study period to the end of the building service life. Similarly, the environmental burdens from building construction are allocated only to its first use (equally distributed across each year of the selected study period); LCAs for all subsequent uses should be treated as free of these initial construction burdens. This effectively credits the use of existing buildings over new construction and ensures there will be no double counting if existing building LCAs are included in future versions of BIRDS.

Defining the unit of comparison is an important task in the goal and scoping phase of LCA. The basis for all units of comparison is the functional unit, defined so that the systems compared are true substitutes for one another. In the BIRDS model, the functional unit is construction and use of one building prototype over a user-defined study period. The functional unit provides the critical reference point to which the LCA results are scaled.

Scoping also involves setting data requirements. With respect to geographic coverage, the BIRDS inventory data are generally U.S. average data. An exception is made for the electricity production inventory data applied to a building's use of electricity. These data are customized to each U.S. state using U.S. EPA eGRID data (U.S. EPA 2008). In terms of technology coverage, the top-down inventory data represent the mix of technologies in place as of $2002^{8}$. For the bottom-up inventory data on building energy technologies, the most representative technology for which data are available is evaluated.

\subsection{Life-cycle Inventory Analysis}

BIRDS applies a hybridized life-cycle assessment approach. The approach is hybridized in the sense that a mixture of top-down and bottom-up data are collected and systematically integrated in the inventory analysis LCA step. Traditional process-based LCAs gather data by modeling all the in-scope industrial processes involved in a product's production (raw materials acquisition,

\footnotetext{
${ }^{8}$ More recent data is not available due to funding limitations of developing environmental inventory data for the most recent U.S. Economic Census.
} 
materials processing, manufacture, transportation), use, and waste management. For each industrial process, the analyst collects very detailed, bottom-up data on all its inputs from the environment (e.g., materials, fuel, water, land) and outputs to the environment (e.g., products, water effluents, air emissions, waste). This process is summarized in Figure 5-1.

To address the complexities of a whole building, BIRDS takes a new, multi-layered approach to inventory analysis. Since a building's operating energy efficiency has an important influence on its sustainability performance, and energy efficiency is largely driven by the building's energy technologies, BIRDS pays special attention to the materials used in those building components. Specifically, BIRDS uses detailed life-cycle inventory (LCI) data for a range of energy technology packages that have been analyzed at the traditional, bottom-up LCA level. These energy technology packages are used to meet the 4 levels of energy efficiency based on editions of ASHRAE 90.1 simulated for each building type in 228 different U.S. locations. The bottom-up approach is also used to gather inventory data for a building's use of electricity and natural gas over the study period. These bottom-up BIRDS data were developed under contract to NIST by Four Elements Consulting, LLC, of Seattle, Washington, and are documented in Section 5.5. For all other building constituents, industry average LCI data are gathered from the top-down approach and then systematically combined with bottom-up data into a comprehensive, hybrid LCI for a whole building.

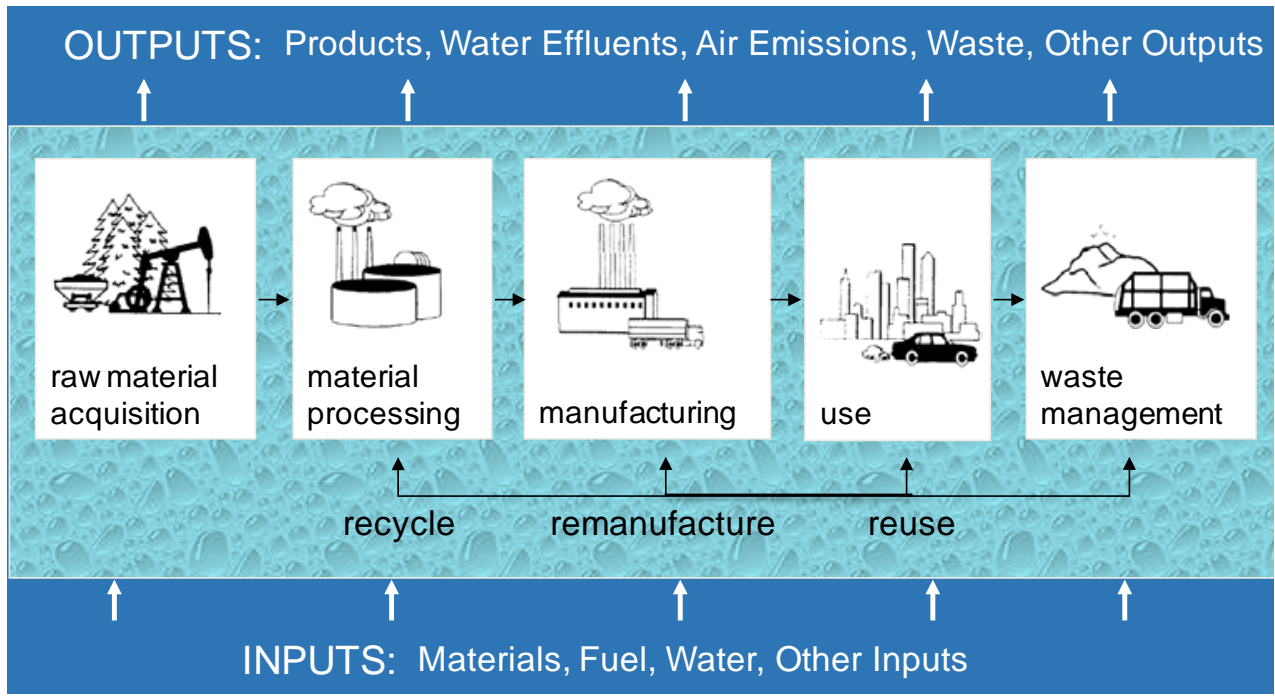

Figure 5-1 Compiling LCA Inventories of Environmental Inputs and Outputs

The inventory data items collected through the bottom-up and top-down approaches are identical - for example, kilogram $(\mathrm{kg})$ carbon dioxide, kWh primary energy consumption - but some of the data sources are quite different. The systematic hybridization of the data sets bridges these differences to yield coherent and consistent BIRDS life-cycle inventories for a wide variety of new commercial and residential buildings. The LCAs for the buildings are then completed by applying conventional methods of life-cycle impact assessment (LCA) and interpretation to the hybrid inventory data. 
An economy's accounting structure provides a cost-effective top-down approach to LCA inventory data collection. Many developed economies maintain economic input-output (I-O) accounts that trace the flow of goods and services throughout industries. Much the same way that a product's production can be traced upstream through its supply chain, an industry's production can be traced upstream through its supply chain. The U.S. Census Bureau conducts an Economic Census of U.S. industry every five years that establishes industry linkages. Covering $97 \%$ of business receipts, the census reaches nearly all U.S. business establishments. Based on the detailed data collected, the U.S. Bureau of Economic Analysis (BEA) creates what are called Input-Output Accounts, or "I-O tables," for the U.S. economy.

The U.S. I-O tables show how around 500 industries provide input to, and use output from, each other to produce Gross Domestic Product (GDP) - the total value of the consumption of goods and services in a year. These tables, for example, can show how $\$ 100$ million of U.S. economic output in the commercial building construction sector traces back through its direct monetary inputs - from the construction process itself- to its indirect inputs from contributing sectors such as the steel, concrete, lumber, and plastics industries. Economic output from the steel, concrete, lumber, and plastics industries, in turn, can be traced back through those supply chains such as mining, forestry, fuel extraction, and so on.

While BEA provides these I-O tables in purely monetary terms, academics have successfully developed “environmentally-extended” I-O tables (Suh 2005, Hendrickson, Lave et al. 2006, Suh 2010). These top-down tables tap into a wide range of national environmental statistics to associate environmental inputs and outputs with economic activity in industry sectors, including use of raw materials, fuel, water, and land and releases of water effluents, air emissions, and waste. BIRDS uses environmentally-extended I-O tables for the U.S. construction industry developed under contract to NIST by Industrial Ecology Research Services of Goleta, California. These tables are based on the 2007 release of the 2002 BEA I-O data, the latest available at the time of development, and quantify 6204 environmental inputs and outputs occurring throughout production supply chains.

To understand the contribution of building construction to the nation's environmental footprint (impact), it is useful to focus on the concept of "final demand." The BEA's monetary I-O tables use GDP to measure final demand. This value consists of spending and investment by consumers, businesses, and government, as well as net exports. Since final demand is satisfied through annual production - goods and services need to be produced before they can be bought each industry's value-added, or "direct” contribution to GDP, reflects its share of final demand, and will be referred to as GDP for the remainder of this document.

The environmentally-extended I-O tables translate economic activity into environmental terms, or monetary GDP into environmental GDP (eGDP). In LCA terms, the construction industry's contribution to eGDP is not limited to the direct impact from value-added construction processes and activities. Its contribution to eGDP also includes the indirect impacts stemming from contributions by upstream construction supply chains up to and including raw materials acquisition. The supply chain relationships built into the environmentally-extended I-O tables enable estimation of construction industry impacts on this cumulative, life-cycle basis. Figure 
5-2 illustrates these supply chain relationships for some of the inputs to ready-mix concrete manufacturing, an indirect construction industry input.

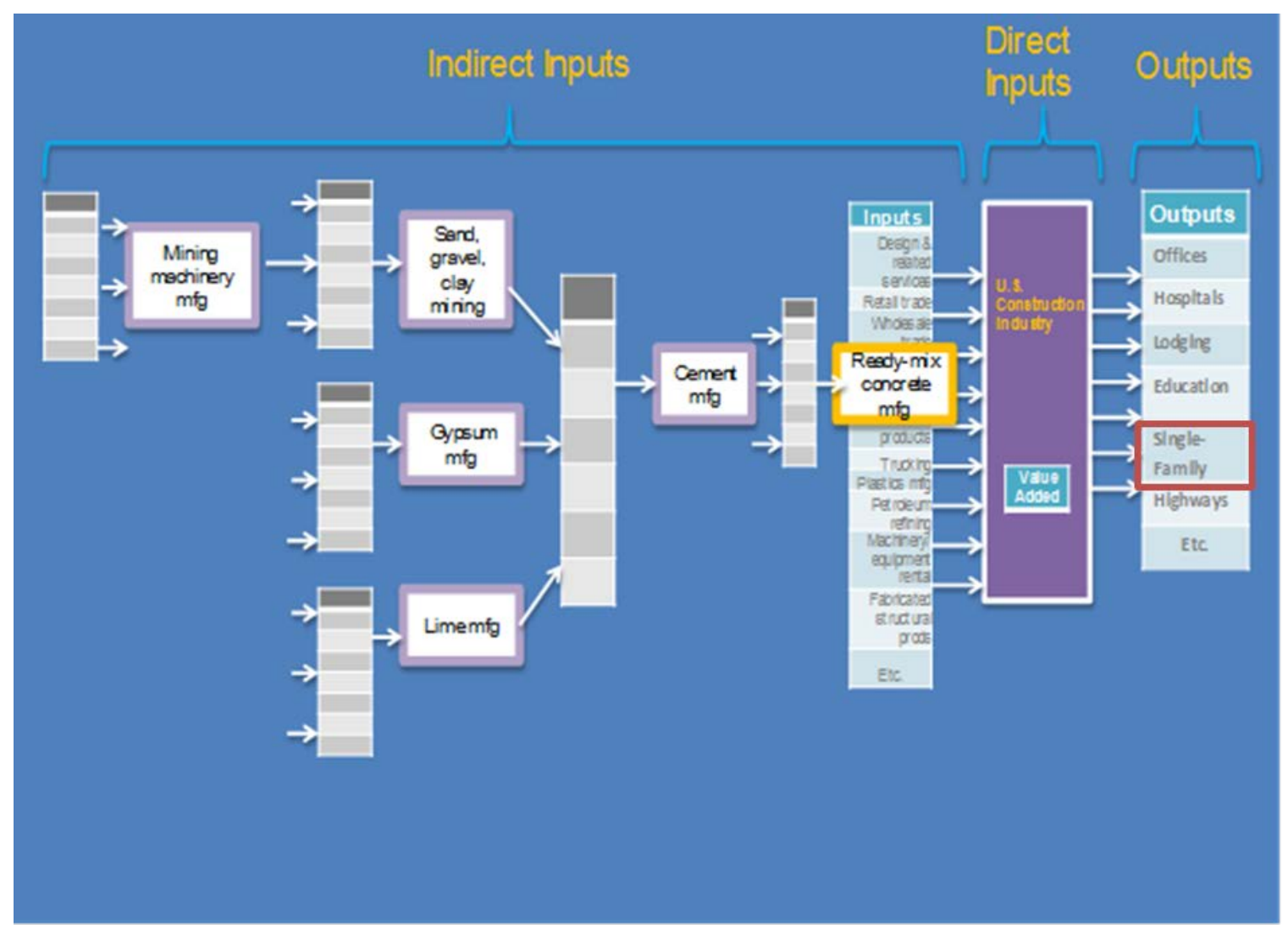

Figure 5-2 Illustration of Supply Chain Contributions to U.S. Construction Industry

The environmentally extended I-O tables classify U.S. construction into 42 distinct industry outputs. In this new version of BIRDS, top-down inventory data represent the extended I-O table level of detail for the construction, maintenance, and repair associated with the 8 industry outputs shown in Table 5-1. The first 6 outputs correspond to the occupancy types covered by the 15 prototype buildings in the updated commercial database. The last two correspond to maintenance and repair (M\&R) activities in those buildings. For routine $M \& R$, nonresidential M\&R output applies to all but the lodging occupancy prototypes. For these, residential M\&R output applies. For all the construction industry outputs, the baseline top-down inventory data are expressed in terms of life-cycle environmental flows per dollar of construction. 
Table 5-1 Construction Industry Outputs Mapped to BIRDS Building Types

\begin{tabular}{|c|c|c|c|}
\hline Construction Type & Industry Output & Occupancy & BIRDS Building Type \\
\hline \multirow{14}{*}{ New Construction } & \multirow{3}{*}{$\begin{array}{l}\text { New office buildings, including } \\
\text { financial buildings }\end{array}$} & \multirow{3}{*}{ Office } & OFFICE01 \\
\hline & & & OFFICE03 \\
\hline & & & OFFICE12 \\
\hline & \multirow{2}{*}{$\begin{array}{l}\text { New multi-merchandise } \\
\text { shopping }\end{array}$} & \multirow[b]{2}{*}{ Mercantile } & RETAIL01 \\
\hline & & & STRPMALL01 \\
\hline & \multirow{2}{*}{$\begin{array}{l}\text { New food and beverage } \\
\text { establishments }\end{array}$} & \multirow[b]{2}{*}{ Food Service } & FFRSTR01 \\
\hline & & & SDRSTR01 \\
\hline & \multirow{2}{*}{$\begin{array}{l}\text { New educational and vocational } \\
\text { structures }\end{array}$} & \multirow[b]{2}{*}{ Education } & ELEMSC01 \\
\hline & & & HIGHSC02 \\
\hline & \multirow{2}{*}{ New lodging } & \multirow{4}{*}{ Lodging } & HOTEL04 \\
\hline & & & HOTEL06 \\
\hline & \multirow{2}{*}{$\begin{array}{l}\text { New multifamily residential } \\
\text { structures }\end{array}$} & & APART04 \\
\hline & & & APART10 \\
\hline & New warehouses & Warehouse & WAREH01 \\
\hline \multirow{5}{*}{$\begin{array}{l}\text { M\&R } \\
\text { Construction }\end{array}$} & \multirow{4}{*}{$\begin{array}{l}\text { Residential maintenance and } \\
\text { repair construction }\end{array}$} & \multirow{4}{*}{ Lodging } & HOTEL04 \\
\hline & & & HOTEL06 \\
\hline & & & APART04 \\
\hline & & & APART10 \\
\hline & Nonresidential maintenance & All Others & All Others \\
\hline
\end{tabular}

One advantage of the BIRDS approach is the economic dimension built into the top-down inventory data. These data are directly associated with U.S. economic data, permitting seamless integration of the economic dimension in the BIRDS sustainability measurement system. The top-down inventory values on a per-dollar basis are multiplied by the corresponding BIRDS construction, maintenance, and repair costs to translate them into the LCA functional unit representing the whole-building over a user-defined study period.

For more information on the mathematics, accounting structure, and step-by-step process under which the BIRDS hybrid environmental database is built, see Suh and Lippiatt (2012).

\subsection{Life-cycle Impact Assessment}

Environmental impacts from building construction and use derive from the 6204 inputs and outputs occurring throughout production supply chains, as quantified in the hybrid BIRDS LCI. The impact assessment step of LCA quantifies the potential contribution of these inventory items to a range of environmental impacts. The approach preferred by most LCA practitioners and scientists today involves a two-step process:

- Classification of inventory flows that contribute to specific environmental impacts. For example, greenhouse gases such as carbon dioxide, methane, and nitrous oxide are classified as contributing to global warming. 
- Characterization of the potential contribution of each classified inventory flow to the corresponding environmental impact. This results in a set of indices, one for each impact, which is obtained by weighting each classified inventory flow by its relative contribution to the impact. For instance, the Global Warming Potential index is derived by expressing each greenhouse gas in terms of its equivalent amount of carbon dioxide heat trapping potential.

There are two general applications of this life-cycle impact assessment (LCIA) approach: midpoint-level and endpoint-level analyses. An endpoint-level analysis attempts to measure the ultimate damage that each environmental input and output in the inventory will have along the cause-effect chain. Methods of this type include just a few impact categories, such as damage to human health, ecosystems, and resource availability that are easier to interpret in the final step of life-cycle assessment. This approach is criticized for the numerous assumptions, value judgments, and gaps in coverage of the underlying damage models. A midpoint-level analysis, on the other hand, selects points along the cause-effect chain at which more certain and comprehensive assessments may be carried out. While this approach generates many impact categories and makes life-cycle interpretation more difficult, it is more scientifically defensible. Even so, a midpoint-level analysis does not offer the same degree of relevance for all environmental impacts. For global and regional effects (e.g., global warming and acidification) the method provides an accurate description of the potential impact. For impacts dependent upon local conditions (e.g., smog), it may result in an oversimplification of the actual impacts because the indices are not tailored to localities. Note that some impact assessments apply a mix of midpoint and endpoint approaches.

\subsubsection{BIRDS Impact Assessment}

BIRDS uses a midpoint-level analysis to translate its 6204 environmental inputs and outputs into a manageable set of science-based measurements across 12 environmental impacts. BIRDS primarily uses the U.S. Environmental Protection Agency's (EPA) Tool for the Reduction and Assessment of Chemical and other environmental Impacts (TRACI) version 2.0 set of state-ofthe-art, peer-reviewed U.S. life-cycle impact assessment methods (Bare 2011). Since TRACI 2.0 does not include land and water use, these two important resource depletion impacts are assessed using other characterization methods (Guinée 2002, Goedkoop, Heijungs et al. 2008). Together these methods are used to develop BIRDS performance metrics indicating the degree to which construction and use of a building contributes to each environmental impact. What follows are brief descriptions of the 12 BIRDS impact categories.

\subsubsection{Impact Categories}

\subsection{Global Warming}

The Earth absorbs radiation from the Sun, mainly at the surface. This energy is then redistributed by the atmosphere and ocean and re-radiated to space at longer wavelengths. Greenhouse gases in the atmosphere, principally water vapor, but also carbon dioxide, methane, chlorofluorocarbons, and ozone, absorb some of the thermal radiation. The absorbed energy is 
re-radiated in all directions, downwards as well as upwards, such that the radiation that is eventually lost to space is from higher, colder levels in the atmosphere. The result is that the surface loses less heat to space than it would in the absence of the greenhouse gases and consequently stays warmer than it would be otherwise. This phenomenon, which acts like a 'blanket' around the Earth, is known as the greenhouse effect.

The greenhouse effect is a natural phenomenon. The environmental issue is the change in the greenhouse effect due to emissions (an increase in the effect) and absorptions (a decrease in the effect) attributable to humans. A general increase in temperature can alter atmospheric and oceanic temperatures, which can potentially lead to alteration of natural circulations and weather patterns. A rise in sea level is also predicted from an increase in temperature due to thermal expansion of the oceans and the melting of polar ice sheets.

\subsection{Primary Energy Consumption}

Primary energy consumption leads to fossil fuel depletion when fossil fuel resources are consumed at rates faster than nature renews them. Some experts believe fossil fuel depletion is fully accounted for in market prices. That is, market price mechanisms are believed to take care of the scarcity issue, with price being a measure of the level of depletion of a resource and the value society places on that depletion. However, price is influenced by many factors other than resource supply, such as resource demand and non-perfect markets (e.g., monopolies and subsidies). The primary energy consumption metric is used to account for the resource depletion aspect of fossil fuel extraction.

\subsection{Human Health - Criteria Air Pollutants}

These pollutants can arise from many activities including combustion, vehicle operation, power generation, materials handling, and crushing and grinding operations. They include coarse particles known to aggravate respiratory conditions such as asthma, and fine particles that can lead to more serious respiratory symptoms and disease.

\subsection{Human Health - Cancer Effects}

These effects can arise from exposure to industrial and natural substances, and can lead to illness, disability, and death. Its assessment is based on the global consensus model known as USEtox, which describes the fate, exposure and effects of thousands of chemicals (Rosenbaum, Huijbregts et al. 2011).

\subsection{Water Consumption}

Water resource depletion has not been routinely assessed in LCAs to date, but researchers are beginning to address this issue to account for areas where water is scarce, such as the Western United States. While some studies use water withdrawals to evaluate this impact, a more refined analysis considers that a portion of water withdrawn may be returned through evapotranspiration (the sum of evaporation from surface water, soil, and plant leaves). BIRDS uses the latter approach to measure water consumption, or water withdrawn net of evapotranspiration. BIRDS evaluates water consumption from cradle to grave, including water consumption during building use. 


\subsection{Ecological Toxicity}

Measures of ecological toxicity consider the potential of pollutants from industrial sources to harm land- and water-based ecosystems. Its assessment is based on the global consensus model known as USEtox, which describes the fate, exposure and effects of thousands of chemicals.

\subsection{Eutrophication Potential}

Eutrophication is the addition of mineral nutrients to the soil or water. In both media, the addition of large quantities of mineral nutrients, such as nitrogen and phosphorous, results in generally undesirable shifts in the number of species in ecosystems and a reduction in ecological diversity. In water, it tends to increase algae growth, which can lead to a lack of oxygen and subsequent death of species like fish.

\subsection{Land Use}

This impact category measures the use of land resources in hectares by humans which can lead to undesirable changes in habitats. Note that the BIRDS land use approach does not consider the original condition of the land, the extent to which human activity changes the land, or the length of time required to restore the land to its original condition. As impact assessment science continues to evolve, it is hoped that these potentially important factors will become part of BIRDS land use assessment.

\subsection{Human Health - Non-cancer Effects}

The effects can arise from exposure to industrial and natural substances, and range from transient irritation to permanent disability and even death. Its assessment is based on the global consensus model known as USEtox, which describes the fate, exposure and effects of thousands of chemicals.

\subsection{Smog Formation}

Smog forms under certain climatic conditions when air emissions (e.g. $\mathrm{NO}_{\mathrm{x}}$, VOCs) from industry and transportation are trapped at ground level where they react with sunlight. Smog leads to harmful impacts on human health and vegetation.

\subsection{Acidification Potential}

Acidifying compounds may, in a gaseous state, either dissolve in water or fix on solid particles. These compounds reach ecosystems through dissolution in rain or wet deposition and can affect trees, soil, buildings, animals, and humans. The two compounds principally involved in acidification are sulfur and nitrogen compounds, with their principal human source being fossil fuel and biomass combustion. Other compounds released by human sources, such as hydrogen chloride and ammonia, also contribute to acidification.

\subsection{Ozone Depletion}

Ozone depletion is the thinning of the stratospheric ozone layer, allows more harmful short wave radiation to reach the Earth's surface, potentially causing undesirable changes in ecosystems, agricultural productivity, skin cancer rates, and eye cataracts, among other issues. 


\subsubsection{Computational Algorithms}

There are six building components represented in the BIRDS LCIA calculations for wholebuildings:

- Baseline building: new construction (Base_New)

- Baseline building: maintenance and repair over study period (Base_M\&R)

- Energy technology package: new construction (ETP_New)

- Energy technology package: annual maintenance and repair (ETP_M\&R)

- Annual operating energy use: electricity (ELEC)

- Annual operating energy use: natural gas (NG)

The hybridized LCI data for each component are expressed in different units. For example, the baseline building inventories are given on a per-dollar basis, the energy technology package inventories on a per-physical unit basis (e.g. area or system), and the operating energy use inventories on a per-unit of energy basis. Thus, each requires its own LCIA computational algorithm as shown in Table 5-2. These calculations ensure that after adjusting for study period length, all LCIA results are expressed in the consistent functional unit defined for BIRDS: construction and use of one building prototype over a user-defined study period.

\section{Table 5-2 BIRDS LCIA by Building Component}

\begin{tabular}{|c|c|c|c|c|}
\hline $\begin{array}{l}\text { Building } \\
\text { Component }\end{array}$ & \multicolumn{3}{|c|}{ LCIA Equation } & \multirow{4}{*}{$\begin{array}{l}\text { Notation } \\
\text { LCIA=classified and characterized life- } \\
\text { cycle inventories } \\
\text { c=construction type code, 1=new, 2=M\&R } \\
\text { E=electricity } \\
\text { ETP=energy technology product } \\
\text { FU=functional unit }\end{array}$} \\
\hline Base_New & $\operatorname{LCIA}_{i, j, c=1}$ & $=$ & $\left(\operatorname{LCIA}_{\mathrm{i}, \mathrm{j}, \mathrm{c}=1} / \$\right) \cdot \$ \mathrm{i}_{\mathrm{i}, \mathrm{c}=1}$ & \\
\hline Base_M\&R & $\operatorname{LCIA}_{i, j, c=2, y r}$ & $=$ & $\left(\operatorname{LCIA}_{\mathrm{i}, \mathrm{j}, \mathrm{c}=2} / \$\right) \cdot \$_{\mathrm{i}, \mathrm{c}=2, \mathrm{yr}}$ & \\
\hline ETP_New & $\operatorname{LCIA}_{\mathrm{i}, \mathrm{j}, \mathrm{c}=1, \mathrm{x}, \mathrm{K}, \mathrm{T}}$ & $=$ & $\begin{array}{l}\sum\left(\mathrm{LCIA}_{\mathrm{j}, \mathrm{c}=1, \mathrm{ET}(\mathrm{i}, \mathrm{x}, \mathrm{K}, \mathrm{T})} / \mathrm{FU}\right) \cdot \mathrm{FU}_{\mathrm{i}, \mathrm{T}} \\
\text { from } \mathrm{T}=1 \text { to } 6\end{array}$ & \\
\hline ETP_M\&R & $\mathrm{LCIA}_{\mathrm{i}, \mathrm{j}, \mathrm{c}=2, \mathrm{x}, \mathrm{K}, \mathrm{T}} / \mathrm{yr}$ & $=$ & $\begin{array}{l}\sum\left(\mathrm{LCIA}_{\mathrm{j}, \mathrm{c}=2, \mathrm{ET}(\mathrm{i}, \mathrm{x}, \mathrm{K}, \mathrm{T})} / \mathrm{FU} / \mathrm{yr}\right) \cdot \mathrm{FU}_{\mathrm{i}, \mathrm{T}} \\
\text { from } \mathrm{T}=1 \text { to } 6\end{array}$ & $\begin{array}{l}\mathrm{j}=\text { environmental impact, } \mathrm{j}=1 \text { to } 12 \\
\mathrm{~K}=\text { building design }\end{array}$ \\
\hline ELEC & $\operatorname{LCIA}_{\mathrm{i}, \mathrm{j}, \mathrm{x}, \mathrm{K}} / \mathrm{yr}$ & $=$ & $\left(\mathrm{LCIA}_{\mathrm{j}, \mathrm{s}} / \mathrm{BTU}_{\mathrm{E}}\right) \cdot\left(\mathrm{BTU}_{\mathrm{E}, \mathrm{i}, \mathrm{x}, \mathrm{K}} / \mathrm{yr}\right)$ & $\begin{array}{l}\mathrm{NG}=\text { natural gas } \\
\mathrm{s}=\mathrm{U} . S . \text { state, }\end{array}$ \\
\hline NG & $\mathrm{LCIA}_{\mathrm{i}, \mathrm{j}, \mathrm{x}, \mathrm{K}} / \mathrm{yr}$ & $=$ & $\left(\mathrm{LCIA}_{\mathrm{j}} / \mathrm{BTU}_{\mathrm{NG}}\right) \cdot\left(\mathrm{BTU}_{\mathrm{NG}, \mathrm{i}, \mathrm{x}, \mathrm{K}} / \mathrm{yr}\right)$ & $\begin{array}{l}\mathrm{T}=\text { energy technology group, } \mathrm{T}=1 \text { to } 10 \\
\mathrm{x}=\text { building location }\end{array}$ \\
\hline
\end{tabular}

*Energy technology groups and their functional units are wall insulation (area), foundation insulation (area), attic insulation (area), air leakage rate (area), windows (area), lighting (watt) heating, cooling, and ventilation equipment (no. of units), solar thermal (no. of units), and solar photovoltaic (no. of units).

\subsubsection{BIRDS Normalization}

Once impacts have been classified and characterized, the resulting LCIA metrics are expressed in incommensurate units. For example, global warming is expressed in carbon dioxide equivalents $\left(\mathrm{CO}_{2} \mathrm{e}\right)$ and acidification in hydrogen ion equivalents. To assist in the next LCA step, interpretation, these metrics are often placed on the same scale through normalization. 
The EPA has developed "normalization references" corresponding to its TRACI set of impact assessment methods (Bare, Gloria et al. 2006). These U.S. data are updated and expanded for use in BIRDS. Shown in Table 5-3, these values quantify the U.S. economy's annual contributions to each impact category. As such, they represent a "U.S. impact yardstick" against which to evaluate the significance of building-specific impacts. Normalization is accomplished by dividing BIRDS building-specific impact assessment results by the fixed U.S.-scale normalization references, expressed in the same units, yielding an impact category score for a building that has been placed in the context of annual U.S. contributions to that impact. By placing each building-specific impact result in the context of its associated U.S. impact result, the measures are all reduced to the same scale, allowing comparison across impacts.

\section{Table 5-3 BIRDS Normalization References}

\begin{tabular}{|c|c|c|}
\hline Impact Category & $\begin{array}{l}\text { Normalization reference } \\
\text { U.S. total/yr }\end{array}$ & Units \\
\hline Global Warming & $7.16 \mathrm{E}+12$ & $\mathrm{~kg}(\mathrm{lb}) \mathrm{CO}_{2} \mathrm{e}$ \\
\hline $\begin{array}{l}\text { Primary Energy } \\
\text { Consumption }\end{array}$ & $\begin{array}{c}3.52 \mathrm{E}+13 \\
(1.20 \mathrm{E}+14)\end{array}$ & $\begin{array}{l}\text { kWh } \\
\text { (kBTU) }\end{array}$ \\
\hline HH Criteria Air & $2.24 \mathrm{E}+10$ & $\begin{array}{l}\text { kg particulate matter } 10 \text { equivalents } \\
\text { (PM10 = particulate matter }<10 \text { microns in diameter) }\end{array}$ \\
\hline HH Cancer & $1.05 \mathrm{E}+04$ & comparative human toxicity units \\
\hline $\begin{array}{l}\text { Water } \\
\text { Consumption }\end{array}$ & $1.69 \mathrm{E}+14$ & $\begin{array}{l}\text { L } \\
\text { (gallon) }\end{array}$ \\
\hline Ecological Toxicity & $3.82 \mathrm{E}+13$ & comparative ecotoxicity units \\
\hline Eutrophication & $1.01 \mathrm{E}+10$ & kg (lb) nitrogen equivalents \\
\hline Land Use & $\begin{array}{c}7.32 \mathrm{E}+08 \\
(1.81 \mathrm{E}+09)\end{array}$ & $\begin{array}{l}\text { hectare } \\
\text { (acre) }\end{array}$ \\
\hline HH Non-cancer & $5.03 \mathrm{E}+05$ & comparative human toxicity units \\
\hline Smog Formation & $4.64 \mathrm{E}+11$ & kg (lb) ozone equivalents \\
\hline Acidification & $1.66 \mathrm{E}+12$ & moles hydrogen ion equivalents \\
\hline Ozone Depletion & $5.10 \mathrm{E}+07$ & $\begin{array}{l}\text { kg (lb) CFC-11 equivalents (CFC-11 = } \\
\text { trichlorofluoromethane) }\end{array}$ \\
\hline
\end{tabular}

The environmental impact of a single building is small relative to the total U.S. emissions in an impact category, leading to normalized values that are small fractions of a percent. To improve the user experience, the commercial database adjusted these normalized values by multiplying by the U.S. population ( 309 million as of 2010), creating a normalized value that represents the fraction of emissions per capita for each impact category.

\subsection{Life-cycle Interpretation}

At the BIRDS LCA interpretation step, a building's normalized impact values are evaluated. The midpoint-level impact assessment yields scores for twelve impact categories, making interpretation at this level difficult. To enable comparisons across buildings, the scores across 
impact categories may be synthesized. Note that in BIRDS, the synthesis of impact scores is optional.

Impact scores may be synthesized by weighting each impact category by its relative importance to overall environmental performance, then computing the weighted average impact score called its Environmental Impact Score (EIS). In the BIRDS software, the set of importance weights is selected by the user. Several alternative weight sets are provided as guidance, and may be either used directly or as a starting point for developing user-defined weights. The alternative weight sets are based on an EPA Science Advisory Board study, a BEES Stakeholder Panel's structured judgments, a set of equal weights, and a set exclusively focusing on the global warming impact, representing a spectrum of ways in which people value diverse aspects of the environment.

\subsubsection{EPA Science Advisory Board Study}

In 1990 and again in 2000, EPA's Science Advisory Board (SAB) developed lists of the relative importance of various environmental impacts to help EPA best allocate its resources (U.S. EPA Science Advisory Board 1990, U.S. EPA Science Advisory Board 2000). The following criteria were used to develop the lists:

- The spatial scale of the impact

- The severity of the hazard

- The degree of exposure

- The penalty for being wrong

Ten of the twelve BIRDS impact categories were covered by the SAB lists of relative importance:

- Highest-Risk Problems: global warming, land use

- High-Risk Problems: ecological toxicity, human health (cancer and non-cancer effects)

- Medium-Risk Problems: ozone depletion, smog, acidification, eutrophication, and human health - criteria air pollutants

The SAB did not explicitly consider primary energy consumption or water consumption. For BIRDS, these impacts are assumed to be relatively medium-risk and low-risk problems, respectively, based on other relative importance lists (Levin 1996).

Verbal importance rankings, such as "highest risk," may be translated into numerical importance weights by following ASTM International standard guidance for applying a Multi-attribute Decision Analysis method known as the Analytic Hierarchy Process (AHP) (ASTM 2011).

The AHP methodology suggests the following numerical comparison scale: 
1 Two impacts contribute equally to the objective (in this case environmental performance)

3 Experience and judgment slightly favor one impact over another

$5 \quad$ Experience and judgment strongly favor one impact over another

7 One impact is favored very strongly over another, its dominance demonstrated in practice

9 The evidence favoring one impact over another is of the highest possible order of affirmation

$* 2,4,6$, and 8 can be selected when compromise between values of $1,3,5,7$, and 9 , is needed.

Through an AHP known as pairwise comparison, numerical comparison values are assigned to each possible pair of environmental impacts. Relative importance weights can then be derived by computing the normalized eigenvector of the largest eigenvalue of the matrix of pairwise comparison values. Table 5-4 and Table 5-5 list the pairwise comparison values assigned to the verbal importance rankings, and the resulting SAB importance weights computed for the BIRDS impacts, respectively. Note that the pairwise comparison values were assigned through an iterative process based on NIST's background and experience in applying the AHP technique. Furthermore, while the SAB evaluated cancer and non-cancer effects as a group, the resulting $13 \%$ weight was apportioned between the two based on the relative judgments of the BEES Stakeholder Panel discussed in the next section.

Table 5-4 Pairwise Comparison Values for Deriving Impact Category Importance Weights

\begin{tabular}{|lc|}
\hline Verbal Importance Comparison & Pairwise Comparison Value \\
\hline Highest vs. Low & 6 \\
\hline Highest vs. Medium & 3 \\
\hline Highest vs. High & 1.5 \\
\hline High vs. Low & 4 \\
\hline High vs. Medium & 2 \\
\hline Medium vs. Low & 2 \\
\hline
\end{tabular}

Table 5-5 Relative Importance Weights based on Science Advisory Board Study

\begin{tabular}{|c|c|}
\hline Impact Category & Relative Importance Weight (\%) \\
\hline Global Warming & 18 \\
\hline Primary Energy Consumption & 7 \\
\hline HH Criteria Air & 7 \\
\hline HH Cancer & 8 \\
\hline Water Consumption & 3 \\
\hline Ecological Toxicity & 12 \\
\hline Eutrophication & 5 \\
\hline Land Use & 18 \\
\hline HH Non-cancer & 5 \\
\hline Smog Formation & 7 \\
\hline Acidification & 5 \\
\hline Ozone Depletion & 5 \\
\hline
\end{tabular}

The EPA SAB weights should be updated in the future when updated qualitative environmental impact importance comparisons are available from the EPA. 


\subsubsection{BEES Stakeholder Panel Judgments}

While the derived EPA SAB-based weight set is helpful and offers expert guidance, several interpretations and assumptions were required in order to translate SAB findings into numerical weights for interpreting LCA-based analyses. A more direct approach to weight development would consider a closer match to the context of the application; that is, environmentally preferable purchasing in the United States based on life-cycle impact assessment results, as reported by BIRDS.

In order to develop such a weight set, NIST assembled a volunteer stakeholder panel that met at its facilities in Gaithersburg, Maryland, for a full day in May 2006. To convene the panel, invitations were sent to individuals representing one of three "voting interests:" producers (e.g., building product manufacturers), users (e.g., green building designers), and LCA experts. Nineteen individuals participated in the panel: seven producers, seven users, and five LCA experts. These "voting interests" were adapted from the groupings ASTM International employs for developing voluntary standards, in order to promote balance and support a consensus process.

The BEES Stakeholder Panel was led by Dr. Ernest Forman, founder of the AHP firm Expert Choice Inc. Dr. Forman facilitated panelists in weighting the BEES impact categories using the AHP pairwise comparison process. The panel weighted all impacts in the Short Term (0 years to 10 years), Medium Term (10 years to 100 years), and Long Term (>100 years). One year's worth of U.S. flows for each pair of impacts was compared, with respect to their contributions to environmental performance. For example, for an impact comparison over the Long Term, the panel evaluated the effect that the current year's U.S. emissions would have more than 100 years hence.

Once the panel pairwise-compared impacts for the three time horizons, its judgments were synthesized across the selected time horizons. Note that when synthesizing judgments across voting interests and time horizons, all panelists were assigned equal importance, while the short, medium, and long-term time horizons were assigned by the panel to carry $24 \%$, $31 \%$, and $45 \%$ of the weight, respectively.

The environmental impact importance weights developed through application of the AHP technique at the facilitated BEES Stakeholder Panel event are shown in Table 5-6. These weights reflect a synthesis of panelists' perspectives across all combinations of stakeholder voting interest and time horizon. The weight set draws on each panelist's personal and professional understanding of, and value attributed to, each impact category. While the synthesized weight set may not equally satisfy each panelist's view of impact importance, it does reflect contemporary values in applying LCA to real world decisions. This synthesized BEES Stakeholder Panel weight set is offered as an option in BIRDS online.

The panel's application of the AHP process to derive environmental impact importance weights is documented in an appendix to Gloria, Lippiatt et al. (2007) and ASTM (2011). 
Table 5-6 Relative Importance Weights based on BEES Stakeholder Panel Judgments

\begin{tabular}{|l|r|}
\hline Impact Category & Relative Importance Weight (\%) \\
\hline Global Warming & 29.9 \\
\hline Primary Energy Consumption & 10.3 \\
\hline HH Criteria Air & 9.3 \\
\hline HH Cancer & 8.2 \\
\hline Water Consumption & 8.2 \\
\hline Ecological Toxicity & 7.2 \\
\hline Eutrophication & 6.2 \\
\hline Land Use & 6.2 \\
\hline HH Non-cancer & 5.2 \\
\hline Smog Formation & 4.1 \\
\hline Acidification & 3.1 \\
\hline Ozone Depletion & 2.1 \\
\hline Note: Sin BIRD & \\
\hline
\end{tabular}

Note: Since BIRDS does not currently include an Indoor Air

Quality impact category, its 3 \% BEES Stakeholder Panel weight

has been redistributed by proportion among the remaining 12

impacts.

The three figures below display in graphical form the BEES Stakeholder Panel weights used in BIRDS. Figure 5-3 displays the synthesized weight set. Figure 5-4 displays the weights specific to panelist voting interest, and Figure 5-5 displays the weights specific to time horizon. The BIRDS user is free to interpret results using either of the weight sets displayed in Figure 5-4 and Figure 5-5 by entering them as a user-defined weight set. It would be beneficial to convene a new stakeholder panel to realign these environmental weights with current stakeholder consensus.

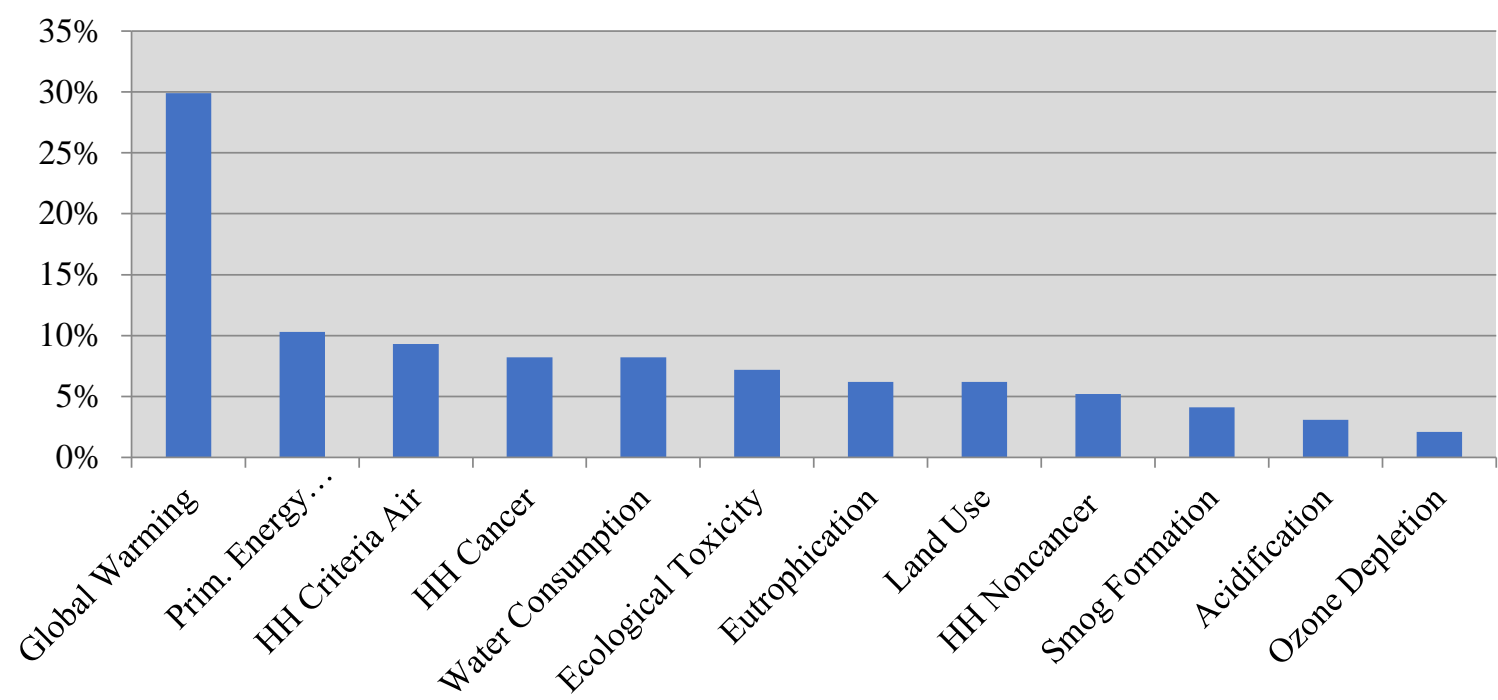

Impact Category

Figure 5-3 BEES Stakeholder Panel Importance Weights Synthesized across Voting Interest and Time Horizon 


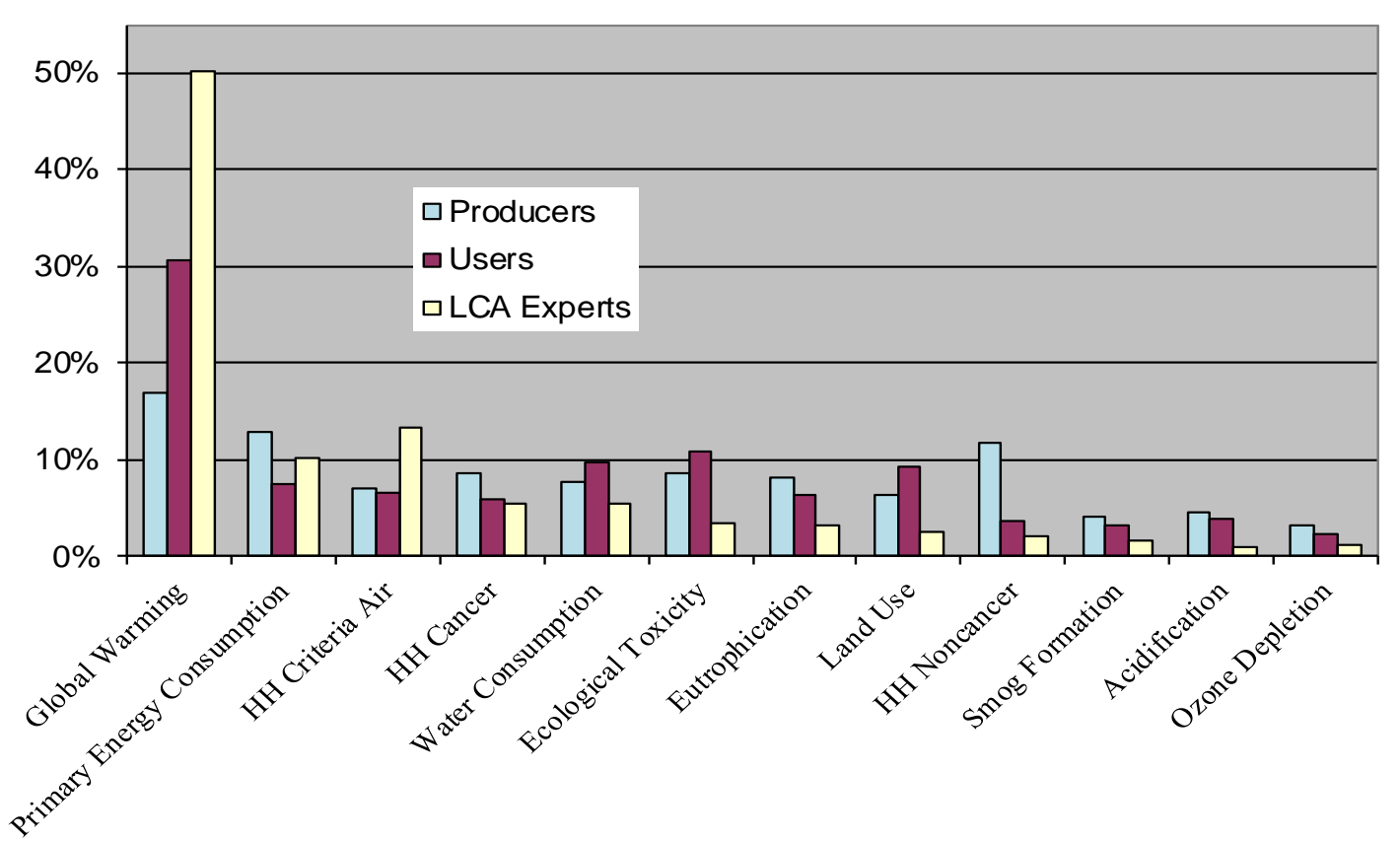

Figure 5-4 BEES Stakeholder Panel Importance Weights by Stakeholder Voting Interest

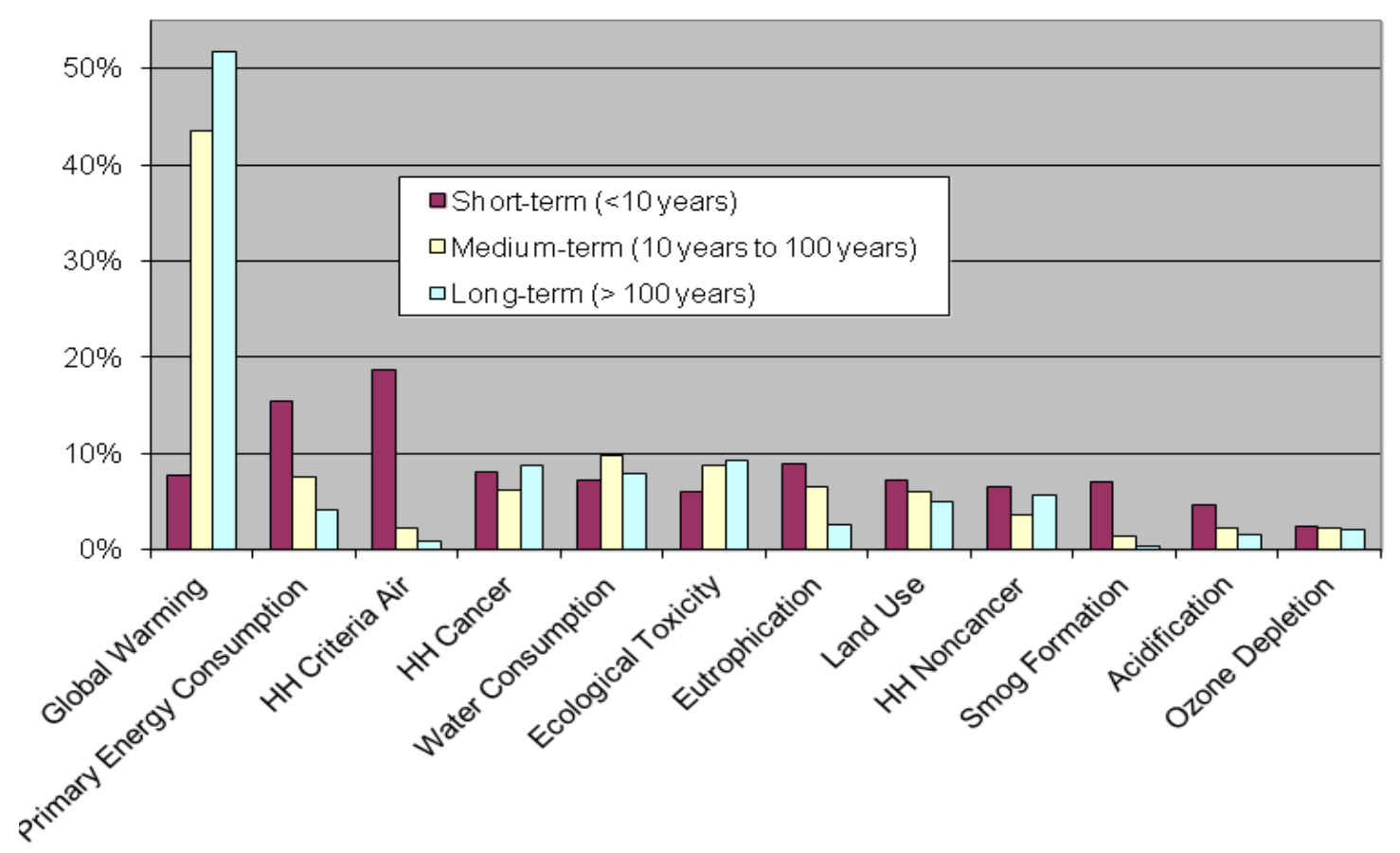

Figure 5-5 BEES Stakeholder Panel Importance Weights by Time Horizon

\subsection{BIRDS Energy Technologies}

Since buildings are operated for longer than the maximum study period of 40 years, operating energy efficiency has an important influence on their sustainability performance. Energy 
efficiency is largely driven by a building's energy technologies, but top-down inventory data are not readily available at this level of resolution. Therefore, BIRDS includes detailed LCI data for a range of energy technology packages that have been compiled at the traditional, bottom-up LCA level. These energy technology packages are used to match the 13680 building prototype-location-standard edition combinations in the commercial database. The bottom-up BIRDS data were developed under contract to NIST by Four Elements Consulting, LLC, of Seattle, Washington. Energy technologies include wall and ceiling insulation, windows, HVAC equipment, lighting, and interior and exterior sealants.

\subsubsection{General Information Regarding the Energy Technology LCIs}

\subsubsection{Standards Used}

The LCAs in BIRDS have been built based on the principles and framework in the International Organization for Standardization (ISO) (2006a) and the guidelines specified in International Organization for Standardization (ISO) (2006b).

\subsubsection{Primary and Secondary Data Sources}

Both primary data (collected directly from a manufacturing facility) and secondary data (publicly-available literature sources) can be used to build LCAs, and it is common to see a combination of both data types based on the level of disclosure organizations or companies prefer related to the information pertaining to their products. Sources of data on the energy technologies in BIRDS vary from one category to the next, and within categories themselves for the different products. Data were based on one or more of the following:

- Primary data from a group of companies and/or an industry association, compiled into an industry average product;

- Primary data on a product provided by one company;

- Secondary data that represent an average or typical product; and/or

- Secondary data that represent one product in a category.

For optimal data quality, the preference is to have the most representative data - temporally, technologically, and geographically - on a product or system, so that the model produced most closely represents the product. But this is often not possible to achieve due to data constraints. It is also not always possible to have a data set that represents an entire category of products. For example, high quality, current, company-specific data might be collected and used to build the LCA for a given product. Likewise, data for another product might be compiled from literature sources due to lack of other available data. In both cases, the LCI profiles may be used to represent the full product category, even though they may not be representative of all products within the category, based on market share, technology, geographical location, etc. The user should be aware of this limitation. 


\subsubsection{Data Sources Used for the Background Data}

Secondary data have been applied to production of material inputs, production and combustion of fuels used for process energy, and transportation processes. The U.S. LCI Database (National Renewable Energy Laboratory (NREL) 2012) and the ecoinvent v.2.2 database (Ecoinvent 2007) are the main sources of background data throughout the various life-cycle stages. Other sources of data were used where data were not available from the U.S. LCI Database or ecoinvent, and/or where they were deemed to be of better quality than these sources.

The following subsections describe modeling, assumptions, and data sources of the product life-cycle data. Data for material inputs production for each product are also described as these may vary for different industries. The following data aspects are consistent for all products except where noted differently in the subchapters:

- All energy production, including production of fuels and conversion into energy and electricity production come from the U.S. LCI Database.

- All transportation data come from the U.S. LCI Database.

- Whenever possible, where ecoinvent or other non-North American data sets were used, they were customized into North American processes by switching out foreign energy, electricity, transportation, and other processes for comparable North American based data sets from the U.S. LCI databases. Exceptions to this are noted.

- Data for parts forming (e.g., forming of metal parts and pieces, injection molding of plastic parts, etc.), are modeled with raw materials production. Most of these data come from ecoinvent.

\subsubsection{Wall, Roof, and Foundation Insulation}

The insulation categories considered for the commercial building walls, roofs, and foundations are presented in Table 5-7, Table 5-8, and Table 5-10 along with the R-values needed to meet the necessary thickness of insulation products to meet the requirements across all climate zones and editions of ASHRAE 90.1. Characteristics of each insulation type, including density and R-values $\left(\frac{m^{2} * K}{W}\right.$ per cm or $\frac{f t^{2} * K * h}{B t u}$ per inch) are presented in the specific products' subsections. 
Table 5-7 Specified Insulation Types and R-Values - Wall Application

\begin{tabular}{|c|c|c|}
\hline $\begin{array}{l}\text { Building } \\
\text { Type }\end{array}$ & & Insulation Levels \\
\hline \multirow[t]{2}{*}{$\begin{array}{l}\text { Metal } \\
\text { Building }\end{array}$} & Rigid & $\begin{array}{l}\text { R-0+R-15.8 c.i. } \\
\text { R-0+R-19 c.i. } \\
\text { R-0+R-22.1 c.i. } \\
\text { R-0+R-25 c.i. } \\
\text { R-0+R-9.8 c.i. }\end{array}$ \\
\hline & $\begin{array}{l}\text { Fiberglass } \\
\text { Batt + Rigid } \\
\text { - Polyiso }\end{array}$ & $\begin{array}{l}\text { R-13.0 } \\
\text { R-13.0+R-13.0 } \\
\text { R-13+R-5.6 c.i. } \\
\text { R-16 } \\
\text { R-19 } \\
\text { R-19+R-5.6 c.i. }\end{array}$ \\
\hline Wood-Framed & $\begin{array}{l}\text { Fiberglass } \\
\text { Batt + Rigid } \\
\text { Polyiso }\end{array}$ & $\begin{array}{l}\text { R-13 } \\
\text { R-13.0+R-3.8 c.i. } \\
\text { R-13.0+R-7.5 c.i. } \\
\text { R-13+R-10 c.i. } \\
\text { R-13+R-12.5 c.i. } \\
\text { R-13+R-15.6 c.i. } \\
\text { R-13+R-18.8 c.i. }\end{array}$ \\
\hline \multicolumn{3}{|c|}{ Note: $\mathrm{R}-1=\mathrm{R}_{\mathrm{SI}}-0.18$} \\
\hline
\end{tabular}

\begin{tabular}{|l|l|l|}
\hline $\begin{array}{l}\text { Building } \\
\text { Type }\end{array}$ & & Insulation Levels \\
\hline Steel-Framed & Fiberglass Batt + & R-13 \\
& Rigid - Polyiso & R-13+R-3.8 c.i. \\
& & R-13+R-5 c.i. \\
& & R-13+R-7.5 c.i. \\
& & R-13+R-10 c.i. \\
& & R-13+R-12.5 c.i. \\
& & R-13+R-15.6 c.i. \\
& & R-13+R-18.8 c.i. \\
\hline Mass-CME & Rigid - Polyiso & R-11.4 c.i. \\
& & R-13.3 c.i. \\
& & R-15.2 c.i. \\
& & R-19 c.i. \\
& & R-5.7 c.i. \\
& & R-7.6 c.i. \\
\hline Note: R-1 = R & & R-9.5 c.i. \\
\hline
\end{tabular}

Table 5-8 Specified Insulation Types and R-Values - Roof Application

\begin{tabular}{|c|c|c|}
\hline $\begin{array}{l}\text { Construction } \\
\text { Type }\end{array}$ & $\begin{array}{l}\text { Insulation } \\
\text { Type } \\
\end{array}$ & R-Value \\
\hline \multirow{4}{*}{$\begin{array}{l}\text { Attic and } \\
\text { Other }\end{array}$} & \multirow{4}{*}{$\begin{array}{l}\text { Fiberglass } \\
\text { Batt }\end{array}$} & $\mathrm{R}-30$ \\
\hline & & $\mathrm{R}-38$ \\
\hline & & $\mathrm{R}-49$ \\
\hline & & $\mathrm{R}-60$ \\
\hline \multirow[t]{5}{*}{ IEAD } & \multirow{5}{*}{$\begin{array}{l}\text { Rigid - } \\
\text { XPS }\end{array}$} & R-15 c.i. \\
\hline & & R-20 c.i. \\
\hline & & R-25 c.i. \\
\hline & & R-30 c.i. \\
\hline & & R-35 c.i. \\
\hline
\end{tabular}

\begin{tabular}{|c|c|c|}
\hline $\begin{array}{l}\text { Construction } \\
\text { Type } \\
\end{array}$ & $\begin{array}{l}\text { Insulation } \\
\text { Type }\end{array}$ & R-Value \\
\hline \multirow{10}{*}{$\begin{array}{l}\text { Metal } \\
\text { Building }\end{array}$} & \multirow{10}{*}{$\begin{array}{l}\text { Fiberglass Batts } \\
\text { (Cavity + } \\
\text { Draped) }\end{array}$} & $\mathrm{R}-10+\mathrm{R}-19 \mathrm{FC}$ \\
\hline & & $\mathrm{R}-11+\mathrm{R}-19 \mathrm{Ls}$ \\
\hline & & $\mathrm{R}-13.0+\mathrm{R}-19.0$ \\
\hline & & $\mathrm{R}-13+\mathrm{R}-13$ \\
\hline & & $\mathrm{R}-13+\mathrm{R}-19$ \\
\hline & & $\mathrm{R}-19$ \\
\hline & & R-19+R-11 Ls or R-25+R-8 Ls \\
\hline & & R-25+R-11 Ls \\
\hline & & $\mathrm{R}-25+\mathrm{R} 11+\mathrm{R}-11 \mathrm{Ls}$ \\
\hline & & R-30+R-11 Ls \\
\hline \multicolumn{3}{|c|}{ Note: $\mathrm{R}-1=\mathrm{R}_{\mathrm{SI}}-0.18$} \\
\hline
\end{tabular}


Table 5-9 Specified Insulation Types and R-Values - Foundation Application

\begin{tabular}{lccc} 
Adjustment & Slab Insulation R-Value & Slab Insulation Depth & Wall Insulation \\
\hline Basement & $\mathrm{NR}$ & $\mathrm{NR}$ & $\mathrm{NR}$ \\
& $\mathrm{NR}$ & $\mathrm{NR}$ & $\mathrm{R}-7.5$ c.i. \\
& $\mathrm{NR}$ & $\mathrm{NR}$ & $\mathrm{R}-10.0$ c.i. \\
$\mathrm{NR}$ & $\mathrm{NR}$ & $\mathrm{R}-15.0$ c.i. \\
\hline Slab on Grade & $\mathrm{R}-5$ & 24 in & N/A \\
& $\mathrm{R}-10$ & $48 \mathrm{in}$ & $\mathrm{N} / \mathrm{A}$ \\
& $\mathrm{R}-15$ & $48 \mathrm{in}$ & $\mathrm{N} / \mathrm{A}$ \\
$\mathrm{R}-20$ & $48 \mathrm{in}$ & $\mathrm{N} / \mathrm{A}$ \\
$\mathrm{R}-25$ & $48 \mathrm{in}$ & $\mathrm{N} / \mathrm{A}$ \\
\hline Note: $\mathrm{R}-1=\mathrm{R}_{\mathrm{SI}}-0.18 ; \mathrm{NR}=$ No Requirement; N/A = Not Applicable \\
\hline
\end{tabular}

BIRDS performance data for the insulation category was provided based on $0.09 \mathrm{~m}^{2}\left(1 \mathrm{ft}^{2}\right)$ of the specified R-value of insulation, which was then multiplied by the needed amount of square area for each building. Figure 5-6 presents the general system boundaries for the insulation category as it is modeled for BIRDS.

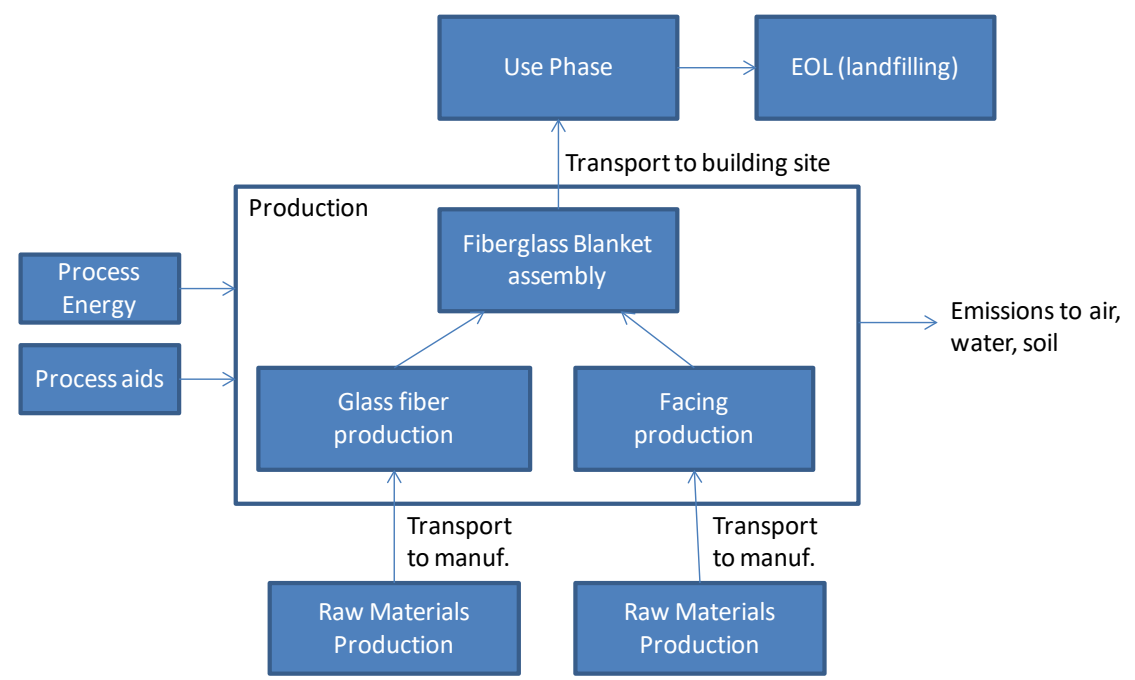

Figure 5-6 Insulation System Boundaries - Fiberglass Blanket Example

The modeling and assumptions for each type of insulation are presented in the following sections.

\subsubsection{Fiberglass Blanket}

Fiberglass blanket, or batt, insulation is made by forming spun-glass fibers into batts. At an insulation plant, the product feedstock is weighed and sent to a melting furnace. The raw materials are melted in a furnace at very high temperatures. Streams of the resulting vitreous melt are either spun into fibers after falling onto rapidly rotating flywheels or drawn through tiny holes in rapidly rotating spinners. This process shapes the melt into fibers. Glass coatings are added to the fibers that are then collected on conveyers. The structure and density of the product is continually controlled by the conveyer speed and height as it passes through a curing oven. 
The cured product is then sawn or cut to the required size. Off-cuts and other scrap material are recycled back into the production process.

Thermal resistance values of $\mathrm{R}_{\mathrm{SI}}-2.3$ (R-11), $\mathrm{R}_{\mathrm{SI}}-2.3$ (R-13), $\mathrm{R}_{\mathrm{sI}}-2.3$ (R-15), and $\mathrm{R}_{\mathrm{SI}}-3.4$ (R-19) are used for wall insulation and specifies fiberglass insulation by type and R-value. Most of the fiberglass insulation data is based on the model presented in the online documentation of the BEES software (NIST 2010). LCA estimates for products not listed are extrapolated from the most comparable product.

Table 5-10 Fiberglass Blanket Mass by Application

\begin{tabular}{|c|c|c|c|c|c|}
\hline Application & $\begin{array}{c}\text { Thickness } \\
\text { cm (in) }\end{array}$ & $\begin{array}{l}\text { Density } \mathbf{k g} / \mathrm{m}^{3} \\
\left(\mathrm{lb} / \mathrm{ft}^{3}\right)\end{array}$ & $\begin{array}{l}\text { Mass per } 1 \text { in } \\
\text { Functional Unit } \\
\mathbf{k g} / \mathbf{m}^{2}\left(\mathrm{oz} / \mathbf{f t}^{2}\right)\end{array}$ & $\begin{array}{c}\text { R-Value per } \\
\text { Reference Flow } \\
\left(\mathbf{m}^{2} \mathrm{~K} / \mathrm{W} \text { per } 1 \mathrm{~cm}\right)\end{array}$ & $\begin{array}{c}\text { R-Value per } \\
\text { Reference Flow } \\
\left(\mathrm{ft}^{2}{ }^{\circ} \mathrm{F} \mathrm{hr} / \text { Btu per } 1 \text { in) }\right.\end{array}$ \\
\hline $\begin{array}{l}\text { Wall- } \mathrm{R}_{\mathrm{SI}}-1.9 \\
(\mathrm{R}-11)\end{array}$ & $8.9(3.5)$ & $12.1(0.76)$ & $1.07(3.52)$ & & \\
\hline $\begin{array}{l}\text { Wall - R } \mathrm{R}_{\mathrm{SI}}-2.3 \\
(\mathrm{R}-13)\end{array}$ & $8.9(3.5)$ & $12.1(0.76)$ & $0.31(1.01)$ & $\mathrm{R}-0.26$ & R-3.7 \\
\hline $\begin{array}{l}\text { Wall- } \mathrm{R}_{\mathrm{SI}}-2.6 \\
(\mathrm{R}-15)\end{array}$ & 8.9 (3.5) & $22.6(1.41)$ & $2.01(6.58)$ & & \\
\hline $\begin{array}{l}\text { Wall - R } \mathrm{R}_{\mathrm{SI}}-3.4 \\
(\mathrm{R}-19)\end{array}$ & $15.9(6.25)$ & $7.0(0.44)$ & $0.18(0.58)$ & R-0.21 & R-3.0 \\
\hline
\end{tabular}

\subsection{Upstream Materials Production through Manufacturing}

Fiberglass insulation is made with a blend of sand, limestone, soda ash, and recycled glass cullet. Recycled window, automotive, or bottle glass is used in the manufacture of glass fiber; it accounts for $30 \%$ to $50 \%$ of the raw material input. The recycled content is limited by the amount of usable recycled material available in the market - not all glass cullet is of sufficient quality to be used in the glass fiber manufacturing process. The use of recycled material has helped to steadily reduce the energy required to produce insulation products. The raw materials used to produce fiberglass insulation are broken down by the glass and facing contents, shown in Table 5-11.

Table 5-11 Fiberglass Insulation Constituents

\begin{tabular}{|lc|}
\hline Glass Constituent & Mass Fraction (\%) \\
\hline Soda Ash & 9.0 \\
\hline Borax & 12.0 \\
\hline Glass Cullet & 34.0 \\
\hline Limestone & 9.0 \\
\hline Phenolic resin (binder coating) & 5.0 \\
\hline Sand & 31.0 \\
\hline Total & $\mathbf{1 0 0}$ \\
\hline Facing & Mass Fraction (\%) \\
\hline Kraft paper & 25.0 \\
\hline Asphalt & 75.0 \\
\hline Total & $\mathbf{1 0 0}$ \\
\hline
\end{tabular}


The production data for the soda ash, limestone, and phenol formaldehyde resin come from the U.S. LCI Database. The borax, glass cullet, and silica sand data come from ecoinvent. For the facing, Kraft paper data come from ecoinvent and the asphalt data come from U.S. LCI Database.

The raw materials are transported to the manufacturing plant via diesel truck. Materials are sourced domestically, and transportation distances range on average from $161 \mathrm{~km}(100 \mathrm{mi})$ to 805 km (500 mi).

The energy requirements for melting the glass constituents into fibers and drying off the completed blanket involve use of natural gas and electricity, shown in Table 5-12.

Table 5-12 Energy Requirements for Fiberglass Insulation Manufacturing

\begin{tabular}{lc} 
Energy Carrier & MJ/kg (Btu/lb) \\
Natural Gas & $1.99(857)$ \\
Electricity & $1.37(591)$ \\
Total & $\mathbf{3 . 3 6}(\mathbf{1 4 4 8 )}$ \\
\hline
\end{tabular}

Besides combustion emissions from fuel usage at manufacturing, particulates are emitted at a rate of $2.38 \mathrm{~g} / \mathrm{kg}$ ( $4.76 \mathrm{lb} / \mathrm{ton}$ ) of bonded blankets and volatile organic compounds (VOCs) are emitted at a rate of $0.76 \mathrm{~g} / \mathrm{kg}(1.52 \mathrm{lb} / \mathrm{ton})$ of bonded blankets.

All waste produced during the cutting and blending process is either recycled into other insulation materials or added back into the glass mix. Thus, no solid waste is assumed to be generated during the production process.

\subsection{Transportation to the Building through End-of-Life}

Transportation of the insulation from the manufacturer to the building site is assumed to be an average of $805 \mathrm{~km}$ (500 mi) by heavy-duty diesel-fueled truck.

Installing fiberglass blanket insulation is primarily a manual process, with no energy or emissions included in the model. During installation, any waste material is added into the building shell where the insulation is installed - there is effectively no installation waste.

Fiberglass insulation has a functional lifetime of over 50 years, so no replacement is needed during the 40-year study period. How this product affects operating energy during the home's use phase is addressed in other sections of this report.

While fiberglass insulation is recyclable, it is assumed that it is disposed of in a landfill at endof-life. End-of-life modeling includes transportation by heavy-duty diesel-fuel powered truck approximately $80 \mathrm{~km}$ (50 mi) to a construction and demolition (C\&D) landfill. Landfilled insulation is modeled based on ecoinvent end-of-life waste management process data. 


\subsubsection{XPS Foam Insulation}

Extruded Polystyrene (XPS) foam insulation has been modeled for the commercial exterior wall. Type IV and Type XPS may be used for residential applications; the data in this document is representative of Type IV XPS, which has a typical average density of $26.2 \mathrm{~kg} / \mathrm{m}^{3}\left(1.63 \mathrm{lb} / \mathrm{ft}^{3}\right)$. The foam has the following R-values, depending on thickness: ${ }^{9}$

- $2.5 \mathrm{~cm}(1 \mathrm{in}): \mathrm{R}_{\mathrm{SI}}=0.9(\mathrm{R}=5.0)$

- $5.1 \mathrm{~cm}(2 \mathrm{in}): \mathrm{R}_{\mathrm{SI}}=1.9(\mathrm{R}=10.6)$

- $7.6 \mathrm{~cm}(3 \mathrm{in}): \mathrm{R}_{\mathrm{SI}}=2.9(\mathrm{R}=16.2)$

- $10.2 \mathrm{~cm}(4 \mathrm{in}): \mathrm{R}_{\mathrm{SI}}=3.9(\mathrm{R}=22.0)$

\subsection{Upstream Materials Production through Manufacturing}

Extruded Polystyrene Foam Association (XPSA) member companies provided representative industry average production data on XPS foam boards. XPSA represents the three largest producers in North America and accounts for over $95 \%$ of XPS products produced and sold. Table 5-13 provides a 2010 representative average of the raw material and processing energy inputs and process outputs to produce one $\mathrm{kg}$ of XPS foam board.

The table presents the current representative blowing agent assumptions for XPS. It should be noted that HFCs began to replace 1-Chloro-1, 1-difluoroethane (HCFC-142b) as the principal blowing agent in 2009, as the industry complied with U.S. EPA and Environment Canada ODS phase-out regulations requiring the XPS sector to discontinue HCFC use by the end of 2009. By 2010, all XPSA members had converted to non-HCFC blowing agents and have been using only HFC materials ever since.

Table 5-13 XPS Foam Board Production Data

\begin{tabular}{|lllc|}
\hline Inputs & & Unit & Quantity per kg (per lb) \\
\hline Blowing agents & HFC-134a & $\mathrm{kg}(\mathrm{lb})$ & $0.060(0.060)$ \\
\hline & HFC-152a & $\mathrm{kg}(\mathrm{lb})$ & $0.017(0.017)$ \\
\hline & $\mathrm{CO}_{2}$ & $\mathrm{~kg}(\mathrm{lb})$ & $0.012(0.012)$ \\
\hline Solid additives & PS resin & $\mathrm{kg}(\mathrm{lb})$ & $0.907(0.907)$ \\
\hline & Additives & $\mathrm{kg}(\mathrm{lb})$ & $0.018(0.018)$ \\
\hline Energy & Electricity & $\mathrm{kWh}$ & $1.00(0.454)$ \\
\hline Outputs & & & \\
\hline Air & HFC-134a & $\mathrm{kg}(\mathrm{lb})$ & $0.0105(0.0105)$ \\
\hline & HFC-152a & $\mathrm{kg}(\mathrm{lb})$ & $0.0029(0.0029)$ \\
\hline Waste & Waste & $\mathrm{kg}(\mathrm{lb})$ & $1.0 \mathrm{E}-4(1.0 \mathrm{E}-4)$ \\
\hline
\end{tabular}

\footnotetext{
${ }^{9}$ Extruded Polystyrene Foam Association (XPSA) website, found at http://www.xpsa.com. Values are based on a round-robin study in 2003 using the CAN/ULC S770-00 LTTR standard.
} 
The additives in the table include the flame retardant widely used in all polystyrene foams (hexabromocyclododecane (HBCD)) and colorants or dyes/pigments used to produce the characteristic color of each XPSA member's foam. Additives may also include a nucleation control agent, process lubricant, acid scavenger, or others.

The blowing agent conversion/trim losses during manufacturing are assumed to be on average $17.5 \%$ for North American XPS foam production (Intergovernmental Panel on Climate Change (IPCC) 2005). All polystyrene trim waste at the manufacturing plant is reused internally in the process. Only a very small amount of foam and other materials are occasionally sent off-site for disposal in a landfill.

Data for polystyrene come from the U.S. LCI Database. Data for all three blowing agents and some of the additives come from ecoinvent. Data were not available for all of the additives; where data were not available proxy data were implemented.

All the raw materials are produced in the U.S., and most of the raw materials are centrally located, as are the XPS manufacturer's largest plants. The estimated weighted average distance from the main suppliers to most XPS manufacturing plants is $805 \mathrm{~km}$ (500 mi) for polystyrene, Tetrafluoroethane (HFC-134a), flame retardant, and $\mathrm{CO}_{2}$. 1,1-Difluoroethane (HFC-152a) and other additives are transported an average distance of $1609 \mathrm{~km}$ (1000 mi) to manufacturing plants. All but the blowing agents and polystyrene are transported by diesel truck; the blowing agents and polystyrene are transported by rail.

\subsection{Transportation to the Building Site through End-of-Life}

Transportation of the insulation to the building site is modeled as $563 \mathrm{~km}$ (350 mi), an average factoring in the various plants around the United States. Transportation is by heavy-duty diesel truck.

Foam boards are installed with installation tape, but tape is excluded since it is considered negligible. Scrap XPS foam board generated at installation is assumed to be $2 \%$ of the total, consistent with other foam products in this category. While the product may be recyclable, it is modeled as being sent to a landfill $32 \mathrm{~km}(20 \mathrm{mi})$ from the building site. Data for the landfill come from waste management datasets in ecoinvent. Blowing agent escape during installation is insignificant. Minimal cutting to size on the jobsite is done and, even then, a sharp tool is typically used so that very few cells are opened.

XPS insulation has a functional lifetime of over 40 years so no replacement is needed during the 40 -year study period. How insulation in the buildings affects operating energy during the prototype buildings' use phase is addressed in Chapter 0.

The diffusion of HFC-134a from XPS during use is $0.75 \%+/-0.25 \%$ per (Intergovernmental Panel on Climate Change (IPCC) 2005). The blowing agent emission loss during the use phase is complex and non-linear but can be represented for simplicity as a linear function after the first year. The rate is a function of the product thickness, properties (density, cell size, skins), blowing agent type(s) and transport properties (solubility, diffusion coefficient), and the installed 
application details (mean temperature, permeability of applied facings). The diffusion rate of HFC-152a is 15 \% per year (Intergovernmental Panel on Climate Change (IPCC) 2005).

Reuse of the foam is possible after building decommissioning, but the model assumes that at end-of-life the foam is disposed of in a landfill. End-of-life modeling includes transportation by heavy-duty diesel-fuel powered truck approximately $80 \mathrm{~km}$ (50 mi) to a C\&D landfill. Landfilled insulation is modeled based on ecoinvent end-of-life waste management process data. For a typical North America building demolition followed by disposal in a landfill, it is reasonable to assume an initial blowing agent end-of-life loss of $20 \%$ followed by annual losses of $1 \%$ (United Nations Environment Programme (UNEP) 2005).

\subsubsection{Polyisocyanurate Foam Insulation}

Polyisocyanurate (polyiso) foam insulation has been modeled for the exterior wall application. The thermal resistance value for wall polyiso board is 6.5 which includes its impermeable board facer. This R-value is based on a 6-month accelerated aging test and was provided by representatives at Bayer MaterialScience ${ }^{10}$. The foam has a wet, or pre-yield, density of $29.2 \mathrm{~kg} / \mathrm{m}^{3}\left(1.82 \mathrm{lb} / \mathrm{ft}^{3}\right)$. The final product, which includes the weight of the facers, has a nominal density of $32.0 \mathrm{~kg} / \mathrm{m}^{3}\left(2.0 \mathrm{lb} / \mathrm{ft}^{3}\right)$.

\subsection{Upstream Materials Production through Manufacturing Upstream Materials Production}

Cradle-to-gate data on production through manufacturing is based on a 2010 study performed for the Polyisocyanurate Insulation Manufacturers Association (PIMA) (Bayer MaterialScience 2008). The scope of this study included collecting and compiling mostly 2007 production data from the six PIMA member companies and compiling it into an industry average polyiso insulation board. Process energy data came from 29 out of 31 polyiso plants in the U.S. and Canada, representing approximately $94 \%$ of production in those geographies.

The chemicals to produce polyiso foam make up an "A" side (MDI) and a "B" side (polyester polyol with various additives such as catalysts, surfactants and flame retardants) plus a blowing agent (pentane). Table 5-14 presents the raw material inputs associated with polyiso foam production (Bayer MaterialScience 2011), provided on the basis of $2.54 \mathrm{~cm}$ (1 in) in thickness.

${ }^{10}$ Verbal communication with Bayer MaterialScience representatives, July 2013. 
Table 5-14 Raw Material Inputs to Produce Polyiso Foam

\begin{tabular}{|lccc|}
\hline Inputs & \% in foam & $\begin{array}{c}\mathbf{k g ~ p e r ~} \mathbf{0 . 0 9} \mathbf{~ m}^{\mathbf{2}}, \\
\mathbf{2 . 5 4} \mathbf{~ c m ~ t h i c k}\end{array}$ & $\begin{array}{c}\mathbf{l b} \text { per } \mathbf{~ f t}{ }^{2} \\
\mathbf{1} \text { in thick }\end{array}$ \\
\hline MDI & 55.5 & 0.0382 & 0.0842 \\
\hline Polyester Polyol & 31 & 0.0213 & 0.0470 \\
\hline TCPP & 3.4 & 0.0023 & 0.0051 \\
\hline Catalyst K15 & 1.4 & 0.0010 & 0.0022 \\
\hline Catalyst PC46 & 0.16 & $1.38 \mathrm{E}-04$ & 0.0003 \\
\hline Catalyst PV & 0.08 & $6.90 \mathrm{E}-05$ & 0.0002 \\
\hline Surfactant & 0.63 & $5.51 \mathrm{E}-04$ & 0.0012 \\
\hline Pentane (blowing agent) & 7.5 & 0.0052 & 0.0115 \\
\hline Process water & 0.1 & 0.0001 & 0.0002 \\
\hline
\end{tabular}

The MDI data come from the U.S. LCI Database. Data for the polyester polyol comes from an eco-profile study of aromatic polyester polyols (PE International 2010). Data for TCPP are U.S. data compiled from literature sources (PE International 2011). Pentane data come from ecoinvent. No data were available to include the three catalysts or silicone surfactant; they total $2.3 \%$ of the total input, so a total of $97.7 \%$ of the inputs were included in the model.

Polyiso wall applications normally use aluminum Kraft paper (foil) for the facer. The foil facer raw materials include paper, aluminum foil, adhesives and coatings, and has a mass of 0.098 $\mathrm{kg} / \mathrm{m}^{2}\left(0.02 \mathrm{lb} / \mathrm{ft}^{2}\right)$ (Bayer MaterialScience 2008). Data on material composition come from a material safety data sheet (MSDS). Based on this limited data source, the facer is modeled as 77 \% foil and 23 \% Kraft (Atlas Roofing Corporation 2012). Data for foil is modeled as 50/50 primary and secondary aluminum from the U.S. LCI Database, plus sheet rolling (ecoinvent). Data for Kraft paper come from ecoinvent.

Raw materials are transported to the manufacturing plant via diesel truck or rail. The following distances and modes of transport were modeled:

- MDI: 2414 km (1500 mi) by rail;

- Polyester polyol: 1384 km (860 mi) by rail (90 \%), 1384 km (860 mi) by truck (10 \%);

- Pentane: 2414 km (1500 mi) by truck;

- Remaining materials: 1609 km (1000 mi) by truck.

\section{Manufacturing}

According to Bayer MaterialScience (2011), polyiso plants consume primarily electricity and natural gas used to operate the laminator and associated operations support equipment, such as thermal oxidizers, storage areas, packaging machines, raw material pumps, offices, etc. A small amount of propane is used for fork lift trucks. Table 5-15 presents energy inputs and process outputs to produce 1 board-foot of foam, or $0.09 \mathrm{~m}^{2}\left(1 \mathrm{ft}^{2}\right)$ of $2.54 \mathrm{~cm}(1 \mathrm{in})$ thick polyiso foam. 
Table 5-15 Energy Inputs and Process Outputs for 1 Board-Foot Polyiso Foam

\begin{tabular}{|lcc|}
\hline Energy inputs & Unit & Quantity \\
\hline Electricity & MJ (kWh) & $0.0497(0.0138)$ \\
\hline Nat gas & MJ (Btu) & $0.0913(86.55)$ \\
\hline Propane & kg (lb) & $0.00015(0.00031)$ \\
\hline Outputs & Unit & Quantity \\
\hline Pentane to air & kg (lb) & $0.00013(0.00030)$ \\
\hline Waste scrap & board-foot & 0.01 \\
\hline
\end{tabular}

Based on review with polyiso plant manufacturers, approximately $2.5 \%$ of the pentane added to the foam is lost to air during manufacturing. Depending on the plant and local regulatory requirements, pentane is emitted directly to the atmosphere or to a thermal oxidizer for combustion. Only 13 plants out of 31 use thermal oxidizers to combust the pentane emissions. Since the majority of polyiso plants in North America do not use thermal oxidizers, the pentane is modeled as going directly to atmosphere (Bayer MaterialScience 2011).

Transportation and disposal of manufacturing waste scrap was modeled as going to an industrial landfill. It is assumed that a landfill for such non-hazardous waste is within $32 \mathrm{~km} \mathrm{(20} \mathrm{mi)} \mathrm{of} \mathrm{the}$ polyiso plant.

\subsection{Transportation to the Building Site through End-of-Life}

Transportation to the building site is modeled as $400 \mathrm{~km}$ (250 mi) by heavy-duty diesel truck (Bayer MaterialScience 2011).

Installation tape is used but is excluded since it is considered negligible. Scrap polyiso generated at installation is assumed to be $2 \%$ of the total, consistent with other foam products in this category. While the product may be recyclable, it is modeled as being sent to a landfill $32 \mathrm{~km}$ (20 $\mathrm{mi}$ ) from the building site. Data for the landfill come from waste management datasets in ecoinvent. Pentane release at installation is negligible.

Polyiso insulation has a functional lifetime of over 40 years so no replacement is needed during the 40-year study period. How insulation in the buildings affects operating energy during the prototype buildings' use phase is addressed in other sections of this report.

Polyiso insulation is modeled as disposed of in a landfill at end-of-life. End-of-life modeling includes transportation by heavy-duty diesel truck approximately $80 \mathrm{~km}$ (50 mi) to a C\&D landfill. Landfilled insulation is modeled based on ecoinvent end-of-life waste management process data. Per Bayer MaterialScience (2011), $50 \%$ of the total pentane in the product will have been released by end-of-life and $50 \%$ remains in the product.

\subsubsection{Windows}

BIRDS evaluates windows with a range of characteristics, including number of window panes, frames with and without thermal breaks, and glass films, fill, and coatings (argon gas fill, low-emissivity (low-E) coatings, tint, and reflective coatings). These windows are matched to the 
window performance characteristics (U-factor and solar heat gain coefficient (SHGC)) for each building type-location-standard edition combination.

Note that the window assumptions in the Reference Commercial Building Models are simplified for the estimates completed in BIRDS. The documentation for the simulation models includes a breakdown by operability as well as frame type, but there is no information provided on the combination of these two factors. Additionally, the window performance specifications are the same for all window glazing in a simulation. Therefore, BIRDS uses the fraction of windows that are operable and fixed combined with the single window type included in the RS Means baseline building cost estimate as shown in Table 5-16.

\section{Table 5-16 Baseline Window Types}

\begin{tabular}{|c|c|}
\hline Building Type & RS Means Baseline Window \\
\hline HOTEL06 & aluminum awning, insulated, 4-5 x 5-3 \\
\hline ELEMSC01 & $\begin{array}{l}\text { aluminum awning, insulated, } 4-5 \times 5-3 \\
\text { aluminum flush tube, thermo-break, } 2.25 \text { x 4.5, } 5 \times 6 \text { opening, } 2 \text { horizontals; glazing panel 1" } \\
\text { insulating tinted }\end{array}$ \\
\hline RETAIL01 & $\begin{array}{l}\text { Aluminum flush tube frame, for } 1 / 4 \text { "glass, } 1-3 / 4 " x \text { 4-1/2", 5'x6' opening, no intermediate } \\
\text { horizontals; Glazing panel, insulating, } 1 / 2 " \text { thick, } 2 \text { lites 1/8" float glass, clear }\end{array}$ \\
\hline FFRSTR01 & $\begin{array}{l}\text { Aluminum flush tube frame, for } 1 / 4 \text { "glass, } 1-3 / 4 " x \text { 4-1/2", 5'x6' opening, no intermediate } \\
\text { horizontals; Glazing panel, insulating, } 1 / 2 \text { " thick, } 2 \text { lites } 1 / 8 \text { " float glass, clear }\end{array}$ \\
\hline SDRSTR01 & $\begin{array}{l}\text { Aluminum flush tube frame, for 1/4"glass,1-3/4"x4", 5'x6' opening, no intermediate } \\
\text { horizontals; Glazing panel, insulating, } 1 / 2 " \text { thick, } 2 \text { lites 1/8" float glass, clear }\end{array}$ \\
\hline STRPMALL01 & $\begin{array}{l}\text { Aluminum flush tube frame, for 1/4"glass, } 1-3 / 4 " x 4 " \text { ", 5'x6' opening, no intermediate } \\
\text { horizontals; Glazing panel, insulating, } 1 / 2 \text { " thick, } 2 \text { lites } 1 / 8^{\prime \prime} \text { float glass, clear }\end{array}$ \\
\hline HIGHSC02 & $\begin{array}{l}\text { aluminum flush tube, thermo-break, } 2.25 \text { x 4.5, 5x6 opening, } 2 \text { horizontals; glazing panel 1/2" } \\
\text { insulating tinted }\end{array}$ \\
\hline OFFICE12 & $\begin{array}{l}\text { aluminum flush tube, thermo-break, } 2.25 \text { x 4.5, } 5 \times 6 \text { opening, no horizontals; glazing panel } \\
1 / 4 \text { " clear }\end{array}$ \\
\hline APART04 & Windows, aluminum, sliding, standard glass, 5' x 3' \\
\hline APART10 & Windows, aluminum, sliding, standard glass, 5' x 3' \\
\hline HEALTH03 & aluminum, picture unit, insulated, 3-4 x 5-0 \\
\hline OFFICE01 & Windows, aluminum, awning, insulated glass, 4'-5" x 5'-3" \\
\hline OFFICE03 & Windows, aluminum, awning, insulated glass, 4'-5" x 5'-3" \\
\hline HOTEL04 & Windows, aluminum, awning, insulated glass, 4'-5" x 5'-3" \\
\hline WAREHSE01 & Windows, aluminum, sliding, standard glass, 5' x 3' \\
\hline
\end{tabular}

The characteristics of these baseline windows are matched to the most comparable window type for which LCIA data is available, which are discussed in detail Section 5.5.3.1.1 through Section 5.5.3.1.4: aluminum operable sliding window, aluminum punched opening fixed window, aluminum fixed storefront window, and aluminum curtain wall window. For each of these windows, LCIA data has been developed for a range of window performance specifications based on the standard edition requirements given LCA and cost data restrictions. Each window U-factor-SHGC specification combination in the commercial building simulations is matched to the window characteristics (number of panes, frame type, operability, and other adjustments) required to meet the specifications based on Efficient Windows Collaborative 
(www.efficientwindows.org). Each simulation is matched to the window type and one of these potential set of window characteristics:

- $\quad$ Standard Glass Window

- Standard Glass Window with Tint

- Insulated Glass Window

- Insulated Glass Window with Tint

- Insulated Glass Window with Thermal Break Frame

- Insulated Glass Window with Thermal Break Frame and Tint

- Insulated Glass Window with Thermal Break Frame and Low-E Coating

- Insulated Glass Window with Thermal Break Frame and Tint and Low-E Coating

It is acknowledged that there are multiple window assembly options (combination of frame material, glass in-fill, and operability) for any building type in any climate zone; multiple window assembly options can be nearly identical in performance. The window assembly types presented in these tables are only one of many options available. As such, the window assembly combinations presented in this documentation are not endorsed or preferred over any other type of window assembly for the respective buildings in which they are used in BIRDS.

It should also be noted that window to wall ratios (WWRs) vary greatly for many different reasons. Those used in BIRDS are based solely on the DOE Reference Building Simulation Models, and may not be representative of WWRs for actual buildings of similar types.

BIRDS environmental performance data for the windows category was provided on a per-window basis for the operable sliding windows and fixed windows, and per $0.09 \mathrm{~m}^{2}\left(1 \mathrm{ft}^{2}\right)$ of typical or common size of fixed storefront and curtain wall windows. The flow diagram below presents the general system boundaries for the window category as it is modeled for BIRDS.

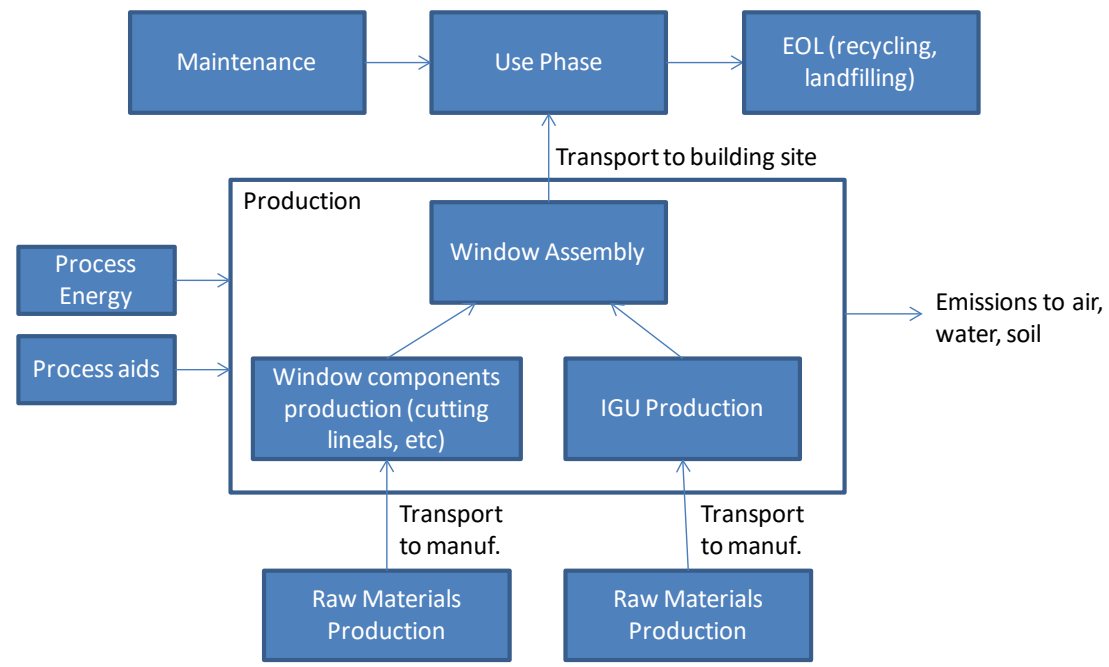

Figure 5-7 Windows System Boundaries

The large commercial window manufacturer provided electricity, natural gas energy, and net water use data based on one operable or fixed window, storefront window, and curtainwall 
window. For confidentiality purposes, these data are not shared in this documentation, however, they have been included in the models for all the window types.

\subsubsection{Window Upstream Materials Production through Manufacturing}

The LCIA assumptions and data for manufacturing of each of the four window types as well as the coatings that can be added to each window are discussed in detail below.

\subsection{Aluminum Operable Sliding Window Production}

Material take-off data for 2010 production were provided for an aluminum operable sliding window of dimensions $152.4 \mathrm{~cm}(5 \mathrm{ft})$ by $91.4 \mathrm{~cm}(3 \mathrm{ft})$. The following table provides general data on the dimensions and main components of a single- and double-pane window, and a double-pane window with a thermal break frame. A thermal break is a material that separates the interior and exterior of an aluminum (or other metal) frame. The low thermal conductivity of the material reduces heat transfer, making the metal window energy efficient. Due to the proprietary nature of the data, the full details of all the materials have been removed.

\section{Table 5-17 Characteristics and Components of the Aluminum Operable Window}

\begin{tabular}{|c|c|c|c|c|}
\hline \multirow[b]{2}{*}{ Description } & \multirow[b]{2}{*}{ Units } & \multicolumn{3}{|c|}{ Quantity } \\
\hline & & Single pane & Double pane & $\begin{array}{c}\text { Double pane w/ } \\
\text { thermo-br }\end{array}$ \\
\hline Weight of the finished sealed unit & kg (lb) & $33(72)$ & 47 (103) & $48(105)$ \\
\hline Dimension of the window & $\mathrm{m} 2(\mathrm{ft} 2)$ & $1.4(15)$ & $1.4(15)$ & $1.4(15)$ \\
\hline Depth of the finished sealed unit & cm (in) & $8.3(3.25)$ & $8.3(3.25)$ & $8.3(3.25)$ \\
\hline Extruded aluminum (frame) & kg (lb) & $16(35)$ & $15(33)$ & $15(33)$ \\
\hline IGU & kg (lb) & $15(32)$ & $29(65)$ & $29(65)$ \\
\hline Other components & kg (lb) & $2.10(4.64)$ & $2.11(4.65)$ & $2.95(6.50)$ \\
\hline
\end{tabular}

Greater than $99 \%$ of the mass of materials in the window were included and modeled for this window system. The aluminum frame is modeled as $50 \%$ primary and $50 \%$ secondary extruded aluminum, with the LCI data coming from the U.S. LCI Database. “Other components” include clips, weatherstrip, gaskets, hardware, sealant, and thermobreak materials. Clips, weatherstrip, and gaskets are modeled as PVC and rubber parts. Hardware is custom ordered so may vary with the window. For BIRDS, a mix of $50 \%$ stainless steel and $50 \%$ bronze has been modeled. The sealant is assumed to be silicone-based, and thermal break materials include glass-reinforced polyamide and PUR resin. U.S. LCI Database provided the production data for PVC while the ecoinvent database provided the data for the remaining materials.

Data for the insulated glass units (IGU) are compiled from two sources: the commercial window manufacturer and Salazar (2007, Table 5.4). These two data sources have been averaged into one "hypothetical” IGU with some categories having combinations of materials to increase representation of an IGU in the marketplace, which has an array of materials that can be used for various parts of the IGU. The bill of materials based on $0.09 \mathrm{~m}^{2}\left(1 \mathrm{ft}^{2}\right)$ of the IGU is as follows: 


\section{Table 5-18 Insulated Glass Unit Materials}

\begin{tabular}{|llrc|}
\hline IGU category & Material & $\mathbf{k g} / \mathbf{~ m}^{\mathbf{2}}$ & $\mathbf{l b} / \mathbf{f t}^{\mathbf{2}}$ \\
\hline Glass (2 panes) & Glass $(0.25)$ & 14.1 & 2.9 \\
\hline Gas filler & Argon & $2.01 \mathrm{E}-02$ & $4.12 \mathrm{E}-03$ \\
\hline Spacer & 50/50 Stainless St/Alum & $1.86 \mathrm{E}-01$ & $3.81 \mathrm{E}-02$ \\
\hline Primary sealant & Polyisobutylene & $7.70 \mathrm{E}-03$ & $1.58 \mathrm{E}-03$ \\
\hline Secondary sealant & 50/50 Polysulfide/PUR & $1.71 \mathrm{E}-01$ & $3.51 \mathrm{E}-02$ \\
\hline Desiccant & Silicone & $8.98 \mathrm{E}-02$ & $1.84 \mathrm{E}-02$ \\
\hline
\end{tabular}

The argon was calculated based on the volume between the panes of glass separated by $1.27 \mathrm{~cm}$ ( $0.5 \mathrm{in})$ and an argon density of $1.65 \mathrm{~kg} / \mathrm{m}^{3}\left(0.103 \mathrm{lb} / \mathrm{ft}^{3}\right)$. Salazar (2007) reported an escape of argon when the cavity is flushed prior to being sealed - approximately $95 \%$ more than the quantity in the finished unit. The manufacturer did not provide data on material losses. Since it is unknown if this quantity of escaped argon is high or low, a $50 \%$ escape is assumed for this model. The spacer, which separates the two panes of glass, can be made of an array of materials, including aluminum, stainless steel, and structural foam. Since no data were provided on an IGU with structural foam, a 50/50 assumption of aluminum and stainless steel was used. Salazar (2007) reported a loss of approximately $10 \%$ of the spacer; this has been factored in to the model. However, for both materials, the loss is recycled and not actually waste. The inner primary sealant, commonly polyisobutylene (PIB), is used to prevent leakage of the argon gas as well as penetration of water vapor into the space between the panes. An assumption of 50/50 polysulphide polymer and polyurethane as secondary sealants were used. The desiccant in the spacer, assumed to be a silicone product, is used to absorb moisture. Salazar (2007) reported a loss of approximately $0.7 \%$ and this has been factored into the model. Losses discussed in this paragraph are not included in the table above.

Note that for windows specified without argon filling, the IGU bill of materials is the same as the table above without the argon filling. Also, coatings and tints, when applicable, have been included in the model but are not included in the table.

The data for float glass come from ecoinvent, based on early 2000's European processes and technologies. Due to lack of available data on U.S. float glass production, the older European data were used. Processes in the data set include melting, cullet addition, forming (on a float bath), annealing by cooling in an oven (lehr), cutting of the glass, and storage. While this data set may not be representative of current U.S. production, it has been customized using U.S. energy and transportation data sets. Also, some transportation impacts have been removed, including transport between manufacturing plants and coating facilities, which per U.S. windows industry representatives exists for European operations but not for U.S. operations. As with other LCIA data, BIRDS will be updated to include improved data on glass production as resources (e.g. funding) permit.

The argon, stainless steel, polysulfide, and desiccant data come from ecoinvent. PIB come from ecoinvent; synthetic rubber is used as a general proxy for PIB. Aluminum and PUR data come from the U.S. LCI Database. 
No data were provided on the supplier distances to the manufacturer, so it is assumed that the materials to the manufacturing plant are transported an average of $600 \mathrm{~km}$ (373 mi) by heavy-duty diesel-fueled truck.

\subsection{Aluminum Punched Opening, Fixed Window Production}

Material take-off data for 2010 production were provided for an aluminum punched opening, fixed window of dimensions $135 \mathrm{~cm}$ (4 ft $5 \mathrm{in}$ ) by $160 \mathrm{~cm}$ (5 ft $3 \mathrm{in}$ ). Table 5-19 provides general data on the dimensions and main components of a single- and double-pane window, and a double-pane window with a thermal break frame. Due to the proprietary nature of the data, the full details of all of the materials have been removed.

\section{Table 5-19 Characteristics and Components of the Aluminum Fixed Window}

\begin{tabular}{|c|c|c|c|c|}
\hline \multirow[b]{2}{*}{ Description } & \multirow[b]{2}{*}{ Units } & \multicolumn{3}{|c|}{ Quantity } \\
\hline & & Single pane & Double pane & $\begin{array}{c}\text { Double pane w/ } \\
\text { thermo-br }\end{array}$ \\
\hline Weight of the finished sealed unit & kg (lb) & $39(85)$ & $66(145)$ & $67(148)$ \\
\hline Dimension of the window & $\mathrm{m}^{2}\left(\mathrm{ft}^{2}\right)$ & $1.4(15)$ & $1.4(15)$ & $1.4(15)$ \\
\hline Depth of the finished sealed unit & cm (in) & $5.1(2.0)$ & $5.1(2.0)$ & $5.1(2.0)$ \\
\hline Extruded aluminum (frame) & kg (lb) & $10(23)$ & $10(22)$ & $11(24)$ \\
\hline IGU & kg (lb) & $27(60)$ & $55(122)$ & $55(122)$ \\
\hline Other components & kg (lb) & $0.93(2.05)$ & $0.78(1.72)$ & $0.78(1.72)$ \\
\hline
\end{tabular}

Greater than $99 \%$ of the mass of materials in the window were included and modeled for this window system. The aluminum frame is modeled as 50/50 primary and secondary extruded aluminum, with the LCI data coming from the U.S. LCI Database. “Other components” include clips, gaskets, sealant, and thermal break materials. Clips and gaskets are modeled as PVC and rubber parts. The sealant is assumed to be silicone-based, and thermal break materials include glass-reinforced polyamide and PUR resin. U.S. LCI Database provided the production data for PVC while the ecoinvent database provided the data for the remaining materials. No data were provided on the supplier distances to the manufacturer, so it is assumed that the materials to the manufacturing plant are transported an average of $600 \mathrm{~km}$ (373 mi) by heavy-duty diesel-fueled truck.

The IGU for the fixed, punched opening window is the same as for the operable sliding window. Please refer to that section above.

\subsection{Aluminum Fixed Storefront Window Production}

Material take-off data for 2010 production were provided for an aluminum fixed storefront window of dimensions $203 \mathrm{~cm}$ (80 in) by $203 \mathrm{~cm}$ (80 in). Table 5-20provides general data on the dimensions and main components of a single- and double-pane window, and a double-pane window with a thermal break frame. Due to the proprietary nature of the data, the full details of all the materials have been removed. 
Table 5-20 Characteristics and Components of the Aluminum Storefront Window

\begin{tabular}{|lcccc|}
\hline Description & Units & $\begin{array}{c}\text { Single } \\
\text { pane }\end{array}$ & $\begin{array}{c}\text { Quantity } \\
\text { Double pane }\end{array}$ & $\begin{array}{c}\text { Double pane } \\
\text { w/thermal-br }\end{array}$ \\
\hline Weight of the finished sealed unit & $\mathrm{kg}(\mathrm{lb})$ & $78(173)$ & $141(310)$ & $133(293)$ \\
\hline Dimension of the window & $\mathrm{m}^{2}\left(\mathrm{ft}^{2}\right)$ & $4.1(44)$ & $4.1(44)$ & $4.1(44)$ \\
\hline Depth of the finished sealed unit & $\mathrm{cm}^{(\mathrm{in})}$ & $11.4(4.5)$ & $11.4(4.5)$ & $11.4(4.5)$ \\
\hline Extruded aluminum (frame) & $\mathrm{kg}(\mathrm{lb})$ & $24(52)$ & $30(66)$ & $21(46)$ \\
\hline IGU & $\mathrm{kg}(\mathrm{lb})$ & $54(118)$ & $109(240)$ & $109(240)$ \\
\hline Other components & $\mathrm{kg}(\mathrm{lb})$ & $1.8(4.0)$ & $1.9(4.3)$ & $3.2(7.2)$ \\
\hline
\end{tabular}

Greater than $99 \%$ of the mass of materials in the window were included and modeled for this window system. The aluminum frame is modeled as 50/50 primary and secondary extruded aluminum, with the LCI data coming from the U.S. LCI Database. "Other components" include clips, gaskets, sealant, and thermal break materials. Clips and gaskets are modeled as PVC and rubber parts. Thermal break materials include glass-reinforced polyamide and PUR resin. U.S. LCI Database provided the production data for PVC while the ecoinvent database provided the data for the remaining materials. No data were provided on the supplier distances to the manufacturer, so it is assumed that all materials to the manufacturing plant are transported 600 km (373 mi) by heavy-duty diesel-fueled truck.

The IGU for the fixed storefront window is the same as for the operable sliding window. Please refer to that section above.

\subsection{Aluminum Curtain Wall Window Production}

Material take-off data for 2010 production were provided for an aluminum curtain wall window of dimensions $203 \mathrm{~cm}$ (80 in) by $203 \mathrm{~cm}$ (80 in). Table 5-21 provides general data on the dimensions and main components of a single- and double-pane window, a double-pane window with a thermal break frame, and a triple-pane window with a thermal break frame. Due to the proprietary nature of the data, the full details of all the materials have been removed.

Table 5-21 Characteristics and Components of the Aluminum Storefront Window

\begin{tabular}{|lccccc|}
\hline Description & Units & Single pane & $\begin{array}{c}\text { Double } \\
\text { pane }\end{array}$ & $\begin{array}{c}\text { Quantity } \\
\text { Double pane } \\
\text { w/ thermal-br }\end{array}$ & $\begin{array}{c}\text { Triple pane w/ } \\
\text { thermal-br }\end{array}$ \\
\hline Weight of the finished sealed unit & $\mathrm{kg}(\mathrm{lb})$ & $105(232)$ & $157(346)$ & $158(348)$ & $213(469)$ \\
\hline Dimension of the window & $\mathrm{m}^{2}\left(\mathrm{ft}{ }^{2}\right)$ & $4.1(44)$ & $4.1(44)$ & $4.1(44)$ & $4.1(44)$ \\
\hline Depth of the finished sealed unit & $\mathrm{cm}(\mathrm{in})$ & $17.8(7.0)$ & $17.8(7.0)$ & $17.8(7.0)$ & $17.8(7.0)$ \\
\hline Extruded aluminum (frame) & $\mathrm{kg}(\mathrm{lb})$ & $49(108)$ & $47(103)$ & $49(107)$ & $51(112)$ \\
\hline IGU & $\mathrm{kg}(\mathrm{lb})$ & $53(116)$ & $107(236)$ & $107(236)$ & $159(350)$ \\
\hline Other components & $\mathrm{kg}(\mathrm{lb})$ & $3.4(7.5)$ & $3.5(7.8)$ & $2.3(5.1)$ & $3.1(6.7)$ \\
\hline
\end{tabular}

Greater than $99 \%$ of the mass of materials in the window were included and modeled for this window system. The aluminum is modeled as primary extruded aluminum, with the LCI data coming from the U.S. LCI Database. “Other components” include clips, gaskets, sealant, and 
thermal break materials. Clips and gaskets are modeled as PVC and rubber parts. Thermal break materials include glass-reinforced polyamide and PUR resin. U.S. LCI Database provided the production data for PVC while the ecoinvent database provided the data for the remaining materials. No data were provided on the supplier distances to the manufacturer, so it is assumed that all materials to the manufacturing plant are transported $600 \mathrm{~km}$ (373 mi) by heavy-duty diesel-fueled truck.

The IGU for the curtain wall is essentially the same as for the operable sliding window. Please refer to that section above.

\subsection{Coatings}

Low-emissivity (low-E) coatings, reflective coatings, and tinted windows have been included in the windows modeling to meet different performance characteristics of the windows. Coatings are used to improve the insulation properties of the glass by reflecting visible light and infrared radiation. Low-E coating is modeled using the coating details of ecoinvent's "flat glass, coated" data set as a starting point. The technology used at this plant is based on a cathodic sputtering technology which involves depositing thin silver and other layer(s) on the glass. Per the ecoinvent documentation, the raw materials used for sputtering are bismuth, silver and nickelchrome. The quantity of $1.19 \mathrm{E}-4 \mathrm{~kg}$ (2.62 E-4 lb) metals per kg was divided into three to account for $1 / 3$ nickel, $1 / 3$ chromium, and $1 / 3$ silver. It is acknowledged that these data are approximate.

Per the Windows for High Performance Commercial Buildings website, reflective coatings usually consist of thin metallic or metal oxide layers and come in various metallic colors including silver, gold, and bronze. ${ }^{11}$ Due to lack of available other data, the reflective coating data use the same quantity of coating as low-E but apply silver, gold and bronze data sets to account for a range of reflective coatings.

Tint is obtained by adding small amounts of metal oxides during glass manufacturing, coloring the glass uniformly. For BIRDS, iron oxide has been assumed to be the mineral additive for the tint, and it is modeled as applied at an assumed rate of $0.1 \%$ of the weight of glass.

\subsubsection{Window Transportation, Use, Maintenance, and End-of-Life}

Transportation of the window to the building site is modeled using an assumed average of 805 km (500 mi) by heavy-duty (combination) diesel fuel-powered truck.

Installing windows is primarily a manual process; no energy or emissions are included in the model for this. Windows come to the building fully assembled and custom-ordered to fit so there is generally no installation waste.

The commercial windows are modeled as having a functional lifetime of over 40 years so no replacement is needed during the 40 -year study period. Maintenance in the model includes

\footnotetext{
${ }^{11}$ Site developed jointly by the University of Minnesota and Lawrence Berkeley National Laboratory. Found at: http://www.commercialwindows.org/reflective.php.
} 
weatherstripping and sealing (discussed below). All operational energy-related aspects of the window are addressed in other sections of this report.

Weatherstrip is modeled as a thermoplastic elastomer. Data for the thermoplastic, as ethylene propylene diene monomer (EPDM) rubber, come from ecoinvent (as synthetic rubber). For BIRDS, an EPDM weatherstrip has been modeled in the amount of $0.0064 \mathrm{~kg}$ per $0.3 \mathrm{~m}$ (0.014 lb per $\mathrm{ft})$. Weatherstrip is assumed to perform at its optimal level an average of 7.5 years (Vigener, 2012) so is modeled as replaced every 7.5 years.

Different perimeter sealants can be used for different applications. For BIRDS, a silicone sealant has been modeled in the amount of $0.018 \mathrm{~kg}$ per $0.3 \mathrm{~m}(0.04 \mathrm{lb}$ per $\mathrm{ft})$. This is based on the average amount of sealant needed for perimeter depth/width gaps of $0.64 \mathrm{~cm} / 0.64 \mathrm{~cm}$ $(0.25 \mathrm{in} / 0.25 \mathrm{in})$ and $0.64 \mathrm{~cm} / 1.27 \mathrm{~cm}(0.25 \mathrm{in} / 0.5 \mathrm{in}) .{ }^{12}$ Data for the sealant comes from ecoinvent, primarily its silicone product. The sealant is modeled as being replaced every 15 years (Vigener, 2012). Other maintenance, such as glass and/or window frame cleaning, frame repainting or recoating, hardware adjustment or replacement, etc., are not included in the analysis.

The aluminum frame portion of the window is modeled as recycled at end of life, and the 0-100 recycling methodology has been applied. For this, system expansion is applied; the production of the same amount of virgin aluminum that is in the frame is subtracted out of the system, crediting the system with an avoided burden based on the reduced requirement for virgin material production in the next life cycle. Likewise, recycled content in the aluminum adds some of the burden to the product system to share the burden with the previous life cycle. ${ }^{13}$

The remaining parts of the window, including the IGU, are disposed of in a landfill. Fiberglass and composite frames are modeled as disposed of in a landfill. End-of-life modeling includes transportation by diesel-fuel powered truck approximately $80 \mathrm{~km}$ (50 mi) to a C\&D landfill or to recycling. The portions of the window going to landfill are modeled based on ecoinvent end of life waste management process data.

\subsubsection{HVAC}

The HVAC systems for each building type in BIRDS were based on the system defined in the Commercial Reference Building Models as discussed in Chapter 3 and shown in Table 3-6. In several building types there are multiple systems installed and operated. These systems are mapped to the closest match in the available LCIA data as shown in Table 5-6.

\footnotetext{
${ }^{12}$ Amount calculated on an on-line sealant usage calculator, found at http://www.tremcosealants.com/technicalresources/calculators/sealant-calculator.aspx.

${ }^{13}$ For more information on the approach to modeling metals at end of life, see Atherton (2006) and World Steel Association (2011).
} 
Table 5-6 HVAC Equipment Mapping to LCIA Data

\begin{tabular}{|ll|}
\hline \multicolumn{1}{|c|}{ Model HVAC Type } & \multicolumn{1}{c|}{ Heating LCIA Mapping } \\
\hline Packaged VAV AC Unit & Packaged Unit - Rooftop, Multizone -15 tons \\
\hline Packaged Terminal AC (PTAC) & Packaged Unit - Rooftop, Single Zone -29 tons \\
\hline Packaged AC Unit (PACU) & Packaged Unit - Rooftop, Single Zone -29 tons \\
\hline $\begin{array}{l}\text { Packaged AC Unit - Split } \\
\text { System }\end{array}$ & Other Packaged Units - Air Cooled Condensing Unit - Split System - 76 tons \\
\hline Centrifugal chiller & Packaged Water-Cooled Chiller (includes cooling tower) - 260 tons \\
\hline Air-Cooled Chiller & Packaged Air-Cooled Chiller (includes fan coil) - 46.66 tons \\
\hline Electric cabinet heater & Electric Furnace - 34.12 MBH \\
\hline Unit Heater & Electric Furnace - 34.12 MBH \\
\hline Gas Furnace & Gas Furnace - 34.12 MBH \\
\hline Gas Boiler & Gas-Fired Boiler - 163 MBH \\
\hline Water Source Heat Pump & Air-to-Air Heat Pump - 3-ton \\
\hline Air-to-Air Heat Pump & Air-to-Air Heat Pump - 3-ton \\
\hline
\end{tabular}

BIRDS environmental performance data for the HVAC equipment evaluated was provided on a per-unit basis. The flow diagram below presents the general system boundaries for the HVAC equipment as it is modeled for BIRDS. The subsections below describe the life cycle modeling of the equipment. The HVAC system size varies across building energy standard editions because changing the thermal characteristics of the building envelope alters the heating and cooling loads of the building. The EnergyPlus whole-building energy simulations "autosize" the HVAC system to determine the system size. The LCIA data for each of the HVAC equipment types is normalized on a per unit of capacity and multiplied by the HVAC capacity for a given building simulation to calculate the LCIA estimates for the HVAC system.

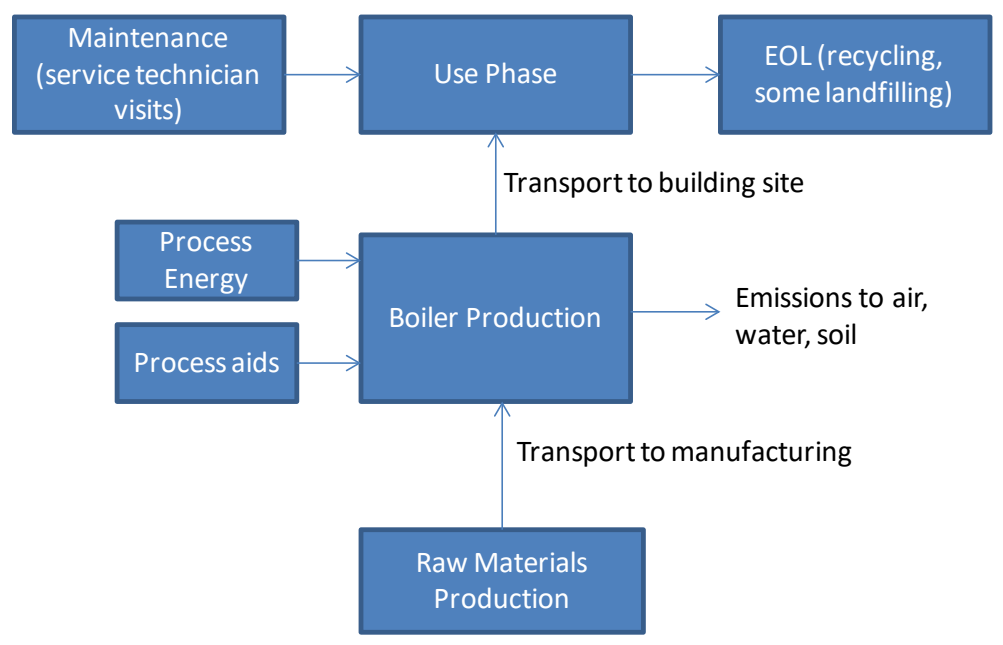

Figure 5-8 HVAC System Boundaries - Boiler Example

\subsubsection{Boilers}

Boilers provide the steam or hot water to various parts of the buildings. The following types of boilers were evaluated (Table 5-22): 
Table 5-22 Boilers Included in BIRDS

\begin{tabular}{|c|c|c|}
\hline Type of Boiler, construction & $\begin{array}{l}\text { Gross output in kW } \\
\left(\mathrm{MBH}^{14}\right)\end{array}$ & Notes \\
\hline Cast iron, natural gas- or propane-fired & Steam, $70(240)$ & Includes standard controls and insulated jacket \\
\hline Cast iron, natural gas- or propane-fired & Steam, $82(280)$ & Includes standard controls and insulated jacket \\
\hline $\begin{array}{l}\text { Cast iron, natural gas- or propane- } \\
\text { fired }\end{array}$ & Steam, 48 (163) & $\begin{array}{l}\text { Includes standard controls and insulated } \\
\text { jacket }\end{array}$ \\
\hline Cast iron, natural gas- or propane-fired & Steam, 448 (1530) & Includes standard controls and insulated jacket \\
\hline Cast iron, natural gas- or propane-fired & Steam, 1958 (6680) & Includes standard controls and insulated jacket \\
\hline Cast iron, gas- and oil-fired & Hot water, 1758 (6000) & \\
\hline
\end{tabular}

\subsection{Upstream Materials Production through Manufacturing}

Data were compiled from publicly-available manufacturer-specific literature data (Table 5-23). These specific models were chosen since their literature provided adequate data to compile a rough estimate of the material quantities.

\section{Table 5-23 Boiler Models}

\begin{tabular}{|ll|l|}
$\begin{array}{l}\text { Output, kW } \\
\text { (MBH) }\end{array}$ & Manufacturer \& model & \multicolumn{1}{l|}{ Data Source for dimensions, weight* } \\
\hline $\begin{array}{l}\text { Steam, 70 (240) } \\
\text { Steam, 82 (280) } \\
\text { Steam, 48 (163) }\end{array}$ & $\begin{array}{l}\text { Weil McClain, EGH 65-75 } \\
\text { Weil McClain, EGH 75-85 }\end{array}$ & $\begin{array}{l}\text { Weil McClain EG-PEG-EGH } \\
\text { Gas Fired Boilers Series 4 - Boiler Manual, } \\
\text { Part No. 550-110-639/0706, Table 15 \& p. 34 diagrams. }\end{array}$ \\
\hline Steam, 448 (1 530) & Weil McClain, EGH 50-55 & $\begin{array}{l}\text { Weil McClain LGB } \\
\text { Gas Fired Boiler - Boiler Manual, } \\
\text { Part Number 550-141-186/0703, Sections 12, 14. }\end{array}$ \\
\hline $\begin{array}{l}\text { Steam, 1 958 (6 680) } \\
\text { Water, 1 758 (6 000) }\end{array}$ & $\begin{array}{l}\text { Weil McClain, (94)H2494 } \\
\text { Weil McClain, (94)H2194-22944 }\end{array}$ & $\begin{array}{l}\text { Weil-McLain 94 } \\
\text { Water and steam boilers - for Gas, Light Oil, \& Gas/Light } \\
\text { Oil-Fired Burners - Boiler Manual, } \\
\text { Part No. 550-110-275/0810, p.42 \& Fig 5. }\end{array}$ \\
\hline \multirow{2}{*}{ *Weil-McLain boiler manuals retrieved at: http://www.weil-mclain.com/en/our-products/boilers/commercial-boilers.aspx } \\
\hline
\end{tabular}

As a starting point for the bill of materials, data on the cast iron content of a $938 \mathrm{~kW}$ (3 200 $\mathrm{MBH}$ ) Veissmann water boiler which has a cast iron heat exchanger was assumed (Veissmann Vitorond, July 2010), ${ }^{15}$ and this content was approximately $96 \%$. The assumption was then made that this percentage was the same for other boilers with cast iron heat exchangers, and a representative from Weil McClain confirmed that it is "roughly $95 \%$ \%. ${ }^{16}$ Thus, cast iron was assumed for $94 \%$ for smaller output boilers and $96 \%$ for larger ones. The remaining $4 \%$ to $6 \%$ of the total weights for the steel jacket and volume of insulation were calculated based on dimensions of the units and an insulation thickness of $1.3 \mathrm{~cm}(0.5 \mathrm{in})$ of fiberglass batt (thickness confirmed by Weil McClain). The weight of fiberglass was calculated using a density of $22.6 \mathrm{~kg} / \mathrm{m}^{3}\left(1.41 \mathrm{lb} / \mathrm{ft}^{3}\right)$ (see insulation section). The remaining weight was assumed to be cold

\footnotetext{
${ }^{14} \mathrm{MBH}=1000 \mathrm{Btu} / \mathrm{hr}$.

${ }^{15}$ For example: the Viessmann model Vitorond VD2 1080.

${ }^{16}$ Correspondence with Weil McClain, February 2012.
} 
rolled steel and a small amount of copper in the controls. Table 5-24 provides the bill of materials for each boiler based on the above assumptions and boiler weight data from literature.

Table 5-24 Boiler Bill of Materials

\begin{tabular}{|lccccc|}
\hline Output, kW (MBH) & Total kg (lb) & $\begin{array}{c}\text { Cast Iron } \\
\mathbf{k g}(\mathbf{l b})\end{array}$ & $\begin{array}{c}\text { Steel } \\
\mathbf{k g}(\mathbf{l b})\end{array}$ & $\begin{array}{c}\text { Insulation } \\
\mathbf{k g}(\mathbf{l b})\end{array}$ & $\begin{array}{c}\text { Copper } \\
\mathbf{k g}(\mathbf{l b})\end{array}$ \\
\hline Steam, 70 (240) & $320(705)$ & $301(663)$ & $18(40)$ & $1.0(2.3)$ & $0.11(0.25)$ \\
\hline Steam, 82 (280) & $358(789)$ & $337(742)$ & $20(45)$ & $1.1(2.5)$ & $0.11(0.25)$ \\
\hline Steam, 48 (163) & $\mathbf{2 6 5 ( 5 8 5 )}$ & $\mathbf{2 4 9 ( 5 5 0 )}$ & $\mathbf{1 5}(\mathbf{3 3})$ & $\mathbf{0 . 9}(\mathbf{2 . 0})$ & $\mathbf{0 . 1 1 ( 0 . 2 5 )}$ \\
\hline Steam, 448(1 530) & $2064(4551)$ & $1981(4368)$ & $79(175)$ & $3.1(6.9)$ & $0.36(0.8)$ \\
\hline Steam, 1 958 (6 680) & $10161(22400)$ & $9754(21503)$ & $397(876)$ & $9.1(20.0)$ & $0.45(1.0)$ \\
\hline Water, 1 758 (6 000) & $9219(20324)$ & $8850(19510)$ & $361(795)$ & $8.4(18.5)$ & $0.45(1.0)$ \\
\hline
\end{tabular}

Ecoinvent data were used for the cast iron. Data for the cold rolled steel come from World Steel Association (2011), and data for copper come from ICA (2012). The fiberglass insulation was modeled as described in the insulation section.

Raw materials are modeled as transported to the manufacturing plant via diesel truck an assumed average distance of $805 \mathrm{~km}$ (500 mi).

No manufacturing data were available for commercial boilers, so as proxy, an ecoinvent data set for a $100 \mathrm{~kW}$ (341 MBH) oil boiler was used (ecoinvent Report No. 5, 2007). The ecoinvent data summary describes data for the production energy to be estimated and coming from a 1998 environmental report (Table 5-25). In general, the data being estimated and of older vintage are not necessarily representative. More representative data will be incorporated when it becomes available.

\section{Table 5-25 Boiler Manufacturing Data}

\begin{tabular}{|lc|}
\hline & Quantity for 580 kg boiler \\
Energy source & $\mathbf{1 0 0 ~ k W}$ \\
\hline Electricity, medium voltage MJ (Btu) & $1195(1132831)$ \\
\hline Natural gas in industrial furnace MJ (Btu) & $1920(1819809)$ \\
\hline Light fuel oil in industrial furnace MJ (Btu) & $1010(957295)$ \\
\hline Tap water liter (gal) & $741(196)$ \\
\hline
\end{tabular}

Since manufacturing larger or smaller units requires more or less energy, respectively, these data were normalized up or down based on the total weights of the units.

\subsection{Transportation to the Building Site through End-of-Life}

Transportation of the equipment to the building site is modeled to be an assumed average of 644 km (400 mi) by heavy-duty diesel fuel-powered truck.

It is assumed that a qualified service technician comes to the building site to check and/or service the unit one time per year to ensure optimal performance and lifetime. It is assumed that the qualified technician is within a $24 \mathrm{~km}$ (15 mi) service radius. The $24 \mathrm{~km}$ (15 mi), driven in a gasoline-powered van, is allocated amongst other service visits for that technician, assuming that 
the same technician is making more than one service call during that trip. Assuming the technician makes 5 service calls in one day, one-fifth of the impacts from driving $24 \mathrm{~km} \mathrm{(15} \mathrm{mi)}$ are allocated to the product, or $4.8 \mathrm{~km}$ (3 mi). Data for a van come from ecoinvent. Unplanned service visits (i.e., unanticipated issues that require a service technician) are not included in the modeling assuming that the building personnel follow the maintenance and care correctly.

A lifetime of 35 years has been assumed, based on the statement in a boiler brochure: "It's not uncommon for Weil-McLain cast iron boilers to last 35 years or more." ${ }^{17}$ Shah (2008) backs this number up as well.

At the end of life, it is assumed that the packaged unit is removed from the building and its metal parts are recycled, especially since the vast majority is recoverable metal, fully recyclable, and the equipment is easy to recover and remove from the building. See Footnote 13 for the recycling methodology used. The fiberglass is modeled as landfilled, and a distance of $48 \mathrm{~km}$ (30 mi) to the landfill in a heavy-duty diesel truck has been modeled. The landfill is modeled based on ecoinvent end of life waste management process data.

\subsubsection{Package Units}

Packaged units, or rooftop units, are air handlers designed to be installed for use on rooftops. An air handler is an appliance used to condition and circulate air through a building as part of the building's HVAC system. A split system is an air conditioner split by the condensing unit located on the outside of the building and the evaporator in a furnace or air handler inside the building. Four packaged units and one split system condensing unit were evaluated:

\section{Table 5-26 Packaged Units Characteristics in BIRDS}

\begin{tabular}{|ll|}
\hline Type of HVAC System & Designated Tonnage \\
\hline Rooftop air conditioner - single zone & $\mathbf{2 9 . 1 7}$ \\
\hline Rooftop air conditioner - multizone & $\mathbf{1 5 . 0 0}$ \\
\hline Rooftop air conditioner - multizone & 58.33 \\
\hline Rooftop air conditioner - multizone & 79.16 \\
\hline Air cooled condensing unit - split system & 76.66 \\
\hline
\end{tabular}

The commercial HVAC equipment manufacturer provided electricity for assembly (punching, shearing, forming parts), lights, and fans, natural gas usage, and propane for forklifts. Data were provided on a full-facility basis for 2010 and calculated on the basis of one unit, based on total production time for the various lines of HVAC equipment produced at the facility. For confidentiality purposes, these manufacturing data are not shared in this documentation, however, they have been included in the model, i.e., normalized for each size of unit in this category.

${ }^{17}$ Boiler lifetime from Weil-McLain LGB brochure - C-805 (1111). 


\subsection{Rooftop Air Conditioner Upstream Materials Production through Manufacturing}

Data for a commercial sized rooftop air conditioner were provided by a global producer of commercial HVAC equipment. The full bill of materials for the system was provided for BIRDS. Table 5-27 provides general data on the main components of the equipment but due to the proprietary nature of the data, the full details of all of the materials and the weight of the components have been removed.

\section{Table 5-27 Packaged Units Main Components}

\begin{tabular}{|lll|}
\hline Component name & Description \& Material & Notes \\
\hline Housing & Painted galvanized steel of varying thicknesses & \\
\hline Condenser Coils & Aluminum & \\
\hline Supply Fan & Mostly steel \& a 10 HP motor & Assume 20\% motor, $80 \%$ steel \\
\hline Evaporator Coil & Copper tubing, Aluminum fin stock & \\
\hline Condenser Fans & Includes 1-hp motors, fan blades, basket. & Assume $80 \%$ motors, 20\% steel \\
\hline Economizer Damper & Aluminum & \\
\hline Control Panel & Sheet metal housing, electrical components & \\
\hline Compressor & Steel, motors, copper & \\
\hline Refrigerant & R410A & Assume $90 \%$ steel, $10 \%$ rubber \\
\hline Misc parts & Nuts, bolts, gaskets & \\
\hline
\end{tabular}

This product requires a roof curb, which is a steel structure that supports HVAC equipment on top of a building. The curb is included in the model and is assumed to be galvanized steel.

Data for galvanized steel sheet for the housing come from World Steel Association (2011). The total weight for the compressor was given without a breakdown of the individual components. Data for a compressor was therefore modeled based on the percentages of the materials in a compressor reported in Biswas (2011):

\section{Table 5-28 Compressor Bill of Materials}

\begin{tabular}{|lcc|}
\hline Material & Weight kg (lb) & $\mathbf{\%}$ \\
\hline Steel & $15.5(34)$ & 9 \\
\hline Copper & $7.5(17)$ & 4 \\
\hline Aluminum & $3.0(6.6)$ & 2 \\
\hline Cast iron & $141(311)$ & 84 \\
\hline Total & $\mathbf{1 6 7}(\mathbf{3 6 8})$ & $\mathbf{1 0 0}$ \\
\hline
\end{tabular}

Aluminum for the condenser coils, evaporator coil, and economizer damper is modeled as a 50/50 mix of primary and secondary extruded aluminum which come from the U.S. LCI Database.

For the motors, a data set for an electric car motor, from ecoinvent, has been used as a guideline for the general material make-up for an electric motor. This data set includes the general materials in the motor, including rolled steel (75\% of total), aluminum (approximately $16 \%$ ), and copper wire (9\%). U.S. LCI data sets were used for the aluminum, World Steel Association (2011) for the steel, and ICA (2012) for the copper. 
Steel in the supply fan, compressor, and miscellaneous parts comes from cold rolled steel from World Steel Association (2011) and data for copper come from ICA (2012).

Control panel: The World Steel Association (2011) data for cold rolled steel was used for the sheet metal housing component of the control panel, which made up $80 \%$. Not enough data were available on the contents of this remaining part to model the electrical components. Synthetic rubber data come from ecoinvent.

R-410a data are based on a 50/50 share of difluoromethane (R-32) and pentafluoroethane (R125). Due to lack of available production data on both of these chemicals, proxies were used. Trifluoromethane (HFC-23) was used as a proxy for difluoromethane and 1,1,difluoroethane (HFC 152a) was used as proxy for R125. While proxies were used for the production aspect of the chemicals, any release of these was based on the release of R-32 and R-125, not the proxy chemicals, so that ozone depletion impact remains zero and global warming potential impact is calculated appropriate to R-410A.

Raw materials are modeled as transported to the manufacturing plant via diesel truck an assumed average distance of $805 \mathrm{~km}$ (500 mi).

\subsection{Other Package Units Upstream Materials Production through Manufacturing}

For lack of available data for the other products in this category, this bill of materials was normalized for each size of packaged unit included in BIRDS. The exception to this was the quantity of refrigerant used for each and the unit weights, both of which could be obtained or extrapolated for different models of rooftop air conditioners. Table 5-29 summarizes the data sources and details of the rooftop air conditioner units to meet the NIST specs: 
Table 5-29 Packaged Unit Models

\begin{tabular}{|c|c|c|c|c|}
\hline HVAC System & Product this is based on ${ }^{18}$ & $\begin{array}{l}\text { Weight } \\
\text { kg (lb) }\end{array}$ & $\begin{array}{l}\text { R-410a } \\
\text { charge } \\
\text { kg (lb) }\end{array}$ & $\begin{array}{l}\text { Curb } \\
\text { weight } \\
\text { kg (lb) }\end{array}$ \\
\hline $\begin{array}{l}\text { Rooftop air conditioner - single } \\
\text { zone }\left(929 \mathrm{~m}^{2}\left(10000 \mathrm{ft}^{2}\right)\right. \\
29.17 \text { ton })\end{array}$ & $\begin{array}{l}\text { Maverick II commercial } \\
\text { packaged rooftop system, } 30 \\
\text { ton } 19\end{array}$ & $\begin{array}{c}1637 \\
(3610)\end{array}$ & $11.3(25)$ & $192(423)$ \\
\hline $\begin{array}{l}\text { Rooftop air conditioner - } \\
\text { multizone }\left(279 \mathrm{~m}^{2}\left(3000 \mathrm{ft}^{2}\right), 15.00\right. \\
\text { ton) }\end{array}$ & $\begin{array}{l}\text { Voyager Packaged Rooftop Air } \\
\text { Conditioners, } 15 \text { ton }^{20}\end{array}$ & $\begin{array}{c}1035 \\
(2281)\end{array}$ & $5.2(11.4)$ & $192(423)$ \\
\hline $\begin{array}{l}\text { Rooftop air conditioner - multizone } \\
\left(2323 \mathrm{~m}^{2}\left(25000 \mathrm{ft}^{2}\right), 58.33 \text { ton }\right)\end{array}$ & $\begin{array}{l}\text { IntelliPak Packaged Rooftop Air } \\
\text { Conditioners, } 60 \text { ton }^{21}\end{array}$ & $\begin{array}{c}3782 \\
(8338)\end{array}$ & $20.1(44.4)$ & $234(515)$ \\
\hline $\begin{array}{l}\text { Rooftop air conditioner - multizone } \\
\left(2323 \mathrm{~m}^{2}\left(25000 \mathrm{ft}^{2}\right), 79.16 \text { ton }\right)\end{array}$ & $\begin{array}{l}\text { IntelliPak Packaged Rooftop Air } \\
\text { Conditioners, } 75 \text { ton }^{22}\end{array}$ & $\begin{array}{c}4011 \\
(8843)\end{array}$ & $25.2(55.6)$ & $277(610)$ \\
\hline
\end{tabular}

\subsection{Air Cooled Condensing Unit - Split System Upstream Materials Production through Manufacturing}

Bill of materials for the split system air cooled condensing unit were based on data for a Carrier Corporation air conditioner from Shah (2008). The mass of the Carrier Corporation 24ACR3 Comfort 13 Series (1-1/2 to 5 nominal tons) condenser unit in the article was normalized up to the mass of a commercial split system air-cooled condensing unit available on the market. The Trane split system condensing unit with remote evaporator chiller was chosen based on available data on weight and refrigerant quantity provided in company literature.

Legutko (2000, p.3) describes an evaporator as "a direct-expansion, finned, tubular coil that has refrigerant inside the tubes. A fan draws air across the finned exterior of the tubes and delivers it to the spaces being conditioned. Standard coil construction consists of copper tubes with aluminum fins mounted in a galvanized steel frame.” The remote evaporator's bill of materials was based on Legutko (2000); the dimensions of the Trane remote evaporator and a gauge chart were used to estimate the galvanized steel frame, and assumptions were made on the remaining components. ${ }^{23}$ Table 5-30 presents the bill of materials for the condenser unit and remote evaporator.

\footnotetext{
${ }^{18}$ The packaged units in the table representing the NIST systems were chosen based on available data on weight and/or refrigerant quantity provided in company literature. The products listed do not imply endorsement or product preference or quality.

${ }^{19}$ Daikin McQuay Catalog 250-6: Maverick II commercial packaged rooftop systems: Heating \& Cooling Models MPS015F - 075E, base weight from Table 36, curb weight from Table 37. Refrigerant data extrapolated based on other sources.

${ }^{20}$ Trane Product Catalog: Packaged Rooftop Air Conditioners: Voyager ${ }^{\mathrm{TM}}$ Cooling and Gas/Electric - 121/2-25 Tons, 60 Hz, RT-PRC028-EN, Nov. 2011. Weight Table 88, refrigerant Table 1.

${ }^{21}$ Trane Product Catalog, Packaged Rooftop Air Conditioners IntelliPak ${ }^{\mathrm{TM}}$ - S*HL, S*HK - 20 - 130Tons -AirCooled Condensers - $60 \mathrm{~Hz}$, RT-PRC036-EN, April 2012, weights from Table 84. Refrigerant is extrapolated based on other Trane systems.

${ }^{22}$ Trane Product Catalog, Packaged Rooftop Air Conditioners IntelliPak ${ }^{\mathrm{TM}}$ - S*HL, S*HK - 20 - 130Tons —AirCooled Condensers - $60 \mathrm{~Hz}$, RT-PRC036-EN, April 2012, weights from Table 84. Refrigerant is extrapolated based on other Trane systems.

${ }^{23}$ Trane Product Catalog: Split System Condensing Units, Fig. 41 (assumed 14 gauge), Tables 1, 11, 12.
} 
Table 5-30 Split System Bill of Materials

\begin{tabular}{|c|c|c|c|}
\hline Unit & Material & $\begin{array}{l}\text { Quantity in Residential } \\
\text { System kg (lb) }\end{array}$ & $\begin{array}{l}\text { Quantity in 80-ton } \\
\text { Compressor kg (lb) }\end{array}$ \\
\hline Condenser unit & $\begin{array}{l}\text { Steel } \\
\text { Galvanized steel } \\
\text { Copper } \\
\text { Aluminum } \\
\text { Total }\end{array}$ & $\begin{array}{c}78(172) \\
35(77) \\
17(37) \\
17(37) \\
\mathbf{1 4 7}(\mathbf{3 2 4}) \\
\end{array}$ & $\begin{array}{c}1189(2621) \\
534(1176) \\
259(571) \\
259(571) \\
2241(\mathbf{4 9 4 0 )}\end{array}$ \\
\hline & $\begin{array}{l}\text { Refrigerant R-22 } \\
\text { Refrigerant R-410a }\end{array}$ & $\begin{array}{c}6(13) \\
\text { n/a }\end{array}$ & $\begin{array}{c}\mathrm{n} / \mathrm{a} \\
26(57)\end{array}$ \\
\hline Unit & Material & $\begin{array}{l}\text { Quantity in Residential } \\
\text { System kg (lb) }\end{array}$ & $\begin{array}{l}\text { Quantity in Evaporator } \\
\text { kg (lb) }\end{array}$ \\
\hline Remote Evaporator & $\begin{array}{l}\text { Galvanized steel } \\
\text { Copper } \\
\text { Aluminum } \\
\text { Other steel parts } \\
\text { Total }\end{array}$ & $\begin{array}{l}\mathrm{n} / \mathrm{a} \\
\mathrm{n} / \mathrm{a} \\
\mathrm{n} / \mathrm{a} \\
\mathrm{n} / \mathrm{a} \\
\mathrm{n} / \mathrm{a}\end{array}$ & $\begin{array}{c}20(45) \\
54(120) \\
6(13) \\
14(30) \\
94(208)\end{array}$ \\
\hline
\end{tabular}

The material data for elements in the systems are described above.

\subsection{Packaged Units Transportation to the Building Site through End-of-Life}

Transportation of the equipment to the building site is modeled to be an assumed average of 644 $\mathrm{km}$ (400 mi) by heavy-duty diesel fuel-powered truck.

It is assumed that a qualified service technician comes to the building site to check and service the unit every three years to ensure optimal performance and lifetime. It is assumed that the qualified technician is within a $24 \mathrm{~km}(15 \mathrm{mi})$ service radius. The $24 \mathrm{~km}(15 \mathrm{mi})$, driven in a gasoline-powered van, is shared amongst other service visits for that technician, assuming that the same technician is making more than one service call during that trip. Assuming the technician makes 5 service calls in one day, one-fifth of the impacts from driving $24 \mathrm{~km}$ (15 mi) are allocated to the product, or $4.8 \mathrm{~km}$ (3 mi). Data for a van come from ecoinvent. Unplanned service visits (i.e., unanticipated issues that require a service technician) are not included in the modeling assuming that the building personnel follow the maintenance and care correctly.

For the packaged units, a lifetime of 15 years has been assumed, based on an average of a 10 to 20-year life span for a non-coastal application. ${ }^{24}$ For the split system unit, a lifetime of 20 years for the condenser unit and 25 years for the air handler have been assumed, based on the lifetimes reported by Shah (2008).

For this analysis, $0.5 \%$ per year of the total refrigerant for each unit is assumed to leak.

At the end of life, it is assumed that the packaged unit is removed from the building and recycled, especially since most of the parts are valuable recoverable metal, fully recyclable, and relatively easy to recover and remove from the building. The recycling methodology used is described in Footnote 13. The curb is not removed, as a new unit is installed in its place. The

${ }^{24}$ Based on discussions with a manufacturer of packaged units. 
non-metal components are modeled as landfilled. A distance of $48 \mathrm{~km}$ (30 mi) to the landfill in a heavy-duty diesel truck has been modeled. The landfill is based on ecoinvent waste management process data.

\subsubsection{Packaged Chillers}

Air-cooled packaged chillers with scroll compressors and water-cooled packaged chillers with screw compressors were modeled for BIRDS. Additionally, these chillers were modeled with a fan coil unit, and the water-cooled chillers included a cooling tower. These were evaluated for the following applications:

\section{Table 5-31 Packaged Chillers Characteristics in BIRDS}

\begin{tabular}{|lc|}
\hline Type of HVAC System & $\begin{array}{c}\text { Designated } \\
\text { Tonnage }\end{array}$ \\
\hline Air-cooled packaged chiller with scroll compressor & $\mathbf{4 6 . 6 6}$ \\
\hline Air-cooled packaged chiller with scroll compressor & 93.33 \\
\hline Water-cooled packaged chiller with screw compressor & 140.00 \\
\hline Water-cooled packaged chiller with screw compressor & 190.00 \\
\hline Water-cooled packaged chiller with screw compressor & $\mathbf{2 3 0 . 0 0}$ \\
\hline
\end{tabular}

One manufacturer of water-cooled packaged chillers provided electricity and other energy data on a full-facility basis for 2010. From those data, the energy data were calculated based on one unit, based on total production time for the various lines of HVAC equipment produced at the facility. For confidentiality purposes, these manufacturing data are not shared in this documentation, however, they have been included in the model.

\subsection{Air-Cooled Chillers - Upstream Materials Production}

No data were available for specifically the air-cooled packaged chiller, so the bill of materials used for the rooftop air conditioner in the previous section was used as a proxy (see Table 5-27). The data were customized to these packaged chillers in terms of the unit weights and quantity of refrigerant used, both of which could be obtained for models of air cooled packaged chillers that would meet the NIST specifications. Table 5-32 summarizes the data sources and details of the air-cooled packaged chillers to meet the NIST specs:

\section{Table 5-32 Air Scroll Packaged Chiller Model}

\begin{tabular}{|c|c|c|c|}
\hline HVAC System & Product this is based on & $\begin{array}{l}\text { Weight } \\
\text { kg (lb) }\end{array}$ & $\begin{array}{l}\text { R-410a charge } \\
\text { kg (lb) }\end{array}$ \\
\hline $\begin{array}{l}\text { Air scroll packaged chiller with fan coil } \\
\text { unit, } 1858 \mathrm{~m}^{2}\left(20000 \mathrm{ft}^{2}\right), 46.66 \text { ton }\end{array}$ & McQuay AGZ 045D (45 ton) & $1551(3420)$ & $40(88)$ \\
\hline $\begin{array}{l}\text { Air scroll packaged chiller with fan coil unit, } \\
3716 \mathrm{~m}^{2}\left(40000 \mathrm{ft}^{2}\right), 93.33 \text { ton }\end{array}$ & McQuay AGZ 090 (90 ton) & 2539 (5605) & 78 (172) \\
\hline
\end{tabular}


The data and assumptions used for the air scroll chillers, including production of raw materials and transportation of materials to the manufacturing facility, are found in the section above on rooftop air conditioners.

\subsection{Water-Cooled Chillers - Upstream Materials Production}

No data on water-cooled screw compressor chillers with a range of tonnage of 140 to 230 were available, so the bill of materials for a 2500-ton water-cooled centrifugal chiller from Institute for Construction and Environment (2011) was used as a proxy. It is acknowledged that water screw compressors and centrifugal chillers are different technologies but these data were used for lack of other data available and because they perform the function of cooling.

Data in the EPD were collected in 2010; the EPD provided the major assemblies of the unit in terms of weights of each, shown in Table 5-33. Even though major assemblies were identified, the EPD provided the material composition in terms of percentage of the total mass, not in terms of materials within each assembly (next table). Thus, it was impossible to switch out assemblies to be able to better customize the data for the water screw chillers.

Table 5-33 Centrifugal Chiller Major Assemblies

\begin{tabular}{|lrlc|}
\hline Major Assemblies & kg & (lb) & \% of total \\
\hline Compressor & 13226 & $(29157)$ & 36 \\
\hline Compressor Install & 2300 & $(5070)$ & 6 \\
\hline Condenser & 11460 & $(25265)$ & 31 \\
\hline Controls & 14 & $(31)$ & 0.003 \\
\hline Economizer & 303 & $(668)$ & 1 \\
\hline Evaporator Tubes & 7463 & $(16453)$ & 20 \\
\hline Motors & 165 & $(363)$ & 0.4 \\
\hline Oil Tank & 232 & $(511)$ & 1 \\
\hline Purge & 585 & $(1290)$ & 2 \\
\hline Shell & 581 & $(1281)$ & 2 \\
\hline Unit Assembly & 417 & $(919)$ & 1 \\
\hline TOTAL & $\mathbf{3 6 7 4 5}$ & $(\mathbf{8 1} \mathbf{0 0 8})$ & $\mathbf{1 0 0}$ \\
\hline
\end{tabular}

Table 5-34 Centrifugal Chiller Materials

\begin{tabular}{|lrlc|}
\hline Material / subcomponent & $\mathbf{k g}$ & $\mathbf{( l b )}$ & \% of total \\
\hline Steel & 16536 & 36454 & 45 \\
\hline Copper & 10656 & 23492 & 29 \\
\hline cast iron & 8084 & 17822 & 22 \\
\hline Aluminum & 735 & 1620 & 2 \\
\hline Motor & 735 & 1620 & 2 \\
\hline TOTAL & $\mathbf{3 6 7 4 5}$ & $\mathbf{8 1 ~ 0 0 8}$ & $\mathbf{1 0 0}$ \\
\hline
\end{tabular}

Note: brass was listed but as $0 \%$ so is not included in the table. 
The material quantities were normalized to the weights of different models of water screw packaged chillers, and were customized in terms of the quantity of refrigerant used for each. Table 5-35 summarizes the data sources and details of the water-cooled packaged chillers to meet the NIST specs:

Table 5-35 Water-cooled Packaged Chiller Models

\begin{tabular}{|llcc|}
\hline HVAC System & Product this is based on & \multicolumn{1}{|l|}{$\begin{array}{l}\text { Weight } \\
\text { kg (lb) }\end{array}$} & $\begin{array}{c}\text { R-134a Charge } \\
\text { (Both Circuits) }\end{array}$ \\
\hline Water- cooled packaged chiller, 140.00 ton & $\begin{array}{l}\text { Trane RTWD 60 Hz standard } \\
\text { efficiency (140 Ton) }\end{array}$ & 3016 (6 650) & $118(260)$ \\
\hline Water- cooled packaged chiller, 190.00 ton & $\begin{array}{l}\text { Trane RTWD 60 Hz high } \\
\text { efficiency (200 Ton) }\end{array}$ & $3705(8$ 168) & $124(273)$ \\
\hline $\begin{array}{l}\text { Water- cooled packaged chiller, 230.00 } \\
\text { ton }\end{array}$ & $\begin{array}{l}\text { Trane RTWD 60 Hz high } \\
\text { efficiency (220 Ton) }\end{array}$ & $\mathbf{4 0 7 9 ~ ( 8 ~ 9 9 3 )}$ & $\mathbf{1 6 5 ~ ( 3 6 4 )}$ \\
\hline * Trane Catalog Series R Helical Rotary Liquid Chillers, Tables 1, 67. & & \\
\hline
\end{tabular}

Data on cold rolled steel come from World Steel Association (2011) and data on copper come from ICA (2012). Ecoinvent (2018) was used for the cast iron. Aluminum is modeled as a mix of primary and secondary extruded aluminum which comes from the U.S. LCI Database.

For the motors, a data set for an electric car motor, from ecoinvent, has been used as a guideline for the general material make-up for an electric motor. This data set includes the general materials in the motor, including rolled steel (75\% of total), aluminum (approximately $16 \%$ ), and copper wire (9\%). U.S. LCI data sets were used for the aluminum, World Steel Association (2011) for the steel, and ICA (2012) for the copper.

Data for 134a come from Ecoinvent (2018). Raw materials are modeled as transported to the manufacturing plant via diesel truck an assumed average distance of $805 \mathrm{~km}(500 \mathrm{mi})$.

\subsection{Transportation to the Building Site through End-of-Life}

Transportation of the equipment to the building site is modeled based on an assumed average of $644 \mathrm{~km}$ (400 mi) by heavy-duty diesel fuel-powered truck.

It is assumed that a qualified service technician comes to the building site to check and service the unit one time per year to ensure optimal performance and lifetime. It is assumed that the qualified technician is within a $24 \mathrm{~km}(15 \mathrm{mi})$ service radius. The $24 \mathrm{~km}(15 \mathrm{mi})$, driven in a gasoline-powered van, is shared amongst other service visits for that technician, assuming that the same technician is making more than one service call during that trip. Assuming the technician makes 5 service calls in one day, one-fifth of the impacts from driving $24 \mathrm{~km}$ (15 mi) are allocated to the product, or $4.8 \mathrm{~km}$ (3 mi). Data for a van come from ecoinvent. Unplanned service visits (i.e., unanticipated issues that require a service technician) are not included in the modeling assuming that the building personnel follow the maintenance and care correctly.

Bakane (2009) estimates a lifetime of 10 years for the scroll chiller and 17.5 years for the screw compressor (based on an average of 15 yrs to 20 yrs). For this analysis, 0.5 percent per year of the total refrigerant in each is assumed to leak, and this is modeled as recharged by the service technician. 
At the end of life, it is assumed that these chillers are removed from the building and recycled, especially since most of the parts are valuable recoverable metal, fully recyclable, and relatively easy to recover and remove from the building. The recycling methodology used is described in Footnote 13. The non-metal components are modeled as landfilled; landfill data come from ecoinvent. A distance of $48 \mathrm{~km}$ (30 mi) to the landfill in a heavy-duty diesel truck has been modeled.

\subsection{Fan Coils}

A fan coil is a device that has a heating or cooling coil and a fan, and is used to provide heat or air conditioning to the space in which it is installed. The bill of materials for the fan coil comes from Shah (2008, Table 3), as follows:

Table 5-36 Fan Coil Bill of Materials

\begin{tabular}{|lc|}
\hline Material & $\begin{array}{c}\text { Weight } \\
\text { kg (lb) }\end{array}$ \\
\hline Steel & $48(106)$ \\
\hline Galvanized steel & $26(57)$ \\
\hline Copper & $2(4)$ \\
\hline Total & $\mathbf{7 6 ( 1 6 8 )}$ \\
\hline
\end{tabular}

Data for both cold rolled steel and galvanized steel come World Steel Association (2011) and data for copper come from ICA (2012). The fan coil is modeled as having a lifetime of 25 years, consistent with Shah (2008). At the end of life, it is assumed that the fan coil is recycled; the recycling methodology used is described in Footnote 13.

\subsection{Cooling Towers}

One cooling tower has been modeled to be used in conjunction with the water-cooled chillers in BIRDS. Per a Baltimore Aircoil Company (BAC) sales representative, the BAC Series 1500 cooling tower is the most appropriate for a commercial application needing a nominal tonnage in the range of 190 to 200 which generally represents an average for the water-cooled chillers in BIRDS). The bill of materials was compiled for the BAC 15201 (a single cell unit) from BAC Series 1500 Cooling Tower Specification (2010), which provided the following major components and subcomponents/parts. 
Table 5-37 Cooling Tower Components and Parts

\begin{tabular}{|c|c|}
\hline Component & Subcomponents / Parts \\
\hline Cooling Tower & Panels, Panel edges, Panel finish \\
\hline Cold Water Basin & $\begin{array}{l}\text { Panels, Drain/Clean out connection, Make-up valve, Float shell, Float fill, Float } \\
\text { connection }\end{array}$ \\
\hline Water Outlet & Water outlet connection, Lift out strainers, Anti-vortexing device \\
\hline Water Distribution System & $\begin{array}{l}\text { Water inlet, Integral strainer, Open gravity type basin, Gravity flow nozzle, } \\
\text { Basin weir, Metering orifice, Lift-off distribution covers }\end{array}$ \\
\hline Fans & Fan blades, Fan cylinder, Fan guard \\
\hline Bearings & Bearings, Grease, Seals \\
\hline Fan Drive & Powerband \\
\hline Sheaves & Sheaves \\
\hline Fan Motor & Motor, Enclosure, Finish on winding, shafts and bearings \\
\hline Fill and Drift Eliminators & Fill \& Integral drift eliminators \\
\hline Air Inlet Louvers & Air inlet louvers \\
\hline $\begin{array}{l}\text { Basin Water Level Sensing } \\
\text { and Control }\end{array}$ & $\begin{array}{l}\text { Enclosure, controls, water level, standpipe Venting, Standpipe mounting } \\
\text { hardware }\end{array}$ \\
\hline Accessories & $\begin{array}{l}\text { Basin heaters, vibration cutout switch, basin sweeper piping, air intake option, } \\
\text { platform, ladder, louver face platform, internal platform }\end{array}$ \\
\hline Equipment Controls & Enclosed controls \\
\hline Mounting Support & Support Structure \\
\hline
\end{tabular}

Any available data on these items, including specified surface areas, volumes, and weights were recorded. Some data on materials were provided by the literature while materials for other subcomponents and parts were assumed. The masses of the parts and materials were calculated using material densities and the manufacturer's published data. Table 5-38 presents the compiled bill of materials totaling the published mass of the BAC 15201 (1 $942 \mathrm{~kg}$ (4 $280 \mathrm{lb}))^{25}$

${ }^{25}$ BAC Product and Application Handbook -Series 1500 Engineering Data on Cooling Tower (no date), p. B58. 
Table 5-38 Cooling Tower Bill of Materials

\begin{tabular}{|lcc|}
\hline \multicolumn{1}{|c}{$\begin{array}{c}\text { Material / component } \\
\text { description }\end{array}$} & \multicolumn{2}{c|}{ Mass } \\
\cline { 2 - 3 } kg & (lb) \\
\hline Galvanized steel sheet & 1060 & $(2337)$ \\
\hline cold rolled steel & 146 & $(321)$ \\
\hline Aluminum & 38 & $(84)$ \\
\hline Cast iron & 2.3 & $(5.0)$ \\
\hline Equipment control assemblies & 6.4 & $(14)$ \\
\hline electric heater & 6.8 & $(15)$ \\
\hline Epoxy polyester & 3.7 & $(8.2)$ \\
\hline HDPE & 1.8 & $(4.0)$ \\
\hline Heavy-gauge steel & 161 & $(354)$ \\
\hline Polypropylene & 1.8 & $(4.0)$ \\
\hline Polystyrene & 0.1 & $(0.2)$ \\
\hline PVC & 379 & $(835)$ \\
\hline Stainless Steel & 8.7 & $(19)$ \\
\hline Fiberglass reinforced polyester & 109 & $(239)$ \\
\hline Motor & 18 & $(40)$ \\
\hline Total & $\mathbf{1 9 4 2}$ & $\mathbf{( 4 2 8 0 )}$ \\
\hline
\end{tabular}

Data for the galvanized steel sheet, cold rolled steel, and heavy-gauge steel come from World Steel (2011). U.S. LCI Database provided data for HDPE, PVC, general purpose polystyrene, polypropylene, and aluminum (assumed 50/50 primary and secondary).

Ecoinvent provided data on the cast iron, control assemblies, stainless steel, epoxy resin (for the epoxy polyester), and fiberglass reinforced polyester. For the motor, a data set for an electric car motor, from ecoinvent, has been used as a guideline for the general material make-up of an electric motor. This data set includes the general materials in the motor, including rolled steel (75\% of total), aluminum (approximately $16 \%$ ), and copper wire (9\%).

At the end of life, it is assumed that the cooling tower is removed from the building and recycled, especially since most of the materials are valuable recoverable metal and relatively easy to recover and remove from the building. The recycling methodology used is described in Footnote 13.

\subsubsection{Natural Gas and Electric Furnaces}

Natural gas and electric furnaces are typically installed in residential and smaller commercial buildings. This section will describe the data and approach implemented in estimating the LCIAs for these heating equipment types.

\subsection{Upstream Materials Production through Manufacturing}

LCA practitioners often seek bill of materials data when conducting life-cycle assessments. However, there was no bill of materials data available for furnaces installed in residential and 
small commercial settings, so proxy data were used. Athena Sustainable Materials Institute (2003) provided teardown data for a medium efficiency Lennox sealed combustion furnace, manufactured in 1985. These data were used with adjustments. The mass of the Lennox, $91 \mathrm{~kg}$ (200 lb), was normalized to the mass of an efficient natural gas furnace currently available on the market that weighs approximately $56 \mathrm{~kg}(124 \mathrm{lb})^{26,27}$. These data were supplemented by Yang, Zmeureanu et al. (2008), which provided data for a blower motor, replacing that of the older Lennox, to account for the more efficient furnace. Table 5-39 provides the materials in the natural gas furnace; the notes column identifies the adjustments made.

\section{Table 5-39 Natural Gas Furnace Bill of Materials}

\begin{tabular}{|lccl|}
\hline \multicolumn{2}{c}{ Mass } \\
\hline Material & kg & lb & Notes \\
\hline Aluminum & 1.02 & 2.25 & $\begin{array}{l}\text { Yang (2008) air blower data, replacing the Al in } \\
\text { Athena (2003) }\end{array}$ \\
\hline Brass & 0.05 & 0.11 & \\
\hline Ceramic & 0.04 & 0.08 & \\
\hline Circuit board, transistors & 0.05 & 0.11 & \\
Copper & 2.20 & 4.85 & $\begin{array}{l}\text { Yang (2008) air blower data, replacing the Cu } \\
\text { in Athena (2003) }\end{array}$ \\
\hline $\begin{array}{l}\text { Fiberglass insulation (foil- } \\
\text { lined) }\end{array}$ & 0.27 & 0.60 & \\
\hline Galvanized Steel & 21.86 & 48.19 & Steel paired down to meet the Rheem weight \\
\hline PET & 0.38 & 0.83 & \\
\hline PVC & 0.45 & 0.99 & \\
\hline Powder coating & 0.19 & 0.42 & \\
\hline Rubber & 0.02 & 0.04 & \\
\hline Steel & 29.79 & 65.66 & $\begin{array}{l}\text { Yang (2008) air blower and Athena (2003). } \\
\text { Steel paired down to meet the Rheem weight }\end{array}$ \\
\hline Total & $\mathbf{5 6 . 3}$ & $\mathbf{1 2 4 . 1}$ & \\
\hline
\end{tabular}

For the electric furnace, the mass of the Lennox was normalized down to the mass of the electric furnace in BIRDS, a unit currently on the market. ${ }^{28}$ Data for the blower motor were provided by Yang (2008). Table 5-40 presents the bill of materials with data adjustments.

\footnotetext{
${ }^{26}$ Weight of the Standard Rheem Classic 95 \% efficiency natural gas furnace (RGRC-07-RBGS) from Rheem Classic Series, Upflow Gas Furnaces, Physical Data and Specifications, Form No. G11-527, p.4.

${ }^{27}$ This source states a total shipping weight of $62.1 \mathrm{~kg}(137 \mathrm{lb})$. The mass of the equipment itself was assumed to be $91 \%$ of the total weight, based on other Rheem product published weights relative to their shipping materials.

${ }^{28}$ Rheem Air Handlers, Form No. H11-524 REV. 8, Unit Dimensions \& Weights, p.4, model 4221/4821.
} 


\section{Table 5-40 Electric Furnace Bill of Materials}

\begin{tabular}{|lccl|}
\hline & \multicolumn{3}{c}{ Mass } \\
Material & $\mathbf{k g}$ & $\mathbf{l b}$ & Notes \\
\hline Aluminum & 1.02 & 2.25 & Yang (2008), replacing the Al in Athena (2003) \\
\hline Brass & 0.05 & 0.11 & \\
\hline Ceramic & 0.04 & 0.08 & \\
\hline Circuit board, transistors & 0.05 & 0.11 & \\
\hline Copper & 2.20 & 4.85 & Yang (2008), replacing the Cu in Athena (2003) \\
\hline Fiberglass insulation (foil- lined) & 0.27 & 0.60 & \\
\hline Galvanized Steel & 34.04 & 75.04 & Steel paired down to meet the Rheem weight \\
\hline PET & 0.38 & 0.83 & \\
\hline PVC & 0.45 & 0.99 & \\
\hline Powder coating & 0.32 & 0.71 & Model assumes 0.095 kg powder per $\mathrm{m}^{2}$ (from \\
\hline Rubber & 0.02 & 0.04 & \\
\hline Steel & 29.17 & 64.31 & $\begin{array}{l}\text { Yang (2008) air blower and Athena (2003). Steel paired } \\
\text { down to meet the Rheem weight }\end{array}$ \\
\hline Total & $\mathbf{6 8 . 0 0}$ & $\mathbf{1 4 9 . 9}$ & \\
\hline
\end{tabular}

The steel is assumed to be cold-rolled; this and the galvanized steel come from World Steel Association (2011). Aluminum is modeled as a 50/50 mix of primary and secondary extruded aluminum using data from NREL (2015)the U.S. LCI Database. Data for copper come from International Copper Association (ICA) (2012). Data for PET and PVC come from the US LCI database. Ecoinvent provided the data for brass, ceramics, integrated circuit boards, rubber (as synthetic rubber), and powder coating. The fiberglass insulation was modeled as described in the insulation section of this report.

Raw materials are modeled as transported to the manufacturing plant via diesel truck an assumed average distance of $805 \mathrm{~km}$ (500 mi).

Because no manufacturing data were available for residential/small commercial furnaces, an ecoinvent data set for a $10 \mathrm{~kW}$ (34 MBH) oil boiler was used as a proxy (Ecoinvent 2007). The ecoinvent dataset description states that the $10 \mathrm{~kW}$ low- $\mathrm{NO}_{\mathrm{x}}$ or condensing boiler data may be applied for residential/small commercial furnaces (Ecoinvent 2007). The production energy is stated to be estimated from a 1998 environmental report. Even though this proxy is considered not to be representative of current practice; however its use is considered sufficient for comparison purposes.

Table 5-41 shows data for a boiler of approximately $150 \mathrm{~kg}$ (331 lb). Since manufacturing the smaller units is assumed to require less energy, these data were normalized based on the total weights of the natural gas and electric furnaces. 
Table 5-41 Furnace Manufacturing

\begin{tabular}{|lc|}
\hline Energy source & Quantity for 10 kW boiler \\
\hline Electricity, medium voltage, MJ (Btu) & $294(81.7)$ \\
\hline Natural gas in industrial furnace, MJ (Btu) & $424(401874)$ \\
\hline Light fuel oil in industrial furnace, MJ (Btu) & $236(223685)$ \\
\hline Tap water, liter (gal) & $182(48)$ \\
\hline
\end{tabular}

\subsection{Transportation to the Building Site through End-of-Life}

Transportation of the equipment to the building site is modeled as an assumed average of $644 \mathrm{~km}$ (400 mi) by heavy-duty diesel fuel-powered truck.

It is assumed that a qualified service technician comes to the building site to check and/or service the unit one time every three years to ensure optimal performance and lifetime. It is assumed that the qualified technician is within a $24 \mathrm{~km}$ (15 mi) service radius. This distance, driven in a gasoline-powered van, is allocated amongst other service visits, assuming that the same technician is making more than one service call during that trip. Assuming the technician makes 5 service calls in one day, one-fifth of the impacts from driving $24 \mathrm{~km} \mathrm{(15} \mathrm{mi)} \mathrm{are} \mathrm{allocated} \mathrm{to}$ the product, or $4.8 \mathrm{~km}$ (3 mi). Environmental flow data associated with the operation of the van comes from ecoinvent. Unplanned service visits (i.e., unanticipated issues that require a service technician) are not included in the model, assuming that the homeowner adequately follows the maintenance and care guidelines.

A lifetime of 16 years has been assumed for both gas and electric furnaces, based on the National Association of Home Builders (NAHB) Research Center (2007), a study on life expectancy of home components. ${ }^{29}$

At the end-of-life, it is assumed that the furnaces are sent for recycling to recover valuable metal using the "0-100 recycling methodology" discussed previously in Section 5.5.3. What cannot be recovered, i.e., any non-metallic parts, are modeled as landfilled, assuming a distance of $48 \mathrm{~km}$ (30 $\mathrm{mi}$ ) to the land fill with transport via heavy-duty diesel truck. The landfill is modeled based on ecoinvent end-of-life waste management process data.

\subsubsection{Residential/Small Commercial Air Conditioners}

Residential/small commercial air conditioners for BIRDS are modeled as split systems with outdoor and indoor components that provide a condenser and evaporative heat exchanger, respectively. BIRDS includes LCA data for 3-ton residential air conditioners with SEERs of 13, 14, 18, and 21. SEER is defined by the Air Conditioning, Heating, and Refrigeration Institute (AHRI) as total heat removed from the conditioned space during the annual cooling season, expressed in Btu's, divided by the total electrical energy consumed by the air conditioner or heat pump during the same season, expressed in watt-hours (AHRI 2008). Eighteen SEER is considered to be a high rating relative to other models; based on informal discussions with industry members, only a small percentage of the market is currently at 18 SEER. An even

\footnotetext{
${ }^{29}$ NAHB (2007), Table 1, Section 15 gives furnaces a life expectancy range of 15 to 20 years.
} 
smaller percentage is beyond 18 SEER, so 21 SEER is considered to be exceptionally high, providing an extremely efficient system.

\subsection{Upstream Materials Production through Manufacturing}

Bill of materials for the outside unit (condenser) shown in Table 5-42 came from De Kleine (2009). The condensing unit is based on a tear-down of a 3-ton, 10 SEER unit manufactured in approximately 2001. The components' materials were assumed by Four Elements, except where noted.

\section{Table 5-42 Condenser Unit Bill of Materials}

\begin{tabular}{|c|c|c|c|c|}
\hline \multicolumn{5}{|c|}{ Mass } \\
\hline Main Component & Material Breakdown & kg & Lb & Notes \\
\hline \multirow[t]{4}{*}{ Compressor } & Aluminum & 0.53 & 1.16 & \multirow{4}{*}{$\begin{array}{l}\text { Compressor breakdown from (Biswas and } \\
\text { Rosano 2011), Fig } 1 .\end{array}$} \\
\hline & Cast iron & 24.74 & 54.54 & \\
\hline & Copper & 1.32 & 2.90 & \\
\hline & Steel & 2.72 & 6.00 & \\
\hline \multirow[t]{2}{*}{ Coil Assembly } & Copper (tubing) & 3.95 & 8.71 & \multirow{2}{*}{$\begin{array}{l}\text { Approx. } 50 \% \text { copper tubing, } 50 \% \text { aluminum } \\
\text { fins. (De Kleine, 2009) }\end{array}$} \\
\hline & Aluminum (fins) & 3.95 & 8.71 & \\
\hline \multirow[t]{3}{*}{ Fan Motor } & Copper wire & 1.08 & 2.37 & \multirow{3}{*}{$\begin{array}{l}\text { Assumed to have a composition of } 25 \% \text { copper } \\
\text { wire, } 72 \% \text { steel, and } 3 \% \text { polyamide } \\
\text { (De Kleine 2009) }\end{array}$} \\
\hline & Steel & 3.10 & 6.83 & \\
\hline & Polyamide & 0.13 & 0.28 & \\
\hline Unit Wall & Galvanized steel & 3.10 & 6.83 & \\
\hline Base & Galvanized steel & 2.70 & 5.95 & \\
\hline Top Cover & Galvanized steel & 1.90 & 4.19 & \\
\hline Fan Guard & Stainless steel & 1.60 & 3.53 & \\
\hline \multirow{2}{*}{$\begin{array}{l}\text { Refrigerant Line } \\
\text { service valve }\end{array}$} & Brass (service valves) & 0.75 & 1.65 & \\
\hline & Rubber & 0.25 & 0.55 & \\
\hline Wire Guard & Stainless steel & 0.80 & 1.76 & \\
\hline Fan Blade & Steel & 0.60 & 1.32 & \\
\hline Misc Fasteners & Steel & 0.30 & 0.66 & \\
\hline Capacitor & Sheet metal (steel) & 0.30 & 0.66 & \\
\hline \multirow[t]{2}{*}{ Relay Switch } & Copper wiring & 0.10 & 0.22 & \multirow{2}{*}{$\begin{array}{l}\text { Assumed to be } 50 \% \text { copper wiring and } 50 \% \\
\text { nylon polymer (De Kleine 2009) }\end{array}$} \\
\hline & Nylon polymer & 0.10 & 0.22 & \\
\hline Copper Wiring & Copper wire & 0.20 & 0.44 & \\
\hline Total & & 54.20 & 119.49 & \\
\hline
\end{tabular}

De Kleine's teardown was for a SEER 10 unit, but the bill of materials for the higher SEERs were required for his study. To do this, De Kleine created a weight function using survey data and manufacturer specification sheets on condenser units from several different brands ranging in efficiency from 10 SEER to 18 SEER and ranging in capacity from 1.5 tons to 5 tons of cooling. This weight function was used to calculate the mass of each of the condenser units in BIRDS as follows, enabling the adjustment of the bill of materials to the different masses as shown in Table 5-43. See De Kleine (2009) for more detail. 
Table 5-43 Condenser Unit Masses

\begin{tabular}{|lccccc|}
\hline & SEER 10 & SEER 13 & SEER 14 & SEER 18 & SEER 21 \\
\hline Factor & 1 & 1.36 & 1.49 & 1.98 & 2.34 \\
\hline Mass in kg (lb) & $54.2(119.5)$ & $74.1(163.5)$ & $80.8(178.1)$ & $107.4(236.7)$ & $127.3(280.7)$ \\
\hline
\end{tabular}

These values corresponded with De Kleine (2009), and are consistent with manufacturers' products of the same SEER ratings.

The evaporator coil indoor unit was modeled as being housed in the electric furnace. De Kleine (2009) approximated the inside coil assembly for a 13 SEER system to weigh $15.9 \mathrm{~kg}$ (35 lb) and be composed of $50 \%$ copper tubing and $50 \%$ aluminum fins. The refrigerant line, connecting the outdoor and indoor units, was modeled as 20 feet of copper tubing weighing $4.5 \mathrm{~kg}(9.9 \mathrm{lb})$ (De Kleine 2009). The air conditioning system uses R-410a refrigerant, and data for the quantity of refrigerant used in each of the SEERs studied (shown in Table 5-44) was calculated using refrigerant mass function developed by De Kleine (2009).

\section{Table 5-44 Refrigerant Quantities}

\begin{tabular}{|lcccc|}
\hline & SEER 13 & SEER 14 & SEER 18 & SEER 21 \\
\hline R-410a in kg (lb) & $3.3(7.3)$ & $3.6(7.9)$ & $5.0(10.9)$ & $6.5(14.4)$ \\
\hline
\end{tabular}

The steel data, assumed to be cold-rolled, and the galvanized steel data come from the World Steel Association (2011). Aluminum is modeled as a 50/50 mix of primary and secondary extruded aluminum using data from the U.S. LCI Database. Data for copper tube, sheet, and wire come from International Copper Association (ICA) (2012). Ecoinvent provided the data for the cast iron, stainless steel, brass, rubber (as synthetic rubber), and polyamide.

R-410a data are based on a 50/50 share of difluoromethane (R-32) and pentafluoroethane (R125). Due to lack of available production data on both chemicals, proxies were implemented. Trifluoromethane (HFC-23) was used as a proxy for difluoromethane and 1,1,difluoroethane (HFC-152a) was used as proxy for R-125. Note: while proxies were used for the production aspect of the chemicals, any release of these was based on the release of R-32 and R-125, not the proxy chemicals, so that ozone depletion impact remains zero and global warming potential impact is calculated appropriate to R-410a.

Raw materials are modeled as transported to the manufacturing plant via diesel truck an assumed average distance of $805 \mathrm{~km}$ (500 mi).

No manufacturing data were available for residential/small commercial air conditioners, so as proxy, an ecoinvent data set for a $10 \mathrm{~kW}$ (34 MBH) oil boiler was used. Since manufacturing the smaller units is assumed to require less energy and resources than the $150 \mathrm{~kg}(331 \mathrm{lb})$ boiler, these data were normalized based on the total weights of the air conditioners. 


\subsection{Transportation to the Building Site through End-of-Life}

Transportation of the equipment to the building site is modeled assuming a heavy-duty, dieselpowered truck is used and an average distance of $1287 \mathrm{~km}(800 \mathrm{mi})$ is travelled. This distance was estimated by De Kleine (2009) and is based on five manufacturing locations of major residential air conditioner manufacturers.

It is assumed that a qualified service technician comes to the building site to check and service the unit every three years to ensure optimal performance and lifetime. It is assumed that the qualified technician is within a $24 \mathrm{~km}(15 \mathrm{mi})$ service radius. This distance is driven in a gasoline-powered van and is shared amongst other service visits for that technician, assuming that the same technician is making more than one service call during that trip. Assuming the technician makes 5 service calls in one day, one-fifth of the impacts from driving $24 \mathrm{~km}$ (15 mi) are allocated to the product, or $4.8 \mathrm{~km}$ (3 mi). Data for a van come from ecoinvent. Unplanned service visits (i.e., unanticipated issues that require a service technician) are not included in the modeling assuming that the home owner adequately follows the maintenance and care guidelines.

A lifetime of 15 years has been assumed for the air conditioners (National Association of Home Builders (NAHB) Research Center 2007). During use, refrigerant is assumed to escape at a rate of $2 \%$ per year of the total refrigerant (De Kleine 2009). It is recharged every three years during the maintenance visit.

\subsubsection{Air-Source and Water-Source Heat Pumps}

The BIRDS database includes both air-source and water-source heat pumps. Heat pumps are heating systems that transfers heat from air, water, or ground sources to air for space heating (or in reverse for cooling) in a building. The air source heat pump, also called air-to-air heat pump, uses a fan to extract lower grade heat from the air while the water source, also called water-to-air heat pump, uses a heat collector. Both systems have a heat pump unit with an evaporator, a compressor and a condenser.

\subsection{Upstream Materials Production through Manufacturing}

\section{Air-Source Heat Pump}

No manufacturer-specific data was available, so publicly-available sources of data for the bill of materials and manufacturing were used as a proxy and scaled according to weights of actual products on the market. For the air-source heat pump, a 2-ton (24 $000 \mathrm{Btu} / \mathrm{hr}$ ) Nutone Q7RF packaged heat pump was assumed to be a representative product since its size is appropriate for use in a small office building. ${ }^{30}$

\footnotetext{
${ }^{30}$ Product information can be found at: http://www.nutonehvac.com/NuTone-Q7RF-Packaged-Heat-Pumpp/q7rf.htm. NIST does not endorse any manufacturer-specific product listed in this document.
} 
Data for the bill of materials and manufacturing (specifically the assembly process) came from Table 2 of Greening (2012), a study evaluating the life cycle of domestic heat pumps in the UK. The mass of materials of the Greening (2012) air-source heat pump was scaled down to the mass of the Nutone unit, i.e., $214 \mathrm{~kg}$ (472 lb) to $117.5 \mathrm{~kg}$ (259 lb), and shipping weight relative to total unit weight was obtained from Nutone (2017). The $9.5 \mathrm{~kg}$ (21.0 lb) of shipping weight was used as packaging mass, which includes wood frame/boards, metal strapping, and poly strapping. The data modeled for BIRDS are as follows:

\section{Table 5-45 Air-Source Heat Pump Bill of Materials}

\begin{tabular}{llccl} 
& & \multicolumn{2}{c}{ Mass } & Notes \& Assumptions \\
Parts \& Components & Materials & $\mathbf{k g}$ & $\mathbf{l b}$ & \\
Bill of Materials & & & & \\
Evaporator and & Low-alloyed steel & 17.6 & 38.7 & \\
Housing and & Reinforcing steel & 65.9 & 145.2 & \\
Wiring, piping and & Copper & 19.3 & 42.6 & \\
Pipework insulation & Elastomer & 8.8 & 19.4 & As polybutadiene \\
Wiring insulation & PVC & 0.9 & 1.9 & \\
Lubricating oil & Polyolester oil & 1.5 & 3.3 & \\
Air fan & Copper & 0.8 & 1.7 & \\
& HDPE & 0.3 & 0.6 & Initial charge in the unit. Model \\
Refrigerant & R-410A & 2.4 & 5.3 & includes 3\% loss at manuf. \\
& & & & \\
Total BOM & & $\mathbf{1 1 7 . 2}$ & $\mathbf{2 5 8 . 4}$ & \\
& & & & \\
Manufacturing & & & & \\
Electricity & MJ (kWh) & 276.7 & $(76.9)$ & \\
Natural gas & MJ (mmBtu) & 768.6 & $(0.728)$ & \\
& & & & \\
Packaging & & & & \\
Wood frame/boards & Wood & 8.1 & 17.8 & \\
Metal strapping & steel & 0.95 & 2.1 & \\
Plastic protective film & LDPE & 0.48 & 1.0 & \\
\hline
\end{tabular}

\section{Water-Source Heat Pump}

No manufacturer-specific data was available, so publicly-available sources of data for the bill of materials and manufacturing were used as a proxy and scaled according to weights from actual products on the market. For the water-source heat pump, a vertical stack water-source heat pump with a 2-ton (24 $000 \mathrm{Btu} / \mathrm{hr}$ ) rating was used, specifically the Daikin cabinet Model WHVF with mass of $76.2 \mathrm{~kg}$ (168 lb) and chassis Model WVHC with mass of $66.2 \mathrm{~kg}$ (146 lb) (Daikin 2017).

The data used for the bill of materials and manufacturing came from Table 2 of Greening (2012). The mass of materials of the water-source heat pump system were scaled to the slightly larger mass of the Daikin unit, i.e., $133 \mathrm{~kg}$ (293 lb) scaled to $142 \mathrm{~kg}$ (314 lb), the total weight of the chassis and cabinet. Daikin (2017) also provided shipping weight that was used to approximate

\footnotetext{
31 The unit in Greening (2012) used R-134a. The refrigerant used by the Nutone unit, R-410a, is modeled here.
} 
packaging. The chassis packaging, $12.5 \mathrm{~kg}$ (27.5 lb), includes skids/pallet, cardboard, metal straps, and plastic wrap. The cabinet packaging, $13.3 \mathrm{~kg}$ (29.2 lb), consists of lumber framing to protect the product during shipping. The data modeled for BIRDS are as follows:

Table 5-46 Water-Source Heat Pump Bill of Materials

\begin{tabular}{|c|c|c|c|c|}
\hline \multirow[b]{2}{*}{ Parts \& Components } & \multirow[b]{2}{*}{ Materials } & \multicolumn{2}{|c|}{ Mass } & \multirow{2}{*}{ Notes \& Assumptions } \\
\hline & & Kg & Lb & \\
\hline \multicolumn{5}{|l|}{ Bill of Materials } \\
\hline Evaporator and condenser & Low-alloyed steel & 21.45 & 47.3 & \\
\hline Housing and compressor & Reinforcing steel & 80.44 & 177.3 & \\
\hline $\begin{array}{l}\text { Wiring, piping and } \\
\text { expansion valve }\end{array}$ & Copper & 2360 & 50 & \\
\hline Pipework insulation & Elastomere & $\begin{array}{l}23.60 \\
1073\end{array}$ & $\begin{array}{l}52.0 \\
23.6\end{array}$ & As polybutadiene \\
\hline Wiring insulation & PVC & $\begin{array}{c}10 . / 3 \\
1.07\end{array}$ & $\begin{array}{l}2.0 \\
2.4\end{array}$ & \\
\hline Lubricating oil & Polyolester oil & 1.82 & 4.0 & \\
\hline Refrigerant $^{32}$ & $\mathrm{R}-410 \mathrm{~A}$ & 3.31 & 7.3 & $\begin{array}{l}\text { Initial charge in the unit. Model } \\
\text { includes } 3 \% \text { loss at manuf. }\end{array}$ \\
\hline Total BOM & & 142.43 & 314.0 & \\
\hline \multicolumn{5}{|l|}{ Manufacturing } \\
\hline Electricity & MJ (kWh) & 361 & $(100.4)$ & \\
\hline Natural gas & MJ (mmBtu) & 939 & $(0.889)$ & \\
\hline \multicolumn{5}{|l|}{ Chassis Packaging } \\
\hline Pallet & Wood & 6.1 & 13.5 & \\
\hline Corrugated cardboard box & Corrugated board & 6.1 & 13.5 & \\
\hline Metal strapping & Steel & 0.12 & 0.27 & \\
\hline Plastic film & LDPE & 0.13 & 0.29 & \\
\hline $\begin{array}{l}\text { Cabinet Packaging } \\
\text { Wood framing/boards }\end{array}$ & Wood & 13.3 & 29.2 & \\
\hline
\end{tabular}

Data for the vertical pipes for the return, supply and condensate risers were included with the water source unit heater model. ${ }^{33}$ Water use in the supply pipe and the heat rejection fluid, usually propylene glycol and/or ethylene glycol, was excluded, since the number of floors in the building and heat pump usage are a variable and these data could not be appropriately apportioned to "one floor” of pipe data in this model. For $3.05 \mathrm{~m}$ (10 ft) and $5.1 \mathrm{~cm}$ (2 in) diameter copper pipes, the following was modeled:

\footnotetext{
${ }^{32}$ The unit in Greening (2012) used R-134a. The refrigerant used by the Daikin unit, R-410a, is modeled here. ${ }^{33}$ Calculated based on the Daikin (2017) specifications and drawings for Supply, Return, and Condensate Risers. Generic specifications from Mid-States Supply (2018).
} 
Table 5-47 Water-Source Heat Pump Piping Bill of Materials

\begin{tabular}{|c|c|c|c|}
\hline \multirow[b]{2}{*}{ Materials } & \multicolumn{2}{|c|}{ Mass } & \multirow[t]{2}{*}{ Notes \& Assumptions } \\
\hline & Kg & Lb & \\
\hline Copper pipe & 23.8 & 52.5 & All three pipes \\
\hline Polyethylene insulation & 0.19 & 0.43 & \\
\hline Brass & 16.6 & 36.6 & \\
\hline Total & 40.6 & 89.6 & For $3.05 \mathrm{~m}(10 \mathrm{ft})$ \\
\hline
\end{tabular}

\subsection{Materials Data}

The steel parts in the units are modeled using World Steel Association (2011) data provided in the Industry 2.0 database within SimaPro. Copper LCA data from International Copper Association (ICA) (2012) is used for the copper components in the units. Polybutadiene is modeled as the elastomer pipework insulation (Ecoinvent 2018). PVC for the wiring insulation and HDPE comes from NREL (2015). The lubricant comes from Ecoinvent (2018). R-410a data are based on a 50/50 share of difluoromethane (R-32) and pentafluoroethane (R-125). Due to lack of available production data on both chemicals, proxies were selected: Trifluoromethane (HFC-23) for difluoromethane and 1,1, difluoroethane (HFC 152a) for R125. While proxies were used for the production aspect of the chemicals, any release of these was based on the release of R-32 and R-125, not the proxy chemicals, so that ozone depletion impact remains zero and global warming potential impact is calculated appropriate to R-410A. For the vertical piping, copper data come from International Copper Association (ICA) (2012), brass comes from Ecoinvent (2018), and polyethylene comes from NREL (2015).

Packaging of these systems include wood frame/boards, wood pallets, corrugated board, metal strapping (assumed to be steel), and poly strapping (assumed to be high density polyethylene). The wood, modeled as planed dried hardwood, and HDPE come from NREL (2015). Steel comes from World Steel Association (2011). Corrugated board comes from Ecoinvent (2018).

Raw materials are transported to the manufacturing plant via diesel truck an assumed average distance of $805 \mathrm{~km}$ (500 mi) (NREL 2015).

\subsection{Transportation to the Building Site through End-of-Life}

Transportation of the heat pumps to the building site is modeled an assumed average of $644 \mathrm{~km}$ (400 mi) by heavy-duty diesel fuel-powered truck. These are modeled as generic products manufactured in many locations around the U.S., reducing the overall distance transported.

Table 2 in Greening (2012) estimates an annual refrigerant loss of $6 \%$ during operation, for both systems. This is treated in the model as released to air and being replenished by a technician. For the air-source heat pump, the annual amount is $0.14 \mathrm{~kg}(0.32 \mathrm{lb})$, and for the water-source heat pump, it is $0.2 \mathrm{~kg}(0.44 \mathrm{lb})$. A qualified service technician is modeled as coming to the building site one time per year to replenish refrigerant and check and service the units to optimize performance and lifetime. It is assumed that the technician is within a $24 \mathrm{~km}$ (15 mi) service 
radius. This distance, driven in a gasoline-powered van, is shared amongst other service visits for that technician, assuming the same technician is making more than one service call during that trip. Assuming the technician makes 5 service calls in one day, one-fifth of the impacts from driving $24 \mathrm{~km}$ (15 mi) are allocated to the product, or $4.8 \mathrm{~km}$ (3 mi). Data for a van come from Ecoinvent (2018). Unplanned service visits (i.e., unanticipated issues that require a service technician) are not included in the modeling assuming that the building manager follows HVAC maintenance and care guidelines.

The water source heat pump uses an air filter that the building owner is assumed to change three times per year. In BIRDS it is modeled as a disposable filter that is purchased locally. The filter that has been modeled for this system is based on a MERV 11 filter of size $22.9 \times 27.9 \times 2.54 \mathrm{~cm}$ (9 x $11 \times 1$ in) (P/N 4030671), shown in Table 5-48. The bills of materials have been adjusted to the size of the filter used by the water source heat pump (50.8 x 76 x $2.54 \mathrm{~cm}(24 \times 30 \times 1 \mathrm{in})$ ), or a factor of 6.1 .

\section{Table 5-48 Filter Size 9x11x1 Bill of Materials}

\begin{tabular}{lccl} 
& \multicolumn{2}{c}{ Mass } & \\
Part and/or Material & $\mathbf{l b}$ & $\mathbf{g}$ & Notes \\
Plastic stretch wrap (packaging) & 0.002 & 1.0 & LDPE \\
Cardboard "frame" & 0.08 & 37.0 & Bleached \\
Rubber/plastic in the pleats & 0.12 & 55.0 & 11 rows, 5 g per row \\
Synthetic fiber & 0.075 & 34.0 & Assume PET \\
Total & $\mathbf{0 . 2 8}$ & $\mathbf{1 2 7}$ & \\
\hline
\end{tabular}

The data for the LDPE, polypropylene pleats, and the synthetic fiber, assumed to be a spunbonded polyethylene terephthalate (PET), come from NREL (2015). Data for the spunbonding process - $0.76 \mathrm{kWh}$ (2.73 MJ) per kg - come from EA (2011), Table 4.2. Ecoinvent (2018) provides the data for the cardboard. Filters are landfilled after they are replaced.

Lifetimes of 15 and 19 years have been assumed for the air-source and water-source heat pumps, respectively (ASHRAE Undated). At the end-of-life, it is assumed that the heat pumps are sent for recycling to recover valuable metal. What cannot be recovered is modeled as landfilled. A distance of $48 \mathrm{~km}$ (30 mi) to the landfill in a heavy-duty diesel truck has been modeled. The landfill is based on waste management process data from Ecoinvent (2018). Eighty percent of the refrigerant is assumed to be recovered and reused, while $20 \%$ is lost to the air at end of life.

\subsubsection{Gas-Fired Unit Heater}

A gas-fired unit heater of capacity between $138000 \mathrm{Btu} / \mathrm{hr}(40517 \mathrm{~W}$ ) to $541000 \mathrm{Btu} / \mathrm{hr}$ (158 $500 \mathrm{~W}$ ) was included in the BIRDS database for applications such as a standalone retail store or warehouse. No publicly available data on the bill of materials or manufacturing of such a system was available. Data were calculated using specifications for a product on the market deemed appropriate as a proxy for the desired application in BIRDS: Trane 350000 Btu/hr 
(87 $800 \mathrm{~W}$ ) tubular propeller fan gas unit heater (Trane 2014). ${ }^{34}$ Publicly available literature on this unit provided adequate specifications on the system's components, enabling the detailed compilation of the bill of materials. Table 5-49 presents a description of the system's subcomponents/parts that was developed using a combination of information from literature and expert judgement. Using published surface areas, material densities and known or estimated gauges, these data were compiled into the bill of materials in Table 5-49.

Table 5-49 Gas-fired Unit Heater Components and Parts

$\begin{array}{ll}\text { Component } & \text { Notes and assumptions on Subcomponents / Parts / Materials } \\ \text { Cabinet } & 20 \text { gauge cold rolled steel }(40.6 \mathrm{~kg}), \text { powder coating }(0.5 \mathrm{~kg}) \\ \text { Fan blades } & \text { Aluminum }(0.29 \mathrm{~kg}) \\ \text { Motor } & 6.3 \mathrm{~kg} \text {, made up of steel, copper, \& polyethylene parts } \\ \text { Breakers, circuitry, etc. } & 0.1 \mathrm{~kg} \text { (estimated) } \\ \text { Copper wiring } & 0.66 \mathrm{~kg} \text { (estimated) } \\ \text { Wiring insulation } & 0.26 \mathrm{~kg} \text { (estimated) } \\ \text { Heat exchanger tubes, burner, etc. } & \text { Aluminized steel }(96.2 \mathrm{~kg})\end{array}$

Table 5-50 presents the aggregated bill of materials totaling the published weight of the Trane unit (145 kg (319.7 lb)), with total mass of packaging calculated using the shipping and product weight difference (Trane (2014), Table 20).

Table 5-50 Gas-fired Unit Heater Bill of Materials

\begin{tabular}{lcc}
\multicolumn{1}{c}{ Material / component description } & \multicolumn{2}{c}{ Mass } \\
Aluminized steel & Kg & Lb \\
Cold rolled sheet & 96.23 & 212.2 \\
Cast steel & 40.61 & 89.5 \\
Copper wiring & 3.87 & 8.52 \\
Aluminum sheet & 2.66 & 5.87 \\
PVC wiring insulation & 0.29 & 0.64 \\
Polyethylene & 0.26 & 0.58 \\
Powder coating & 0.47 & 1.03 \\
Breakers, circuitry & 0.51 & 1.13 \\
Total & 0.10 & 0.22 \\
Packaging & $\mathbf{1 4 5 . 0}$ & $\mathbf{3 1 9 . 7}$ \\
Lumber (pallet shared) & & \\
Corrugated board & 11.2 & 24.7 \\
Metal strapping & 16.0 & 35.2 \\
Total & 0.84 & 1.9 \\
\hline
\end{tabular}

${ }^{34}$ NIST does not endorse any manufacturer-specific product listed in this document. 
Data for the cabinet steel, and heat exchanger tubes and burner come from data on steel semifabricated products in the Industry 2.0 database within SimaPro (World Steel Association 2011) and customized to North American production. The powder coating on the cabinet comes from Ecoinvent (2018). Aluminum fan blades data are based on Aluminum Association's industryaverage LCA data provided by Ecoinvent (2018). Copper comes from (International Copper Association (ICA) 2012). Breakers and circuitry electronic parts were modeled as surface mounted, $\mathrm{Pb}$ free printed wiring board from Ecoinvent (2018).

The motor is $61 \%$ steel, $32 \%$ copper, and $7 \%$ polyethylene components (Nyman 2005). Steel in the motor is cold rolled coil (World Steel Association 2011). Copper wire and copper in the motor are based on International Copper Association (ICA) (2012). The low-density polyethylene resin (LDPE) in the motor and the PVC wiring insulation come from NREL (2015).

Lacking availability of data on manufacturing, assembly data for a $63 \mathrm{~kg}$ (139 lb) fan coil unit was used as proxy (Churcher 2014) and scaled up to the size of the unit heater, amounting to 226 MJ (62.7 kWh) electrical energy for assembly and 75.3 MJ (71 $302 \mathrm{Btu}$ ) natural gas for other processes and overhead heating use (NREL 2015).

Packaging is modeled as units enclosed in corrugated board (Ecoinvent 2018) and tied with metal strapping (World Steel Association 2011) transported on shared pallets (lumber from NREL (2015)).

\section{Transportation to the Building Site through End-of-Life}

Transportation to the building site is modeled as a generic product that could be manufactured in many locations around the U.S., using an average of $644 \mathrm{~km}$ (400 mi) by heavy-duty diesel fuelpowered truck.

A qualified service technician is assumed to be located within a $24 \mathrm{~km}$ (15 mi) service radius and visits the building site one time per year to ensure the unit performance and lifetime is maintained. This distance, driven in a gasoline-powered van, is assumed to be shared amongst other service visits for that technician. Assuming the technician makes 5 service calls in one day, one-fifth of the impacts from driving $24 \mathrm{~km}(15 \mathrm{mi})$ are allocated to the product, or $4.8 \mathrm{~km}$ (3 mi). Data for a van come from Ecoinvent (2018). Unplanned service visits (i.e., unanticipated issues that require a service technician) are not included in the modeling assuming that the building manager follows HVAC maintenance and care guidelines.

A lifetime of 13 years has been assumed for this unit(ASHRAE Undated). At the end-of-life, it is assumed that it is sent for recycling to recover valuable metals with the remainder that cannot be recovered is landfilled assuming a heavy-duty diesel truck transporting the waste $48 \mathrm{~km}$ (30 mi) to the landfill. The landfill is based on waste management process data in Ecoinvent (2018). 


\subsubsection{Fluid Cooler}

A fluid cooler was included in the BIRDS database to use with the water source heat pump in an apartment high-rise or large office building. No publicly available data on the bill of materials or manufacturing of such a system was found for this item, so data were estimated and calculated using specifications for a product on the market deemed appropriate and reasonable for the desired application in BIRDS.

\subsection{Upstream Materials Production through Manufacturing}

The Johnson Controls VDCF Air-Cooled Fluid Cooler with a capacity of 240 tons (2890 MBtu/hr) was chosen as it is reasonable for the intended application, and enough publicly available literature on this unit provided adequate specifications on the system's components, enabling the detailed compilation of the bill of materials. The fluid cooler modeled has two rows of six fans with 2-HP motors. Table 5-51 presents a description of the system's subcomponents/parts. Some information was obtained from Johnson Controls (2015) while others were based on expert opinion. Using published surface areas, material densities and known or estimated gauges, these data were compiled into the bill of materials in the table that follows.

\section{Table 5-51 Air-Cooled Fluid Cooler Components and Parts}

\begin{tabular}{ll} 
Component & Details \\
Motor & $3.6 \mathrm{~kg}$ - $64 \%$ steel, $8.9 \%$ aluminum, 23.6 \% copper, and 3.4 \% polyethylene* \\
Motor & $3.17 \mathrm{~kg}$ - Steel, Al, Cu, polyethylene \\
2 -HP motors (12 total)** & $240 \mathrm{~kg}-$ Steel, Al, Cu, polyethylene \\
Legs & $20 \mathrm{~kg}-$ Steel \\
Casing & $487 \mathrm{~kg}-$ Steel \\
Copper coils & $567 \mathrm{~kg} 16 \mathrm{~mm}$ OD \\
Fins & $1509 \mathrm{~kg}-$ aluminum \\
Support channels (12 total) & $23 \mathrm{~kg}$ - Galvanized steel \\
Breakers, circuitry etc. & $1.0 \mathrm{~kg}$ (estimate) \\
Copper wiring & $40 \mathrm{~kg}$ (estimate) \\
Wiring insulation & $10 \mathrm{~kg}$ (estimate) - assume PVC \\
Fan blades & $83 \mathrm{~kg}$ (estimate) - aluminum \\
\hline * Nyman (2005) & \\
** IronHorse (2017) & \\
\hline
\end{tabular}

The following table presents the bill of materials totaling the published weight of the Johnson Controls unit (2 979 kg (6 568 lb)) (Johnson Controls 2015). Packaging was not included in the model as packaging mass is assumed to be less than $1 \%$ of the total mass of the product. 


\section{Table 5-52 Fluid Cooler Bill of Materials}

\begin{tabular}{lcc} 
Material / component & \multicolumn{2}{c}{ Mass } \\
description & kg & lb \\
Galvanized steel & 530.5 & 1169.5 \\
Cast steel & 153.5 & 338.4 \\
Aluminum sheet & 1591.3 & 3508.2 \\
Aluminum casting & 21.3 & 46.9 \\
Copper tube & 566.7 & 1249.3 \\
Copper Wiring & 96.7 & 213.1 \\
PVC wiring insulation & 10.0 & 22.0 \\
Polyethylene & 8.3 & 18.2 \\
Breakers, circuitry etc. & 1.0 & 2.2 \\
Total & $\mathbf{2 9 7 9 . 1}$ & $\mathbf{6 5 6 7 . 8}$ \\
\hline
\end{tabular}

The steel parts in the units are modeled using World Steel Association (2011) data provided in the Industry 2.0 database within SimaPro. Aluminum industry-wide LCA data are provided in Ecoinvent (2018). International Copper Association (ICA) (2012) data were used for the copper tube and wiring. NREL (2015) provided data for PVC and polyethylene. Breakers and circuitry electronic parts were modeled as surface mounted, $\mathrm{Pb}$ free printed wiring board from Ecoinvent (2018).

No data were available for assembly of this unit, assembly was modeled as an assumed $1 \%$ of the aggregated impacts of the bill of materials and parts forming stage. The rationale is that many of the fluid cooler's components, including the motors, aluminum fins, fan blades, and electronic parts, come to the plant ready to be assembled into final products. The bill of materials stage already includes the parts forming for the individual components listed above, so that aspect of product manufacturing has already been included upstream. Assembly is thus assumed to be a small proportion of materials production and parts forming.

\subsection{Transportation to the Building Site through End-of-Life}

Transportation of the unit to the building site is modeled an assumed average of $804.5 \mathrm{~km}$ (800 mi) by heavy-duty diesel fuel-powered truck.

Water use and heat rejection fluid, usually propylene glycol and/or ethylene glycol, was excluded, since the number of floors in the building and number of heat pump units per floor vary based on BIRDS user input, thus total water-cooling usage could not be appropriately apportioned to "one floor" of data for this model. Still, energy use during use phase is included as a separate calculation within BIRDS. A qualified service technician is modeled as coming to the building site two times per year to ensure the unit is optimized for performance and lifetime, and heat rejection fluid is at the appropriate level. It is assumed that the technician is within a 24 km (15 mi) service radius. This distance, driven in a gasoline-powered van, is shared amongst other service visits for that technician, assuming that the same technician is making more than one service call during that trip. Assuming the technician makes 5 service calls in one day, onefifth of the impacts from driving $24 \mathrm{~km}(15 \mathrm{mi})$ are allocated to the product, or $4.8 \mathrm{~km}$ (3 mi). Data for a van come from Ecoinvent (2018). Unplanned service visits (i.e., unanticipated issues 
that require a service technician) are not included in the modeling assuming that the building manager follows HVAC maintenance and care guidelines.

A lifetime of 20 years has been assumed for this unit (ASHRAE Undated). At the end-of-life, it is assumed that it is sent for recycling to recover valuable metals. What cannot be recovered is modeled as landfilled. A distance of $48 \mathrm{~km}$ (30 mi) to the landfill in a heavy-duty diesel truck has been modeled. The landfill is based on waste management process data in Ecoinvent (2018).

\subsubsection{Electric Cabinet Heater}

The BIRDS database includes an electric cabinet heater that is used to heat a hotel room. The cabinet heater, with a coil heat exchanger and fan assembly, is considered to be a type of a fan coil unit. While fan coil units come in many sizes and configurations, they include a heat exchanger and fan which are used to control temperature in the space where they are used.

\subsection{Upstream Materials Production through Manufacturing}

No manufacturer-specific data was available, so publicly-available sources of data for the bill of materials and manufacturing were used as a proxy and scaled according to the weight from an actual product on the market. The vertical cabinet Force-Flo Cabinet Heater size 060 by Trane was used to represent this product since its size is appropriate for use in a hotel room, and product literature provided the weight of packaging and product, to use for the bill of materials for this product in BIRDS (Trane 2000).

Data for the bill of materials and manufacturing of a fan coil unit came from Figure 5 and Figure 6 of Churcher (2014), part of a larger European Union study on HVAC systems (Churcher 2014). The mass of materials of the Churcher fan coil unit and the manufacturing data were scaled down to the mass of the Trane unit, i.e., $69.3 \mathrm{~kg}(152.8 \mathrm{lb})$ to $63.0 \mathrm{~kg}$ (139 lb). Trane's reported shipping weight relative to total mass, a difference of $7 \mathrm{~kg}$ (15.4 lb)(Churcher 2014), was used as packaging mass, which is assumed to be comprised of corrugated board, steel strapping and plastic film. The data modeled for BIRDS are as follows: 
Table 5-53 Cabinet Heater Bill of Materials

\begin{tabular}{|c|c|c|c|}
\hline \multirow[b]{2}{*}{ Materials/Parts } & \multicolumn{2}{|c|}{ Mass } & \multirow[t]{2}{*}{ Notes \& Assumptions } \\
\hline & Kg & Lb & \\
\hline \multicolumn{4}{|l|}{ Bill of Materials } \\
\hline Galvanized steel sheet & 37.78 & 83.3 & Internal skeleton, housing, fan \\
\hline Cast iron & 2.39 & 5.3 & Fan motor is iron \& copper \\
\hline Copper tube & 8.13 & 17.9 & $10 \mathrm{~mm}$ \\
\hline Copper wiring & 2.27 & 5.0 & \\
\hline Insulation: polyurethane foam & 0.29 & 0.6 & \\
\hline Aluminum sheet & 8.61 & 19.0 & $0.2 \mathrm{~mm}$ \\
\hline Control valves: brass & 2.86 & 6.3 & \\
\hline РCB & 0.48 & 1.1 & \\
\hline Total BOM & 63.0 & 138.9 & \\
\hline Filter & 0.24 & 0.53 & $14.0 \mathrm{~cm}$ x $85.3 \mathrm{~cm} \mathrm{x} 2.54 \mathrm{~cm}$ \\
\hline Packaging & & & Materials and quantities assumed \\
\hline Corrugated box & 6.7 & 14.7 & \\
\hline Metal strapping (steel) & 0.25 & 0.54 & \\
\hline Plastic protective film - low density & 0.11 & 0.23 & \\
\hline \multicolumn{4}{|l|}{ Manufacturing } \\
\hline Electricity & 98 & (27.3) & \\
\hline Natural gas & 32.7 & (30 979) & \\
\hline
\end{tabular}

The steel parts in the units are modeled using World Steel Association (2011) data provided in the Industry 2.0 database within SimaPro. Aluminum industry-wide LCA data are provided in Ecoinvent (2018). International Copper Association (ICA) (2012) data were used for the copper tube and wiring. LDPE data came from NREL (2015). Polyurethane foam and brass came from Ecoinvent (2018). The PCB was modeled as surface mounted, $\mathrm{Pb}$ free printed wiring board from Ecoinvent (2018).

Corrugate packaging data come from Ecoinvent (2018); metal strapping is based on World Steel Association (2011) data, and the LDPE comes from NREL (2015). Manufacturing data come from NREL (2015).

Raw materials are transported to the manufacturing plant via diesel truck with an assumed average distance of $805 \mathrm{~km}$ (500 mi) (NREL 2015).

\subsection{Transportation to the Building Site through End-of-Life}

Transportation of the unit to the building site is modeled an assumed average of $644 \mathrm{~km}$ (400 mi) by heavy-duty diesel fuel-powered truck. This is modeled as a generic product that could be manufactured in many locations around the U.S., reducing the overall distance transported.

The cabinet heater uses an air filter that the building owner is assumed to change two times per year. In BIRDS it is modeled as a disposable filter that is purchased locally. The filter that has been modeled for this system is based on a MERV 11 filter of size 22.9 × 27.9 × $2.54 \mathrm{~cm}(9 \times 11$ $\mathrm{x} 1 \mathrm{in})(\mathrm{P} / \mathrm{N}$ 4030671), shown in Table 5-54. The bills of materials have been adjusted to the size of the filter used by the Trane cabinet heater $(14.0 \mathrm{~cm}$ x $85.3 \mathrm{~cm}$ x $2.54 \mathrm{~cm}$ (5.5 in x 33.6 in x 1 in)), or a factor of 1.9. 
Table 5-54 Filter Size 9x11x1 Bill of Materials

\begin{tabular}{lccl} 
& \multicolumn{2}{c}{ Mass } & \\
Part and/or Material & Lb & Gram & Notes \\
Plastic stretch wrap (packaging) & 0.002 & 1.0 & LDPE \\
Cardboard "frame" & 0.08 & 37.0 & Bleached \\
Rubber/plastic in the pleats & 0.12 & 55.0 & 11 rows, 5 g per row \\
Synthetic fiber & 0.075 & 34.0 & Assume PET \\
Total & $\mathbf{0 . 2 8}$ & $\mathbf{1 2 7}$ & \\
\hline
\end{tabular}

The data for the LDPE, polypropylene pleats, and the synthetic fiber, assumed to be a spunbonded polyethylene terephthalate (PET), come from NREL (2015). Data for the spunbonding process - $0.76 \mathrm{kWh}$ (2.73 MJ) per $\mathrm{kg}$ - come from EA (2011), Table 4.2. Ecoinvent (2018) provides the data for the cardboard. Filters are landfilled after they are replaced.

A qualified service technician is modeled as coming to the building site one time per year to ensure the unit is optimized for performance and lifetime. It is assumed that the technician is within a $24 \mathrm{~km}$ (15 mi) service radius. This distance, driven in a gasoline-powered van, is shared amongst other service visits for that technician, assuming the same technician is making more than one service call during that trip. Assuming the technician makes 5 service calls in one day, one-fifth of the impacts from driving $24 \mathrm{~km}(15 \mathrm{mi})$ are allocated to the product, or $4.8 \mathrm{~km}$ (3 mi). Data for a van come from Ecoinvent (2018). Unplanned service visits (i.e., unanticipated issues that require a service technician) are not included in the modeling assuming that the building manager follows HVAC maintenance and care guidelines.

A lifetime of 20 years has been assumed for this unit (ASHRAE Undated). At the end-of-life, it is assumed that it is sent for recycling to recover valuable metal. What cannot be recovered is modeled as landfilled. A distance of $48 \mathrm{~km}$ (30 mi) to the landfill in a heavy-duty diesel truck has been modeled. The landfill is based on waste management process data from Ecoinvent (2018).

\subsubsection{Lighting}

Two approaches are used to estimate the LCIA data for lighting. For residential buildings (apartments), light bulbs are replaced with higher efficiency bulbs while assuming the lighting system and number of fixtures remain constant. In the case of all commercial buildings, the lighting system is adjusted by increasing or decreasing wattage of the lighting system. All commercial buildings assume T-12 linear fluorescent fixtures because that is the assumed lighting system for each RS Means baseline building cost estimate.

For residential buildings, data for three lighting alternatives are available in BIRDS: incandescent, compact fluorescent lamp (CFL), and light-emitting diode (LED). These were calculated on a per one $\mathrm{W} / \mathrm{h}$ basis so that BIRDS could calculate the energy needed for lighting based on time used. Figure 5-9 presents the lighting system boundaries for a CFL example. 


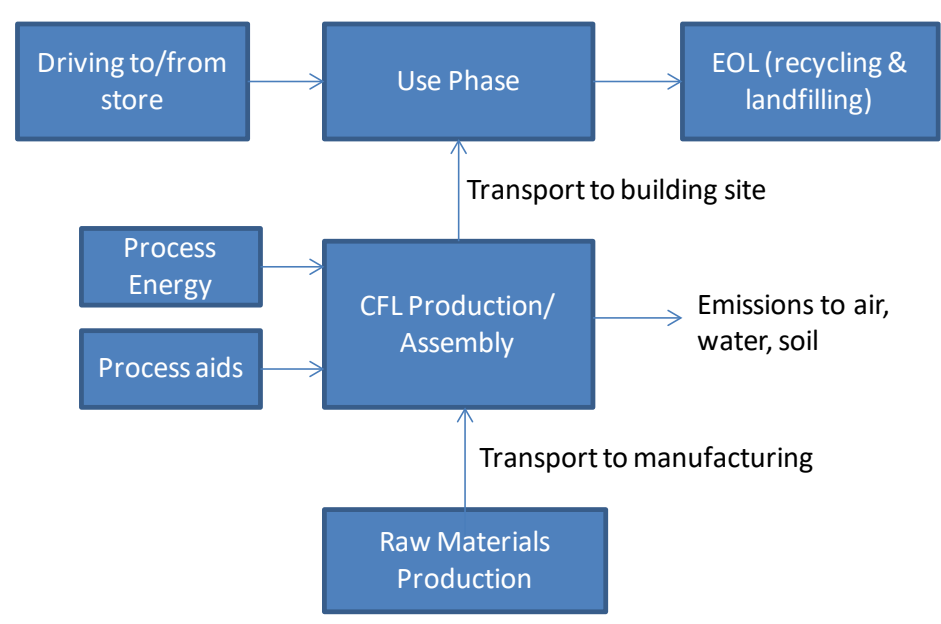

\section{Figure 5-9 Lighting System Boundaries - CFL Example}

To model the lighting alternatives based on functional equivalency, the model must account for an equivalent or comparable lumen output, i.e., measure of brightness. The lighting characteristics from Energy Efficiency and Renewable Energy (EERE) (2012) were used to define the BIRDS’ lighting systems' functional equivalency. Table 4.1 in Energy Efficiency and Renewable Energy (EERE) (2012), reproduced in part in Table 5-55, summarizes the average characteristics of the lamps analyzed within existing lighting LCA literature (at the time) in terms of lumen output, wattage, and lifetime. For the continuously-improving LED technology, a lumen output available in 2011 was used, which was higher than lumens from earlier studies.

\section{Table 5-55 Performance of Lighting Technologies in BIRDS}

\begin{tabular}{lcccc} 
Lamp Type & Watts & Lumens & $\begin{array}{c}\text { Operating } \\
\text { Lifetime (hr) }\end{array}$ & $\begin{array}{c}\text { Number of Equiv. } \\
\text { Systems }\end{array}$ \\
Incandescent & 60 & 900 & 1000 & 25 \\
CFL & 15 & 900 & 8500 & 2.9 \\
LED (2011) & 12.5 & 800 & 25000 & 1 \\
\hline
\end{tabular}

For residential buildings, the baseline RS Means cost estimate assumes incandescent light bulbs. In order to reduce the watts per unit of floor area to meet the different standard edition requirements, a fraction of the incandescent bulbs are assumed to be replaced by CFL bulbs. The assumed replacement rates are 1 year for incandescents and 7 years for CFLs.

For commercial buildings, three linear fluorescent lighting systems are included in the BIRDS commercial database: T5, T8, and T12. This nomenclature refers to tube circumference in centimeters. These lighting systems include the fluorescent bulbs; the ballast, which controls current; and the lamp fixture (housing, wiring, and reflector). The reference flow for the lighting systems in BIRDS is one Watt-hour (W/h) so that BIRDS calculates lighting energy for each product based on time used in a building over the 40-year study period. The model accounts for the number of bulb and ballast replacements over these 40 years; the fixture is assumed to not need replacement. The figure below presents the boundaries for the linear fluorescent lighting system. 


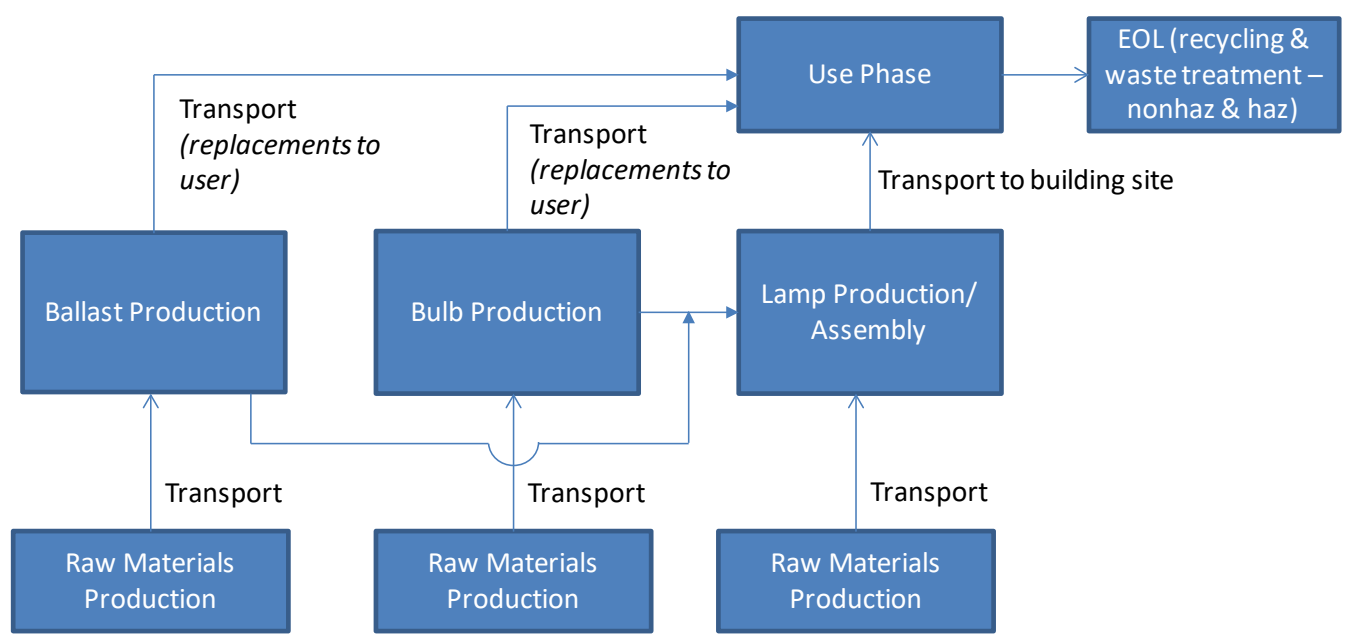

Figure 5-10 Linear Fluorescent Lighting System Boundaries

Lighting alternatives are modelled based on functional equivalency, accounting for an equivalent or comparable lumen output (i.e., measure of brightness). Comparable performance data for all three linear fluorescent products is based on products offered by a web-based company dedicated to selling light bulbs. Table 5-56 summarizes popular products offered for the three bulb sizes and their comparable lumens. Note that the lumens are similar enough in magnitude, although not equivalent, that these may be considered comparable for purposes of BIRDS.

Table 5-56 Linear Fluorescent Light Bulb Characteristics

$\begin{array}{lccc} & \text { T5 } & \text { T8 } & \text { T12 } \\ \text { Length cm (in) } & 117(46) & 122(48) & 122(48) \\ \text { Operating lifetime (hr) } & 25000 & 30000 & 12000 \\ \text { Lumens - nominal } & 2900 & 2950 & 2850 \\ \text { Watts } & 28 & 32 & 45 \\ \text { Luminous efficiency (lumen/W) } & 104 & 92 & 63 \\ \text { Note: Data taken for several popular products in each category, retrieved from } \\ \text { https://www.bulbs.com/ }\end{array}$

The total number of bulbs and ballasts used over the BIRDS 40-year service life are calculated using the lights' total operating hours per building type and operating lifetime data reported in Table 5-56. Two bulbs are assumed to be used in the lamp fixture modeled for BIRDS. Table 5-57 summarizes the number of replacements of each commercial fluorescent lighting system, assuming 2 bulbs per fixture and total operating hours for each BIRDS building. 
Table 5-57 Building Lighting Usage and Replacement Rates

\begin{tabular}{|c|c|c|c|c|c|c|}
\hline \multirow[b]{2}{*}{ Building Type } & \multirow{2}{*}{$\begin{array}{c}\text { Avg. Hrs. } \\
\text { Operation }(1 \text { yr })^{35}\end{array}$} & \multirow{2}{*}{$\begin{array}{l}\text { Total hrs } \\
\text { (40 yrs) }\end{array}$} & \multicolumn{3}{|c|}{ \# Bulbs - 2 per fixture (40 yrs) } & \multirow{2}{*}{$\begin{array}{c}\text { \# Ballasts - al } \\
\text { products (40 } \\
{\text { yrs })^{36}}^{36}\end{array}$} \\
\hline & & & T5 & T-8 & T-12 & \\
\hline Outpatient Healthcare & 3878 & 155120 & 12.4 & 10.3 & 25.9 & 3.9 \\
\hline Hotel (Large \& Small) & 3294 & 131760 & 10.5 & 8.8 & 22.0 & 3.3 \\
\hline Warehouse & 3213 & 128520 & 10.3 & 8.6 & 21.4 & 3.2 \\
\hline $\begin{array}{l}\text { School (Primary \& } \\
\text { Secondary) }\end{array}$ & 3898 & 155920 & 12.5 & 10.4 & 26.0 & 3.9 \\
\hline $\begin{array}{l}\text { Restaurant (Sit Down \& } \\
\text { Fast Food) }\end{array}$ & 5070 & 202800 & 16.2 & 13.5 & 33.8 & 5.1 \\
\hline Strip mall & 4295 & 171800 & 13.7 & 11.5 & 28.6 & 4.3 \\
\hline Standalone Retail & 3139 & 125560 & 10.0 & 8.4 & 20.9 & 3.1 \\
\hline Large Office & 2722 & 108880 & 8.7 & 7.3 & 18.1 & 2.7 \\
\hline Medium Office & 3139 & 125560 & 10.0 & 8.4 & 20.9 & 3.1 \\
\hline Small Office & 3636 & 145440 & 11.6 & 9.7 & 24.2 & 3.6 \\
\hline Apartment Mid Rise & 1971 & 78840 & 6.3 & 5.3 & 13.1 & 2.0 \\
\hline Apartment High Rise & 1932 & 77280 & 6.2 & 5.2 & 12.9 & 1.9 \\
\hline
\end{tabular}

\subsubsection{Upstream Materials Production through Manufacturing}

The bills of materials for all three alternatives come from U.K. Department for Environment (2009). These bills of materials are based on Ramroth (2008) but with more detail/aggregation provided.

\subsection{Linear Fluorescent Lighting System}

The fluorescent lighting systems' bills of material and manufacturing data provided in Tähkämö (2013) is used to represent the systems in BIRDS because it is the most comprehensive bills of material and manufacturing data for all three aspects of the linear fluorescent lighting (the bulbs, ballast and fixture), and is representative of a system found in industrial and commercial buildings. The criteria for choosing this data set over other publicly-available data on linear fluorescent lighting was (1) inclusion of all necessary components of the system and (2) the overall data quality (accounting for a combination of the temporal, technological, and geographical aspects of the collected data). While Tähkämö (2013) data are not based on North American production, they were based on relatively recent primary manufacturing and bill of materials data. Data for the fluorescent lights came from European Lamp Companies Federation along with researchers and industry experts (Tähkämö 2013). The luminaire bill of materials and assembly of the final luminaire came from a luminaire manufacturer, and ballast bill of materials came from the ballast manufacturer.

\footnotetext{
${ }^{35}$ Data provided by NIST to Four Elements and based on ASHRAE 90.1-2004.

${ }^{36}$ Ballast lifetime data, i.e., approximately 40000 hours per ballast, from Table 4.1 in U.K. Department for Environment, Food and Rural Affairs (Defra) (2009). Life-cycle Assessment of Ultra-Efficient Lamps.
} 


\section{Table 5-58 Fluorescent Bulb (Tube) Bill of Materials}

\begin{tabular}{lccc}
\multicolumn{1}{r}{ Material / component } & \multicolumn{2}{c}{ Data per 1 Bulb } & \\
& gram & ounce & Comments \\
Glass tube, borosilicate & 115 & 4.06 & \\
Aluminum & 3 & 0.11 & Assume primary \\
Mercury (liquid) & 0.005 & 0.0002 & \\
Argon (liquid) & 0.5 & 0.02 & \\
Triphosphor & 2.5 & 0.09 & $\begin{array}{l}\text { No LCA production data } \\
\text { available }\end{array}$ \\
Total & $\mathbf{1 2 1 . 0}$ & $\mathbf{4 . 3}$ & \\
& & & \\
Packaging & & & \\
Corrugated board & 25 & 0.88 & \\
Source: Tähkämö (2013)Figure 1 Inventory data and the life cycle stages of a fluorescent \\
lamp luminaire.
\end{tabular}

This data was first scaled from a $1.5 \mathrm{~m}$ (4.9 ft) T5 bulb evaluated in Tähkämö (2013) to the more common size T5 bulb in the U.S. (see above table), or a factor of 0.78 . Then this adjusted bulb was scaled to the other bulb sizes based on the relative tube diameters/circumferences, as shown below.

\section{Table 5-59 Fluorescent Light Bulb Scaling Factors}

\begin{tabular}{lccc} 
& T5 & T8 & T12 \\
Tube diameter cm (in) & $1.59(0.625)$ & $2.54(1.0)$ & $3.81(1.5)$ \\
Tube circumference cm (in) & $5.0(1.96)$ & $8.0(3.14)$ & $12.0(4.7)$ \\
Scaling factor from BIRDS T5 & & 1.6 & 2.4 \\
\hline
\end{tabular}

The ballast bill of materials is provided in the next table. 
Table 5-60 Electronic Ballast Bill of Materials and Production Data

\begin{tabular}{|c|c|c|c|}
\hline \multirow[t]{2}{*}{ Material / component } & \multicolumn{2}{|c|}{ Data per 1 Ballast } & \multirow[b]{2}{*}{ Comments } \\
\hline & Gram & ounce & \\
\hline Capacitor & 18 & 0.63 & Assume electrolytic type \\
\hline Transformer & 54 & 1.90 & \\
\hline Diode & 1.0 & 0.04 & \\
\hline Resistor & 4.0 & 0.14 & Assume surface-mounted \\
\hline Transistor & 1.0 & 0.04 & $\begin{array}{l}\text { Assume through-hole } \\
\text { mounting }\end{array}$ \\
\hline Integrated circuit, logic type & 0.16 & 0.01 & Assume logic type \\
\hline Printed circuit board & 21 & 0.74 & $\begin{array}{l}\text { Modeled as surface mounted } \\
\text { and lead free }\end{array}$ \\
\hline Steel & 140 & 4.94 & Cold rolled \\
\hline Plastic parts & 12 & 0.42 & $\begin{array}{l}\text { Assumed polyethylene } \\
\text { terephthalate (PET) }\end{array}$ \\
\hline Total & 251 & 8.86 & \\
\hline Manufacturing Energy & MJ & kWh & \\
\hline Electricity & 10.8 & 3.0 & \\
\hline
\end{tabular}

Data for the fixture is provided in the next table. As a simplified assumption, only the aluminum used for housing (i.e., $1352 \mathrm{~g}$ ) is scaled according to bulb size.

Table 5-61 Fixture Bill of Materials and Assembly Data

\begin{tabular}{|c|c|c|c|}
\hline \multirow[t]{2}{*}{ Material / component } & \multicolumn{2}{|c|}{ Data per Fixture } & \multirow[b]{2}{*}{ Comments } \\
\hline & gram & Ounce & \\
\hline Aluminum (production mix) & 1352 & 47.7 & Scaled based on bulb size \\
\hline Aluminum cast alloy & 254 & 8.96 & Modeled as AlMg3 \\
\hline Steel & 32 & 1.13 & As cold rolled steel \\
\hline Copper Wire & 3.0 & 0.11 & \\
\hline Cable, unspecified & 65 & 2.29 & \\
\hline Plastic parts & 116 & 4.09 & Assume polypropylene (PP) \\
\hline Silicone & 7.0 & 0.25 & \\
\hline Total & 1829 & 64.5 & \\
\hline \multicolumn{4}{|l|}{ Packaging } \\
\hline Corrugated board & 400 & 14.1 & \multirow[b]{2}{*}{$\begin{array}{l}\text { Assume low density } \\
\text { polyethylene (LDPE) }\end{array}$} \\
\hline Packaging film & 5.0 & 0.18 & \\
\hline Manufacturing/Assembly Energy & MJ & kWh & \multirow{2}{*}{$\begin{array}{l}\text { To assemble and package the } \\
\text { ballast, lamps, \& fixture }\end{array}$} \\
\hline Electricity & 86.4 & 24.0 & \\
\hline Heat & 118.8 & 33.0 & Assume natural gas \\
\hline
\end{tabular}


Most of the data to model the linear fluorescent systems’ parts and materials come from Ecoinvent (2018) and industry-average LCA data from organizations including International Copper Association. ${ }^{37}$ Aluminum data come from Ecoinvent (2018) and is based on Aluminum Association's industry-average LCA data. Steel data came from Industry 2.0 database within SimaPro, which includes data on steel semi-fabricated products from World Steel Association (2011). Unless transportation data have been specified, raw materials are modeled as transported to the manufacturing plant(s) via diesel truck an assumed average distance of $805 \mathrm{~km}$ (500 mi).

The manufacturing energy data (shown in the tables) were based on Ecoinvent (2018), and a China electric grid was used since manufacturing is assumed to be in China. Parts forming processes, such as metal rolling, injection molding, and extrusion are also included when appropriate for the plastic and metal parts. These data come from Ecoinvent (2018).

\subsection{Incandescent}

The bill of materials shown in Table 5-62 is based on a $100 \mathrm{~W}$ (soft white) incandescent bulb manufactured by General Electric. Other sources provided data available for $60 \mathrm{~W}$ incandescent bulbs $^{38}$, but Table B-12 of U.K. Department for Environment (2009) was used since its data is based on a detailed tear-down for a widely available product. Furthermore, when factoring in use phase energy, the BOM differences between $60 \mathrm{~W}$ and $100 \mathrm{~W}$ are negligible.

\section{Table 5-62 Incandescent Light Bulb Bill of Materials}

\begin{tabular}{lccl} 
Parts and Materials & \multicolumn{2}{c}{ Mass } & Notes \\
Ballast & $\mathbf{g}$ & $\mathbf{l b}$ & \\
Black glass insulation & 2.0 & $4.4 \mathrm{E}-3$ & Modeled as foam glass \\
Internal filler & 1.0 & $2.2 \mathrm{E}-3$ & Modeled as foam glass \\
Lamp & 2.0 & $4.4 \mathrm{E}-3$ & \\
Tin plate base & $2.0 \mathrm{E}-2$ & $4.4 \mathrm{E}-5$ & Modeled as chromium \\
Tungsten filament & 2.0 & $4.4 \mathrm{E}-3$ & Modeled as borosilicate glass tube \\
Internal glass & & & \\
Lens & 20.0 & $4.4 \mathrm{E}-2$ & Modeled as borosilicate glass tube \\
Globe (glass) & & & \\
Packaging & 4.0 & $8.8 \mathrm{E}-3$ & \\
Corrugated board & $\mathbf{3 1 . 0}$ & $\mathbf{6 . 8} \mathbf{~ E - 2}$ & \\
Total & &
\end{tabular}

The data sets used to model the incandescent system's parts and materials come from Ecoinvent (2018). U.K. Department for Environment (2009) used proxy data for materials not available in Ecoinvent (2018) or other publicly-available data or databases. These proxies were also used for BIRDS, and are indicated in the table notes. Raw materials are modeled as transported to the manufacturing plant via diesel truck an assumed average distance of $805 \mathrm{~km}$ (500 mi).

\footnotetext{
${ }^{37}$ International Copper Association (ICA) (2013).

${ }^{38}$ See, for example, Parsons (2006).
} 
No manufacturing data were available, but the parts forming (wire drawing, injection molding, extrusion, etc.) have been included with the upstream raw materials, accounting for at least some of the production energy.

\subsection{Compact Fluorescent Lamp (CFL)}

The bill of materials in Table 5-63 is based on a 23 W Philips Marathon Mini CFL. Other data were available for an $18 \mathrm{~W} \mathrm{CFL}^{39}$ which might have been preferable in terms of wattage identified in Table 5-55, but Tables B.10-B.11 of U.K. Department for Environment (2009) were used since the tear-down was quite detailed and for a widely available product. Furthermore, the difference in mass between the two sources was $3 \%$ to $5 \%$, with a similar bill of materials. Factoring in use phase energy, this difference becomes negligible.

\section{Table 5-63 CFL Bill of Materials}

\begin{tabular}{lccc} 
Parts and Materials & & Mass & \multicolumn{2}{l}{ Notes } \\
Ballast & $\mathbf{g}$ & $\mathbf{l b}$ & \\
PUR foam (rigid) & 3.0 & $6.6 \mathrm{E}-3$ & \\
Plastic base (Polyvinyl chloride, PVC) & 17.0 & $3.7 \mathrm{E}-2$ & \\
Printed wiring board & 4.0 & $8.8 \mathrm{E}-3$ & \\
Printed board assembly (PBA) - & & & \\
Polypropylene (PP) caps & 4.0 & $8.8 \mathrm{E}-3$ & \\
PBA- inductor (cast iron) & 7.0 & $1.5 \mathrm{E}-2$ & \\
PBA- inductor (copper) & 4.5 & $9.9 \mathrm{E}-3$ & \\
PBA - transistor (ABS copolymer) & 1.0 & $2.2 \mathrm{E}-3$ & \\
PBA - transistor (Aluminum) & 3.5 & $7.7 \mathrm{E}-3$ & \\
PBA - resistors, diodes, HV capacitor & 1.0 & $2.2 \mathrm{E}-3$ & Modeled as a logic type integrated circuit \\
PBA - torus magnet (cast iron) & 1.0 & $2.2 \mathrm{E}-3$ & \\
Lamp & & & \\
Electrode assembly - mercury gas & $4.0 \mathrm{E}-3$ & $8.8 \mathrm{E}-6$ & \\
Electrode assembly - chromium & 2.0 & $4.4 \mathrm{E}-3$ & \\
Copper pins & 2.0 & $4.4 \mathrm{E}-3$ & \\
Tin base plate & 5.0 & $1.1 \mathrm{E}-2$ & \\
Black glass insulation & 5.0 & $1.1 \mathrm{E}-2$ & Modeled as foam glass \\
Lens & & & \\
Glass tube & 34.0 & $7.5 \mathrm{E}-2$ & Modeled as borosilicate glass tube \\
Packaging & & & \\
Corrugated board & 4.0 & $8.8 \mathrm{E}-3$ & \\
Total & $\mathbf{9 8 . 0}$ & $\mathbf{2 . 2}$ E-1 & \\
\hline
\end{tabular}

NREL (2015) provided data for the polyvinyl chloride base, polypropylene caps, acrylontrilebutadiene-styrene (ABS) copolymer transistor, and aluminum (as an average mix of primary and secondary aluminum). Data for copper sheet and wire come from International Copper Association (ICA) (2012). The remaining data sets come from ecoinvent. U.K. Department for Environment (2009) used proxy data for materials and/or parts not available in ecoinvent or other publicly-available data or databases. These proxies were also used for BIRDS, and are indicated

${ }^{39}$ Ibid. 
in the table notes. Raw materials are modeled as transported to the manufacturing plant via diesel truck an assumed average distance of $805 \mathrm{~km}$ (500 mi).

No manufacturing data were available, but the parts forming (wire drawing, injection molding, extrusion, etc.) and manufacture of the individual electronics components have been included with the upstream raw materials, accounting for at least some of the production energy. For assembly, U.K. Department for Environment (2009) used data provided by ecoinvent - assembly of an LCD screen - which the authors determined to be a suitable proxy for CFL manufacturing. Per U.K. Department for Environment (2009), this surrogate was selected because an LCD screen is also a complex electrical product, involving circuits and components that are assembled, and the impacts were expressed on a per kg basis so the assembly of the lighting systems could be modeled based on their respective weights. This was a conservative assumption on the part of U.K. Department for Environment (2009) Four Elements tested this assumption with sensitivity analysis, which showed that it did not make a significant difference to the overall results. BIRDS used this same assumption, applying the assembly data to a $98.0 \mathrm{~g}(0.22 \mathrm{lb})$ system.

\subsection{Light-Emitting Diode (LED)}

The integrated LED system from Table B.2 and Table B.3 in U.K. Department for Environment (2009) was modeled, giving the LED system the opportunity to be retrofitted into existing lighting infrastructure. The LED inventory shown in Table 5-64 factored in 10 LED die. 
Table 5-64 LED Bill of Materials

\begin{tabular}{|c|c|c|c|}
\hline \multirow{2}{*}{$\begin{array}{l}\text { Parts and Material } \\
\text { Ballast }\end{array}$} & \multicolumn{2}{|c|}{ Mass } & \multirow[t]{2}{*}{ Notes } \\
\hline & g & lb & \\
\hline PUR foam (rigid) & 3.0 & $6.6 \mathrm{E}-3$ & \\
\hline Inductor (cast iron) & 6.0 & $1.3 \mathrm{E}-2$ & \\
\hline Inductor (Cu) & 4.0 & 8.8 E-3 & \\
\hline Zener Diodes & 0.1 & $2.2 \mathrm{E}-4$ & Modeled as an unspecified diode \\
\hline Capacitors (aluminum) & 5.0 & $1.1 \mathrm{E}-2$ & \\
\hline Resistor & 10.0 & $2.2 \mathrm{E}-2$ & \\
\hline Transistor & 3.0 & $6.6 \mathrm{E}-3$ & \\
\hline PCB (aluminum machined tooled block) & 100.0 & $2.2 \mathrm{E}-1$ & Modeled as aluminum \\
\hline Wiring $(\mathrm{Cu})$ & 2.0 & 4.4 E-3 & \\
\hline Solder paste (used for electronics) & 1.0 & $2.2 \mathrm{E}-3$ & \\
\hline Polypropylene (PP) housing & 35.0 & 7.7 E-2 & \\
\hline Integrated circuit & 1.0 & 2.2 E-3 & \\
\hline Polyethylene terephthalate (PET) film & 2.0 & 4.4 E-3 & \\
\hline \multicolumn{4}{|l|}{ Lamp } \\
\hline Black glass insulation & 6.0 & $1.3 \mathrm{E}-2$ & Modeled as foam glass \\
\hline Tinplate base & 3.0 & 6.6 E-3 & Modeled as a low-alloyed steel \\
\hline Copper pins & 0.1 & $2.2 \mathrm{E}-4$ & \\
\hline Base contacts $(\mathrm{Cu})$ & 0.4 & $8.8 \mathrm{E}-4$ & \\
\hline Base contacts (solder paste for electronics) & 0.2 & $4.4 \mathrm{E}-4$ & \\
\hline Plastic base (PVC) & 16.0 & $3.5 \mathrm{E}-2$ & \\
\hline Light emitting diodes (LED, 10 total) & 19.0 & 4.2 E-2 & \\
\hline \multicolumn{4}{|l|}{ Lens } \\
\hline Glass & 20.0 & 4.4 E-2 & Modeled as borosilicate glass tube \\
\hline Coating (aluminum) & 1.0 & $2.2 \mathrm{E}-3$ & \\
\hline \multicolumn{4}{|l|}{ Packaging } \\
\hline Corrugated board & 3.0 & 6.6 E-3 & \\
\hline Total & 240.8 & $5.3 \mathrm{E}-1$ & \\
\hline
\end{tabular}

NREL (2015) provided data for the PVC base, PP housing, PET film, and aluminum (as average production mix). Data for copper sheet and wire come from International Copper Association (ICA) (2012). The remaining data sets, including the production of the LEDs, come from ecoinvent. U.K. Department for Environment (2009) used proxy data for materials not available 
in ecoinvent or other publicly-available data or databases. These proxies were also used for BIRDS, and are indicated in the table notes. Raw materials are modeled as transported to the manufacturing plant via diesel truck an assumed average distance of 805 km (500 mi).

Data for LED die manufacturing comes from OSRAM Opto Semiconductors GmbH. (2009). Primary data were collected on the two main process stages to produce OSRAM's Golden Dragon Plus: the front end, where the $1 \mathrm{~mm}^{2}\left(1.6 \mathrm{E}-3 \mathrm{in}^{2}\right)$ semiconductor chip is fabricated, and back end, where the chip is contacted and packaged. See OSRAM Opto Semiconductors GmbH. (2009), pp.9-11, for more qualitative detail and schematics on the manufacture of the LED. Figure 6 in OSRAM Opto Semiconductors GmbH. (2009) presents the primary energy to produce one LED: approximately $0.41 \mathrm{kWh}$. Given that the other categories of data in the figure include materials production, an assumption was made that "common consumption" is energy at manufacturing, amounting to approximately $0.27 \mathrm{kWh}$ per LED, or $2.7 \mathrm{kWh}$ for $10 \mathrm{LEDs}$. No other manufacturing data could be gleaned from this study, but the ecoinvent data set on assembly of an LCD screen was used for assembly (see above discussion), and parts forming data were applied to other parts and materials listed in the table.

\subsubsection{Transportation to the Building Site through End-of-Life}

\subsection{Residential Lighting}

Numerous LCA studies mention China or Asia as being the main manufacturing location for incandescents. For their study, Energy Efficiency and Renewable Energy (EERE) (2012), p.32, assumed that an incandescent lamp is either manufactured in the northeastern U.S. or Shanghai, China, while also acknowledging that production occurs all over the world. BIRDS assumed 25 $\%$ of production of incandescents to be produced in the northeast U.S., and $75 \%$ in Shanghai, China.

BIRDS modeled CFLs as manufactured in China (Parsons 2006, Ramroth 2008, U.K. Department for Environment 2009, Energy Efficiency and Renewable Energy (EERE) 2012).

Energy Efficiency and Renewable Energy (EERE) (2012) stated that LED lighting market is highly fragmented, with several firms focusing on a specific part within the LED supply chain (this was seen with OSRAM Opto Semiconductors GmbH. (2009)). BIRDS adopted EERE's approach to simplifying the sourcing logistics as follows: "the complete LED package is produced in Taiwan and then is assembled into the finished LED lamp product in Taiwan or the United States. In the second scenario, LED packages are produced in Taiwan and then shipped to the southeast region of the U.S. where they are assembled into complete LED lamp products” (Energy Efficiency and Renewable Energy (EERE) 2012).

For Asia manufacturing, transportation includes the ocean freighter transport to a port in California, plus heavy duty diesel truck an assumed 2414 km (1500 mi), an average distance traveled from California to other parts of the United States. For U.S. manufacturing, transport is modeled an assumed average of $2414 \mathrm{~km}$ (1500 mi) by heavy-duty diesel fuel-powered truck. Intermediate transportation (i.e., from LED production to lamp assembly) is also included. 
For every residential light bulb purchased and needing to be replaced, customer driving to and from the retail store has been included.

How these residential lighting systems affect operating energy during the home's use phase is addressed in other sections of this report.

At end-of-life, each of the residential lighting alternatives was modeled as $20 \%$ recycling (and material recovery), and $80 \%$ landfill. A distance of $48 \mathrm{~km}(30 \mathrm{mi})$ traveled via a heavy-duty diesel truck to the landfill or recycler has been assumed for waste and recycling transport.

\subsection{Commercial Lighting}

The linear fluorescent lighting systems modeled for BEES are assumed to be manufactured in China. Transportation includes the ocean freighter transport to a port in California, plus heavy-duty diesel truck an assumed $2414 \mathrm{~km}$ (1500 mi), an average distance traveled from California to other parts of the United States. Intermediate transportation (i.e., from fluorescent bulb production to lamp assembly) is also included.

How these lighting systems affect operating energy during the buildings' use phase is addressed in other sections of this report.

At end-of-life, the used fluorescent bulbs are dismantled and treated. Operations include dry dismantling, cutting, blowing, crushing, and air exhaust cleaning. Data come from ecoinvent. Ballasts at end of life are modeled using the ecoinvent dataset for treatment of electronic control equipment, which includes dismantling the electronics components (printed wiring boards, etc.) and sending the other components to recyclers and incineration. The fixture at end of life is modeled as sent to treatment of electronics scrap and recycling. 


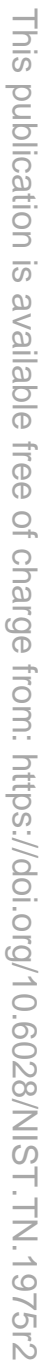




\section{Economic Performance Measurement}

When a decision maker wants a dollar measure of cost-effectiveness, and cash flows are the primarily costs, the most appropriate method for measuring the economic performance of a building is life-cycle costing (LCC; (Fuller and Petersen 1996, ASTM 2015). BIRDS follows the ASTM International standard method for LCC of building-related investments (ASTM 2015), which involves calculating a present value of a cost by discounting its future value into Year 0 , 2015 for the current version of BIRDS, dollars based on the year the cost occurs and the assumed discount rate. The formulas and discount factors used to calculate the present values will vary depending on the type of cost. The different cost types and related formulas, discount factors, data sources, and adjustment factors are described below. ${ }^{40}$

\subsection{First Cost}

The initial cost component of building LCCs are first costs. The first costs of a building are the total costs of constructing a building in a particular city. First costs include costs of labor, materials, equipment, overhead, and profit.

\subsubsection{Approach}

The construction costs for a prototype building are estimated by summing the costs of the baseline building ( $C_{\text {NatAvg }}$ ) and the changes in costs required to meet the prototype building design $\left(\Delta C_{x}\right)$, adjusted for location-related cost variation as well as contractor and architectural profits. Both the baseline building costs and component cost estimates are based on national average construction cost data. The RSMeans Online Square Foot Cost Estimator (SFCE) is used to estimate average baseline building costs, which are based on the basic characteristics and features of the prototype buildings described in Chapter 3 (RSMeans 2016). Since costs are already in 2016 dollars, there is no need to adjust for inflation. A screenshot of the SFCE is shown in Figure 6-1.

\footnotetext{
${ }^{40}$ See Kneifel (2012) for additional details on the cost data used in the BIRDS Database
} 


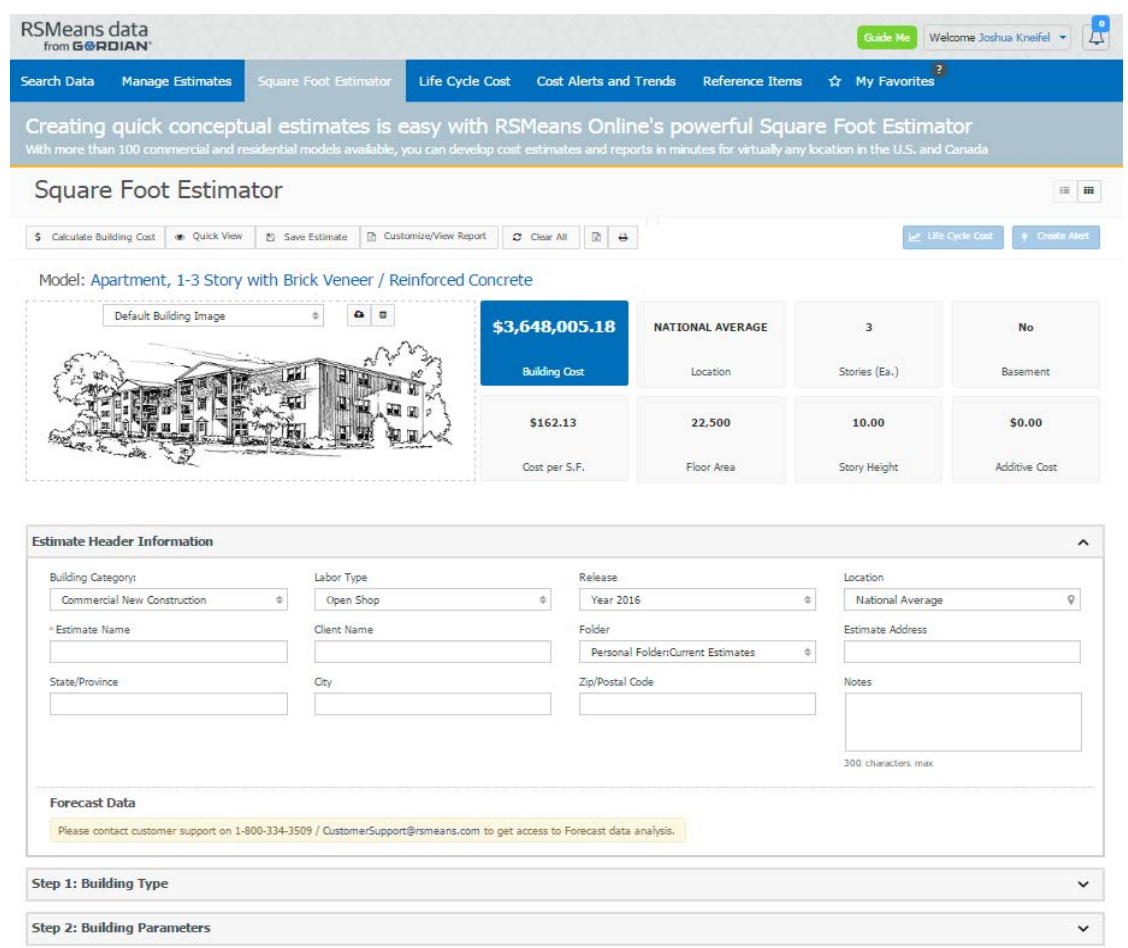

Figure 6-1 RSMeans Online Square Foot Cost Estimator (SFCE)

Adjustments in national average construction costs are done using the 2016 RSMeans CostWorks City Indexes to control for local material and labor price variations in the 228 locations for which the energy simulations are run. The "weighted average" city construction cost index $\left(I_{\text {WAvg }}\right)$ is used to adjust the costs for the baseline prototypical building while "component" city indexes $\left(I_{x}\right)$ are used to adjust the costs for the change in component designs. The formula below shows the indexed construction cost $\left(C_{\text {Index }}\right)$ calculation.

$$
\begin{gathered}
C_{\text {Index }}=\left(C_{N a t A v g} * I_{W A v g}\right)+\left(\Delta C_{H V A C} * I_{H}\right)+\left(\Delta C_{\text {Wall }}+\Delta C_{\text {Roof }}+\Delta C_{\text {Foundation }}\right) * I_{T}+\left(\Delta C_{\text {Light }}\right) \\
* I_{E}+\left(\Delta C_{\text {Window }}\right) * I_{O}
\end{gathered}
$$

Where $C_{\text {Index }}=$ Indexed construction costs

$C_{\text {NatAvg }}=$ National average construction costs

$\Delta C_{H V A C}=$ Change in HVAC system costs

$\Delta C_{\text {Wall }}=$ Change in wall insulation costs

$\Delta C_{\text {Roof }}=$ Change in roof insulation costs

$\Delta C_{\text {Foundation }}=$ Change in foundation costs

$\Delta C_{\text {Light }}=$ Change in lighting costs

$\Delta C_{\text {Window }}=$ Change in window costs

$I_{H}=$ "Fire Suppression, Plumbing, \& HVAC" cost index

$I_{T}=$ "Thermal and Moisture Protection" cost index

$I_{E}=$ "Electrical, Communications, \& Utilities" cost index

$I_{O}=$ "Openings" cost index 
Once the indexed construction costs of the building are calculated, it is necessary to adjust for the contractor and architect profits by multiplying the costs by the contractor "mark-up" rate $\left(I_{M}\right)$, assumed to be $25 \%$, and then the architectural fees rate $\left(I_{A}\right)$, assumed to be $7 \%$, as shown in the following equation.

$$
C_{\text {First }}=\left(C_{\text {Indexed }} *\left(1+I_{M}\right)\right) *\left(1+I_{A}\right)
$$

The mark-up rates are based on the default values used by the RS Means SFCE. The marked-up, indexed construction costs are the first costs of constructing the prototype building in the city $\left(C_{\text {First }}\right)$.

\subsubsection{Data}

Building construction costs are obtained from the RSMeans online cost databases. The costs of a prototypical building are estimated by the RSMeans Online's SFCE to obtain the default costs for each BIRDS building type for each component. The RSMeans default building serves as the baseline and is used to create a building that is compliant with each of the five energy efficiency alternatives: ASHRAE 90.1-2004, ASHRAE 90.1-2007, ASHRAE 90.1-2010, ASHRAE 90.1-2013, and ASHRAE 90.1-2016. The default buildings are adapted to match the fifteen prototype building designs using the RSMeans Online cost database.

\subsubsection{Wall, Roof, and Foundation Insulation}

RSMeans unit cost data is available for different types and R-values of insulation on a per unit of area basis for use in wall, roof, and foundation assemblies. The insulation requirements for a given building envelope assembly varies by edition of ASHRAE 90.1 and the climate zone in which the building is located. For each building type-location-standard edition combination, the cost of meeting the insulation requirement for an assembly is compared to the cost of the baseline insulation levels to estimate the change in construction costs for each assembly to meet the standard edition requirements.

Each baseline prototypical building has an assumed wall assembly that is categorized into four types with the assumed baseline wall insulation levels as shown in Table 6-1. The insulation requirements for a given building type-location-standard edition combination is matched to the appropriate insulation level shown in Table 5-7, and its associated cost per unit of area installed. The difference between the cost per unit of area of the required insulation level and the baseline insulation level multiplied by the total exterior wall area is the change (delta) in cost to meet the standard edition requirement for that building type in that location $\left(\Delta C_{\text {Wall }}\right)$.

\section{Table 6-1 Baseline Wall Insulation}

\begin{tabular}{lc} 
Adjustment & Baseline Insulation \\
\hline Mass-CME & R-5.7 c.i. \\
\hline Metal Building & R-0+R-9.8 c.i. \\
\hline Steel-Framed & R-13 \\
\hline Wood-Framed & R-13+R-3.8 c.i. \\
\hline Note: R-1 = R SI-0.18 \\
\hline
\end{tabular}


The delta costs for roof insulation ( $\Delta C_{\text {Roof }}$ ) are calculated using the same method with data for the three roof assemblies from Table 5-8 and the baseline roof insulation level from Table 6-2 that meets the standard edition requirements for a building type located in a specific location.

\section{Table 6-2 Baseline Roof Insulation}

\begin{tabular}{lc} 
Adjustment & Baseline Insulation \\
\hline Attic and Other & R-30 \\
\hline Metal Building & R-10+R-19 FC \\
\hline IEAD & R-15.0 c.i. \\
\hline Note: R-1 = R RI-0.18 & \\
\hline
\end{tabular}

The delta costs for foundation insulation ( $\Delta C_{\text {Foundation }}$ ) are calculated using the same method with data for the three roof assemblies from Table 5-10 and the baseline foundation insulation level from Table 6-3 that meets the standard edition requirements for a building type located in a specific location.

\section{Table 6-3 Baseline Foundation Insulation}

\begin{tabular}{lcc} 
Adjustment & $\begin{array}{c}\text { Baseline Floor } \\
\text { Insulation and Depth }\end{array}$ & $\begin{array}{c}\text { Baseline Wall } \\
\text { Insulation }\end{array}$ \\
\hline Basement & $\mathrm{NR}$ & $\mathrm{NR}$ \\
\hline Slab on Grade & $\mathrm{R}-0$ & $\mathrm{~N} / \mathrm{A}$ \\
\hline Note: $\mathrm{R}-1=\mathrm{R}_{\mathrm{SI}}-0.18 ; \mathrm{NR}=$ No Requirement; $\mathrm{N} / \mathrm{A}=$ Not Applicable \\
\hline
\end{tabular}

\subsubsection{Interior Lighting}

All baseline prototypical buildings defined by RSMeans, except for the apartment buildings, utilize T8, energy saver 32-watt interior fluorescent light fixtures (recess mounted in the ceiling). Changes in the total number of fixtures, or total wattage, is based on comparisons between RSMeans baseline buildings and alternative prototypical building models designed per editions of 90.1, and found by taking the difference in total installed (difference in LPDs multiplied by total square footage) wattage between the two designs. Changes in lighting costs $\left(\Delta C_{\text {Light }}\right)$ due to changes in total installed wattage can then be found by multiplying the difference in wattage by the RSMeans average cost per watt for the T8 fluorescent bulb. ${ }^{41}$

The prototypical mid-rise and high-rise apartment buildings in RSMeans both include recess mounted incandescent lighting fixtures with a LPD of 1.0. For each edition of ASHRAE 90.1 considered in BIRDS, the LPDs for both apartment buildings are less than 1.0 - implying that there will be a reduction in the total number of installed fixtures in the building for each edition of the standard. We also assume that this is done in conjunction with the efficiency of the overall lighting system increasing. For this to occur, the incandescent lighting must first be replaced with compact fluorescent lighting (CFL). The number of fixtures is then reduced to meet the remainder of the required reduction in watts per unit of floor area. The $\Delta C_{\text {Light }}$

\footnotetext{
${ }^{41} \$ 3.16$ is the average cost per watt and is based on multiple price points for the T8 lighting fixture in RSMeans.
} 
component between the baseline prototype building and an ASHRAE 90.1 alternative is found by multiplying the total wattage of incandescent lighting replaced by CFLs by the change in lighting cost per watt equivalent. ${ }^{42}$ Increasing the efficiency of the lighting system increases construction costs or first costs.

\subsubsection{Windows}

Window cost estimates use a similar approach as the window LCIA estimates. The window performance specifications from the simulation model are mapped to the most comparable window type with the appropriate window characteristics to meet those specifications based on data from the Efficient Windows Collaborative (www.efficientwindows.org). Each simulation is matched to the window type and one of these potential set of window characteristics:

- Standard Glass Window

- Standard Glass Window with Tint

- Insulated Glass Window

- Insulated Glass Window with Tint

- Insulated Glass Window with Thermal Break Frame

- Insulated Glass Window with Thermal Break Frame and Tint

- Insulated Glass Window with Thermal Break Frame and Low-Emissivity (Low-E) Coating

- Insulated Glass Window with Thermal Break Frame and Tint and Low-E Coating

The costs for the windows in the RS Means baseline cost estimate are adjusted based on the necessary changes to meet the window performance specifications with the mark-up factors listed in Table 6-4, which include mark-ups for glazing, tints, coatings, framing, and operability. All these mark-ups are based on RS Means cost data, either as direct suggested mark-ups (e.g. low-E coating) or through comparisons of cost estimates for similar windows with different characteristics (e.g. standard versus insulated glazing). In the case where the cost data suggests a mark-up for a portion of the window (glazing only or frame only), the mark-up is converted into a mark-up for the entire window based on the fraction of the total window cost associated with that portion of the window.

${ }^{42}$ The approach used for the two apartment buildings is based on Belzer, Cort et al. (2005) and Halverson, Gowri et al. (2006). 
Table 6-4 Window Cost Adjustments

\begin{tabular}{lcl} 
Adjustment & Mark-Up & \multicolumn{1}{c}{ Details } \\
\hline Tint & $12 \%$ & $\begin{array}{l}\text { Suggested glazing cost mark-up value for bronze finish } \\
\text { by RS Means, adjusted to fraction of total window cost }\end{array}$ \\
\hline Low E coating & $15 \%$ & $\begin{array}{l}\text { Suggested value by RS Means, adjusted to fraction of } \\
\text { total window cost }\end{array}$ \\
\hline Thermal Break & $12 \%$ & $\begin{array}{l}\text { Typical difference between standard and thermal break } \\
\text { frame, adjusted to fraction of total window cost }\end{array}$ \\
\hline Insulated Glazing & $12 \%$ & $\begin{array}{l}\text { difference in standard versus insulated windows for } 5 x 3 \\
\text { window }\end{array}$ \\
\hline Fixed to Operable & $50 \%$ & $\begin{array}{l}\text { Comparable change in same sized windows fixed vs } \\
\text { operable }\end{array}$ \\
\hline
\end{tabular}

Based on these specifications and the incremental cost mark-ups from Table 5-14, the total markup for a given building simulation's window specifications can be estimated. Mark-ups are additive in nature. For example, if the baseline window in the RS Means estimate is an insulated aluminum operable window, but the simulation performance specifications imply an operable, insulated window with low-E coating and thermal break frame then the mark-up would be $15 \%$ $+12 \%=27 \%$ higher than the total baseline window costs.

It is acknowledged that there are multiple window assembly options (combination of frame material, glass in-fill, and operability) for any building type in any climate zone; multiple window assembly options can be nearly identical in performance. The window assembly types presented in these tables are only one of many options available. As such, the window assembly combinations presented in this documentation are not endorsed or preferred over any other type of window assembly for the respective buildings in which they are used in BIRDS.

It should also be noted that WWRs vary greatly for many different reasons. Those used in BIRDS are based solely on the DOE Reference Building Simulation Models, and may not be representative of WWRs for actual buildings of similar types.

\subsubsection{HVAC}

Estimating initial HVAC system costs for each prototype building is completed using a series of heating and cooling equipment cost functions based on RSMeans cost data, and the resulting HVAC system component capacities from each of 13680 building simulations in the new commercial database. First, the HVAC equipment in each prototype building model is mapped to the most comparable equipment in the RSMeans database. Second, a best fit functional form is then derived for each piece of equipment using the RSMeans cost data, where equipment costs are a function of equipment capacity. ${ }^{43}$ This process is repeated for every HVAC system component option included in the prototype buildings. Lastly, total HVAC system costs for a given simulation are calculated by using the auto-sized capacities of each HVAC system

\footnotetext{
${ }^{43}$ Estimated HVAC equipment cost formulas are independent of equipment efficiency given the lack of available data in RSMeans for equipment costs at varying levels of efficiency. Thus, functional forms were derived based on RSMeans data we believed was best representative of equipment at standard or typical efficiency - leading to $\Delta C_{H V A C}$ estimates that may be slightly under- or overstated. In either case, we assume the delta to be negligible.
} 
component and the appropriate cost functions to generate a single component cost. The total cost is then found by summing up the individual costs of all HVAC system components. Although RSMeans provides majority of the HVAC equipment cost data, some external sources were used to supplement this data. ${ }^{44}$

\subsection{Future Costs}

The second LCC component for a building are future costs. Future costs account for all of the costs associated with operation of a building, as well as the costs of properly maintaining, repairing, and replacing components of the building.

\subsubsection{Approach}

Building maintenance, repair, and replacement (MRR) costs are discounted to equivalent present value dollars using the Single Present Value (SPV) factors for future non-fuel costs reported in Lavappa and Kneifel (2016). These factors are calculated using the DOE Federal Energy Management Program (FEMP) 2016 real discount rate for federal energy conservation projects (3\%), and a value that more closely represents the long-run real return on equities (8\%). ${ }^{45}$ Table 6-5 reports the SPV factors for the $3 \%$ and $8 \%$ discount rates included in the new BIRDS commercial database. The MRR costs in each year $\left(C_{M R R, i}\right)$ are discounted to year 2016 dollars by multiplying the MRR costs in year $i$, by the discounting factor for year $i$. The total present value MRR costs $\left(C_{M R R}\right)$ are then calculated by summing up all the MRR costs in each year.

\section{Table 6-5 2016 SPV Discount Factors for Future Non-Fuel Costs, 8 \% and 3 \% Real Discount Rate}

\begin{tabular}{ccc} 
Yrs & \multicolumn{2}{c}{ SPV Factor } \\
& $8 \%$ & $3 \%$ \\
\hline 1 & 0.92 & 0.97 \\
\hline 2 & 0.85 & 0.94 \\
\hline 3 & 0.79 & 0.91 \\
\hline 4 & 0.73 & 0.88 \\
\hline 5 & 0.68 & 0.86 \\
\hline 6 & 0.63 & 0.83 \\
\hline 7 & 0.58 & 0.81 \\
\hline 8 & 0.54 & 0.78 \\
\hline 9 & 0.50 & 0.76 \\
\hline 10 & 0.46 & 0.74 \\
\hline
\end{tabular}

\begin{tabular}{ccc} 
Yrs & \multicolumn{2}{c}{ SPV Factor } \\
& $8 \%$ & $3 \%$ \\
\hline 11 & 0.42 & 0.72 \\
\hline 12 & 0.39 & 0.70 \\
\hline 13 & 0.36 & 0.68 \\
\hline 14 & 0.34 & 0.66 \\
\hline 15 & 0.31 & 0.64 \\
\hline 16 & 0.29 & 0.62 \\
\hline 17 & 0.27 & 0.60 \\
\hline 18 & 0.25 & 0.58 \\
\hline 19 & 0.23 & 0.57 \\
\hline 20 & 0.21 & 0.55 \\
\hline
\end{tabular}

\begin{tabular}{ccc} 
Yrs & \multicolumn{2}{c}{ SPV Factor } \\
& $8 \%$ & $3 \%$ \\
\hline 21 & 0.19 & 0.53 \\
\hline 22 & 0.18 & 0.52 \\
\hline 23 & 0.17 & 0.50 \\
\hline 24 & 0.15 & 0.49 \\
\hline 25 & 0.14 & 0.47 \\
\hline 26 & 0.13 & 0.46 \\
\hline 27 & 0.12 & 0.45 \\
\hline 28 & 0.11 & 0.43 \\
\hline 29 & 0.10 & 0.42 \\
\hline 30 & 0.09 & 0.41 \\
\hline
\end{tabular}

\begin{tabular}{ccc} 
Yrs & \multicolumn{2}{c}{ SPV Factor } \\
& $8 \%$ & $3 \%$ \\
\hline 31 & 0.09 & 0.40 \\
\hline 32 & 0.08 & 0.38 \\
\hline 33 & 0.07 & 0.37 \\
\hline 34 & 0.07 & 0.36 \\
\hline 35 & 0.06 & 0.35 \\
\hline 36 & 0.06 & 0.34 \\
\hline 37 & 0.05 & 0.33 \\
\hline 38 & 0.05 & 0.32 \\
\hline 39 & 0.05 & 0.31 \\
\hline 40 & 0.04 & 0.30 \\
\hline
\end{tabular}

The electricity and natural gas use predicted by the building's energy simulation is used as the annual energy use of the building for each year of the selected study period. Electricity and natural gas prices are assumed to change over time according U.S. Energy Information Administration forecasts from 2017 to 2046. These forecasts are embodied in the FEMP

\footnotetext{
${ }^{44}$ Additional HVAC cost data/functional forms for the heating and cooling components of a typical residential split system was provided by Dr. Yeonjin Bae of Purdue University (Bae 2016). Cost formulas were functional on both equipment capacity and efficiency.

${ }^{45}$ Note that an $8.0 \%$ real discount rate is a relatively high rate relative to the long-run real-return on equities of approximately $6.6 \%$ to $6.7 \%$ (Siegel 2014).
} 
Modified Uniform Present Value Discount Factors for energy price estimates (UPV*) reported in Lavappa and Kneifel (2016). Multiplying the annual electricity costs and natural gas costs by the associated UPV* value for the study period of interest estimates the present value total electricity $\operatorname{cost}\left(C_{\text {Elect }}\right)$ and natural gas costs $\left(C_{\text {Gas }}\right)$. The discount factors vary by census region, end use, and fuel type.

Total present value future costs ( $C_{\text {Future }}$ ) is the sum of present value location-indexed MRR costs and present value energy costs, as shown in Equation 6.1:

$$
C_{\text {Future }}=C_{M R R}+C_{\text {Elect }}+C_{\text {Gas }}
$$

\subsubsection{Data - Maintenance, Repair, and Replacement}

Component and building lifetimes and component repair requirements are based on data from the Whitestone Research CostLab Tool (CBRE 2016). This online reference tool assists facility professionals in estimating facility operating and MRR costs. Building service lifetimes are assumed constant across climate zones: apartment buildings last for 65 years; hotels, schools, office buildings, retail stores, and restaurants for 41 years. ${ }^{46}$ Building component MRR rates are also from Whitestone (2016). Insulation and windows are assumed to have a lifespan equal to the life of the building and have no maintenance requirements. Insulation is assumed to have no repair costs. The assumed annual window repair costs vary depending on the building type and window specifications. The heating and cooling units have different lifespans and repair frequencies based on system type, ranging from 1 year to 14 years for repairs and 14 years to 28 years for replacements.

The Whitestone Research CostLab Tool was also used to collect maintenance, repair and replacement costs. The total maintenance and repair costs per square foot of conditioned floor area (minus the maintenance and repair costs for the HVAC system, lighting system, and windows) represent the baseline maintenance and repair costs per unit of floor area, which occur for a building type regardless of the energy efficiency measures incorporated into the design. CostLab reports average maintenance and repair costs per unit of floor area by building component for each year of service life for each building type. The building types in Whitestone do not match exactly to the 15 DOE prototype buildings selected for this study - therefore, each prototype building is matched to Whitestone buildings with the most comparable profiles.

CostLab also served as the source of maintenance and repair costs for the individual components for which the MRR costs change across alternative building designs, which in this analysis are the HVAC system, lighting system, and windows. Lighting systems are assumed to be replaced every 20 years, without any necessary routine maintenance or repair. The HVAC system sizes vary based on the thermal performance of the building design, which results in varying MRR costs because smaller systems are relatively cheaper to maintain, repair and replace. To maintain consistency between the first costs and the replacement costs data for the HVAC system, lighting system, and windows, the building component cost data collected using the RS Means

\footnotetext{
${ }^{46}$ Building service life is based on data from Whitestone 2010.
} 
CostWorks database serves as estimates for both the first costs and replacement costs. All future MRR costs are discounted to equivalent value dollars using the assumed discount rate.

Annual energy costs are estimated by multiplying annual electricity and natural gas use predicted by the building's energy simulation by the average state retail commercial electricity and natural gas prices, respectively. Average state commercial electricity and natural gas prices for 2016 are collected from the Energy Information Administration (EIA) Electric Power Annual State Data Tables and Natural Gas Navigator, respectively.

\subsection{Residual Value}

The third and final component of LCC is the residual value. A building's residual value is its value remaining at the end of the study period. In life-cycle costing it is treated as a negative cost item. In BIRDS, it is estimated in two major parts based on the approach defined in Fuller and Petersen (1996): the building excluding components that get replaced (e.g., HVAC, windows, and lighting system), and the building components that get replaced (e.g., HVAC, windows, and lighting). The building's residual value (Equation 6.2) is calculated as the building's locationindexed first cost (excluding HVAC, windows, and lighting costs) multiplied by one minus the ratio of the study period to the service life of the building, discounted from the end of the study period using the SPV factor for the last year of the study period (T).

$$
R V=\text { First Cost } \cdot\left(1-\frac{\text { Study Period }}{\text { Service Life }}\right) \cdot S P V_{T}
$$

For example, if a building has first costs (excluding HVAC and lighting) of \$1 million, a 41-year service life, and the study period length is 10 years, the residual value of the building in year 10 (excluding HVAC, windows, and lighting) is:

$$
\$ 1000000 \cdot\left(1-\frac{10}{41}\right) \cdot 0.744=\$ 562537 .
$$

Because they may be replaced during the study period, residual values for the HVAC system, windows, and lighting systems are computed separately. The remaining "life" of the HVAC equipment is determined by taking its service life minus the number of years since it last installation (as of the end of the study period), whether it occurred during building construction or replacement. The ratio of remaining life to service life is multiplied by the location-indexed installed cost of the system and discounted from the end of the study period. For example, assume an HVAC system's installed costs are \$100 000 with a service life in the selected location of 8 years, and a 10-year study period length. After one replacement, the system is 2 years old at the end of the study period, leaving 6 years remaining in its service life. The residual value in year 10 is:

$$
\$ 100000 \cdot \frac{6}{8} \cdot 0.744=\$ 55800 .
$$

The residual values for the windows and lighting systems are computed in a similar manner. The total residual value of the building and its HVAC system, windows, lighting systems, multiplied 
by the SPV factor for the number of years in the study period, estimates the present value residual value $\left(C_{\text {Residual }}\right)$.

\subsection{Life-Cycle Cost Analysis}

The total life-cycle cost of a prototype building $\left(C_{L C C}\right)$ is the sum of the present values of first cost and future costs minus the residual value as shown in Equation 6.5:

$$
C_{\text {LCC }}=C_{\text {First }}+C_{\text {Future }}-C_{\text {Residual }} \text {. }
$$

LCC analysis of buildings typically compares the LCC for a "base case" building design to the costs for alternative, more energy efficient building design(s) to determine if future operational savings justify higher initial investments. For BIRDS, total life-cycle costs are calculated as described above for all building design options for all study periods. The user of the tool has the option to select any of the building designs as the base case, and compare it to any of the alternative designs. For an investor comparing mutually exclusive design alternatives, the same study period must be used for all alternatives. For those interested in the sensitivity of LCC results to the assumed study period length, BIRDS allows the study period length for a given building design to vary.

Two metrics are used to analyze changes in life-cycle costs: net LCC savings and net LCC savings as a percentage of base case LCC. Net LCC savings (NS) is the difference between the base case LCC $\left(C_{\text {Base }}\right)$ and alternative design LCC $\left(C_{A l t}\right)$ as shown in Equation 6.6:

$$
N S=C_{\text {Base }}-C_{\text {Alt }} \text {. }
$$

Net LCC savings as a percentage of base case LCC (PNS) is the net LCC savings divided by the base case LCC. This metric, shown in the equation below, allows for comparisons across building types that vary significantly in terms of floor area.

$$
P N S=\frac{N S}{C_{\text {Base }}} * 100 \text {. }
$$




\section{Software Development and Design}

The BIRDS software tool is a data-driven web application that enables access to one of three NIST-developed buildings databases: (1) energy standard/code-based commercial, (2) energy standard/code-based residential, and (3) incremental energy efficiency residential. The recently updated commercial database discussed in this report includes more than a million records. It is based on 17100 energy simulations as described in previous sections of this report. Comparisons of life-cycle costs, operating energy consumption, or environmental impacts for different building designs can be evaluated using the data visualization features in the application. Technologies were selected for this project based on their utility in developing this comprehensive system. The sections below summarize each of the technologies used and their role(s) in the development of the BIRDS software tool.

\subsection{Database Creation and Management}

Both Python and the Microsoft Sql Server relational database management system are used to develop and manage each of the databases in BIRDS. Python, a widely used, general purpose programming language is used to create programs for generating the final data set, which in the case of the commercial database contains more than 1 million records. Python scripts have been developed to generate LCC data, operating energy data, and environmental impact data from location specific building simulation data. Once the data sets have been generated, the Microsoft Sql Server relational database management system is then used to store each database. Creating efficient indexes is important for reducing fetch times for records so the application will display data quickly.

\subsection{Application}

The application has been written using the C\# programming language within the .NET development framework in combination with several web development tools to create the web interface and the associated databases. Each of these tools will be summarized in the remainder of this section.

\subsubsection{Software Programming Language - C\#}

$\mathrm{C \#}$ is an object-oriented programming language developed by Microsoft. It is based on the C++ programming language, has many similarities with Java, and was developed to work with the .Net framework. C\# is used primarily for developing the server side code of BIRDS v4.0, including modules to process results data for visualization and data retrieval.

\subsubsection{Software Framework - .NET}

The .Net Framework is a Microsoft developed framework, which contains the common language runtime, in addition to several common class libraries. The common language runtime can be thought of as the foundation of the framework that manages processes at execution time. BIRDS targets the .Net Framework version 4.5. 


\subsubsection{Web Development Technologies - HTML, CSS, JavaScript, JQuery, jqChart}

Several web technologies were used in the creation of the user interface. Hypertext Markup Language (HTML) is the primary language used for displaying web content. Cascading Style Sheet (CSS) is the definition file used by web pages for formatting. JavaScript is a light-weight scripting language used to programmatically manipulate the input, output or display of a web page. JQuery is a JavaScript library that facilitates Document Object Model (DOM) manipulation and simplifies partial web page data refreshing through asynchronous JavaScript and XML (AJAX) requests. jqChart is a html5 charting library used to render charts based on the data for specific comparisons in BIRDS v4.0.

\subsubsection{Application Design - Visual Studio}

The BIRDS application is developed using Visual Studio's MVC 5 project template with the "database first method." Model, view, and controller functions are all placed in different code files to keep each entity separate. The general flow of the application can be seen in Figure 6-1. A user makes a request through the browser. The controller gets the request and passes the request parameters to the model, which retrieves necessary data from the database. The model passes back the data, which is merged with the view and then passed back to the browser by the controller.
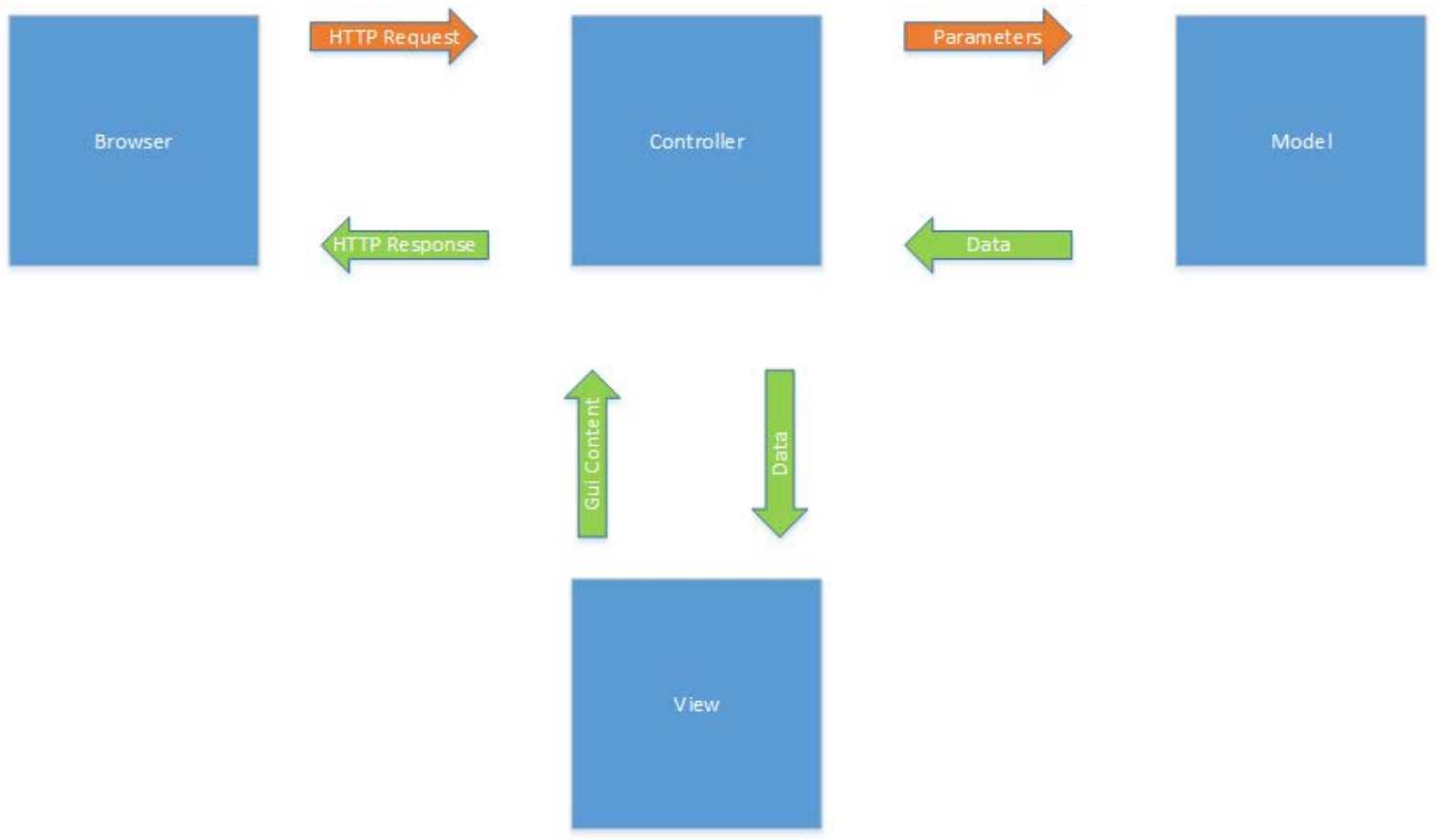

\section{Figure 7-1 Application Information Flow}

In developing the BIRDS low-energy application, the database first method is used because the BIRDS commercial database had been developed prior to development of the user interface module. The model containing fourteen tables was created based on the database. Controller methods were then developed to retrieve data based on specific parameters from the model, 
which was then combined with the view and passed back to the browser to be displayed to the user. Comparison results are displayed by the chart module, which contains functions for ordering, formatting, and displaying data for different chart types. The capability to download the data in a .csv file is included so that data can be analyzed by the user according to their preferences. 


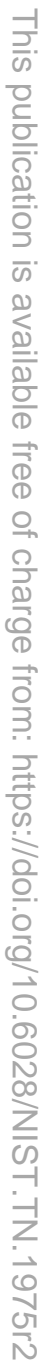




\section{Limitations and Future Research}

The BIRDS tool is currently limited in scope, and the depth of the tool would be greatly strengthened by including additional building prototypes, more location-specific data, uncertainty analysis, metrics, and flexibility.

The 15 prototypical commercial buildings analyzed for the purposes of extending the BIRDS database are likely not representative of the entire building stock for each building type. For example, all high-rise buildings are not $100 \%$ glazed. For this reason, the results should be considered as general magnitudes for making reasonable comparisons instead of hard numbers. The existing PNNL prototype buildings are representative of $80 \%$ of the U.S. commercial building stock. As additional prototype buildings are developed representing increasing shares of domestic building stock, future research should include these additional prototypes in the BIRDS. The state average energy cost rates and energy-related carbon emissions rates do not control for local variation in energy tariffs or electricity fuel mixes. By using utility-level energy cost and emissions rate data, the accuracy of the estimates in BIRDS could be improved.

Uncertainty analysis is needed for at least two elements of the analysis. First, sensitivity analysis on the assumed discount rate (3\% or $8 \%$ ) is needed to determine the robustness of the cost results. Second, the BIRDS environmental impact scores do not incorporate uncertainty analysis as required by international standards (ISO, 2006). While incorporating uncertainty analysis is problematic due to a lack of underlying uncertainty data, this omission should be brought into the interpretation of the BIRDS results.

All the BIRDS databases ignore the impacts that plug and process loads have on potential reductions in operating energy use. Buildings with greater plug and process loads will realize smaller percentage changes in energy use because the energy efficiency measures considered in this study focus on the building envelope and HVAC equipment, holding constant the energy use from other equipment used in the building. As building energy efficiency improves, the plug and process loads become a larger fraction of the overall energy load. Future research should consider the impact the assumed plug and process loads have on the overall energy savings realized by energy efficiency improvements to buildings.

Properly interpreting the BIRDS environmental performance results requires placing them in perspective. The environmental impact scores assess the life-cycle impacts of operating energy use based on inventories of localized energy simulation results and regional electricity grids. All other elements of the scores-including a building's use of materials and its water consumption over the study period - are based on U.S. average life-cycle inventory data for prototypical buildings. The baseline data for these buildings represent status quo building technologies as of 2002, the year of the latest available input-output data from the U.S. Bureau of Economic Analysis at the time of the I-O LCIA data development. To account for evolution in status quo technologies over time, future versions of BIRDS should incorporate newer releases of these data as they become available.

The BIRDS LCAs use selected inventory flows converted to selected local, regional, and global environmental impacts to assess environmental performance. Those inventory flows which 
currently do not have scientifically proven or quantifiable impacts on the environment are excluded, such as mineral extraction and wood harvesting, which are qualitatively thought to lead to loss of habitat and an accompanying loss of biodiversity. If the BIRDS user has important knowledge about these issues, it should be brought into the interpretation of the BIRDS results.

The Environmental Problems approach that BIRDS uses for impact assessment does not offer the same degree of relevance for all environmental impacts. For global and regional effects (e.g., global warming and acidification) the method may result in an accurate description of the potential impact. For impacts dependent upon local conditions (e.g., smog, ecological toxicity, and human health impacts) it may result in an oversimplification of the actual impacts because the indices are not tailored to localities. Also, during the interpretation step of the BIRDS LCAs, environmental impact results are optionally combined into a single environmental performance score using relative importance weights. These weights necessarily incorporate values and subjectivity. BIRDS users should routinely test the effects on the environmental impact scores of changes in the set of importance weights by completing their analysis with more than one weighting approach.

Life-cycle impact assessment is a rapidly evolving science. Assessment methods unheard of a decade ago have since been developed and are now being used routinely in LCAs. While BIRDS incorporates state-of-the-art impact assessment methods, the science will continue to evolve and methods in use today—-particularly those for land and water use — are likely to change and improve over time. Future versions of BIRDS should incorporate these improved methods as they become available.

Energy, environmental, and economic performance are but three attributes of building performance. The BIRDS model for the commercial database assumes that its building prototypes all meet minimum technical performance requirements. However, there may be significant differences in technical performance not evaluated in BIRDS for the commercial database, such as acoustic, fire, or indoor environmental quality performance (e.g. air quality, comfort, health), which may affect energy, environmental, and economic considerations.

The BIRDS sustainability results do not apply to buildings constructed in other countries where industry practices, fuel mixes, environmental regulations, transportation distances, and labor and material markets may differ. Furthermore, all buildings of a given type are not created equal. Building designs, sizes, useful lives, materials compositions, and costs will all vary for an individual building. The BIRDS results for a building prototype do not necessarily represent the performance of an individual building of that type. Future BIRDS-related tools should permit flexibility in building design and use of materials. 


\section{References}

Adomatis, S. (2015). What is Green Worth?, Adomatis Appraisal Services.

AHRI (2008). ANSI/AHRI 210/240-2008: 2008 Standard for Performance Rating of Unitary AirConditioning \& Air-Source Heat Pump Equipment.

ANSI/ASHRAE (2004). ANSI/ASHRAE Standard 62.1-2004, Ventilation for Acceptable Indoor Air Quality. Atlanta, GA, American Society of Heating, Refrigerating and Air-Conditioning Engineers.

Aroul, R. and J. A. Hansz (2012). "The Value of" Green:" Evidence from the First Mandatory Residential Green Building Program." Journal of Real Estate Research 34(1): 27-49.

ASHRAE (Undated). Equipment Life Expectancy Chart. http://culluminc.com/images/ASHRAE Chart HVAC Life Expectancy\%201.pdf., American Society of Heating, Refrigerating and Air-Conditioning Engineers (ASHRAE).

ASHRAE/AIA/IESNA (2009). Advanced Energy Design Guide for Highway Lodging. Atlanta, GA, American Society of Heating, Refrigerating and Air-Conditioning Engineers.

ASHRAE/IESNA Standard Project Committee 90.1 (1999). ASHRAE 90.1-1999 Standard - Energy Standard for Buildings Except Low-Rise Residential Buildings, ASHRAE, Inc.

ASHRAE/IESNA Standard Project Committee 90.1 (2004). ASHRAE 90.1-2004 Standard - Energy Standard for Buildings Except Low-Rise Residential Buildings, ASHRAE, Inc.

ASHRAE/IESNA Standard Project Committee 90.1 (2007). ASHRAE 90.1-2007 Standard - Energy Standard for Buildings Except Low-Rise Residential Buildings, ASHRAE, Inc.

ASHRAE/IESNA Standard Project Committee 90.1 (2010). ASHRAE 90.1-2010 Standard - Energy Standard for Buildings Except Low-Rise Residential Buildings, ASHRAE, Inc.

ASHRAE/IESNA Standard Project Committee 90.1 (2013). ASHRAE 90.1-2013 Standard - Energy Standard for Buildings Except Low-Rise Residential Buildings, ASHRAE, Inc.

ASHRAE/IESNA Standard Project Committee 90.1 (2016). ASHRAE 90.1-2016 Standard - Energy Standard for Buildings Except Low-Rise Residential Buildings, ASHRAE, Inc. 
ASTM (2011). Standard Practice for Applying the Analytic Hiearchy Process Multiattribute Decision Analysis of Investments Related to Buildings and Building Systems, ASTM Designation E1765-11. West Conshohocken, PA.

ASTM (2015). Standard Guide for Selecting Economic Methods for Evaluating Investments in Buildings and Building Systems, ASTM Designation E1185-15. West Conshohocken, PA.

ASTM (2015). Standard Practice for Measuring Life-Cycle Costs of Buildings and Building Systems, ASTM Designation E917-15. West Conshohocken, PA.

Athena Sustainable Materials Institute (2003). Exploratory Life-cycle Analysis of Residential Operating Energy Systems.

Atlas Roofing Corporation (2012). Energy Shield/Energy Shield Plus/Cavity Wall Material Safety Data Sheet.

Bae, Y. J. (2016). Integrated design tool of building system optimization for building life cycle cost, Purdue University.

Bare, J. (2011). "TRACI 2.0: the tool for the reduction and assessment of chemical and other environmental impacts 2.0." Clean Technologies and Environmental Policy 13(5): 687-696.

Bare, J., et al. (2006). "Development of the method and US normalization database for life cycle impact assessment and sustainability metrics." Environmental science \& technology 40(16): 5108-5115.

Bayer MaterialScience (2008). Energy and Environmental Benefits of Insulating Commercial Buildings with Polyiso.

Bayer MaterialScience (2011). Life-Cycle Assessment of Polyiso Insulation - Final Report, Bayer MaterialSchienve.

Belzer, D., et al. (2005). Analysis of Potential Benefits and Costs of Adopting a Commercial Building Energy Standard in South Dakota. Pacific Northwest National Laboratory (PNNL). Richland, WA.

Biswas, W. and M. Rosano (2011). "A life cycle greenhouse gas assessment of remanufactured refrigeration and air conditioning compressors." International Journal of Sustainable Manufacturing 2(23): 222-236.

Brundtland Commission (1987). Our Common Future: Report of the World Commission on Environment and Development. UN Documents Gatheringa Body of Global Agreements. 
Bureau of Labor Statistics (2013). "Green Goods and Services." Retrieved April 2017, 2017, from https://www.bls.gov/ggs/.

CBRE (2016). Whitesonte Research CostLab Tool.

Churcher, D. (2014). Life Cycle Assessment: An assessment of building services systems.

Cohen, S. (2006). "Green Buildings." The Washington Post. from http://www.washingtonpost.com/wpdyn/content/article/2006/08/03/AR2006080301410.html.

Daikin (2017). Vertical Stack Water Source Heat Pumps, Models WVHF Cabinet, WVHC Chassis (Standard Range), WVHW Chassis (Geothermal Range). Daikin.

Dastrup, S., et al. (2012). "Understanding the solar home price premium: electricity generation and "green" social status." European Economic Review 56(5): 961-973.

De Kleine, R. (2009). Life-cycle Optimization of Residential Air Conditioner Replacement, Report No. CSS09-12, University of Michigan, Ann Arbor.

Deru, M., et al. (2011). Commercial Reference Building Models of the National Building Stock. U.S. Department of Energy National Renewable Energy Laboratory.

Ecoinvent (2007). "Life-Cycle Inventory Database." from http://www.ecoinvent.org/database/.

Ecoinvent (2018). "ecoinvent 3 US-EI Life-Cycle Inventory Database." from http://www.ecoinvent.org/database/.

Eichholtz, P., et al. (2010). "Doing well by doing good? Green office buildings." The American Economic Review: 2492-2509.

Eichholtz, P., et al. (2013). "The economics of green building." Review of Economics and Statistics 95(1): 50-63.

Energy Efficiency and Renewable Energy (EERE) (2012). Life-Cycle Assessment of Energy and Environmental Impacts of LED Lighting Products Part 1: Review of the Life-Cycle Energy Consumption of Incandescent , Compact Fluorescent, and LED Lamps, U.S. Department of Energy.

EnergyPlus (n.d.). "Weather Data by Region." 2016, from https://energyplus.net/weatherregion/north and central america wmo region 4/USA. 
Fuller, S. and S. Petersen (1996). "Life-Cycle Costing Manual for the Federal Energy Management Program, 1995 Edition." NIST handbook 135.

Gloria, T. P., et al. (2007). "Life-Cycle Impact Assessment Weights to Support Environmentally Preferable Purchasing in the United States." Environmental science \& technology 41(21): 7551-7557.

Goedkoop, M., et al. (2008). "A Life-Cycle Impact Assessment Method which Comprises Harmonised Category Indicators at the Midpoint and the Endpoint Level. Report I: Characterisation." from www.Iciarecipe.net.

Green America (2013). "The big green opportunity for small business in the US: Small business sustainability report." Retrieved August 2017, 2017, from http://biggreenopportunity. org/wpcontent/uploads/2013/05/Big-Green-Opportunity-Report-FINAL-WEB. pdf

Greening, B. a. A., A. (2012). "Domestic heat pumps: Life cycle environmental impacts and potential implications for the UK." Energy 39: 205 - 217.

Griffin, A., et al. (2009). "Certified home performance: assessing the market impacts of third party certification on residential properties." Portland, OR: Earth Advantage Institute.

Guinée, J. B. (2002). "Handbook on Life-Cycle Assessment Operational Guide to the ISO Standards." The International Journal of Life Cycle Assessment 7(5): 311-313.

Hendrickson, C., et al. (2006). Environmental Life-Cycle Assessment of Goods and Services: An InputOutput Approach, Resources for the Future Press.

Hoen, B. (2011). "An analysis of the effects of residential photovoltaic energy systems on home sales prices in California." Lawrence Berkeley National Laboratory.

Intergovernmental Panel on Climate Change (IPCC) (2005). IPCC/TEAP Special Report: Safeguarding the Ozone Layer and the Global Climate System.

International Copper Association (ICA) (2012). Life-cycle Assessment of Primary Copper Cathode.

International Organization for Standardization (ISO) (2006a). Environmental Management -- Life-Cycle Assessment -- Principles and Framework, International Standard 14040.

International Organization for Standardization (ISO) (2006b). Environmental Management -- Life-Cycle Assessment -- Principles and Framework, International Standard 14044. 
IronHorse (2017). IronHorse Rolled-Steel AC Motors- 1-Phase 56C/56HC Frame TEFC Motors. https://cdn.automationdirect.com/static/specs/ironhorsesprs.pdf.

Johnson Controls (2015). Johnson Controls York Engineering Guide: MODEL VDCF DIRECT DRIVE REMOTE AIR-COOLED FLUID COOLERS. https://www.johnsoncontrols.com/-/media/ici/be/united-states/hvacequipment/condensoers-and-condensing-units/files/be engguide vdcfaircooledfluidcooler-(1).pdf.

Kahn, M. E. and N. Kok (2014). "The capitalization of green labels in the California housing market." Regional Science and Urban Economics 47: 25-34.

Kneifel, J. (2012b ). "Prototype Commercial Buildings for Energy and Sustainability Assessment: Design Specification, Life-Cycle Costing and Carbon Assessment." NIST Technical Note 1765.

Kok, N. and M. Kahn (2012). The Value of Green Labels in the California Housing Market: An Economic Analysis of the Impact of Green Labeling on the Sales Price of a Home. Berkely, CA, University of California, Berkely.

Lavappa, P. and J. Kneifel (2016). Energy Price Indices and Discount Factors for Life-Cycle Cost Analysis 2016, Annual Supplement to NIST Handbook 135. National Institute of Standards and Technology.

Levin, L. (1996). Best Sustainable Indoor Air Quality Practices in Commercial Buildings. Third International Green Building Conference and Expostion - 1996, Gaithersburg, MD, NIST.

Lippiatt, B., et al. (2013). "Building Industry Reporting and Design for Sustainability (BIRDS) Technical Manual and User Guide." NIST Technical Note 1814.

Lufkin, P., et al. (2010). The Whitestone Facility Maintenance and Repair Cost Reference 2010-2011, Whitestone Research Corporation.

McGraw-Hill Construction (2017). World Green Building Trends 2016: United States. Smart Market Report, Dodge Data and Analytics

Meakin, S. (1992). The Rio Earth Summit: Summary of the United Nations Conference on Environment and Development, Library of Parliament, Research Branch.

National Association of Home Builders (NAHB) Research Center (2007). Study of Life Expectancy of Home Components, National Association of Home Builders. 
National Institute of Building Sciences (2017). "Green Building Standards and Certification Systems." Retrieved April 2017, 2017, from https://www.wbdg.org/resources/green-building-standards-andcertification-systems.

National Renewable Energy Laboratory (NREL) (2012). "U.S. Life-Cycle Inventory Database (LCI)." from http://www.nrel.gov/lci/.

NIST (2010). Building for Environmental and Economic Sustainability (BEES) Online Software, Applied Economics Office, Engineering Laboratory

NREL (2015). "U.S. Life-Cycle Inventory Database (LCI)." from http://www.nrel.gov/Ici/.

Nutone (2017). Nutone Technical Specifications: Q7RF Series Two Stage Packaged Heat Pump, Single Phase. http://literature.nutonehvac.com/NuTone/HVAC/Heat\%20Pump\%20\%20Packaged/Q7RF/Technical\%20Specifications/16 SEER Two Stage Packaged Heat Pump 851e.pdf.

Nyman, M. a. C. J. S. (2005). "Life cycle assessment of residential ventilation units in a cold climate. ." Building and Environment 40: 15-27.

OSRAM Opto Semiconductors GmbH. (2009). Life-cycle Assessment of Illuminants - A Comparison of Light Bulbs, Compact Fluorescent Lamps and LED Lamps (Executive Summary).

Parsons, D. (2006). "The environmental impact of compact fluorescent lamps and incandescent lamps for Australian conditions." The Environmental Engineer 7(2): 8-14.

PE International (2010). Eco-Profile of Aromatic Polyester Polyols (APP).

PE International (2011). GaBi Database.

Pfleger, W., et al. (2011). "Market impacts of Energy Star qualification for new homes." North Carolina Energy Efficiency Alliance.

Ramroth, L. (2008). Comparison of Life-Cycle Analyses of Compact Fluorescent and Incandescent Lamps Based on Rated Life of Compact Fluorescent Lam, Rocky Mountain Institute (RMI).

Ramroth, L. (2008). "Comparison of life-cycle analyses of compact fluorescent and incandescent lamps based on rated life of compact fluorescent lamp." Rocky Mountain Institute: 11-12. 
Rosenbaum, R. K., et al. (2011). "USEtox human exposure and toxicity factors for comparative assessment of toxic emissions in life cycle analysis: sensitivity to key chemical properties." The International Journal of Life Cycle Assessment 16(8): 710-727.

Siegel, J. J. (2014). "Earnings, Inflation, and Future Stock and Bond Returns 1." Proceedings of the American Philosophical Society 158(3): 222.

Suh, S. (2005). "Developing a sectoral environmental database for input-output analysis: the comprehensive environmental data archive of the US." Economic Systems Research 17(4): 449-469.

Suh, S. (2010). Comprehensive Environmental Data Archive (CEDA). The Sustainability Practictioner's Guide to Input-Output Analysis. J. Murray and R. Wood, Common Ground Publishing.

Suh, S. and G. Huppes (2005). "Methods for life cycle inventory of a product." Journal of Cleaner Production 13(7): 687-697.

Suh, S., et al. (2004). "System boundary selection in life-cycle inventories using hybrid approaches." Environmental Science \& Technology 38(3): 657-664.

Suh, S. and B. C. Lippiatt (2012). "Framework for hybrid life cycle inventory databases: a case study on the Building for Environmental and Economic Sustainability (BEES) database." The International Journal of Life Cycle Assessment 17(5): 604-612.

Tähkämö, L. e. a. (2013). "Life cycle assessment of fluorescent lamp luminaire used in industry premises - a case study." Lighting Res. Technol. 0: 1-12.

Thornton, B. A., et al. (2011). Achieving the $30 \%$ goal: Energy and cost savings analysis of ASHRAE Standard 90.1-2010, Pacific Northwest National Laboratory (PNNL), Richland, WA (US).

Trane (2000). Trane Product Specifications - Trane CAB-PRC001-EN. http://www.trane.com/download/equipmentpdfs/cabprc001en.pdf.

Trane (2014). Trane Product Catalog Indoor Gas Heating Products: Unit Heaters, Duct Furnaces. Trane Unit Heater Catalog.

U.K. Department for Environment, Food and Rural Affairs (Defra) (2009). Life-cycle Assessment of UltraEfficient Lamps.

U.S. Congress (2007). H.R. 6: Energy Independence and Security Act (EISA) of 2007. P.L. 110-140. 
U.S. EPA Science Advisory Board (1990). Reducing Risk: Setting Priorities and Strategies for Environmental Protection, SAB-EC-90-021. Washington, DC.

U.S. EPA Science Advisory Board (2000). Toward Integrated Environmental Decision-Making, EPA-SABEC-00-011. Washington, DC.

U.S. Green Building Council (2015). 2015 Green Building Economic Impact Study, USGBC.

U.S. Green Building Council (2017). "LEED Projects and People Databases." Retrieved April 2017, 2017, from https://www.usgbc.org/.

United Nations Environment Programme (UNEP) (2005). Report of the Technology and Economic Assessment Panel (TEAP).

US Department of Energy (DOE) (n.d.). "Commercial Prototype Building Models." Retrieved 08/14/2017, 2017, from https://www.energycodes.gov/development/commercial/prototype models.

World Steel Association (2011). Methodology report - Life-cycle Inventory Study for Steel Products.

Yang, L., et al. (2008). "Comparison of environmental impacts of two residential heating systems." Building and Environment 43(6): 1072-1081. 